Prepared in cooperation with Colorado River Water Conservation District, Eagle County, Eagle River Water and Sanitation District, Upper Eagle Regional Water Authority, Colorado Department of Transportation, City of Aurora, Town of Eagle, Town of Gypsum, Town of Minturn, Town of Vail, Vail Resorts, City of Colorado Springs, Colorado Springs Utilities, and Denver Water

\title{
Assessment of Surface-Water Quantity and Quality, Eagle River Watershed, Colorado, 1947-2007
}
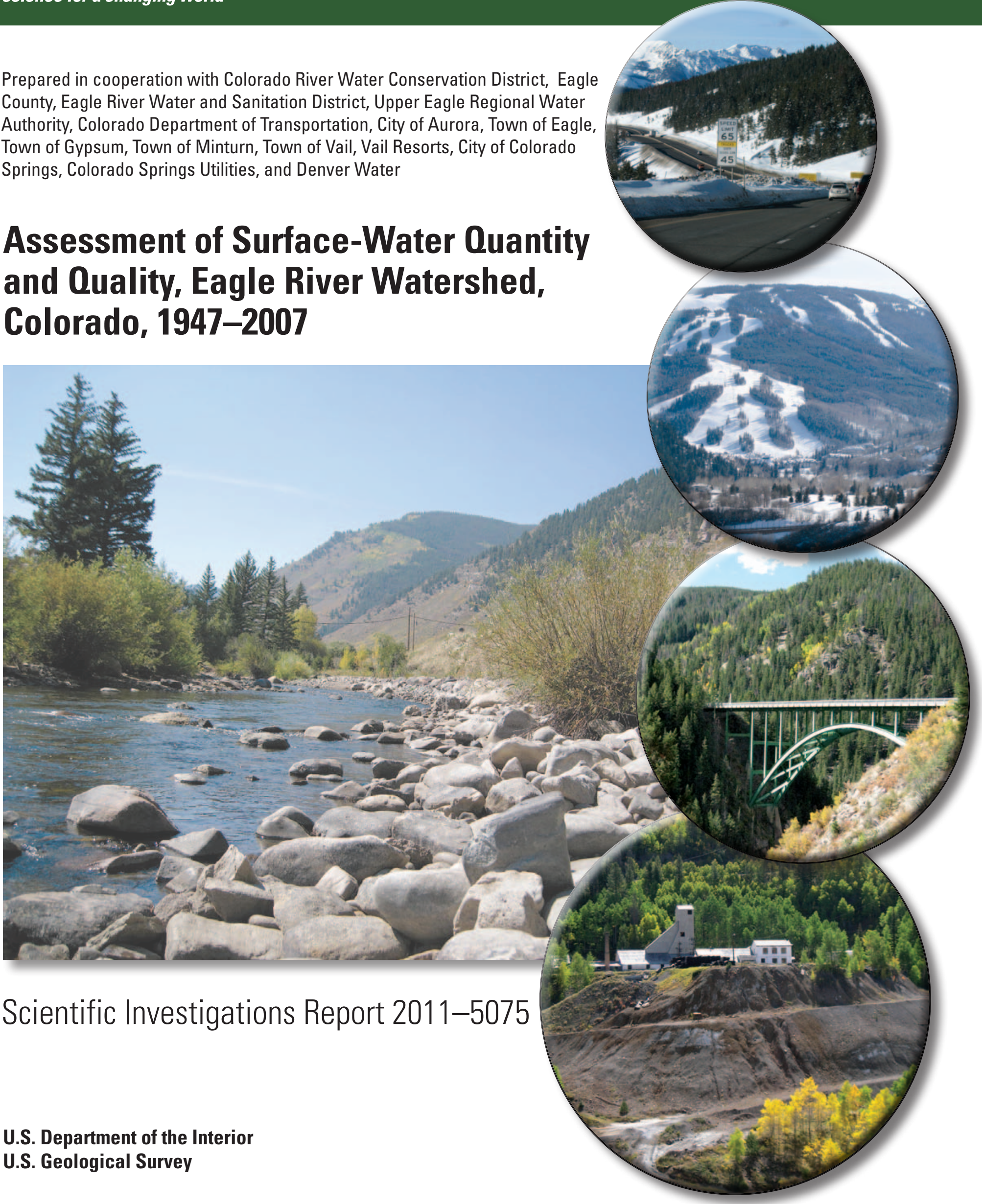


\section{Cover.}

Large photo: Eagle River at the mouth near Minturn, Colorado. Photograph by Jennifer Moore, September 15, 2010.

Small photos from top to bottom: Vail Pass, Vail, Colorado. Photograph by Jennifer Moore, February 1, 2009.

Beaver Creek Ski Resort, Beaver Creek, Colorado. Photograph by Jennifer Moore, February 1, 2009.

Bridge near Red Cliff, Colorado. Photograph by Jennifer Moore, September 14, 2010.

Old mining area near Gilman, Colorado. Photograph by Jennifer Moore, September 14, 2010. 


\section{Assessment of Surface-Water Quantity and Quality, Eagle River Watershed, Colorado, 1947-2007}

By Cory A. Williams, Jennifer L. Moore, and Rodney J. Richards

Prepared in cooperation with Colorado River Water Conservation District, Eagle County, Eagle River Water and Sanitation District, Upper Eagle Regional Water Authority, Colorado Department of Transportation, City of Aurora, Town of Eagle, Town of Gypsum, Town of Minturn, Town of Vail, Vail Resorts, City of Colorado Springs, Colorado Springs Utilities, and Denver Water

Scientific Investigations Report 2011-5075 


\section{U.S. Department of the Interior \\ KEN SALAZAR, Secretary \\ U.S. Geological Survey \\ Marcia K. McNutt, Director}

\section{U.S. Geological Survey, Reston, Virginia: 2011}

For more information on the USGS - the Federal source for science about the Earth, its natural and living resources, natural hazards, and the environment: visit http://www.usgs.gov or call 1-888-ASK-USGS.

For an overview of USGS information products, including maps, imagery, and publications, visit http:// www.usgs.gov/pubprod

To order this and other USGS information products, visit http://store.usgs.gov

Any use of trade, product, or firm names is for descriptive purposes only and does not imply endorsement by the U.S. Government.

Although this report is in the public domain, permission must be secured from the individual copyright owners to reproduce any copyrighted material contained within this report.

Suggested citation:

Williams, C.A., Moore, J.L., and Richards, R.J., 2011, Assessment of surface-water quantity and quality, Eagle River watershed, Colorado, 1947-2007: U.S. Geological Survey, Scientific Investigation Report 2011-5075, 139 p. 


\section{Contents}

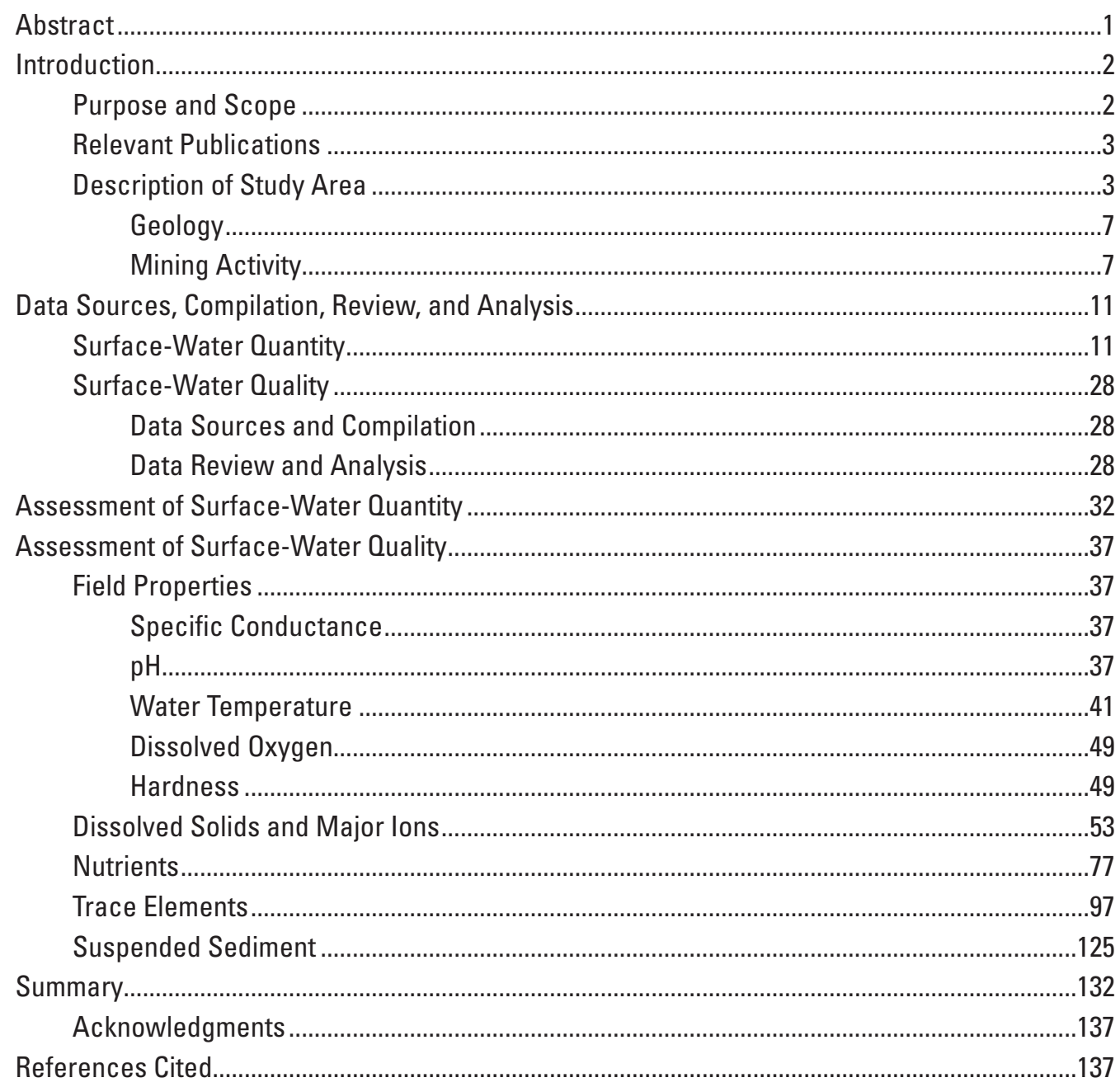

\section{Figures}

1-12. Map showing:

1. Location of the Eagle River watershed, Colorado, and location of major streams, tributaries, and urban development within the watershed

2. Variation in precipitation throughout the Eagle River watershed, Colorado (Western Regional Climate Center, 2009) ....................................................................

3. Land use in the Eagle River watershed, Colorado, 2008..........................................6

4. Surficial geology of the Eagle River watershed, Colorado (from Tweto, 1979)..........9

5. Location of hard-rock mining and related superfund remediation in the Eagle River watershed, Colorado, 2008

6. Streamflow-gaging station locations and streamflow distribution for selected streams in the Eagle River watershed, Colorado, by subwatershed. 
7. Locations of surface-water-monitoring sites within the Eagle River watershed, Colorado, 1947-2007.

8. Locations of surface-water-monitoring sites in the lower Eagle River subwatsershed, Colorado, 1947-2007

9. Location of surface-water-monitoring sites in the middle Eagle River subwatershed, Colorado, 1947-2007.

10. Location of surface-water-monitoring sites in the Upper Eagle River subwatershed, Colorado, 1947-2007 26

11. Location of surface-water-monitoring sites in the Gore Creek subwatershed, Colorado, 1947-2007

12. Colorado Department of Public Health and Environment stream-segment designations for the Eagle River watershed, Colorado (Colorado Department of Public Health and Environment and U.S. Environmental Protection Agency, 2008)

13. Hydrographs of selected sites in the Eagle River watershed, Colorado. . .33

14. Graph showing lowess Smooth of annual-mean streamflow at selected sites in the Eagle River watershed, Colorado, 1947-2007

15. Arrow schematic of major streams and diversion mean annual streamflow volume in the Eagle River watershed

16. Graph showing comparison of streamflow prediversion (1945-1965) and postdiversion (1966-2008) from Homestake Reservoir at site 27, Homestake Creek near Red Cliff, Colorado

17. Map showing spatial distribution of median-concentration percentiles and monotonic trends in specific conductance at selected sites in the Eagle River watershed, Colorado, 1990-2007

18. Graph showing seasonal pattern of $\mathrm{pH}$ at selected sites in the Eagle River watershed, Colorado, 1947-2007

19-21. Map showing:

19. Spatial distribution of median-concentration percentiles and monotonic $\mathrm{pH}$ trends at selected sites in the Eagle River watershed, Colorado, 1990-2007.

20. Number of exceedances of the Colorado water-quality standard for $\mathrm{pH}$ at selected sites in the Eagle River watershed, Colorado, 1947-2007.

21. Spatial distribution of median-concentration percentiles and monotonic water temperature trends at selected sites in the Eagle River watershed, Colorado, 1990-2007.

22. Graph showing seasonal pattern of water temperature at selected sites in the Eagle River watershed, Colorado, 1947-2007

23. Map showing number of exceedances of the Colorado water-quality standard for water temperature at selected sites in the Eagle River watershed, Colorado, 1947-2007.

24. Map showing spatial distribution of median-concentration percentiles and monotonic dissolved-oxygen concentration trends at selected sites in the Eagle River watershed, Colorado, 1990-2007

25. Graph showing seasonal pattern of dissolved oxygen at selected sites in the Eagle River watershed, Colorado, 1947-2007

26. Map showing number of exceedances of the Colorado water-quality standard for dissolved oxygen at selected sites in the Eagle River watershed, Colorado, 1947-2007. 
27. Map showing spatial distribution of median-concentration percentiles and monotonic hardness concentration trends at selected sites in the Eagle River watershed, Colorado, 1994-2007

28. Graph showing seasonal pattern of hardness concentrations at selected sites in the Eagle River watershed, Colorado, 1947-2007

29. Map showing spatial distribution of median-concentration percentiles and monotonic sum of constituent trends at selected sites in the Eagle River watershed, Colorado, 1995-2007.

30-37. Graphs showing:

30. Seasonal pattern of sum of constituents at selected sites in the Eagle River watershed, Colorado, 1947-2007.

31. Seasonality of water chemistry at site 13, Eagle River watershed, Colorado, 1947-2007

32. Seasonality of water chemistry at site 160 , Eagle River watershed, Colorado, 1947-2007

33. Seasonality of water chemistry at site 173 , Eagle River watershed, Colorado, 1947-2007.

34. Seasonality of water chemistry at site 201, Eagle River watershed, Colorado, 1947-2007.

35. Seasonality of water chemistry at site 224, Eagle River watershed, Colorado, 1947-2007.

36. Seasonality of water chemistry at selected streamflow-gaging station locations, in the Eagle River watershed, Colorado, 1947-2007.

37. Spatial distribution of median-concentration percentiles and monotonic calcium concentration trends at selected sites in the Eagle River watershed, Colorado, 1995-2007

38-50. Map showing:

38. Spatial distribution of median-concentration percentiles and monotonic magnesium trends at selected sites in the Eagle River watershed, Colorado, 1995-2007.

39. Spatial distribution of median-concentration percentiles and monotonic sodium trends at selected sites in the Eagle River watershed, Colorado, 1995-2007.

40. Spatial distribution of median-concentration percentiles and monotonic potassium trends at selected sites in the Eagle River watershed, Colorado, 1995-2007.

41. Spatial distribution of median-concentration percentiles and monotonic chloride trends at selected sites in the Eagle River watershed, Colorado, 1995-2007.

42. Spatial distribution of median-concentration percentiles and monotonic sulfate trends at selected sites in the Eagle River watershed, Colorado, 1995-2007.

43. Spatial distribution of median-concentration percentiles and monotonic silica trends in at selected sites in the Eagle River watershed, Colorado, 1995-2007.

44. Spatial distribution of median-concentration percentiles and monotonic alkalinity trends at selected sites in the Eagle River watershed, Colorado, 1995-2007. 
45. Number of exceedances of the Colorado water-quality standard for major ions at selected sites in the Eagle River watershed, Colorado, 1947-2007

46. Spatial distribution of median-concentration percentiles and monotonic nitrate trends at selected sites in the Eagle River watershed, Colorado, 1995-2007.

47. Spatial distribution of median-concentration percentiles and monotonic nitrite trends at selected sites in the Eagle River watershed, Colorado, 1995-2007 .........81

48. Spatial distribution of median-concentration percentiles and monotonic ammonia trends at selected sites in the Eagle River watershed, Colorado, 1995-2007 .........82

49. Spatial distribution of median-concentration percentiles and monotonic total phosphorus trends at selected sites in the Eagle River watershed, Colorado, 1995-2007.

50. Spatial distribution of median-concentration percentiles and monotonic orthophosphate trends at selected sites in the Eagle River watershed, Colorado, 1995-2007

51-60. Graphs showing:

51. Seasonal patterns of nitrate concentrations at selected sites in the Eagle River watershed, Colorado, 1947-2007

52. Seasonal patterns of nitrite concentrations at selected sites in the Eagle River watershed, Colorado, 1947-2007.

53. Seasonal patterns of total phosphorus concentrations at selected sites in the Eagle River watershed, Colorado, 1947-2007.

54. Seasonal patterns of orthophosphate concentrations at selected sites in the Eagle River watershed, Colorado, 1947-2007

55. Temporal distribution of total phosphorus concentrations at site 110, Eagle River watershed, Colorado, 1997-2004.

56. Temporal distribution of total phosphorus concentrations at site 157, Eagle River watershed, Colorado, 1974-2006.

57. Temporal distribution of total phosphorus concentrations at site 160, Eagle River watershed, Colorado, 1996-2007.

58. Temporal distribution of total phosphorus concentrations at site 180, Eagle River watershed, Colorado, 1970-2002.

59. Temporal distribution of total phosphorus concentrations at site 224, Eagle River watershed, Colorado, 1980-2007.

60. Temporal distribution of total phosphorus concentrations at site 227, Eagle River watershed, Colorado, 1974-2007.

61. Map showing number of exceedances of the Colorado chronic water-quality standard for nutrients at selected sites in the Eagle River watershed, Colorado, 1970-2007

62. Map showing number of exceedances of the Colorado acute water-quality standard for nutrients at selected sites in the Eagle River watershed, Colorado, 1947-2007.

63. Graph showing correlation between suspended-sediment concentration and total phosphorus concentration in the Eagle River watershed, Colorado.

64-68. Map showing:

64. Spatial distribution of median-concentration percentiles and monotonic dissolved cadmium trends at selected sites in the Eagle River watershed, Colorado, 1994-2006.

65. Spatial distribution of median-concentration percentiles for dissolved copper at selected sites in the Eagle River watershed, Colorado, 1966-2007......102 
66. Spatial distribution of median-concentration percentiles and monotonic dissolved iron trends at selected sites in the Eagle River watershed,

Colorado, 1994-2006

67. Spatial distribution of median-concentration percentiles and monotonic dissolved manganese trends at selected sites in the Eagle River watershed, Colorado,1994-2006

68. Spatial distribution of median-concentration percentiles and monotonic dissolved zinc concentration trends at selected sites in the Eagle River watershed, Colorado, 1994-2006

69-75. Graphs showing:

69. Seasonal pattern of dissolved cadmium concentrations at selected sites in the Eagle River watershed, Colorado, 1966-2007

70. Seasonal pattern of dissolved copper concentrations at selected sites in the Eagle River watershed, Colorado, 1966-2007

71. Seasonal pattern of dissolved iron concentrations at selected sites in the Eagle River watershed, Colorado, 1966-2007

72. Seasonal pattern of dissolved manganese concentrations at selected sites in the Eagle River watershed, Colorado, 1970-2007

73. Seasonal patterns of dissolved zinc concentrations at selected sites in the Eagle River watershed, Colorado, 1966-2007 110

74. Seasonal pattern relation between trace elements concentrations and hardness at site 72 in the Eagle River watershed, Colorado

75. Seasonal patterns of total recoverable iron concentrations at site 224 in the Eagle River watershed, near Gypsum, Colorado

76. Map showing number of exceedances of the Colorado acute water-quality standard for concentration of trace elements (dissolved arsenic, dissolved selenium, dissolved uranium) at selected sites in the Eagle River watershed, Colorado, 1971-2007

77. Map showing number of exceedances of the Colorado chronic water-quality standard for concentration of trace elements (dissolved arsenic, dissolved selenium, dissolved uranium) at selected sites in the Eagle River watershed, Colorado, 1968-2001

78-96. Graphs showing:

78. Temporal distribution of dissolved cadmium concentrations at site 28 , in the Eagle River watershed, Colorado, 1984-2003

79. Temporal distribution of dissolved cadmium concentrations at site 66, in the Eagle River watershed, Colorado, 1985-2007

80. Temporal distribution of dissolved cadmium concentrations at site 173, in the Eagle River watershed, 1997-2007

81. Temporal distribution of dissolved copper concentrations at site 28, in the Eagle River watershed, Colorado, 1985-2003

82. Temporal distribution of dissolved copper concentrations at site 66, in the Eagle River watershed, Colorado, 1981-2007

83. Temporal distribution of dissolved copper concentrations at site 173, in the Eagle River watershed, Colorado, 1994-2007

84. Temporal distribution of dissolved iron concentrations at site 28, in the Eagle River watershed, Colorado, 1984-2003

85. Temporal distribution of dissolved iron concentrations at site 66 , in the Eagle River watershed, Colorado, 1985-2005 
86. Temporal distribution of dissolved iron concentrations at site 173 ,

in the Eagle River watershed, Colorado, 1978-2003.

87. Temporal distribution of total recoverable iron concentrations at site 28 , in the Eagle River watershed, Colorado, 1984-2004.

88. Temporal distribution of total recoverable iron concentrations at site 66, in the Eagle River watershed, Colorado, 1985-2007.

89. Temporal distribution of total recoverable iron concentrations at site 173, in the Eagle River watershed, Colorado, 1994-2006.

90. Temporal distribution of dissolved manganese concentrations at site 28, in the Eagle River watershed, Colorado, 1984-2003.

91. Temporal distribution of dissolved manganese concentrations at site 66, in the Eagle River watershed, Colorado, 1986-2007.

92. Temporal distribution of dissolved manganese concentrations at site 173, in the Eagle River watershed, Colorado, 1994-2007.

93. Temporal distribution of dissolved zinc concentrations at site 23 , in the Eagle River watershed, Colorado, 1985-2003.

94. Temporal distribution of dissolved zinc concentrations at site 66 , in the Eagle River watershed, Colorado, March-April 1986-2007

95. Temporal distribution of dissolved zinc concentrations at site 66, in the Eagle River watershed, Colorado, May-February 1986-2007

96. Temporal distribution of dissolved zinc concentrations at site 173 , in the Eagle River watershed, 1994-2006

97-99. Maps showing:

97. Number of exceedances of the Colorado acute water-quality standard for metals concentrations at selected sites in the Eagle River watershed, Colorado, 1966-2006

98. Number of exceedances of the Colorado chronic water-quality standard for metals concentrations at selected sites in the Eagle River watershed, Colorado, 1966-2007

99. Spatial distribution of median-concentration percentiles and monotonic suspended-sediment concentration trends at selected sites in the Eagle River watershed, Colorado, 1995-2007

100. Graph showing seasonal pattern in suspended-sediment concentration at selected sites in the Eagle River watershed, Colorado, 1947-2007.

\section{Tables}

1. Sources of water-related data for the Eagle River watershed

2. Eagle Mine Superfund Site major construction milestones (Colorado Department of Public Health and Environment and U.S. Environmental Protection Agency, 2008).....

3. Hydrologic characteristics for streamflow-gaging stations in the Eagle River watershed.

4. Summary of the mean annual runoff, median annual maximum daily streamflow, annual flood frequency, and low-flow statistics for selected sites in the Eagle River watershed.

5. Historic and current surface-water quantity and quality sites in the Eagle River watershed, Colorado.

6. Eagle River watershed quality-assurance results by sample source agency 
7. Federal Clean Water Act Section 303(d) listings for the Eagle River watershed .30

8. Summary statistics of field properties and suspended-sediment data in the Eagle River watershed by subwatershed, 1947-2007.

9. Summary of monotonic temporal trends for field properties and suspended-sediment concentrations in the Eagle River watershed, Colorado.

10. Summary of Colorado exceedances of water-quality standards for field properties in the Eagle River watershed, Colorado by subwatershed.

11. Summary statistics of dissolved solids and major ions data in the Eagle River watershed, Colorado by subwatershed 1947-2007

12. Summary of monotonic temporal trends for dissolved solids and major ions in the Eagle River watershed, Colorado.

13. Summary of exceedences of Colorado water-quality standard ( 250 milligrams per liter) for chloride for surface-water samples collected in the Eagle River watershed, 1947-2007

14. Comparison of cation and anion concentration, Gore Creek subwatershed, Colorado, 1947-2007.

15. Median nutrient concentrations for selected sites in the Eagle River watershed, Colorado, 1968-2007 and background regional reference values .

16. Summary statistics of nutrients data in the Eagle River watershed, Colorado, by subwatershed

17. Summary of water-quality standard or recommendation exceedances for nutrients in the Eagle River watershed by subwatershed

18. Summary of monotonic temporal trends for nutrients in the Eagle River watershed.....93

19. Summary statistics of trace element data in the Eagle River watershed by subwatershed 1947-2007

20. Summary of water-quality standard exceedances for trace elements in the Eagle River watershed by subwatershed. .115

21. Summary of step-trends assessment for select sites affected by Eagle Mine Superfund remediation, water years 1972-1991, and 1992-2007

22. Summary of monotonic temporal trends for metals in the Eagle River watershed.......131 



\title{
Assessment of Surface-Water Quantity and Quality, Eagle River Watershed, Colorado, 1947-2007
}

\author{
By Cory A. Williams, Jennifer L. Moore, and Rodney J. Richards
}

\section{Abstract}

From the early mining days to the current tourismbased economy, the Eagle River watershed (ERW) in central Colorado has undergone a sequence of land-use changes that has affected the hydrology, habitat, and water quality of the area. In 2000, the USGS, in cooperation with the Colorado River Water Conservation District, Eagle County, Eagle River Water and Sanitation District, Upper Eagle Regional Water Authority, Colorado Department of Transportation, City of Aurora, Town of Eagle, Town of Gypsum, Town of Minturn, Town of Vail, Vail Resorts, City of Colorado Springs, Colorado Springs Utilities, and Denver Water, initiated a retrospective analysis of surface-water quantity and quality in the ERW.

Surface-water quantity data and surface-water quality data were obtained from local, State, and Federal agencies to assist in the analysis of surface-water conditions in the ERW 1947-

2007. Surface-water-quality data from 293 sites and 12 different source agencies were compiled into 192 unique sites located on streams and rivers in the ERW. Approximately 39 percent of the unique sites had fewer than 5 samples; while 23 percent of the sites had more than 100 samples. Physical properties were the most abundant type of samples collected, with major ions, nutrients, and trace elements also commonly collected.

For selected water-quality properties and constituents in the watershed, this report: (1) characterizes available waterquantity and water-quality data, (2) identifies spatial and seasonal variability in water quantity and water quality, (3) provides comparisons to Federal and State water-quality standards or recommendations, (4) characterizes temporal changes in water quality, and (5) where possible, identifies potential causes of these changes. This report provides reconnaissancelevel statistical summaries and comparisons of water-quality conditions and characteristics using available data within the ERW. The report also includes streamflow statistics such as: mean annual runoff totals, peak-flood-frequency recurrence intervals, and minimum 7-day mean streamflows for selected sites within the watershed.

The spatial patterns for concentrations of trace metals (aluminum, cadmium, copper, iron, manganese, and zinc) indicate an increase in dissolved concentrations of these metals near historical mining areas in the Eagle River and several tributaries near Belden. In general, concentrations decrease downstream from mining areas. Concentrations typically are near or below reporting limits in Gore Creek and other tributaries within the watershed. Concentrations for trace elements (arsenic, selenium, and uranium) in the watershed usually are below the reporting limit, and no prevailing spatial patterns were observed in the data. Steptrend analysis and temporal-trend analysis provide evidence that remediation of historical mining areas in the upper Eagle River have led to observed decreases in metals concentrations in many surface-waters. Comparison of pre- and post-remediation concentrations for many metals indicates significant decreases in metals concentrations for cadmium, manganese, and zinc at sites downstream from the Eagle Mine Superfund Site. Some sites show order of magnitude reductions in median concentrations between these two periods. Evaluation of monotonic trends for dissolved metals concentrations show downward trends at numerous sites in, and downstream from, historic mining areas.

The spatial pattern of nutrients shows lower concentrations on many tributaries and on the Eagle River upstream from Red Cliff with increases in nutrients downstream of major urban areas. Seasonal variations show that for many nutrient species, concentrations tend to be lowest May-June and highest January-March. The gradual changes in concentrations between seasons may be related to dilution effects from increases and decreases in streamflow. Upward trends in nutrients between the towns of Gypsum and Avon were detected for nitrate, orthophosphate, and total phosphorus. An upward trend in nitrite was detected in Gore Creek. No trends were detected in un-ionized ammonia within the ERW. Exceedances of State water-quality standards (nitrite, nitrate, and un-ionized ammonia) and levels higher than U.S. Environmental Protection Agency recommendations (total phosphorus) occur in several areas within the ERW. The majority of the exceedances are from comparisons to the U.S. Environmental Protection Agency total phosphorus recommendations. A positive correlation was observed between suspended sediment and total phosphorus.

An upward trend in total dissolved solids in Gore Creek may be the result of increases in chloride salts. Highly significant trends were detected in sodium, potassium, and chloride with a significant upward trend in magnesium and 
a weakly significant upward trend in calcium. A quantitative analysis of the relative abundance of calcium, magnesium, sodium, and potassium to the available anions suggests that chloride salts likely are the source for the detected upward trends because chloride is the only commonly occurring anion with a trend in Gore Greek. A potential source for the observed chloride salts may be the chemical anti-icing and deicing products used during winter road maintenance in municipal areas and on Interstate-70.

A downward trend in dissolved solids in the Eagle River between Gypsum and Avon may be contributing to the detected trend on the Eagle River at Gypsum. Significant downward trends were detected in specific ions such as calcium, magnesium, sulfate, and silica. Measures of total dissolved solids as well as comparisons to specific ions show that in water-quality samples within the ERW concentrations generally are lower in the headwaters, with increases downstream from Wolcott. Differences in concentrations likely result from increased abundance of salt-bearing geologic units downstream from Avon. Few sites had measured concentrations that exceeded the State standards for chloride.

\section{Introduction}

From the early mining days to the current tourism-based economy, the Eagle River watershed (ERW) (fig. 1) in central Colorado has undergone a sequence of land-use changes that has affected the hydrology, habitat, and water quality of the area. Although intensive hard-rock mining has all but stopped in the ERW, elevated concentrations of metals, such as cadmium, copper, iron, manganese, and zinc, in streams are prevalent as a result of long-standing mining activity in the watershed (Colorado Department of Public Health and Environment, 2008). A 235-acre site encompassing the Eagle Mine and associated mining wastes between Gilman and Minturn was placed on the Superfund site list in 1986 (Colorado Department of Public Health and Environment, 2008). Remediation of the Eagle Mine site began in 1988 and continues today (2008). Eagle County has become a four-season resort destination. Ski resorts such as Vail and Beaver Creek have brought increased tourism and development to the area.

Although remediation of mining-affected areas continues, expansion of development, tourism, and transportation place new and varied demands on the water resources of the ERW. Local entities in the watershed that rely on and manage these water resources are interested in the assessment of water quantity and water quality to aid in the preservation and management of the ERW. Beginning in 1997, in cooperation with the Gore Creek Management Program partners, the U.S. Geological Survey (USGS) began a water-quality monitoring program on Gore Creek. The evaluation of this monitoring program was completed in 2001 and summarized in a USGS report (Wynn and others, 2001). The program was expanded in 1999 to include additional water-quantity and water-quality monitoring throughout the ERW. In order to evaluate the water quality, assess effects of growth and associated land-use change, identify temporal and spatial gaps within available water-quality data, and evaluate spatial and temporal trends in water quality, an analysis of historical data was done. In 2000, the USGS, in cooperation with the Colorado River Water Conservation District, Eagle County, Eagle River Water and Sanitation District, Upper Eagle Regional Water Authority, Colorado Department of Transportation, City of Aurora, Town of Eagle, Town of Gypsum, Town of Minturn, Town of Vail, Vail Resorts, City of Colorado Springs, Colorado Springs Utilities, and Denver Water, initiated a retrospective analysis of surface-water quantity and quality in the ERW.

\section{Purpose and Scope}

This report presents an assessment of the available surfacewater quantity and quality data from 1947 to 2007 for the ERW. Surface water in this study refers to rivers and streams; analysis of wells, groundwater, mine drainage, and ditch diversions were not included in this report. For selected water-quality properties and constituents in the watershed, this report: (1) characterizes available water-quantity and water-quality data, (2) identifies spatial and seasonal variability in water quantity and water quality, (3) provides comparisons to Federal and State water-quality standards or recommendations, (4) characterizes temporal changes in water quality, and (5) where possible, identifies potential causes of these changes.

The analysis done by the U.S. Geological Survey used electronic data obtained from Federal, State, and local sources for the period 1947-2007 and includes data gathered and reported from the USGS, the U.S. Environmental Protection Agency (USEPA), U.S. Department of Agriculture Forest Service (USFS), Colorado Department of Natural Resources, Colorado Department of Public Health and Environment (CDPHE), Colorado Division of Water Resources, Colorado Department of Transportation, Colorado Division of Wildlife (River Watch), Eagle Mine Superfund Site Assessment, Colorado Springs Utilities, Eagle River Water and Sanitation District, Grand River Consulting, and Advanced Sciences Inc. (table 1).

Table 1. Sources of water-related data for the Eagle River watershed.

[NA, not applicable; No., number]

\begin{tabular}{lccc}
\hline \multicolumn{1}{c}{ Agency } & $\begin{array}{c}\text { No. of } \\
\text { sites }\end{array}$ & $\begin{array}{c}\text { No. of } \\
\text { samples }\end{array}$ \\
\hline Streamflow & & \\
\hline City of Colorado Springs & 1 & NA \\
Colorado Division of Water Resources & 5 & NA \\
U.S. Geological Survey & 37 & NA \\
\hline
\end{tabular}


Table 1. Sources of water-related data for the Eagle River watershed.-Continued

[NA, not applicable; No., number]

\begin{tabular}{lrr}
\hline \multicolumn{1}{c}{ Agency } & $\begin{array}{c}\text { No. of } \\
\text { sites }\end{array}$ & $\begin{array}{c}\text { No. of } \\
\text { samples }\end{array}$ \\
\hline \multicolumn{1}{c}{ Surface-water quality } & & \\
\hline $\begin{array}{l}\text { Advanced Sciences Inc. } \\
\text { Colorado Department of Natural } \\
\text { Resources }\end{array}$ & 13 & 299 \\
$\begin{array}{l}\text { Colorado Department of Public Health } \\
\text { and Environment }\end{array}$ & 40 & 171 \\
Colorado Department of Transportation & 3 & 122 \\
Colorado Springs Utilities & 9 & 262 \\
$\begin{array}{l}\text { Division of Wildlife (River Watch) } \\
\text { Eagle Mine Superfund Site Assessment }\end{array}$ & 23 & 539 \\
Eagle River Water and Sanitation & 44 & 2,116 \\
$\begin{array}{l}\text { District } \\
\text { Grand River Consulting }\end{array}$ & 10 & 997 \\
U.S. Environmental Protection Agency & 3 & 26 \\
U.S. Forest Service & 7 & 18 \\
U.S. Geological Survey & 131 & 93 \\
\hline
\end{tabular}

\section{Relevant Publications}

Several previous studies have investigated the water quality in the ERW. The Eagle River Watershed Plan (1996) was initiated by local governments after protection of the Eagle River was identified as a top community concern through town and county planning forums and surveys (Eagle River Watershed Plan, 1996). The purpose of the plan was to outline a collaborative, local philosophy for protecting and improving water quantity, water quality, wildlife habitat, and recreational opportunities, while promoting compatible land-use practices.

The USEPA and the State of Colorado have an agreement that designates the CDPHE as the lead agency on the Eagle Mine Superfund Site project. Surface-water monitoring stations were established in 1985 at the onset of the superfund investigation. In 2009, surface-water data were collected at seven monitoring stations on the Eagle River and tributaries. Annual reports and 5-year review reports are written to summarize and analyze changes in water quality. The first 5-year review was completed in October 2000. Documents related to the superfund cleanup can be found at http://www.epa.gov/ Region8/superfund/co/eagle (January 2009) and at http://www. cdphe.state.co.us $/ \mathrm{hm} /$ rpeagle.htm (January 2009).

The USGS studied the water quantity, quality, and aquatic ecology of Gore Creek (Wynn and others, 2001). The study summarized available water quantity, water quality, and aquatic-ecology data collected from 1968 through 1998 by various agencies. Wynn and others (2001) concluded that geology and land use in the watershed affects the water quality and stream biota.

Bledsoe and others (2005) developed a report that served as an inventory and assessment of the ERW. The report concluded that the greatest threats to the ERW are flow regime changes, nutrient loading, metals loading, land-use change, and cumulative effects from recreationalists.

Rupert and Plummer (2009) studied groundwater quality, age, and susceptibility to contamination in the ERW and the corresponding relation to land use. The project developed groundwater-susceptibility maps at 1:24,000 scale and incorporated new tracers for calibrating the groundwatersusceptibility models.

Zuellig and others (2010) conducted a study to analyze invertebrate data for sites in the ERW to help identify and highlight information gaps relative to water-resource management in the watershed. These data were analyzed to assess the biological condition of selected sites using invertebrate indicators. The results were integrated with previous studies to better understand the connection between human actions (land use, water-resource management, and so on) and biological conditions in the watershed.

\section{Description of Study Area}

The Eagle River drains approximately 963 square miles $\left(\mathrm{mi}^{2}\right)$ west of the Continental Divide in central Colorado before flowing into the Colorado River (Seaber and others, 1987). Located in central Colorado west of Vail Pass, the ERW is almost entirely contained within Eagle County, with a small portion in Pitkin County. The watershed extends from the eastern boundary near Vail Pass to the western boundary near Dotsero. The headwaters of the Eagle River are located in the southernmost region of the watershed near Tennessee Pass. Major tributaries within the watershed include Gore Creek, Homestake Creek, Cross Creek, Lake Creek, and Brush Creek (fig. 1). Gore Creek drains approximately $102 \mathrm{mi}^{2}$ and flows westward 19 miles (mi) out of the Gore mountain range [highest elevation 13,200 feet (ft)] through Vail to the confluence with the Eagle River near Minturn (7,730 ft). The Eagle River headwaters flow northward from Tennessee Pass (10,000 ft) on the Continental Divide through the towns of Red Cliff and Minturn to the confluence with Gore Creek. The main stem of the Eagle River then flows westerly through the towns of Avon, Wolcott, Eagle, Gypsum, and Dotsero before flowing into the Colorado River.

The study area was divided into four subwatersheds for this analysis: (1) Upper Eagle, Eagle River including all tributaries upstream from Gore Creek; (2) Gore Creek, including all tributaries: (3) Middle Eagle, Eagle River including all tributaries downstream from Gore Creek and upstream of the USGS streamflow-gaging station near Wolcott (394220106431500); (4) Lower Eagle, Eagle River including all tributaries downstream from the USGS streamflow-gaging station near Wolcott and upstream from the Colorado River (fig 1). 


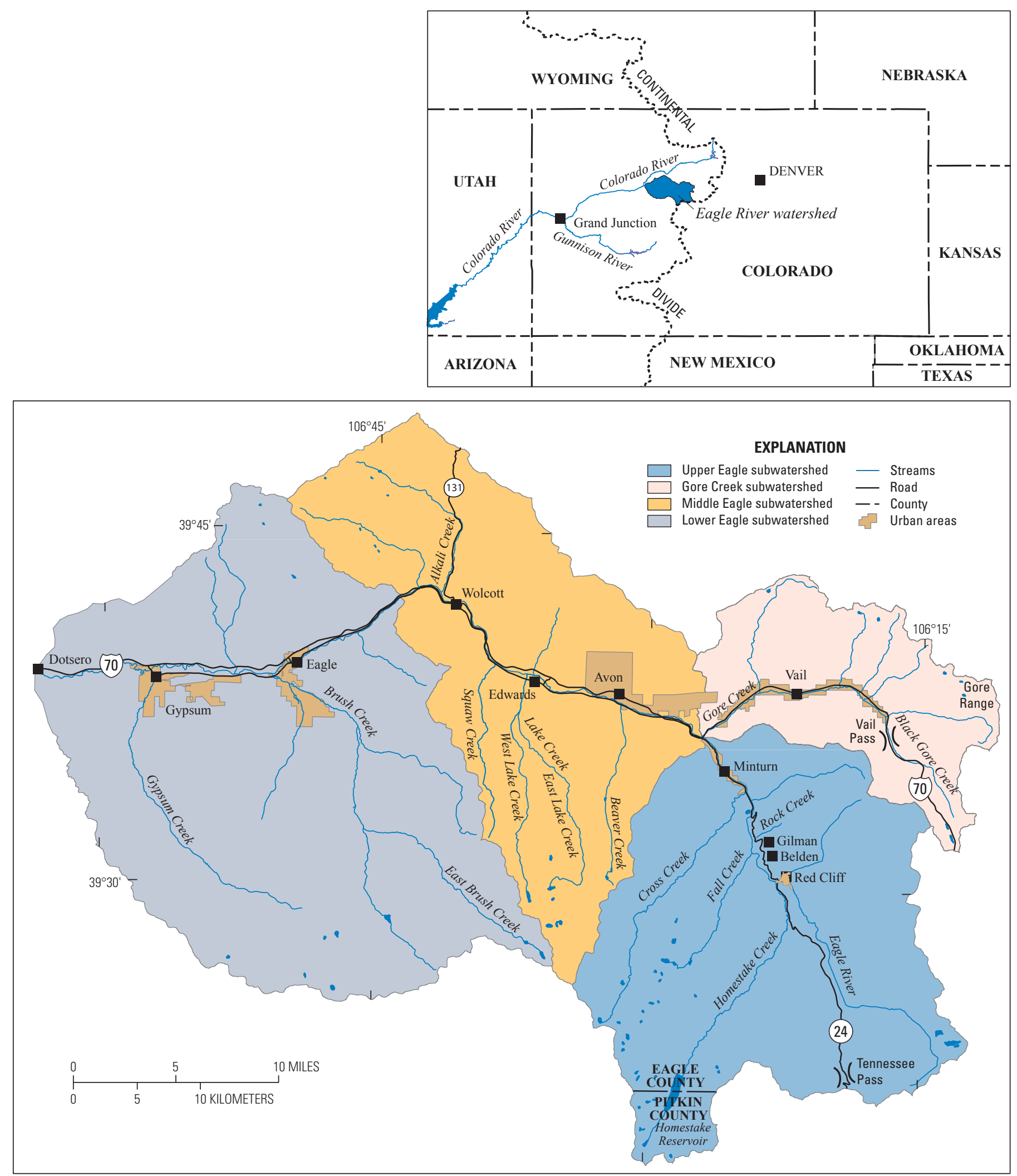

Base from U.S. Geological Survey digital data, 2007,

Universal Transverse Mercator projection

Zone 13

Figure 1. Location of the Eagle River watershed, Colorado, and location of major streams, tributaries, and urban development within the watershed. 
Monthly average temperatures in the Vail area range from 14 degrees Fahrenheit $\left({ }^{\circ} \mathrm{F}\right)$ (-10 degrees Celsius) in January to $56^{\circ} \mathrm{F}$ (13 degrees Celsius) in July. Temperatures in Eagle range from $18^{\circ} \mathrm{F}$ ( -8 degrees Celsius) in January to $67^{\circ} \mathrm{F}(19$ degrees Celsius) in July. Precipitation in the ERW ranges from 52 inches per year in the higher elevations to 11 inches in the lower valleys (fig. 2) (Western Regional Climate Center, 2009). Much of the precipitation in the watershed falls in the form of snow throughout the winter months. The annual cycle of streamflow in the ERW is marked by spring snowmelt, which causes water levels in the river to rapidly rise. Streamflow usually peaks mid June, followed by moderate flows throughout the summer months. Streamflow in autumn and winter are characterized by low flows until the snowmelt cycle starts over.
Water management within the ERW provides for municipal (including domestic and industrial), agricultural, and recreational uses. Diversion structures were constructed as early as the 1880 s to deliver water for both in and out-of-basin purposes (Bledsoe and others, 2005). Several substantial transbasin diversions direct water from the headwaters of the ERW to adjacent hydrologic basins for agriculture and to provide water for the cities east of the Continental Divide.

The population of Eagle County within the ERW in 2008 was estimated at 52,331 (U.S. Census Bureau, 2009). The largest townships in the ERW in 2004 include: Avon $(6,755)$, Gypsum $(4,944)$, Vail $(4,806)$, Eagle $(3,816)$, and Minturn $(1,115)$ (Eagle County, 2009). The population of Eagle County has increased 625 percent between 1970 and 2004 (Eagle

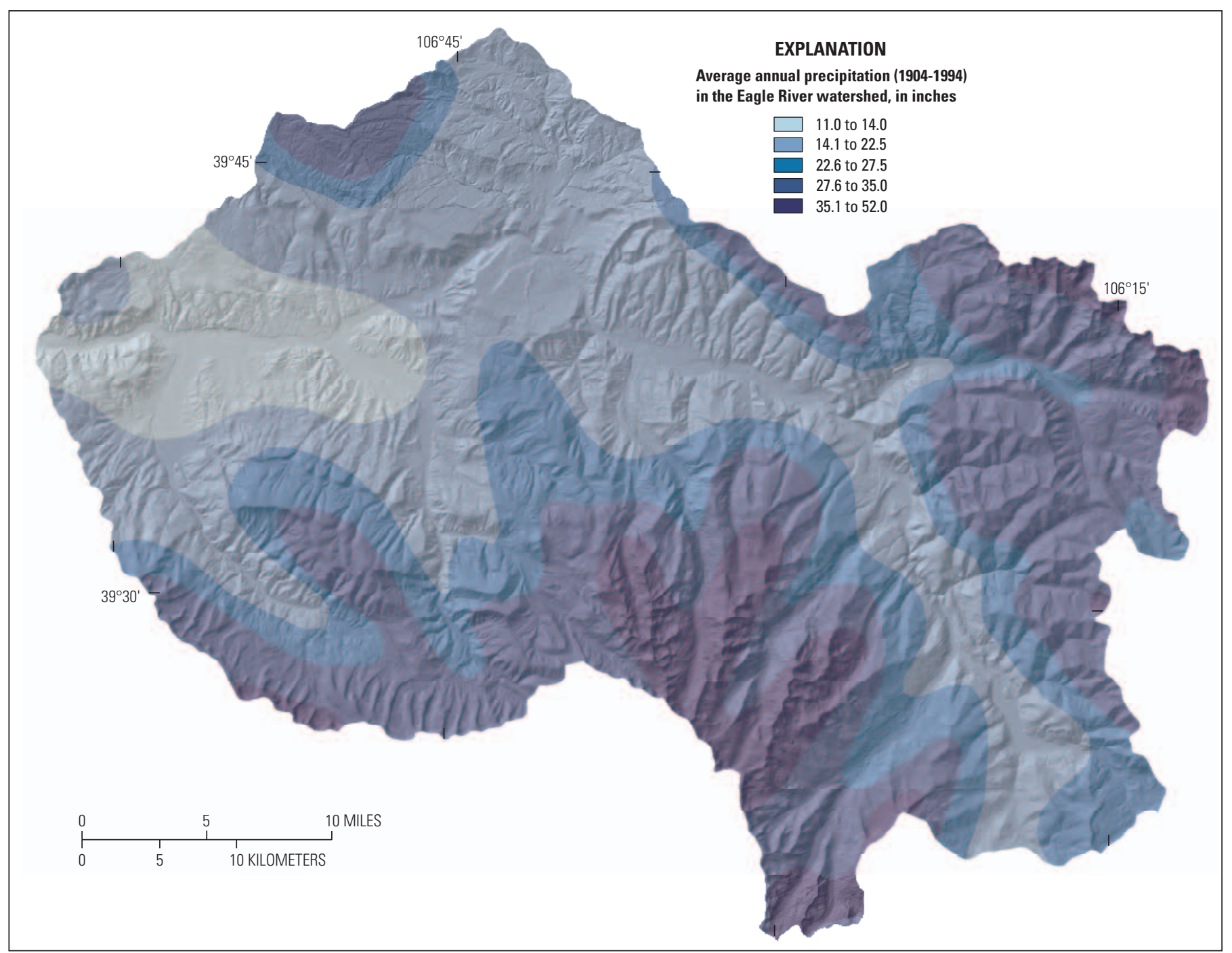

Base from U.S. Geological Survey digital data, 2007,

Universal Transverse Mercator projection

Zone 13

Figure 2. Variation in precipitation throughout the Eagle River watershed, Colorado (Western Regional Climate Center, 2009). 
County, 2009). The ERW attracts many visitors for recreational activities like fishing, hiking, camping, biking, ice climbing, and skiing.

The primary industries within the watershed are mining and recreation/tourism. Mining activities historically have included the extraction of base and precious metals from ore deposits in the Upper Eagle subwatershed as well as gypsum in the Lower Eagle subwatershed since the late 1800s (Bledsoe and others, 2005). Very little hard-rock metals mining activity presently (2009) exists in the watershed, however, gypsum mining continues near the town of Gypsum. Ski tourism, second-home development, and outdoor recreation are large contributors to the growing economy of Eagle County (Eagle County, 2009).
Land-use patterns within the ERW have been shaped by economic and development trends. The economy is shifting toward tourism, although ranching, mining, and farming still exist. Currently, within the ERW 49 percent of total lands are forested, 40 percent are shrubs, grasses, agricultural, and rangeland, 9 percent are tundra, wetlands, and barren lands, and 2 percent are developed lands (U.S. Geological Survey, 2010). Vegetation throughout the watershed is diverse. Land cover in the headwater elevations close to $10,000 \mathrm{ft}$ are dominated by rock, alpine tundra, evergreen forest, and herbaceous vegetation. Elevations at $8,000-10,000 \mathrm{ft}$ are dominated by evergreen and aspen forests. The lower regions of the Eagle River flow through an alluvial valley consisting of rangeland, shrubs, and grassland (fig. 3).

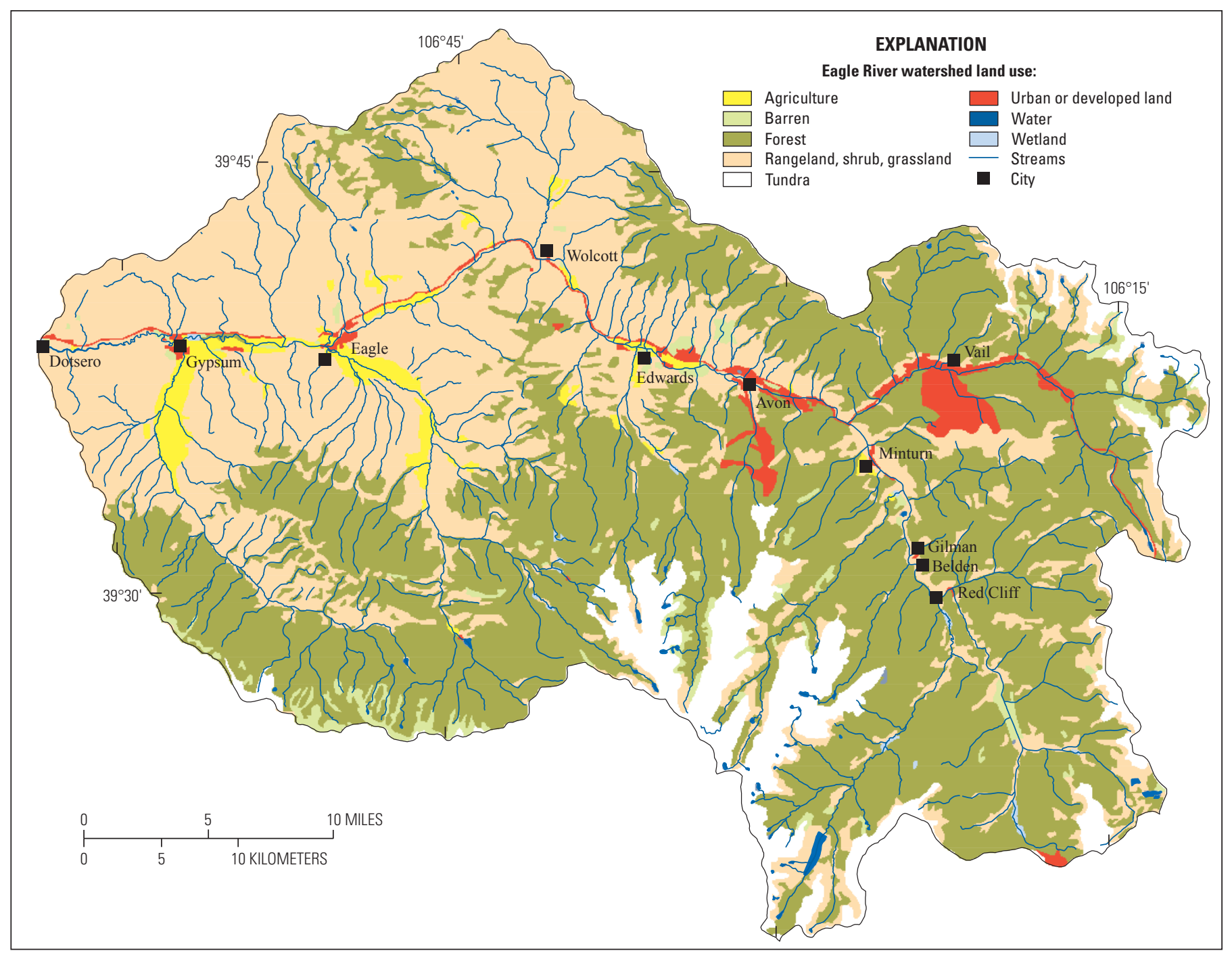

Base from U.S. Geological Survey digital data, 2007,

Universal Transverse Mercator projection

Zone 13

Figure 3. Land use in the Eagle River watershed, Colorado, 2008. 
Developed land within the watershed is increasing, which can lead to associated water-quality issues and the introduction of new contaminants (Rupert and Plummer, 2009). A major factor arising from development and the changing land use of the region is a decrease in vegetative buffers in riparian areas (Wynn and others, 2001). As runoff approaches surface waters, soil and vegetation act as filters for contaminants and nutrients, thus preventing them from entering at high concentrations. Construction of Interstate-70 (I-70) began in the early 1970s. Historically, suspended sediment associated with construction of I-70 has been a primary water-quality concern; however, recent data indicate that streambed aggradation of sediment originating from I-70 traction sanding is a greater concern (Wynn and others, 2001).

\section{Geology}

The ERW geologically is diverse and has more than a billion years of geologic history in the rock record including deposition, deformation, metamorphism, and related change. There are geologic deposits that vary from Precambrian metamorphic rocks to Quaternary alluvium and volcanic deposits (fig. 4). The wide range of geologic units contributes major ions and metals to the river system thereby affecting water quality. For example, saline shales and sandstones, along with evaporite deposits can contribute selenium and total-dissolved solids, commonly described as an estimate of salinity, to the water (Tweto, 1979; Hem, 1989). A geologically active region during the Laramide orogeny, in the southern part of the ERW, is recognized as being part of the Colorado Mineral Belt (Wilson and Sims, 2003). The Colorado Mineral Belt extends in a northeast direction from the San Juan Mountains in the southwest to just west of Boulder, Colorado, and is shown on figure 4. Igneous intrusions, hydrothermal alteration, mineralization, and various ore deposits are typically associated with the Colorado Mineral Belt. These economic ores, which can include copper, gold, lead, molybdenum, silver, tungsten, and zinc have contributed to a multibillion-dollar industry for the State (Wilson and Sims, 2003).

The ERW contains a mix of lithologies including sedimentary, metamorphic, and intermixed igneous intrusions (Tweto, 1979). The sedimentary rocks are Pennsylvanian in age conglomerate, sandstone, shale, and limestone in the eastern portion of the ERW; and Cretaceous and Pennsylvanian in age siltstone, shale, gypsum and salt-bearing deposits in the central and western areas. The northern portion of the watershed contains Cretaceous sandstone and shale deposits. These deposits include Pierre Shale and Dakota Sandstone. The Pierre Shale is a marine deposit that contains salt and has been indicated as a contributor of selenium to water supplies (Butler, 2001). The metamorphic rocks occurring along the eastern and southern portion of the ERW are Precambrian in age gneiss, schist, migmatite, and granite. The igneous rocks are Quaternary and Tertiary in age basalt flows, tuffs, and breccias, with various age intrusive deposits in the western portion of the ERW.
Three formations that are in contact with the main stem Eagle River are the Eagle Valley Evaporite, Minturn Formation, and the Belden Formation (Tweto, 1979). The Eagle Valley Evaporite, an evaporite facies of the Eagle Valley Formation, is Pennsylvanian in age and predominantly composed of gypsum, anhydrite, and interbedded siltstone and shale with thick salt deposits located sporadically underneath. The Belden Formation, also Pennsylvanian in age, is a dark-colored shale, limestone, and sandstone (Tweto, 1979). The Minturn Formation, Pennsylvanian in age, is a yellow to red sandstone and conglomerate that has interbedded layers of carbonate rocks (Tweto, 1979).

\section{Mining Activity}

Mining first began in the Gilman area in the late 1870s with the discovery of gold and silver deposits. The Gilman area is located between the towns of Minturn and Red Cliff along the Eagle River (fig. 5). Mining of lead and zinc became common in the mid 1890s. The Eagle Mine is one of the largest zinc mines in the United States, and a major domestic source of zinc (Colorado Department of Public Health and Environment and U.S. Environmental Protection Agency, 2008). Copper and silver were the last mining activities near Belden with production ending in 1984 (Colorado Department of Public Health and Environment and U.S. Environmental Protection Agency, 2008).

The Eagle Mine and associated mining wastes between Gilman and Minturn (which encompass about 235 acres), was placed on the Superfund sites list in 1986 (Colorado Department of Public Health and Environment and U.S. Environmental Protection Agency, 2008). Remediation of the Eagle Mine site began in 1988 through the relocation of processed mine wastes and contaminated soils to an on-site tailing pile. A water-treatment plant was constructed in 1990 to collect and treat mine seepage, tailing-pile groundwater, and accumulated precipitation runoff. Major remediation generally was completed by 1996. A list of major construction milestones for the Eagle Mine Superfund Site is provided in table 2.

In 2000, the first 5-year review report was written to identify human health risks and restoration of the Eagle River (Colorado Department of Public Health and Environment, 2000). The second 5-year review report was written in 2005 and concluded that remedies at the mine site continue to be protective to human health (Colorado Department of Public Health and Environment and U.S. Environmental Protection Agency, 2005). The third 5-year report was completed in 2008 and concluded that efforts to protect human health are working but additional actions are necessary to achieve protection of the aquatic ecosystem (Colorado Department of Public Health and Environment and U.S. Environmental Protection Agency, 2008).

Regional mining of gypsum and gravel near Gypsum still occurs today. The American Gypsum Plant in Gypsum produced more than 408,000 metric tons of gypsum in 1999 (Bledsoe and others, 2005). Gravel mining also is an active industry near the towns of Edwards and Gypsum. 
EXPLANATION

$\square$ Eagle River watershed Colorado Mineral Belt
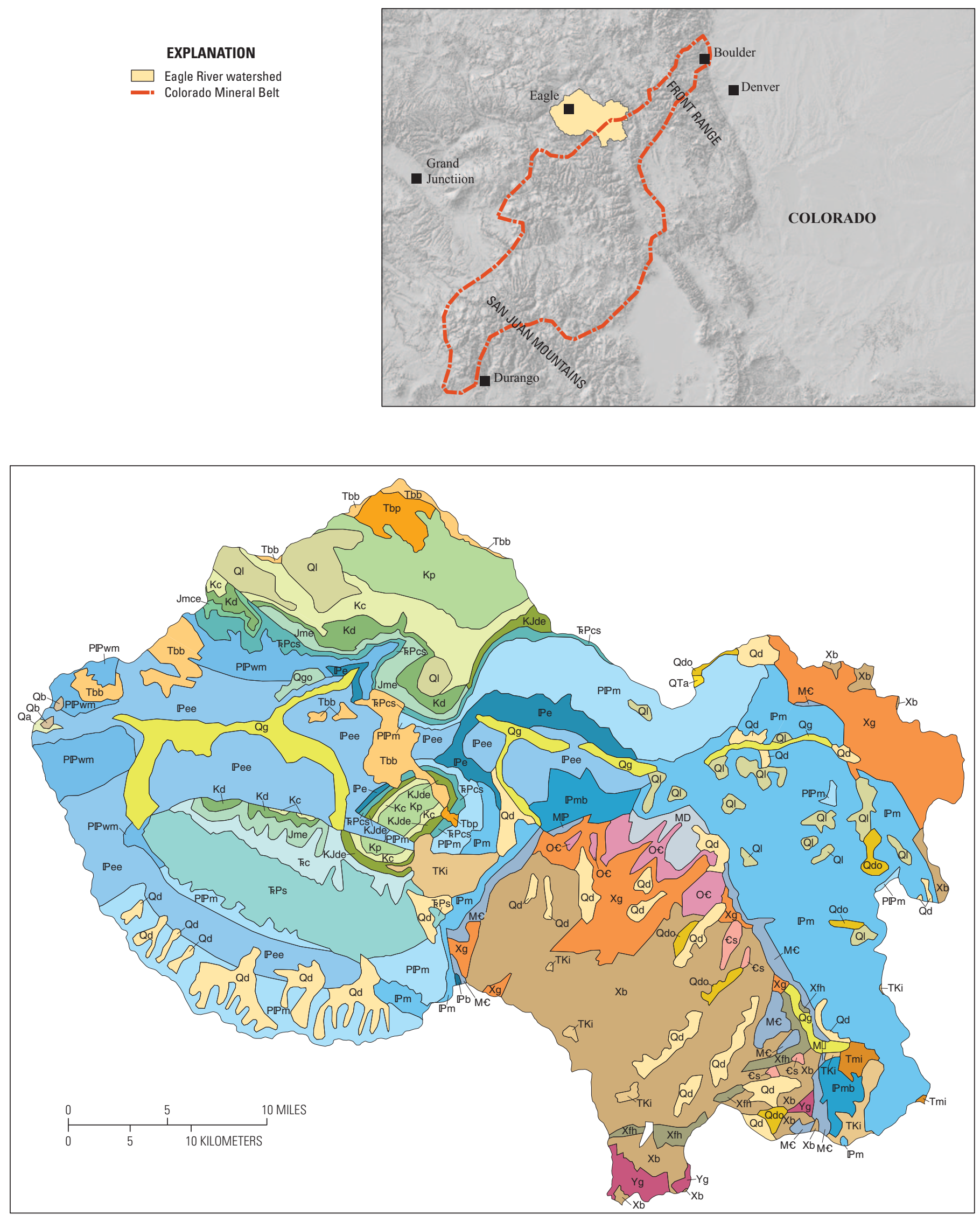

Base from U.S. Geological Survey digital data, 1992, 1:100,000, Universal Transverse Mercator projection Zone 13 


\section{EXPLANATION}

Unconsolidated Surficial Deposits and Rocks of Quaternary and Tertiary Age

\begin{tabular}{|c|}
\hline Qa \\
\hline Qg \\
\hline Qgo \\
\hline Qd \\
\hline Qdo \\
\hline Ql \\
\hline Qb \\
\hline QTa \\
\hline
\end{tabular}

Modern alluvium-Includes Holocene Piney Creek Alluvium and younger deposits

Gravels and alluviums (Pinedale and Bull Lake age)_-Includes Broadway and Louviers Alluviums

Older Gravels and alluviums (Pre-Bull Lake age)

Glacial drift of Pinedale and Bull Lake glaciations-Includes some unclassified glacial deposits

Older glacial drift (Pre-Bull Lake age)

Landslide deposits - Locally includes talus, rock-glacier, and thick colluvium deposits

Basalt flows (age less than 1.8 million years)

Ancient alluvium - In isolated patches that may not all be of the same age

\section{Sedimentary Rocks of Tertiary Age}

Browns Park Formation-Sandstone and siltstone, west of Park Range

Ingneous Rocks of Teriary and Cretaceous Age

\begin{tabular}{|l|}
\hline Tbb \\
\hline Tmi \\
\hline TKi
\end{tabular}

Basalt flows and associated tuff, breccia, and conglomerate of late volcanic bimodal suite

Middle Tertiary intrusive rocks (age 20-40 million years) Intermediate to felsic compositions

Laramide intrusive rocks (age 20-72 million years)

Sedimentary Rocks of Cretaceous and Jurassic Age

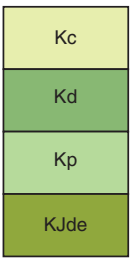

Colorado Group-Consists of Niobrara Formation and either Benton Shale or Carlile, Greenhorn, and Graneros Formations

Dakota Sandstone or Group

Pierre Shale, undivided

Dakota, Morrison, and Entrada Formations

\section{Sedimentary rocks of Jurassic Age}

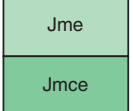

Morrison Formation and Entrada Sandstone

Morrison, Curtis, and Entrada Formations

Sedimentary Rocks of Triassic and Permian Age

\begin{tabular}{|c|}
\hline $\mathrm{kC}$ \\
\hline $\mathrm{kPS}$ \\
\hline $\mathrm{kPCS}$ \\
\hline
\end{tabular}

Chinle Formation—Red siltstone, sandstone, and limestone pellet conglomerate

State Bridge Formation—Red and orange silstone and sandstone

Chinle and State Bridge Formations, undivided

Sedimentary Rocks of Permian and Pennsylvania Age

$\mathrm{PPm}$

PPwm and local limestone

Weber Sandstone and Maroon Formation

\section{Sedimentary Rocks of Pennsylvanian Age}

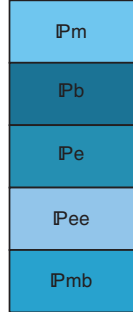

Minturn Formation-Arkosic sandstone, conglomerate, shale, and limestone

Belden Formation—shale, limestone, and sandstone

Eagle Valley formation-Siltstone, shale, and gypsum

Eagle Valley Evaporite-Gypsum, siltstone and shale, anhydrite, salt, present in deep borings

Minturn and Belden Formations

Sedimentary Rocks of Pre-Pennsylvania Paleozoic Age

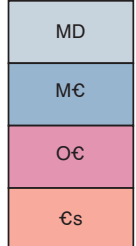

Leadville Limestone

Leadville, Gilman, Dyer, Parting, Fremont, Harding, Manitou, Dotsero, Peerless, and Sawatch Formations

Ordovician rocks

Sawatch Formation-Locally includes Peerless Formation

Sedimentary, Metamorphic, and Igneous Rocks of Precambrian Age

Biotite gneiss, schist, and migmatite-Locally contains minor hornblende gneiss, quartzite, and marble

Felsic and hornblende gneisses - Includes metabasalt, metatuff, and interbedded metagraywacke

Granitic rocks 1,400 million years

Granitic rocks 1,700 million years

Figure 4. Surficial geology of the Eagle River watershed, Colorado (from Tweto, 1979). 
Table 2. Eagle Mine Superfund Site major construction milestones (Colorado Department of Public Health and Environment and U.S. Environmental Protection Agency, 2008).

\begin{tabular}{ll}
\hline Major construction milestones & Date completed \\
\hline Rex Flats tailing removal & 1988,1996 \\
Roaster Pile \#5 removal & 1988 \\
Consolidated tailings pile groundwater extraction system completed & 1989 \\
Roaster Pile \#4 removal & 1989 \\
Roaster Piles \#1, 2, 3 removal & 1989 \\
Package water treatment plant installed & 1990 \\
Roaster Gulch sediment and sediment basin removal & $1990,1992,1994$ \\
Old tailings pile tailing removal & 1991 \\
Pipeline trestle tailing removal & 1991 \\
Customized water treatment plant constructed & 1991 \\
Sludge Press added to water treatment plant operation & 1994 \\
Maloit Park soil removal & 1995,1996 \\
Consolidated tailings pile cap completed & 1996 \\
Belden "concentrate" removal & 1997 \\
\hline
\end{tabular}




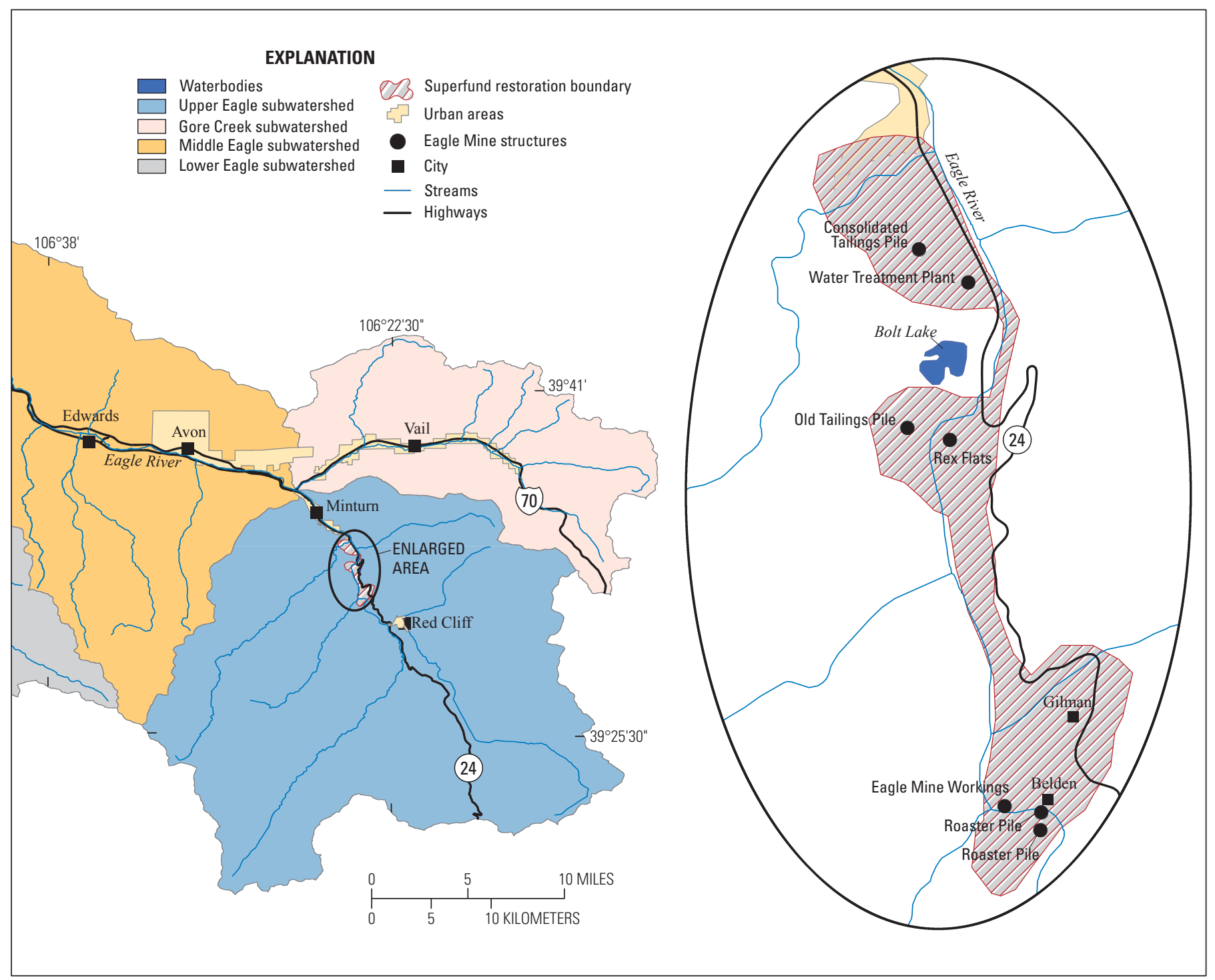

Base from U.S. Geological Survey digital data, 2007,

Universal Transverse Mercator projection

Zone 13

Figure 5. Location of hard-rock mining and related superfund remediation in the Eagle River watershed, Colorado, 2008.

\section{Data Sources, Compilation, Review, and Analysis}

The availability of multiple digital surface-water data sources within the ERW provides extensive information to support an assessment of the surface-water conditions within the watershed; however, these data sources are the product of multiple data-collection objectives, collection methodologies, and temporal scales within the watershed. Details relevant to the sources of data used in the analysis, methods used to generate the analysis dataset structure, and limitations inherent in the data analysis are presented.

\section{Surface-Water Quantity}

Available surface-water quantity data were obtained from the USGS National Water Information System (NWIS, URL: http://waterdata.usgs.gov/nwis) and the City of Colorado Springs (URL: http://www.springsgov.com/ Index.aspx). Streamflow-gaging stations have monitored surface-water quantity in the ERW at up to 39 different locations in the watershed since water year 1911 (fig. 6, table 3). In 2009, the USGS monitored surface-water quantity (streamflow) at 14 sites, and the City of Colorado Springs operated a streamflow gaging-station on the outflow from Homestake Reservoir. The Homestake Reservoir 


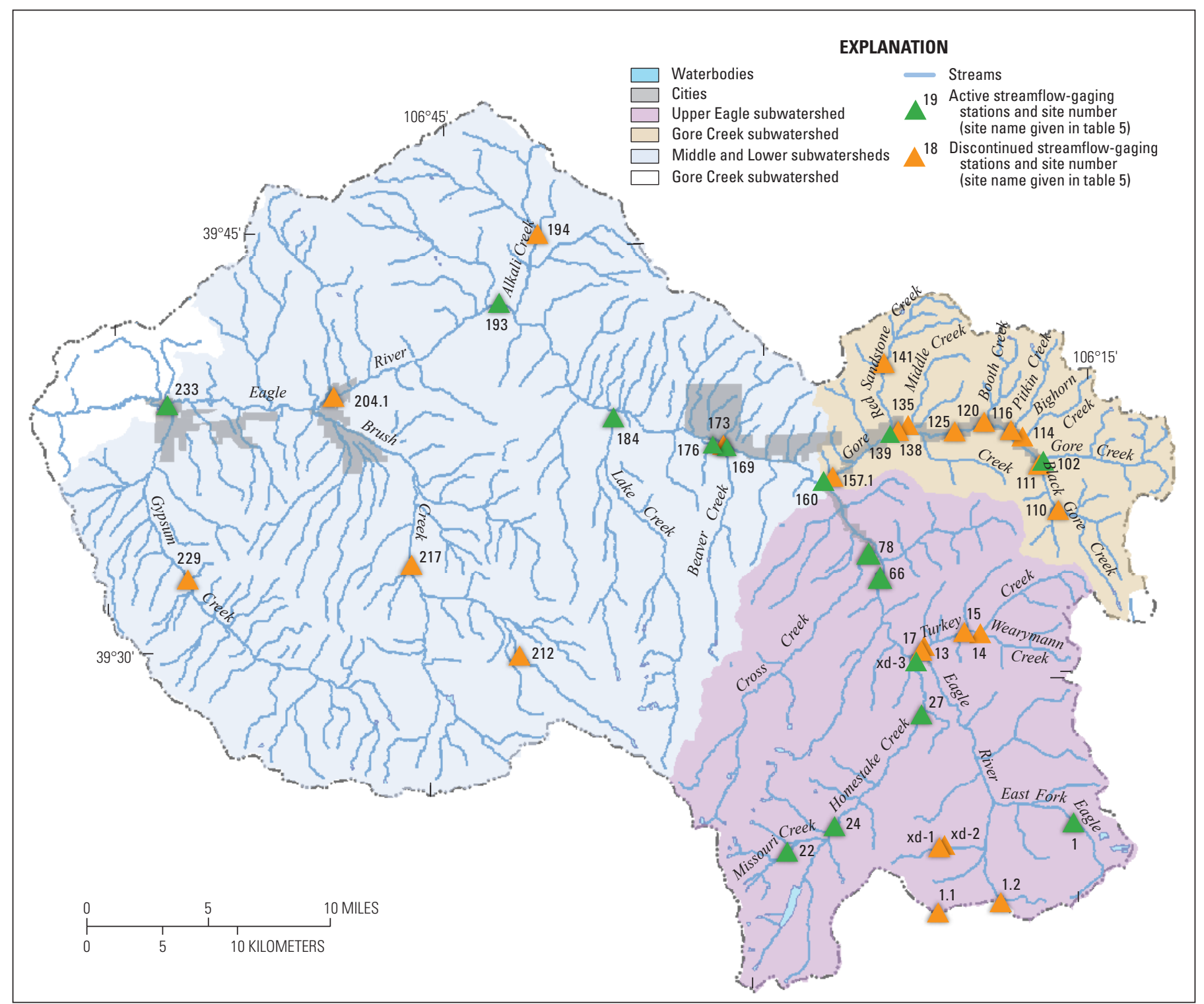

Base from U.S. Geological Survey digital data, 2007

Universal Transverse Mercator projection

Zone 13

Figure 6. Streamflow-gaging station locations and streamflow distribution for selected streams in the Eagle River watershed, Colorado, by subwatershed.

outfall is used in this report for information pertaining to water management and flow augmentation within the ERW and is not shown in figure 6 or in table 3 . No other streamflow-gaging stations currently (2009) are operated by other agencies within the ERW.

A summary of streamflow statistics for selected streamflow-gaging stations is available in table 4. Median annual maximum daily streamflow and median annual minimum 7-day mean streamflow were calculated from available annual summary statistics between 1968-2008 to provide estimates of typical high and low-flow conditions (NWIS, URL: http:// waterdata.usgs.gov/nwis). The mean annual runoff, daily mean streamflow exceedance, and flood frequency statistics are of value to water managers because these data provide a historical summary that aids in the prediction of recurrence of highand low-flow conditions.

Characterization of the prevailing climatic conditions for sites was done using a Locally Weighted Scatterplot Smoothing (LOWESS Smooth) of the annual mean streamflow at selected sites (Helsel and Hirsh, 1992). Selection of the appropriate smoothing factor was completed iteration until the best fit was obtained for each of the selected sites. 
Table 3. Hydrologic characteristics for streamflow-gaging stations in the Eagle River watershed.

[no., number; USGS, U.S. Geological Survey; CDWR, Colorado Division of Water Resources; CO, Colorado; water year, continuous period from October 1 through September 30 with the year of designation determined by the ending calendar year; --, no data; $\mathrm{xd}$, transmountain diversion]

\begin{tabular}{|c|c|c|c|c|c|c|c|}
\hline Site no. & Site name & $\begin{array}{l}\text { Source } \\
\text { agency }\end{array}$ & $\begin{array}{c}\text { Site } \\
\text { identifier }\end{array}$ & $\begin{array}{l}\text { Period of record } \\
\text { stored in a database, } \\
\text { in water years }{ }^{1}\end{array}$ & $\begin{array}{c}\text { Drainage area, } \\
\text { in square miles }{ }^{2}\end{array}$ & $\begin{array}{l}\text { Mean annual } \\
\text { streamflow, in cubic } \\
\text { feet per second }{ }^{3}\end{array}$ & $\begin{array}{l}\text { Streamflow-gaging } \\
\text { station status, } \\
\text { as of water year } 2009\end{array}$ \\
\hline 1 & East Fork Eagle River near Climax, CO & USGS & 09061600 & $2002-08$ & 7.78 & 4.77 & Active \\
\hline $\mathrm{xd}-1$ & Wurtz Extension Ditch near Tennessee Pass, CO & CDWR & WUREXDCO & 1967-91 & -- & -- & Discontinued \\
\hline $\mathrm{xd}-2$ & Wurtz Extension & CDWR & WUREXTCO & 1991-2006 & -- & -- & Discontinued \\
\hline 1.1 & Wurtz Ditch near Tennessee Pass, CO & $\begin{array}{l}\text { USGS } \\
\text { CDWR }\end{array}$ & $\begin{array}{l}09062500 \\
\text { WURDITCO }\end{array}$ & $\begin{array}{l}1948-67 \\
1947-2006\end{array}$ & -- & -- & Discontinued \\
\hline 1.2 & Ewing Ditch at Tennessee Pass, CO & $\begin{array}{l}\text { USGS } \\
\text { CDWR }\end{array}$ & $\begin{array}{l}09062000 \\
\text { EWIDITCO }\end{array}$ & $\begin{array}{l}1948-67 \\
1947-2006\end{array}$ & -- & -- & Discontinued \\
\hline 13 & Eagle River at Red Cliff, CO & USGS & 09063000 & $1911-25,1945-2008$ & 70.0 & 45.3 & Active \\
\hline$x d-3$ & Eagle River at Red Cliff Plus Columbine, Ewing, and Wurtz 5 & USGS & 09063001 & $1947-1967$ & 70.0 & $48.0^{4}$ & Discontinued \\
\hline 14 & Wearyman Creek near Red Cliff, CO & USGS & 09063200 & 1965-2008 & 9.53 & 8.24 & Discontinued \\
\hline 15 & Turkey Creek near Red Cliff, CO & USGS & 09063400 & 1964-2008 & 23.8 & 21.7 & Discontinued \\
\hline 16.1 & Turkey Creek at Red Cliff, CO & USGS & 09063500 & 1914-21, 1945-56 & 29.4 & $28.6^{4}$ & Discontinued \\
\hline 22 & Missouri Creek near Gold Park, CO & USGS & 09063900 & 1972-2008 & 6.39 & 7.37 & Active \\
\hline 24 & Homestake Creek at Gold Park, CO & USGS & 09064000 & $1948-54,1972-2008$ & 36.0 & 27.0 & Active \\
\hline 27 & Homestake Creek near Red Cliff, CO & USGS & 09064500 & $1911-18,1945-2008$ & 58.2 & 41.6 & Active \\
\hline 66 & Eagle River near Minturn, $\mathrm{CO}$ & USGS & 09064600 & $1990-2008$ & 186 & 122 & Active \\
\hline 78 & Cross Creek near Minturn, $\mathrm{CO}$ & USGS & 09065100 & $1957-64,1968-2008$ & 34.2 & 51.9 & Active \\
\hline 102 & Gore Creek at Upper Station, near Minturn, CO & USGS & 09065500 & $1948-56,1964-2008$ & 14.4 & 29.7 & Active \\
\hline 110 & & $\begin{array}{l}\text { CDWR } \\
\text { USGS }\end{array}$ & GORMINCO & $1963-66$ & 14.7 & -- & Discontinued \\
\hline $\begin{array}{l}110 \\
111\end{array}$ & $\begin{array}{l}\text { Black Gore Creek near Minturn, CO } \\
\text { Black Gore Creek near Vail, CO }\end{array}$ & $\begin{array}{l}\text { USGS } \\
\text { USGS }\end{array}$ & $\begin{array}{l}09066000 \\
09066050\end{array}$ & $\begin{array}{l}1948-56,1964-2008 \\
1974-80\end{array}$ & $\begin{array}{l}12.6 \\
19.6\end{array}$ & $\begin{array}{l}16.7 \\
28.7^{4}\end{array}$ & $\begin{array}{l}\text { Discontinued } \\
\text { Discontinued }\end{array}$ \\
\hline 114 & Bighorn Creek near Minturn, CO & USGS & 09066100 & $1964-2008$ & 4.54 & 9.77 & Discontinued \\
\hline 116 & Pitkin Creek near Minturn, CO & USGS & 09066150 & $1967-2008$ & 5.32 & 11.6 & Discontinued \\
\hline 120 & Booth Creek near Minturn, CO & USGS & 09066200 & 1965-2008 & 6.02 & 11.8 & Discontinued \\
\hline 125 & Gore Creek at Vail, $\mathrm{CO}$ & USGS & 09066250 & 1974-79 & 57.3 & $95.5^{4}$ & Discontinued \\
\hline 135 & Middle Creek near Minturn, CO & USGS & 09066300 & 1965-2008 & 5.94 & 5.63 & Discontinued \\
\hline 138 & Gore Creek, Lower Station at Vail, CO & USGS & 09066310 & 1988-99 & 77.1 & $118^{4}$ & Discontinued \\
\hline 139 & Gore Creek above Red Sandstone Creek at Vail, CO & USGS & 09066325 & $2000-08$ & 77.1 & 101 & Active \\
\hline 141 & Red Sandstone Creek near Minturn, CO & USGS & 09066400 & 1964-2008 & 7.32 & 8.85 & Discontinued \\
\hline 157.1 & Gore Creek near Minturn, $\mathrm{CO}$ & USGS & 09066500 & $1945-56$ & 101 & $129^{4}$ & Discontinued \\
\hline 160 & Gore Creek at Mouth, near Minturn, $\mathrm{CO}$ & USGS & 09066510 & 1996-2008 & 102 & 128 & Active \\
\hline 169 & Beaver Creek at Avon, $\mathrm{CO}$ & USGS & 09067000 & 1974-2008 & 14.8 & 13.0 & Active \\
\hline 173 & Eagle River At Avon, CO & USGS & 09067005 & 1989-99 & 395 & $386^{4}$ & Discontinued \\
\hline 176 & Eagle River below Wastewater Treatment Plant at Avon, CO & USGS & 09067020 & $2000-08$ & 402 & 317 & Active \\
\hline 184 & Lake Creek near Edwards, CO & USGS & 09067200 & 1994-2008 & 49.0 & 57.6 & Active \\
\hline 194 & Alkali Creek near Wolcott, $\mathrm{CO}$ & USGS & 09067300 & $1959-65$ & 27.3 & $2.13^{4}$ & Discontinued \\
\hline 201 & Eagle River below Milk Creek near Wolcott, CO & USGS & 394220106431500 & 2006-2008 & 600 & -- & Active \\
\hline 204.1 & Eagle River at Eagle, $\mathrm{CO}$ & USGS & 09067500 & $1912-24$ & 629 & $676^{3}$ & Discontinued \\
\hline 212 & East Brush Creek at Yeoman Park, near Eagle, CO & USGS & 09067700 & $1966-72$ & 9.74 & $14.9^{4}$ & Discontinued \\
\hline 217 & Brush Creek near Eagle, CO & USGS & 09068000 & 1951-72 & 71.4 & $44.1^{4}$ & Discontinued \\
\hline 229 & Gypsum Creek near Gypsum, CO & USGS & 09069500 & $1951-55,1966-72$ & 62.7 & $32.4^{4}$ & Discontinued \\
\hline 233 & Eagle River below Gypsum, $\mathrm{CO}$ & USGS & 09070000 & $1947-2008$ & 944 & 566 & Active \\
\hline
\end{tabular}

'Water year from October $1-$ September 30.

'Data obtained from USGS National Water Information System: Web Interface URL: http://waterdata.usgs.gov/nwis.

${ }^{3}$ Mean annual streamflow for period of record; data obtained from USGS Water-Data Report 2007 unless otherwise indicated.

${ }^{4}$ Mean annual streamflow for period of record calculated from USGS National Water Information System: Web Interface URL: http://waterdata.usgs.gov/nwis.

${ }^{5}$ Does not have a physical location, streamflow is calculated using nearby gages. 
Table 4. Summary of the mean annual runoff, median annual maximum daily streamflow, annual flood frequency, and low-flow statistics for selected sites in the Eagle River watershed.

[no., number; \%, percent of time per year; --, no data; yr, year]

\begin{tabular}{|c|c|c|c|c|c|c|c|c|c|c|c|}
\hline \multirow[t]{2}{*}{$\begin{array}{l}\text { Site } \\
\text { no. }\end{array}$} & \multirow[t]{2}{*}{$\begin{array}{l}\text { Period of record, } \\
\text { in water years }\end{array}$} & \multirow{2}{*}{$\begin{array}{l}\text { Mean } \\
\text { annual runoff, } \\
\text { in acre-feet }^{2}\end{array}$} & \multicolumn{3}{|c|}{$\begin{array}{l}\text { Daily mean streamflow that was exceeded } \\
\text { the specified percent of time per year, } \\
\text { in cubic feet per second }{ }^{2}\end{array}$} & \multirow{2}{*}{$\begin{array}{l}\text { Median annual } \\
\text { maximum daily } \\
\text { streamflow, in cubic } \\
\text { feet per second }{ }^{3}\end{array}$} & \multicolumn{4}{|c|}{$\begin{array}{l}\text { Peak flood-frequency } \\
\text { recurrence interval, } \\
\text { in cubic feet per second }\end{array}$} & \multirow{2}{*}{$\begin{array}{l}\text { Median annual } \\
\text { minimum 7-day mear } \\
\text { streamflow, in cubic } \\
\text { feet per second }^{3}\end{array}$} \\
\hline & & & $10 \%$ & $50 \%$ & $90 \%$ & & $2 \mathrm{yr}$ & $5 \mathrm{yr}$ & $10 \mathrm{yr}$ & $100 \mathrm{yr}$ & \\
\hline 13 & $1911-25,1945-2008$ & 32,800 & 122 & 15 & 9.0 & 251 & -- & -- & -- & -- & 7.0 \\
\hline 14 & $1965-2008$ & 5,970 & 23 & 2.4 & 1.2 & 69 & 83 & 124 & 150 & 229 & 1.0 \\
\hline 15 & 1964-2008 & 15,730 & 33 & 5.8 & 2.8 & 170 & 236 & 370 & 460 & 743 & 2.6 \\
\hline 22 & 1972-2008 & 5,340 & 18 & 2.4 & .60 & 63 & -- & -- & -- & -- & .6 \\
\hline 24 & 1948-54, 1972-2008 & 19,560 & 59 & 12 & 4.4 & 177 & -- & -- & -- & -- & 4.0 \\
\hline 27 & 1911-18, 1945-2008 & 30,170 & 109 & 17 & 6.2 & 275 & 805 & 997 & 1,107 & 1,388 & 5.7 \\
\hline 66 & 1990-2008 & 88,490 & 339 & 48 & 25 & 740 & -- & -- & -- & -- & 21 \\
\hline 78 & 1957-64, 1968-2008 & 37,570 & 174 & 12 & 2.4 & 415 & -- & -- & -- & -- & 2.4 \\
\hline 102 & 1948-56, 1964-2008 & 21,520 & 99 & 7.1 & 2.5 & 250 & 334 & 441 & 506 & 681 & 2.3 \\
\hline 110 & 1948-56, 1964-2008 & 12,100 & 50 & 3.9 & 2.0 & 164 & 215 & 274 & 308 & 399 & 1.9 \\
\hline 114 & 1964-2008 & 7,080 & 32 & 2.4 & .70 & 75 & 86 & 132 & 163 & 259 & .7 \\
\hline 116 & $1967-2008$ & 8,410 & 37 & 3.4 & 1.2 & 78 & -- & -- & -- & -- & 1.0 \\
\hline 120 & 1965-2008 & 8,550 & 40 & 2.3 & .77 & 106 & 143 & 182 & 205 & 266 & .7 \\
\hline 135 & 1965-2008 & 4,080 & 19 & .95 & .22 & 55 & 74 & 107 & 128 & 188 & .2 \\
\hline 141 & 1964-2008 & 6,410 & 28 & 1.8 & .88 & 84 & 11 & 156 & 183 & 262 & .8 \\
\hline 169 & 1974-2008 & 9,450 & 38 & 4.5 & 2.1 & 82 & -- & -- & -- & -- & 1.9 \\
\hline 233 & $1947-2008$ & 409,900 & 1,540 & 242 & 158 & 3,425 & 3,870 & 5,100 & 5,840 & 7,830 & 135 \\
\hline
\end{tabular}

${ }^{1}$ Sites names are shown in table 3 .

${ }^{2}$ U.S. Geological Survey, 2007.

${ }^{3}$ Data calculated from available continuous data, 1968 to 2008, from U.S. Geological Survey National Water Information System: Web Interface database: URL: http:// waterdata.usgs.gov/co/nwis/sw.

${ }^{4}$ Ries III and others, 2008. 
Table 5. Historic and current surface-water quantity and quality sites in the Eagle River watershed, Colorado.

[no., number; USGS, U.S. Geological Survey; CDPHE, Colorado Department of Public Health and Environment; CO, Colorado; WWTP, wastewater treatment plant; CSU, Colorado Springs Utilities; GRC, Grand River Consulting, EMSSA, Eagle Mine Superfund Site Assessment; DOW-RW, Division of Wildlife, River Watch; CDNR, Colorado Division of Natural Resources; ASI, Advanced Sciences Incorporated; CDOT, Colorado Department of Transportation; ERWSD, Eagle River Water and Sanitation District; USFS, U.S. Forest Service; USEPA, U.S. Environmental Protection Agency; FP, field properties; MI, major ions; TE, trace elements; O, other constituents; AE, aquatic ecology; S, sediment; N, nutrients; R, radionuclide]

\begin{tabular}{|c|c|c|c|c|c|c|c|c|c|}
\hline $\begin{array}{l}\text { Site } \\
\text { no. }\end{array}$ & Site name & $\begin{array}{c}\text { Data } \\
\text { sources }\end{array}$ & $\begin{array}{c}\text { Site } \\
\text { identifiers }\end{array}$ & Latitude & Longitude & Type of data & $\begin{array}{c}\text { Period of } \\
\text { discharge record } \\
\text { (water year) }\end{array}$ & $\begin{array}{l}\text { Period of water- } \\
\text { quality record } \\
\text { (calendar year) }\end{array}$ & $\begin{array}{l}\text { No. of } \\
\text { samples }\end{array}$ \\
\hline 1 & East Fork Eagle River near Climax, $\mathrm{CO}$ & USGS & 09061600 & 39.41027 & -106.24975 & FP & $2002-08$ & $2002-2004$ & 18 \\
\hline 2 & $\begin{array}{l}\text { East Fork Eagle River above Sheep Gl near Camp } \\
\text { Hale, CO }\end{array}$ & USGS & 392440106150400 & 39.41110 & -106.25169 & FP, MI, TE & & $1972-1973$ & 2 \\
\hline \multirow[t]{2}{*}{3} & East Fork Eagle River near Red Cliff, CO & USGS & 392511106164000 & 39.41971 & -106.27836 & $\mathrm{O}, \mathrm{FP}, \mathrm{MI}, \mathrm{AE}, \mathrm{S}, \mathrm{N}, \mathrm{TE}$ & & 1996-2007 & 79 \\
\hline & & CDPHE & 392511106164000 & & & FP, MI, TE & & 1979 & 4 \\
\hline 4 & Cataract Creek at mouth near Camp Hale, CO & USGS & 392514106165800 & 39.42055 & -106.28293 & FP, MI, TE & & 1972 & 1 \\
\hline 5 & East Fork Eagle River, $\mathrm{CO}$ & CSU & E8_EFORK & 39.42028 & -106.29944 & FP, TE, MI, AE & & 1990 & 1 \\
\hline 6 & East Fork Eagle River above Camp Hale, CO & GRC & EAG1 EFE & 39.41944 & -106.30333 & FP, MI, N, TE & & 1997 & 5 \\
\hline 7 & East Fork Eagle River at mouth below Climax, $\mathrm{CO}$ & USGS & $39251 \overline{8} 106184700$ & 39.42112 & -106.31212 & $\mathrm{O}, \mathrm{FP}, \mathrm{TE}, \mathrm{MI}, \mathrm{N}$ & & 1976 & 2 \\
\hline 8 & South Fork Eagle River above Camp Hale ,CO & GRC & EAG3_SFE & 39.41222 & -106.31583 & FP, MI, N, TE & & 1997 & 5 \\
\hline \multirow[t]{2}{*}{9} & South Fork Eagle River above East Fork Eagle & USGS & $39251 \overline{3} 106184500$ & 39.42027 & -106.31294 & $\mathrm{FP}, \mathrm{AE}$ & & $1996-1997$ & 2 \\
\hline & River near Red Cliff, CO & CDPHE & 392513106184500 & & & FP, MI, TE & & 1979 & 4 \\
\hline 10 & Resolution Creek near Eagle River, CO & GRC & EAG2_RESCK & 39.44778 & -106.31944 & FP, MI, N, TE & & 1997 & 5 \\
\hline 11 & Eagle River at Camp Hale, CO & CSU & E7_CHALE & 39.44833 & -106.32750 & $\mathrm{O}, \mathrm{FP}, \mathrm{TE}, \mathrm{MI}, \mathrm{AE}$ & & $1996-2002$ & 15 \\
\hline 12 & Eagle River below Camp Hale, CO & GRC & EAG4_BCH & 39.46056 & -106.32944 & FP, MI, N, TE & & 1997 & 6 \\
\hline \multirow[t]{2}{*}{13} & Eagle River at Red Cliff, CO & USGS & 09063000 & 39.50838 & -106.36598 & AE, O, FP, MI, S, N, TE & $1911-25,1945-2008$ & $1958-2007$ & 501 \\
\hline & & CDPHE & 09063000 & & & FP, TE, MI, AE, N & & 1979-1997 & 93 \\
\hline 14 & Wearyman Creek near Red Cliff, CO & USGS & 09063200 & 39.52238 & -106.32357 & FP & 1965-2008 & 1964-2004 & 345 \\
\hline 15 & Turkey Creek near Red Cliff, CO & USGS & 09063400 & 39.52276 & -106.33614 & $\mathrm{FP}$ & $1964-2008$ & $1963-2004$ & 418 \\
\hline 16 & Turkey Creek at Red Cliff intake near Red Cliff, $\mathrm{CO}$ & USGS & 393109106210600 & 39.51915 & -106.35225 & $\mathrm{O}, \mathrm{FP}, \mathrm{MI}, \mathrm{AE}, \mathrm{N}, \mathrm{TE}$ & & 1976 & 2 \\
\hline 17 & Turkey Creek at Red Cliff, CO & CDPHE & 12590 & 39.51402 & -106.36687 & FP, MI, AE, S, N, TE & & 2001 & 4 \\
\hline \multirow[t]{5}{*}{20} & Eagle River above Homestake Creek, CO & CDPHE & E-1R & 39.50833 & -106.37656 & FP, MI, TE & & 1979 & 4 \\
\hline & & EMSSA & E-1R & 39.50834 & -106.37640 & $\mathrm{O}, \mathrm{FP}, \mathrm{MI}, \mathrm{N}, \mathrm{TE}$ & & 1995-2002 & 17 \\
\hline & & DOW-RW & 3284 & 39.50833 & -106.37691 & FP, MI, TE & & $1992-2001$ & 10 \\
\hline & & DOW-RW & 38 & 39.50825 & -106.37584 & FP, MI, TE & & 1991 & 3 \\
\hline & & CSU & E7_AHMCR & & & FP & & 1987 & 1 \\
\hline 22 & Missouri Creek near Gold Park, CO & USGS & 09063900 & 39.38986 & -106.46961 & FP & 1972-2008 & $1972-2004$ & 244 \\
\hline 24 & Homestake Creek at Gold Park, CO & USGS & 09064000 & 39.40554 & -106.43336 & FP & $1948-54,1972-2008$ & 1972-2004 & 250 \\
\hline \multirow[t]{4}{*}{27} & Homestake Creek near Red Cliff, CO & USGS & 09064500 & 39.47332 & -106.36781 & AE, O, FP, MI, S, N, TE & 1911-1918, 1945-2008 & 1960-2004 & 417 \\
\hline & & CDPHE & 09064500 & 39.47313 & -106.36833 & FP, TE, R, MI, AE, S, N, O & & $1970-2001$ & 62 \\
\hline & & CDPHE & 12564 & 39.47306 & -106.36861 & FP MI, AE, S, N, TE & & 2001-2002 & 5 \\
\hline & & GRC & EAG5_HSC & & & FP, MI, N, TE & & 1997 & 5 \\
\hline 28 & $\begin{array}{l}\text { Eagle River below Homestake Creek near Red } \\
\text { Cliff, CO }\end{array}$ & USGS & 393030106224700 & 39.50833 & -106.37972 & $\mathrm{AE}, \mathrm{O}, \mathrm{FP}, \mathrm{MI}, \mathrm{S}, \mathrm{N}, \mathrm{TE}$ & & 1983-2003 & 15 \\
\hline 29 & Eagle River below Homestake Creek and at B & CSU & E4 BHMCR & 39.51194 & -106.38459 & FP, MI, N & & 1987 & 6 \\
\hline 30 & Eagle River above Peterson Gulch & CDPHE & EAGLE07 & 39.51416 & -106.38684 & FP, MI, TE & & 1979 & 5 \\
\hline 31 & Peterson Creek at mouth & USGS & 393057106232301 & 39.51570 & -106.39009 & FP, MI, S, TE & & $1983-1984$ & 2 \\
\hline 32 & Eagle River below Peterson Creek & CDPHE & EAGLE06 & 39.51722 & -106.39222 & FP, MI, TE & & 1979 & 5 \\
\hline \multirow[t]{3}{*}{34} & Eagle River above Belden Mine & USGS & 393123106234501 & 39.52304 & -106.39642 & FP, MI, S, TE & & $1983-1984$ & 2 \\
\hline & & CDPHE & 393123106234501 & 39.52288 & -106.39625 & FP, MI, TE & & 1979 & 5 \\
\hline & & DOW-RW & 3285 & & & FP, MI, TE & & 1994-2001 & 7 \\
\hline
\end{tabular}


[no., number; USGS, U.S. Geological Survey; CDPHE, Colorado Department of Public Health and Environment; CO, Colorado; WWTP, wastewater treatment plant; CSU, Colorado Springs Utilities; GRC, Grand River Consulting, EMSSA, Eagle Mine Superfund Site Assessment; DOW-RW, Division of Wildlife, River Watch; CDNR, Colorado Division of Natural Resources; ASI, Advanced Sciences Incorporated; CDOT, Colorado Department of Transportation; ERWSD, Eagle River Water and Sanitation District; USFS, U.S. Forest Service; USEPA, U.S. Environmental Protection Agency; FP, field properties; MI, major ions; TE, trace elements; O, other constituents; AE, aquatic ecology; S, sediment; N, nutrients; R, radionuclide]

\begin{tabular}{|c|c|c|c|c|c|c|c|c|c|}
\hline $\begin{array}{l}\text { Site } \\
\text { no. }\end{array}$ & Site name & $\begin{array}{c}\text { Data } \\
\text { sources }\end{array}$ & $\begin{array}{c}\text { Site } \\
\text { identifiers }\end{array}$ & Latitude & Longitude & Type of data & $\begin{array}{c}\text { Period of } \\
\text { discharge record } \\
\text { (water year) }\end{array}$ & $\begin{array}{l}\text { Period of water- } \\
\text { quality record } \\
\text { (calendar year) }\end{array}$ & $\begin{array}{c}\text { No. of } \\
\text { samples }\end{array}$ \\
\hline 35 & Eagle River, right bank 450 feet upstream E-4BR & EMSSA & E-4AR1 & 39.52522 & -106.39451 & FP, TE & & 1997 & 1 \\
\hline \multirow[t]{3}{*}{37} & Belden & USGS & 393132106233601 & 39.52554 & -106.39392 & FP, TE, MI, N & & 1970 & 1 \\
\hline & & DOW-RW & 3286 & 39.52553 & -106.39396 & FP, MI, TE & & $1992-2001$ & 14 \\
\hline & & EMSSA & E-3 & 39.52556 & -106.39389 & $\mathrm{O}, \mathrm{FP}, \mathrm{MI}, \mathrm{N}, \mathrm{TE}$ & & 1985-2003 & 147 \\
\hline 39 & Eagle River, right bank 175 feet upstream E-4BR & EMSSA & E-4AR2 & 39.52592 & -106.39424 & FP, TE & & 1997 & 1 \\
\hline \multirow[t]{2}{*}{40} & Eagle River, 25 feet upstream culvert & EMSSA & E-4BR & 39.52639 & -106.39447 & $\mathrm{FP}, \mathrm{TE}$ & & 1996-1997 & 4 \\
\hline & & EMSSA & E-4BL & & & FP, TE & & 1996 & 2 \\
\hline 41 & Eagle River above Belden Power Station & EMSSA & E-4A & 39.52660 & -106.39459 & FP, MI, N, TE & & 2001-2002 & 4 \\
\hline 42 & Eagle River, right bank 100 feet downstream E-4BR & EMSSA & E4-R1 & 39.52662 & -106.39461 & FP, TE & & 1997 & 2 \\
\hline 43 & Eagle River, right bank 200 feet downstream E-4BR & EMSSA & E4-R2 & 39.52684 & -106.39485 & FP, TE & & 1997 & 2 \\
\hline 44 & Eagle River, right bank 300 feet downstream E-4BR & EMSSA & E4-R3 & 39.52700 & -106.39519 & FP, TE & & 1997 & 2 \\
\hline 45 & Eagle River, right bank 400 feet downstream E-4BR & EMSSA & E4-R4 & 39.52715 & -106.39556 & FP, TE & & 1997 & 2 \\
\hline 46 & Eagle River, right bank 500 feet downstream E-4BR & EMSSA & E4-R5 & 39.52729 & -106.39595 & $\mathrm{FP}, \mathrm{TE}$ & & 1997 & 2 \\
\hline 47 & Eagle River, right bank 550 feet downstream E-4BR & EMSSA & E4-R5A & 39.52731 & -106.39620 & FP, TE & & 1997 & 1 \\
\hline 48 & Eagle River at Belden Power Station & EMSSA & E-4 & 39.52724 & -106.39647 & FP, TE & & 1985-1996 & 9 \\
\hline \multirow[t]{5}{*}{49} & Eagle River above Fall Creek & EMSSA & E-5 & 39.52667 & -106.39917 & $\mathrm{O}, \mathrm{FP}, \mathrm{MI}, \mathrm{N}, \mathrm{TE}$ & & 1985-2003 & 145 \\
\hline & & EMSSA & E-5D & & & $\mathrm{FP}, \mathrm{TE}$ & & 1997 & 3 \\
\hline & & EMSSA & E-5W & & & FP, TE & & 1997 & 1 \\
\hline & & EMSSA & CC-6D & & & FP, TE & & 1997 & 2 \\
\hline & & EMSSA & CC-6W & & & FP, TE & & 1997 & 4 \\
\hline \multirow[t]{2}{*}{50} & Eagle River, 40 feet upstream Fall Creek & EMSSA & E-5AR & 39.52726 & -106.40007 & FP, TE & & $1996-1997$ & 4 \\
\hline & & EMSSA & E-5AL & & & $\mathrm{FP}, \mathrm{TE}$ & & 1996 & 2 \\
\hline 51 & Fall Creek above power plant diversion & USGS & 393125106241601 & 39.52360 & -106.40503 & FP, MI, TE & & 1983 & 1 \\
\hline \multirow[t]{2}{*}{52} & Fall Creek at mouth & USGS & 393137106240001 & 39.52705 & -106.40074 & FP, MI, S, TE & & 1984 & 1 \\
\hline & & EMSSA & $\mathrm{T}-8 \mathrm{~B}$ & 39.52725 & -106.40035 & $\mathrm{FP}, \mathrm{TE}$ & & 1997 & 1 \\
\hline 54 & Eagle River, right bank at Fall Creek & EMSSA & E-5BR & 39.52732 & -106.40025 & FP, TE & & 1997 & 2 \\
\hline \multirow[t]{3}{*}{56} & Eagle River below Fall Creek & USGS & 393139106240101 & 39.52749 & -106.40062 & FP, MI, S, TE & & 1983-1984 & 2 \\
\hline & & EMSSA & E-9R & 39.52743 & -106.40031 & $\mathrm{FP}, \mathrm{TE}$ & & 1996 & 2 \\
\hline & & EMSSA & E-9L & & & FP, TE & & 1996 & 2 \\
\hline 57 & Eagle River above Rock Creek & EMSSA & E-10 & 39.53146 & -106.40050 & $\mathrm{O}, \mathrm{FP}, \mathrm{MI}, \mathrm{N}, \mathrm{TE}$ & & $1990-2003$ & 131 \\
\hline \multirow[t]{2}{*}{58} & Rock Creek at mouth & USGS & 393157106235501 & 39.53249 & -106.39920 & FP, MI, S, TE & & $1983-1984$ & 2 \\
\hline & & EMSSA & $\mathrm{T}-10$ & & & $\mathrm{O}, \mathrm{FP}, \mathrm{MI}, \mathrm{N}, \mathrm{TE}$ & & $1985-2003$ & 158 \\
\hline \multirow[t]{2}{*}{59} & Eagle River above Bishop Gulch & EMSSA & E-11 & 39.54166 & -106.40288 & $\mathrm{O}, \mathrm{FP}, \mathrm{MI}, \mathrm{N}, \mathrm{TE}$ & & 1985-2003 & 290 \\
\hline & & DOW-RW & 3287 & 39.54224 & -106.40354 & FP, MI, TE & & 1995-2001 & 10 \\
\hline \multirow[t]{2}{*}{61} & Bishop Gulch at mouth & USGS & 393233106241401 & 39.54249 & -106.40447 & FP, MI, S, TE & & 1983-1984 & 2 \\
\hline & & EMSSA & $\mathrm{T}-11$ & 39.54271 & -106.40402 & $\mathrm{FP}, \mathrm{TE}$ & & 1997 & 1 \\
\hline \multirow{3}{*}{63} & Eagle River below Bishop Gulch & USGS & 393245106241501 & 39.54582 & -106.40475 & FP, MI, S, TE & & 1983-1984 & 2 \\
\hline & & CDPHE & 393245106241501 & & & FP, TE, MI & & $1966-1968$ & 26 \\
\hline & & USEPA & 393245106241501 & & & FP, MI, N, TE & & $1966-1968$ & 4 \\
\hline
\end{tabular}


Table 5. Historic and current surface-water quantity and quality sites in the Eagle River watershed, Colorado.-Continued

[no., number; USGS, U.S. Geological Survey; CDPHE, Colorado Department of Public Health and Environment; CO, Colorado; WWTP, wastewater treatment plant; CSU, Colorado Springs Utilities; GRC, Grand River Consulting, EMSSA, Eagle Mine Superfund Site Assessment; DOW-RW, Division of Wildlife, River Watch; CDNR, Colorado Division of Natural Resources; ASI, Advanced Sciences Incorporated; CDOT, Colorado Department of Transportation; ERWSD, Eagle River Water and Sanitation District; USFS, U.S. Forest Service; USEPA, U.S. Environmental Protection Agency; FP, field properties; MI, major ions; TE, trace elements; $\mathrm{O}$, other constituents; AE, aquatic ecology; S, sediment; N, nutrients; R, radionuclide]

\begin{tabular}{|c|c|c|c|c|c|c|c|c|c|}
\hline $\begin{array}{l}\text { Site } \\
\text { no. }\end{array}$ & Site name & $\begin{array}{l}\text { Data } \\
\text { sources }\end{array}$ & $\begin{array}{c}\text { Site } \\
\text { identifiers }\end{array}$ & Latitude & Longitude & Type of data & $\begin{array}{c}\text { Period of } \\
\text { discharge record } \\
\text { (water year) }\end{array}$ & $\begin{array}{l}\text { Period of water- } \\
\text { quality record } \\
\text { (calendar year) }\end{array}$ & $\begin{array}{c}\text { No. of } \\
\text { samples }\end{array}$ \\
\hline 64 & Eagle River below Belden & CDPHE & EAGLE04 & 39.55028 & -106.40575 & FP, MI, TE & & 1979 & 5 \\
\hline \multirow[t]{6}{*}{66} & Eagle River near Minturn, CO & USGS & 09064600 & 39.55387 & -106.40253 & AE, O, FP, MI, S, N, TE & 1990-2008 & 1989-2004 & 155 \\
\hline & & EMSSA & E-12A & 39.55361 & -106.40167 & $\mathrm{O}, \mathrm{FP}, \mathrm{MI}, \mathrm{N}, \mathrm{TE}$ & & $1985-2003$ & 307 \\
\hline & & EMSSA & E-12AD & & & FP, TE & & 1997 & 3 \\
\hline & & EMSSA & E-12AW & & & FP, TE & & 1997 & 1 \\
\hline & & EMSSA & CC-42D & & & FP, TE & & 1997 & 3 \\
\hline & & EMSSA & CC-42W & & & FP, TE & & 1997 & 3 \\
\hline \multirow[t]{3}{*}{67} & Eagle River above Cross Creek near Minturn, CO & USGS & 393318106240400 & 39.55499 & -106.40170 & $\mathrm{O}, \mathrm{FP}, \mathrm{TE}, \mathrm{MI}, \mathrm{AE}, \mathrm{N}$ & & 1976-1977 & 6 \\
\hline & & CDNR & 393318106240400 & 39.55473 & -106.40142 & FP, MI, TE & & $1966-1968$ & 27 \\
\hline & & CDPHE & EAGLE03 & & & FP, MI, TE & & 1979 & 4 \\
\hline \multirow[t]{2}{*}{68} & Eagle River above Two Elk Creek & USGS & 393336106235701 & 39.55999 & -106.39975 & FP, MI, S, TE & & 1983-1984 & 2 \\
\hline & & EMSSA & E-13 & & & $\mathrm{O}, \mathrm{FP}, \mathrm{MI}, \mathrm{N}, \mathrm{TE}$ & & 1985-2003 & 52 \\
\hline 69 & Eagle River at Two Elk (3) & DOW-RW & 3289 & 39.56462 & -106.40203 & FP, MI, TE & & 1992-2000 & 15 \\
\hline \multirow[t]{2}{*}{70} & Two Elk Creek at mouth & USGS & 393353106240301 & 39.56481 & -106.40151 & FP, MI, S, TE & & 1983-1984 & 2 \\
\hline & & EMSSA & $\mathrm{T}-14$ & 39.56470 & -106.40143 & FP, TE & & 1997 & 1 \\
\hline \multirow[t]{4}{*}{72} & Eagle River above Cross Creek & USGS & 393411106242001 & 39.56986 & -106.40554 & FP, MI, S, TE & & 1984 & 1 \\
\hline & & CDNR & 393411106242001 & & & FP, TE, MI & & $1966-1968$ & 28 \\
\hline & & CSU & 393411106242001 & & & $\mathrm{O}, \mathrm{FP}, \mathrm{TE}, \mathrm{MI}, \mathrm{AE}$ & & 1987-2002 & 60 \\
\hline & & EMSSA & E-13B & & & $\mathrm{O}, \mathrm{FP}, \mathrm{MI}, \mathrm{N}, \mathrm{TE}$ & & 1985-2003 & 145 \\
\hline 73 & $\begin{array}{l}\text { Cross Creek above Battle Mountain School } \\
\text { near Minturn, CO }\end{array}$ & USGS & 393350106251200 & 39.56313 & -106.41946 & $\mathrm{O}, \mathrm{FP}, \mathrm{TE}, \mathrm{MI}, \mathrm{AE}, \mathrm{N}$ & & 1976-1977 & 6 \\
\hline 74 & Cross Creek at Maloit & CDPHE & 12563 & 39.56389 & -106.41806 & FP, MI, AE, S, N, TE & & 1996-2003 & 13 \\
\hline \multirow[t]{2}{*}{76} & Cross Creek at Minturn intake & CSU & E3_CRSSMIN & 39.56500 & -106.41639 & $\mathrm{O}, \mathrm{FP}, \mathrm{TE}, \mathrm{MI}, \mathrm{AE}, \mathrm{N}$ & & 1987-2002 & 57 \\
\hline & & DOW-RW & $98^{-}$ & 39.56474 & -106.41642 & FP, MI, TE & & 1992-1997 & 37 \\
\hline 77 & Gravel Pit: Cross Creek above Tailings Outfall & USGS & 393400106244701 & 39.56665 & -106.41364 & FP, TE, MI, N & & 1970 & 1 \\
\hline \multirow[t]{2}{*}{78} & Cross Creek near Minturn, $\mathrm{CO}$ & USGS & 09065100 & 39.56805 & -106.41202 & $\mathrm{AE}, \mathrm{O}, \mathrm{FP}, \mathrm{TE}, \mathrm{MI}, \mathrm{S}, \mathrm{N}$ & $1957-1963,1967-2008$ & 1960-2004 & 411 \\
\hline & & CDPHE & 09065100 & & & FP, TE, R, MI, AE, S, N, O & & 1971-2001 & 192 \\
\hline \multirow[t]{4}{*}{79} & Cross Creek near mouth & EMSSA & $\mathrm{T}-18$ & 39.56972 & -106.40778 & $\mathrm{O}, \mathrm{FP}, \mathrm{MI}, \mathrm{N}, \mathrm{TE}$ & & $1985-2003$ & 134 \\
\hline & & CDNR & MINT03 & 39.57015 & -106.40701 & FP, MI, TE & & $1966-1968$ & 28 \\
\hline & & USGS & 393413106242301 & 39.57026 & -106.40697 & FP, MI, S, TE & & 1983-1984 & 2 \\
\hline & & USGS & 393413106242300 & & & FP, MI, TE & & 1971 & 1 \\
\hline 82 & Eagle River below Cross Creek & DOW-RW & 3288 & 39.57093 & -106.40597 & FP, TE & & 1992 & 1 \\
\hline \multirow[t]{3}{*}{83} & Eagle River below Cross Creek & USGS & 393422106242001 & 39.57276 & -106.40614 & FP, TE, MI, N & & 1970 & 1 \\
\hline & & CDNR & 393422106242001 & & & FP, MI, TE & & $1966-1968$ & 17 \\
\hline & & USEPA & 393422106242001 & & & FP, MI, N, TE & & $1966-1968$ & 4 \\
\hline \multirow[t]{3}{*}{84} & Eagle River below Cross Creek & EMSSA & E-14 & 39.57472 & -106.40694 & $\mathrm{O}, \mathrm{FP}, \mathrm{MI}, \mathrm{N}, \mathrm{TE}$ & & 1985-1997 & 107 \\
\hline & & CSU & E1_BCRSS & 39.57472 & -106.40750 & $\mathrm{FP}, \mathrm{N}$ & & 1987 & 2 \\
\hline & & DOW-RW & 3290 & 39.57493 & -106.40850 & FP, MI, TE & & 1993-2001 & 12 \\
\hline \multirow[t]{2}{*}{88} & Eagle River below Cross Creek & EMSSA & E-15 & 39.57694 & -106.41139 & $\mathrm{O}, \mathrm{FP}, \mathrm{MI}, \mathrm{N}, \mathrm{TE}$ & & $1992-2003$ & 246 \\
\hline & & DOW-RW & 3291 & 39.57606 & -106.41015 & $\mathrm{TE}$ & & 1994 & 1 \\
\hline
\end{tabular}


[no., number; USGS, U.S. Geological Survey; CDPHE, Colorado Department of Public Health and Environment; CO, Colorado; WWTP, wastewater treatment plant; CSU, Colorado Springs Utilities; GRC, Grand River Consulting, EMSSA, Eagle Mine Superfund Site Assessment; DOW-RW, Division of Wildlife, River Watch; CDNR, Colorado Division of Natural Resources; ASI, Advanced Sciences Incorporated; CDOT, Colorado Department of Transportation; ERWSD, Eagle River Water and Sanitation District; USFS, U.S. Forest Service; USEPA, U.S. Environmental Protection Agency; FP, field properties; MI, major ions; TE, trace elements; O, other constituents; AE, aquatic ecology; S, sediment; N, nutrients; R, radionuclide]

\begin{tabular}{|c|c|c|c|c|c|c|c|c|c|}
\hline $\begin{array}{l}\text { Site } \\
\text { no. }\end{array}$ & Site name & $\begin{array}{l}\text { Data } \\
\text { sources }\end{array}$ & $\begin{array}{c}\text { Site } \\
\text { identifiers }\end{array}$ & Latitude & Longitude & Type of data & $\begin{array}{c}\text { Period of } \\
\text { discharge record } \\
\text { (water year) }\end{array}$ & $\begin{array}{l}\text { Period of water- } \\
\text { quality record } \\
\text { (calendar year) }\end{array}$ & $\begin{array}{c}\text { No. of } \\
\text { samples }\end{array}$ \\
\hline 89 & Unnamed tributary Eagle River above Minturn & USGS & 393443106245201 & 39.57860 & -106.41503 & FP, MI, S, TE & & 1984 & 1 \\
\hline \multirow[t]{3}{*}{90} & Eagle River above Minturn, $\mathrm{CO}$ & USGS & 393454106252201 & 39.58165 & -106.42336 & FP, MI, S, TE & & 1983-1984 & 2 \\
\hline & & CSU & E5_MIN & 39.58174 & -106.42332 & $\mathrm{O}, \mathrm{FP}, \mathrm{TE}, \mathrm{MI}, \mathrm{AE}$ & & $1987-2002$ & 60 \\
\hline & & EMSSA & E5_MIN & & & FP, MI, TE & & 1985-1992 & 16 \\
\hline 92 & Martin Creek at mouth & USGS & 393454106252301 & 39.58165 & -106.42364 & FP, MI, S, TE & & 1983-1984 & 2 \\
\hline 93 & Eagle River at county bridge & DOW-RW & 39 & 39.58190 & -106.42358 & FP, MI, TE & & 1991 & 7 \\
\hline \multirow[t]{2}{*}{94} & Eagle River at Minturn & CDPHE & E-21 & 39.58940 & -106.43139 & FP, MI, AE, N, TE & & 1996-1997 & 9 \\
\hline & & EMSSA & E-21 & & & FP, MI, TE & & 1991 & 1 \\
\hline 95 & Grouse Creek at mouth & USGS & 393543106260001 & 39.59526 & -106.43392 & FP, MI, S, TE & & 1983-1984 & 2 \\
\hline 96 & Game Creek at mouth & USGS & 393548106255201 & 39.59665 & -106.43170 & FP, MI, S, TE & & 1983-1984 & 2 \\
\hline \multirow[t]{2}{*}{97} & Eagle River below Minturn, $\mathrm{CO}$ & USGS & 393550106262001 & 39.59744 & -106.43356 & FP, TE & & 1979 & 1 \\
\hline & & EMSSA & E-22 & 39.59778 & -106.43361 & $\mathrm{O}, \mathrm{FP}, \mathrm{MI}, \mathrm{N}, \mathrm{TE}$ & & 1985-2001 & 32 \\
\hline \multirow[t]{4}{*}{100} & Eagle River above Gore Creek near Minturn, CO & USGS & 393627106264000 & 39.60749 & -106.44503 & AE, O, FP, MI, S, N, TE & & $1995-2003$ & 15 \\
\hline & & ASI & 393627106264000 & 39.60789 & -106.44657 & FP, MI, TE & & $1988-1990$ & 33 \\
\hline & & CDPHE & $12503 \mathrm{G}$ & 39.60582 & -106.44192 & FP, TE, MI, AE, S, N & & $1997-2005$ & 29 \\
\hline & & DOW-RW & 3292 & & & FP, MI, TE & & 1991-2001 & 74 \\
\hline \multirow[t]{2}{*}{102} & Gore Creek at Upper Station, near Minturn, CO & USGS & 09065500 & 39.62582 & -106.27808 & AE, O, FP, TE, MI, S, N & 1948-1956, 1964-2008 & $1963-2004$ & 417 \\
\hline & & ASI & 09065500 & & & FP, MI, TE & & 1988-1989 & 12 \\
\hline \multirow[t]{2}{*}{103} & Black Gore Creek above Black Lake & USGS & 393212106125800 & 39.53665 & -106.21669 & $\mathrm{O}, \mathrm{FP}, \mathrm{MI}, \mathrm{S}, \mathrm{N}, \mathrm{TE}$ & & 1996 & 1 \\
\hline & & ASI & 393212106125800 & & & FP, MI, TE & & 1988-1989 & 6 \\
\hline \multirow[t]{2}{*}{105} & Black Gore Creek below Black Lake No. 2 & USGS & 393307106133200 & 39.55193 & -106.22614 & $\mathrm{O}, \mathrm{FP}, \mathrm{MI}, \mathrm{S}, \mathrm{N}, \mathrm{TE}$ & & 1996 & 1 \\
\hline & & ASI & 393307106133200 & & & FP, MI, TE & & 1988-1989 & 13 \\
\hline 106 & Polk Creek above US6 & CDOT & PC-2 & 39.59018 & -106.24166 & FP, MI, N, TE & & $2001-2003$ & 18 \\
\hline 107 & Polk Creek at Interstate 70 & USGS & 393527106143500 & 39.59082 & -106.24364 & $\mathrm{O}, \mathrm{FP}, \mathrm{MI}, \mathrm{S}, \mathrm{N}, \mathrm{TE}$ & & $1996-1999$ & 7 \\
\hline 108 & Miller Creek near mouth & CDOT & UN-1 & 39.59556 & -106.25167 & FP, MI, N, TE & & 2001 & 1 \\
\hline \multirow[t]{2}{*}{110} & Black Gore Creek near Minturn, CO & USGS & 09066000 & 39.59637 & -106.26503 & $\mathrm{AE}, \mathrm{O}, \mathrm{FP}, \mathrm{MI}, \mathrm{S}, \mathrm{N}, \mathrm{TE}$ & 1948-1956, 1964-2008 & $1963-2003$ & 411 \\
\hline & & CDOT & BG-2 & 39.59641 & -106.26421 & FP, MI, N, TE & & $2000-2003$ & 99 \\
\hline \multirow[t]{2}{*}{111} & Black Gore Creek near Vail, CO & USGS & 09066050 & 39.62332 & -106.28030 & AE, O, FP, MI, S, N, TE & 1974-1979 & 1973-2001 & 99 \\
\hline & & ASI & 09066050 & & & FP, MI, TE & & 1988-1990 & 23 \\
\hline 112 & Gore Creek below Black Gore Creek near Vail, CO & USGS & 393737106165900 & 39.62693 & -106.28364 & $\mathrm{O}, \mathrm{FP}, \mathrm{TE}, \mathrm{R}, \mathrm{MI}, \mathrm{AE}, \mathrm{S}, \mathrm{N}$ & & 1996-2007 & 6 \\
\hline 113 & Gore Creek above Bighorn Creek near Vail, CO & USGS & 393807106174600 & 39.63526 & -106.29669 & $\mathrm{O}, \mathrm{FP}, \mathrm{MI}, \mathrm{S}, \mathrm{N}, \mathrm{TE}$ & & 1996 & 1 \\
\hline 114 & Bighorn Creek near Minturn, CO & USGS & 09066100 & 39.63999 & -106.29336 & $\mathrm{O}, \mathrm{FP}, \mathrm{MI}, \mathrm{S}, \mathrm{N}, \mathrm{TE}$ & 1964-2008 & $1963-2003$ & 390 \\
\hline 115 & Bighorn Creek near Vail, CO & USGS & 393813106174500 & 39.63792 & -106.29687 & $\mathrm{AE}$ & & 1997 & 2 \\
\hline 116 & Pitkin Creek near Minturn, $\mathrm{CO}$ & USGS & 09066150 & 39.64360 & -106.30253 & $\mathrm{O}, \mathrm{FP}, \mathrm{MI}, \mathrm{S}, \mathrm{N}, \mathrm{TE}$ & 1967-2008 & $1965-2003$ & 381 \\
\hline \multirow[t]{4}{*}{117} & Gore Creek at Bighorn Subdivision below & USGS & 393831106181900 & 39.64193 & -106.30586 & $\mathrm{O}, \mathrm{FP}, \mathrm{MI}, \mathrm{S}, \mathrm{N}, \mathrm{TE}$ & & 1996-1999 & 3 \\
\hline & Pitkin Creek & ASI & 393831106181900 & 39.64332 & -106.30753 & FP, MI, TE & & 1988-1989 & 27 \\
\hline & & CDPHE & 393831106181900 & & & FP, TE, R, MI, AE, S, N, O & & $1968-2001$ & 164 \\
\hline & & USGS & 393836106182500 & & & $\mathrm{AE}, \mathrm{FP}$ & & 1997-1999 & 3 \\
\hline 119 & Gore Creek below Katsos & USGS & 393848106185900 & 39.64600 & -106.31699 & $\mathrm{AE}$ & & 1997 & 1 \\
\hline
\end{tabular}


Table 5. Historic and current surface-water quantity and quality sites in the Eagle River watershed, Colorado.-Continued

[no., number; USGS, U.S. Geological Survey; CDPHE, Colorado Department of Public Health and Environment; CO, Colorado; WWTP, wastewater treatment plant; CSU, Colorado Springs Utilities; GRC, Grand River Consulting, EMSSA, Eagle Mine Superfund Site Assessment; DOW-RW, Division of Wildlife, River Watch; CDNR, Colorado Division of Natural Resources; ASI, Advanced Sciences Incorporated; CDOT, Colorado Department of Transportation; ERWSD, Eagle River Water and Sanitation District; USFS, U.S. Forest Service; USEPA, U.S. Environmental Protection Agency; FP, field properties; MI, major ions; TE, trace elements; $\mathrm{O}$, other constituents; AE, aquatic ecology; S, sediment; N, nutrients; R, radionuclide]

\begin{tabular}{|c|c|c|c|c|c|c|c|c|c|}
\hline $\begin{array}{l}\text { Site } \\
\text { no. }\end{array}$ & Site name & $\begin{array}{c}\text { Data } \\
\text { sources }\end{array}$ & $\begin{array}{c}\text { Site } \\
\text { identifiers }\end{array}$ & Latitude & Longitude & Type of data & $\begin{array}{c}\text { Period of } \\
\text { discharge record } \\
\text { (water year) }\end{array}$ & $\begin{array}{l}\text { Period of water- } \\
\text { quality record } \\
\text { (calendar year) }\end{array}$ & $\begin{array}{c}\text { No. of } \\
\text { samples }\end{array}$ \\
\hline \multirow[t]{2}{*}{120} & Booth Creek near Minturn, CO & USGS & 09066200 & 39.64832 & -106.32300 & $\mathrm{AE}, \mathrm{O}, \mathrm{FP}, \mathrm{MI}, \mathrm{S}, \mathrm{N}, \mathrm{TE}$ & 1965-2008 & $1964-2003$ & 404 \\
\hline & & USGS & 393849106192000 & 39.64693 & -106.32281 & $\mathrm{AE}$ & & 1997 & 2 \\
\hline \multirow[t]{2}{*}{122} & Gore Creek above well field, near Vail, CO & USGS & 393844106192100 & 39.64554 & -106.32308 & $\mathrm{O}, \mathrm{FP}, \mathrm{MI}, \mathrm{S}, \mathrm{N}, \mathrm{TE}$ & & 1996 & 1 \\
\hline & & ASI & 393844106192100 & & & FP, MI, TE & & $1988-1990$ & 33 \\
\hline 123 & Gore Creek at Booth Creek Road & USGS & 393851106193100 & 39.64554 & -106.32577 & $\mathrm{AE}, \mathrm{FP}$ & & 1997-1999 & 3 \\
\hline 124 & Gore Creek at Golf Course at Vail, CO & USGS & 393844106195300 & 39.64533 & -106.33204 & $\mathrm{FP}, \mathrm{MI}, \mathrm{N}, \mathrm{TE}$ & & 1995 & 1 \\
\hline \multirow[t]{2}{*}{125} & Gore Creek at Vail, $\mathrm{CO}$ & USGS & 09066250 & 39.64242 & -106.34589 & $\mathrm{O}, \mathrm{FP}, \mathrm{MI}, \mathrm{S}, \mathrm{N}, \mathrm{TE}$ & 1974-1979 & 1973-1996 & 101 \\
\hline & & ASI & 09066250 & & & $\mathrm{FP}, \mathrm{MI}, \mathrm{TE}$ & & $1988-1990$ & 12 \\
\hline 126 & Gore Creek at Vail WWTP Intake & USGS & 393826106212900 & 39.64036 & -106.35864 & $\mathrm{O}, \mathrm{FP}, \mathrm{TE}, \mathrm{MI}, \mathrm{AE}, \mathrm{S}, \mathrm{N}$ & & $1976-1977$ & 5 \\
\hline 127 & Gore Creek downstream of Pulis Bridge & USGS & 393825106213400 & 39.64026 & -106.36003 & $\mathrm{AE}, \mathrm{FP}$ & & 1997-1999 & 4 \\
\hline 128 & Gore Creek at bike path bridge & DOW-RW & 526 & 39.63876 & -106.36458 & FP, MI, TE & & $1995-2001$ & 83 \\
\hline 129 & Gore Creek below golf course, at Vail, $\mathrm{CO}$ & USGS & 393825106220000 & 39.64026 & -106.36725 & $\mathrm{O}, \mathrm{FP}, \mathrm{MI}, \mathrm{S}, \mathrm{N}, \mathrm{TE}$ & & 1996 & 1 \\
\hline 130 & Mill Creek on Vail Mountain & USGS & 393726106212000 & 39.62527 & -106.35617 & $\mathrm{AE}$ & & 1997 & 1 \\
\hline \multirow[t]{2}{*}{131} & Mill Creek at ski area at Vail, $\mathrm{CO}$ & USGS & 393814106221500 & 39.63721 & -106.37099 & $\mathrm{O}, \mathrm{FP}, \mathrm{MI}, \mathrm{S}, \mathrm{N}, \mathrm{TE}$ & & 1996 & 1 \\
\hline & & ASI & 393814106221500 & & & $\mathrm{FP}, \mathrm{MI}, \mathrm{TE}$ & & $1988-1990$ & 14 \\
\hline \multirow[t]{2}{*}{132} & Mill Creek near Vail, CO & USGS & 393824106221700 & 39.63991 & -106.37211 & $\mathrm{AE}, \mathrm{O}, \mathrm{FP}, \mathrm{MI}, \mathrm{S}, \mathrm{N}, \mathrm{TE}$ & & $1997-2001$ & 9 \\
\hline & & USGS & 393827106222100 & 39.64075 & -106.37266 & $\mathrm{O}, \mathrm{FP}, \mathrm{TE}, \mathrm{MI}, \mathrm{AE}, \mathrm{N}$ & & $1976-1977$ & 2 \\
\hline 134 & Gore Creek at Middle Creek & USGS & 393826106223800 & 39.64104 & -106.37782 & $\mathrm{AE}, \mathrm{FP}$ & & 1997-1999 & 3 \\
\hline 135 & Middle Creek near Minturn, CO & USGS & 09066300 & 39.64571 & -106.38189 & $\mathrm{O}, \mathrm{FP}, \mathrm{MI}, \mathrm{S}, \mathrm{N}, \mathrm{TE}$ & 1965-2008 & $1964-2003$ & 373 \\
\hline 136 & Middle Creek near Vail, CO & USGS & 393836106230100 & 39.64320 & -106.38391 & $\mathrm{AE}$ & & 1997 & 2 \\
\hline 137 & Gore Creek above STP near Vail, CO & USGS & 393901106231400 & 39.64167 & -106.38893 & $\mathrm{O}, \mathrm{FP}, \mathrm{TE}, \mathrm{MI}, \mathrm{AE}, \mathrm{N}$ & & 1976-1977 & 5 \\
\hline \multirow[t]{5}{*}{139} & Gore Creek above Red Sandstone Creek at Vail, CO & USGS & 09066325 & 39.64100 & -106.39473 & FP & $2000-2008$ & $1999-2003$ & 41 \\
\hline & & USGS & 09066310 & 39.64110 & -106.39420 & $\mathrm{AE}, \mathrm{O}, \mathrm{FP}, \mathrm{MI}, \mathrm{S}, \mathrm{N}, \mathrm{TE}$ & $1988-1999$ & $1988-2000$ & 124 \\
\hline & & ERWSD & 09066325 & & & FP, TE, MI, AE, N & & $1990-2005$ & 264 \\
\hline & & ASI & 09066310 & & & FP, MI, N, TE & & $1988-1990$ & 33 \\
\hline & & CDPHE & 09066310 & & & $\mathrm{FP}, \mathrm{MI}, \mathrm{AE}, \mathrm{N}, \mathrm{TE}$ & & $1996-1997$ & 11 \\
\hline \multirow[t]{2}{*}{140} & Gore Creek below Vail WWTP outfall at Vail, CO & USGS & 393829106234000 & 39.64096 & -106.39493 & $\mathrm{FP}$ & & 1999 & 2 \\
\hline & & ERWSD & 393829106234000 & & & FP, TE, MI, AE, N & & $1990-2005$ & 141 \\
\hline 141 & Red Sandstone Creek near Minturn, CO & USGS & 09066400 & 39.68276 & -106.40142 & $\mathrm{FP}$ & $1964-2008$ & $1963-2004$ & 344 \\
\hline 142 & Red Sandstone Creek near Vail, CO & USGS & 393852106234300 & 39.64776 & -106.39619 & $\mathrm{AE}$ & & 1997 & 1 \\
\hline 143 & Red Sandstone Creek near Vail, CO & USGS & 393841106234200 & 39.64470 & -106.39594 & $\mathrm{AE}$ & & 1997 & 2 \\
\hline 144 & Red Sandstone Creek at mouth at Vail, CO & USGS & 393829106234400 & 39.64132 & -106.39571 & $\mathrm{O}, \mathrm{FP}, \mathrm{MI}, \mathrm{S}, \mathrm{N}, \mathrm{TE}$ & & $1996-1999$ & 3 \\
\hline 145 & Gore Creek below WWTP & USGS & 393826106235300 & 39.64018 & -106.39852 & $\mathrm{AE}, \mathrm{FP}$ & & $1997-2000$ & 3 \\
\hline \multirow[t]{2}{*}{146} & Gore Creek below Red Sandstone Creek at Vail, CO & USGS & 393823106240000 & 39.63947 & -106.40002 & $\mathrm{O}, \mathrm{FP}, \mathrm{MI}, \mathrm{S}, \mathrm{N}, \mathrm{TE}$ & & 1996 & 1 \\
\hline & & ASI & 393823106240000 & & & FP, MI, N, TE & & $1988-1989$ & 27 \\
\hline 148 & Gore Creek 0.5 miles downstream of WWTP & ERWSD & GC1/2MDNSTP & 39.63750 & -106.40130 & FP & & $1994-1996$ & 10 \\
\hline 149 & Gore Creek 1 mile downstream of WWTP & ERWSD & GC1MDNSTP & 39.63361 & -106.40917 & FP & & 1994-1996 & 10 \\
\hline 150 & Buffehr Creek near Vail, CO & USGS & 393801106244800 & 39.63360 & -106.41392 & $\mathrm{AE}, \mathrm{FP}$ & & $1997-1999$ & 4 \\
\hline 151 & Gore Creek below Buffehr Creek near West Vail, CO & USGS & 393756106244300 & 39.63185 & -106.41263 & $\mathrm{O}, \mathrm{FP}, \mathrm{MI}, \mathrm{S}, \mathrm{N}, \mathrm{TE}$ & & 1996 & 1 \\
\hline
\end{tabular}


Table 5. Historic and current surface-water quantity and quality sites in the Eagle River watershed, Colorado.-Continued

[no., number; USGS, U.S. Geological Survey; CDPHE, Colorado Department of Public Health and Environment; CO, Colorado; WWTP, wastewater treatment plant; CSU, Colorado Springs Utilities; GRC, Grand River Consulting, EMSSA, Eagle Mine Superfund Site Assessment; DOW-RW, Division of Wildlife, River Watch; CDNR, Colorado Division of Natural Resources; ASI, Advanced Sciences Incorporated; CDOT, Colorado Department of Transportation; ERWSD, Eagle River Water and Sanitation District; USFS, U.S. Forest Service; USEPA, U.S. Environmental Protection Agency; FP, field properties; MI, major ions; TE, trace elements; O, other constituents; AE, aquatic ecology; S, sediment; N, nutrients; R, radionuclide]

\begin{tabular}{|c|c|c|c|c|c|c|c|c|c|}
\hline $\begin{array}{l}\text { Site } \\
\text { no. }\end{array}$ & Site name & $\begin{array}{l}\text { Data } \\
\text { sources }\end{array}$ & $\begin{array}{c}\text { Site } \\
\text { identifiers }\end{array}$ & Latitude & Longitude & Type of data & $\begin{array}{c}\text { Period of } \\
\text { discharge record } \\
\text { (water year) }\end{array}$ & $\begin{array}{l}\text { Period of water- } \\
\text { quality record } \\
\text { (calendar year) }\end{array}$ & $\begin{array}{c}\text { No. of } \\
\text { samples }\end{array}$ \\
\hline 152 & Gore Creek 1.5 miles downstream of WWTP & ERWSD & GC15MDNSTP & 39.62944 & -106.41472 & FP & & 1994-1996 & 10 \\
\hline 153 & Gore Creek at West Vail exit & USGS & 393738106251000 & 39.62721 & -106.42003 & $\mathrm{O}, \mathrm{FP}, \mathrm{MI}, \mathrm{S}, \mathrm{N}, \mathrm{TE}$ & & 1996 & 1 \\
\hline 154 & Gore Creek 2 miles downstream of WWTP & ERWSD & GC2MDNSTP & 39.62556 & -106.42194 & FP & & 1994-1996 & 9 \\
\hline 155 & Gore Creek at Stephens Park & USGS & 393715106253600 & 39.62082 & -106.42725 & $\mathrm{AE}, \mathrm{FP}$ & & 1997-1999 & 5 \\
\hline 156 & Gore Creek at West Vail, CO & USGS & 393713106253900 & 39.62026 & -106.42809 & O, FP, MI, S, N, TE & & 1995-1996 & 2 \\
\hline 157 & Gore Creek at mouth & CDPHE & 000074 & 39.61675 & -106.43608 & FP, TE, R, MI, AE, S, N & & 1968-2005 & 181 \\
\hline \multirow[t]{2}{*}{160} & Gore Creek at mouth, near Minturn, $\mathrm{CO}$ & USGS & 09066510 & 39.60943 & -106.44781 & $\mathrm{AE}, \mathrm{O}, \mathrm{FP}, \mathrm{TE}, \mathrm{R}, \mathrm{MI}, \mathrm{S}, \mathrm{N}$ & 1996-2008 & 1995-2007 & 264 \\
\hline & & ASI & 09066510 & & & FP, MI, TE & & 1988-1990 & 33 \\
\hline \multirow[t]{2}{*}{161} & Eagle River below Gore Creek & USGS & 393638106271401 & 39.61054 & -106.45447 & FP, MI, S, TE & & 1983-1984 & 2 \\
\hline & & ASI & 393638106271401 & & & FP, MI, TE & & $1988-1990$ & 33 \\
\hline \multirow[t]{2}{*}{163} & Eagle River below Gore Creek below Eagle-Vail & USGS & 393711106275200 & 39.61971 & -106.46503 & O, FP, TE, MI, AE, S, N & & 1976-1977 & 4 \\
\hline & Intake at Dowds Junction & DOW-RW & 151 & 39.61935 & -106.46546 & FP, MI, TE & & $2000-2004$ & 40 \\
\hline 164 & SC00508117BCA Eagle-Vail & USGS & 393708106291701 & 39.61888 & -106.48864 & FP, MI, N, TE & & 1976 & 1 \\
\hline 165 & Beaver Creek downstream Beaver Lake & CDPHE & $12542 \mathrm{D}$ & 39.56536 & -106.53480 & FP, MI, AE, S, N, TE & & 2001 & 1 \\
\hline 166 & Beaver Creek above Avon, CO & USGS & 393501106313200 & 39.58361 & -106.52556 & AE, O, FP, MI, S, N, TE & & 2000-2001 & 3 \\
\hline 167 & Beaver Creek above Town & USGS & 393552106311800 & 39.59776 & -106.52225 & $\mathrm{AE}$ & & 1997 & 3 \\
\hline 168 & Beaver Creek at top of Golf Course at Avon, $\mathrm{CO}$ & USGS & 393633106311000 & 39.60917 & -106.51853 & $\mathrm{AE}$ & & 1997 & 2 \\
\hline 169 & Beaver Creek at Avon, $\mathrm{CO}$ & USGS & 09067000 & 39.62971 & -106.52281 & AE, O, FP, MI, S, N, TE & $1974-2008$ & 1975-2004 & 228 \\
\hline \multirow[t]{3}{*}{171} & Beaver Creek near mouth & USGS & 393752106312700 & 39.63119 & -106.52167 & $\mathrm{AE}$ & & 1997 & 3 \\
\hline & & CDPHE & 000143 & 39.63111 & -106.52139 & FP, TE, MI, AE, S, N & & 1969-1999 & 106 \\
\hline & & CDPHE & 12541 & & & FP, MI, AE, S, N, TE & & 2001-2002 & 6 \\
\hline 172 & Eagle River at Avon & DOW-RW & 41 & 39.63187 & -106.52228 & FP, MI, TE & & 1991-1993 & 36 \\
\hline \multirow[t]{5}{*}{173} & Eagle River at Avon, CO & USGS & 09067005 & 39.63212 & -106.52422 & $\mathrm{AE}, \mathrm{O}, \mathrm{FP}, \mathrm{R}, \mathrm{MI}, \mathrm{S}, \mathrm{N}, \mathrm{TE}$ & 1989-2000 & 1977-2007 & 251 \\
\hline & & CDPHE & 09067005 & 39.63194 & -106.52500 & FP, TE, MI, AE, N & & 1997-1998 & 8 \\
\hline & & CSU & 09067005 & & & $\mathrm{O}, \mathrm{FP}, \mathrm{TE}, \mathrm{MI}, \mathrm{AE}$ & & $1987-2002$ & 60 \\
\hline & & ERWSD & 09067005 & & & FP, TE, MI, AE, N & & 1990-2005 & 136 \\
\hline & & CDPHE & 12503D & & & FP, TE, MI, AE, S, N & & 1997-1999 & 10 \\
\hline 175 & Eagle River at Edwards & CDPHE & $12502 \mathrm{E}$ & 39.63334 & -106.52500 & FP, TE, MI, AE, S, N & & 1997-2002 & 23 \\
\hline \multirow[t]{2}{*}{176} & Eagle River below WWTP at Avon, CO & USGS & 09067020 & 39.63500 & -106.53250 & FP & 2000-2008 & 1999-2001 & 7 \\
\hline & & ERWSD & 09067020 & & & FP, TE, MI, AE, N & & $1990-2005$ & 139 \\
\hline 177 & June Creek & USGS & 394005106330001 & 39.66764 & -106.55298 & FP, MI, N, TE & & 1979 & 1 \\
\hline \multirow[t]{2}{*}{178} & Eagle River below Beaver Creek & EMSSA & E-28 & 39.63990 & -106.56218 & $\mathrm{O}, \mathrm{FP}, \mathrm{MI}, \mathrm{N}, \mathrm{TE}$ & & 1995-2001 & 18 \\
\hline & & DOW-RW & 3293 & 39.64006 & -106.56226 & FP, MI, TE & & $1992-2001$ & 10 \\
\hline \multirow[t]{2}{*}{180} & Eagle River at Edwards, CO & USGS & 393845106353000 & 39.64582 & -106.59226 & $\mathrm{AE}, \mathrm{O}, \mathrm{FP}, \mathrm{MI}, \mathrm{S}, \mathrm{N}, \mathrm{TE}$ & & 1995-2001 & 9 \\
\hline & & CDPHE & 393845106353000 & & & FP, TE, R, MI, AE, S, N, O & & 1970-1997 & 338 \\
\hline 181 & Berry Creek & USGS & 393940106345501 & 39.66098 & -106.58173 & FP, MI, N, TE & & 1979 & 1 \\
\hline 182 & West Lake Creek near Edwards, CO & USGS & 393523106364700 & 39.58972 & -106.61306 & $\mathrm{AE}, \mathrm{O}, \mathrm{FP}, \mathrm{MI}, \mathrm{S}, \mathrm{N}, \mathrm{TE}$ & & 2000 & 2 \\
\hline 183 & East Lake Creek & USGS & 393720106370001 & 39.62211 & -106.61607 & FP, TE & & 1979 & 1 \\
\hline
\end{tabular}


Table 5. Historic and current surface-water quantity and quality sites in the Eagle River watershed, Colorado.-Continued

[no., number; USGS, U.S. Geological Survey; CDPHE, Colorado Department of Public Health and Environment; CO, Colorado; WWTP, wastewater treatment plant; CSU, Colorado Springs Utilities; GRC, Grand River Consulting, EMSSA, Eagle Mine Superfund Site Assessment; DOW-RW, Division of Wildlife, River Watch; CDNR, Colorado Division of Natural Resources; ASI, Advanced Sciences Incorporated; CDOT, Colorado Department of Transportation; ERWSD, Eagle River Water and Sanitation District; USFS, U.S. Forest Service; USEPA, U.S. Environmental Protection Agency; FP, field properties; MI, major ions; TE, trace elements; $\mathrm{O}$, other constituents; AE, aquatic ecology; S, sediment; N, nutrients; R, radionuclide]

\begin{tabular}{|c|c|c|c|c|c|c|c|c|c|}
\hline $\begin{array}{l}\text { Site } \\
\text { no. }\end{array}$ & Site name & $\begin{array}{l}\text { Data } \\
\text { sources }\end{array}$ & $\begin{array}{c}\text { Site } \\
\text { identifiers }\end{array}$ & Latitude & Longitude & Type of data & $\begin{array}{c}\text { Period of } \\
\text { discharge record } \\
\text { (water year) }\end{array}$ & $\begin{array}{l}\text { Period of water- } \\
\text { quality record } \\
\text { (calendar year) }\end{array}$ & $\begin{array}{c}\text { No. of } \\
\text { samples }\end{array}$ \\
\hline \multirow[t]{2}{*}{184} & Lake Creek near Edwards, $\mathrm{CO}$ & USGS & 09067200 & 39.64749 & -106.60920 & $\mathrm{AE}, \mathrm{O}, \mathrm{FP}, \mathrm{MI}, \mathrm{S}, \mathrm{N}, \mathrm{TE}$ & 1994-2008 & 1993-2004 & 94 \\
\hline & & CDPHE & $12502 \mathrm{D}$ & 39.64807 & -106.60955 & FP, MI, AE, S, N, TE & & 1999-2002 & 3 \\
\hline 186 & Eagle River above Edwards WWTP & DOW-RW & 3294 & 39.65303 & -106.62098 & $\mathrm{TE}$ & & 1995 & 1 \\
\hline $187 \mathrm{~A}$ & Eagle River 50 feet upstream Squaw WWTP & ERWSD & $\begin{array}{l}\text { ER50FTUPSW- } \\
\text { WTP }\end{array}$ & 39.65250 & -106.62361 & FP, TE, MI, AE, N & & $1990-2005$ & 139 \\
\hline 187B & Eagle River 50 feet downstream Squaw WWTP & ERWSD & $\begin{array}{l}\text { ER50FTDNSW- } \\
\text { WTP }\end{array}$ & 39.65250 & -106.62361 & FP, TE, MI, AE, N & & $1990-2005$ & 139 \\
\hline 188 & Eagle River below Edwards WWTP & DOW-RW & 3295 & 39.65419 & -106.62772 & FP, MI, TE & & 1994-1997 & 2 \\
\hline \multirow[t]{2}{*}{189} & Squaw Creek & USGS & 393930106382001 & 39.65836 & -106.63819 & AE, O, FP, TE, MI, S, N & & 1979-2000 & 3 \\
\hline & & CDPHE & $12502 \mathrm{C}$ & 39.65838 & -106.63819 & FP, MI, AE, S, N & & 1999 & 1 \\
\hline 190 & Eagle River at Eagle Springs golf course & CDPHE & $12502 \mathrm{~A}$ & 39.69056 & -106.66000 & FP, TE, MI, AE, S, N & & 1997-2001 & 26 \\
\hline \multirow[t]{2}{*}{191} & Eagle River at Eagle Springs golf course near & USGS & 394129106393300 & 39.69138 & -106.65976 & AE, O, FP, MI, S, N, TE & & $1997-2000$ & 8 \\
\hline & Wolcott, CO & CDPHE & 394129106393300 & & & FP, TE, MI, AE, N & & $1997-1998$ & 10 \\
\hline \multirow[t]{2}{*}{193} & Eagle River at Wolcott, $\mathrm{CO}$ & CDPHE & 12502 & 39.70139 & -106.67833 & FP, MI, AE, N, TE & & 1996-1997 & 11 \\
\hline & & DOW-RW & 3296 & 39.70144 & -106.67805 & FP, MI, TE & & 1992-1997 & 4 \\
\hline 194 & Alkali Creek near Wolcott, CO & USGS & 09067300 & 39.75610 & -106.67059 & FP & 1959-1965 & $1960-1965$ & 43 \\
\hline 195 & Alkali Creek near Wolcott & CDPHE & 12575 & 39.72948 & -106.67875 & FP, MI, AE, S, N, TE & & 2001-2005 & 5 \\
\hline 196 & Alkali Creek below Muddy Creek, near Wolcott, CO & USGS & 394259106405900 & 39.71639 & -106.68306 & $\mathrm{O}, \mathrm{FP}, \mathrm{MI}, \mathrm{AE}, \mathrm{S}, \mathrm{N}, \mathrm{TE}$ & & 1979-2007 & 19 \\
\hline 197 & Milk Creek 2 miles above mouth & USGS & 394415106424200 & 39.73750 & -106.71167 & $\mathrm{O}, \mathrm{FP}, \mathrm{MI}, \mathrm{S}, \mathrm{N}, \mathrm{TE}$ & & 2000 & 1 \\
\hline 198 & Milk Creek at mouth near Wolcott, $\mathrm{CO}$ & USGS & 394240106433200 & 39.71164 & -106.70942 & $\mathrm{O}, \mathrm{FP}, \mathrm{MI}, \mathrm{N}, \mathrm{TE}$ & & 1976 & 1 \\
\hline 199 & Milk Creek at mouth, near Wolcott & USGS & 394240106423200 & 39.71164 & -106.70933 & $\mathrm{O}, \mathrm{FP}, \mathrm{MI}, \mathrm{AE}, \mathrm{S}, \mathrm{N}, \mathrm{TE}$ & & 1976 & 2 \\
\hline 200 & Milk Creek at I-70 near Wolcott & CDPHE & 12574 & 39.71115 & -106.70928 & FP, MI, AE, S, N, TE & & 2001 & 2 \\
\hline 201 & Eagle River below Milk Creek near Wolcott, $\mathrm{CO}$ & USGS & 394220106431500 & 39.70554 & -106.72142 & $\mathrm{AE}, \mathrm{O}, \mathrm{FP}, \mathrm{TE}, \mathrm{R}, \mathrm{MI}, \mathrm{S}, \mathrm{N}$ & & 1976-2007 & 106 \\
\hline 202 & Eagle River at Hwy 6 Bridge above Eagle, CO & USGS & 393937106485400 & 39.66026 & -106.81559 & FP, MI, N, TE & & 1995 & 1 \\
\hline 203 & Eagle River at Eagle & DOW-RW & 3297 & 39.65680 & -106.82516 & FP, MI, TE & & 1990-1997 & 16 \\
\hline 204 & Eagle River at Hwy 6 bridge & DOW-RW & 686 & 39.65679 & -106.82521 & $\mathrm{FP}, \mathrm{MI}, \mathrm{N}, \mathrm{TE}$ & & $1997-2006$ & 89 \\
\hline \multirow[t]{2}{*}{206} & Eby Creek at mouth near Eagle, CO, Site 1B & USGS & 393930106494001 & 39.65944 & -106.82975 & FP, TE, MI, N & & 1979 & 2 \\
\hline & & CDPHE & 12520 & 39.66000 & -106.82966 & FP, MI, AE, S, N & & 1999 & 1 \\
\hline \multirow[t]{2}{*}{208} & Eagle River above Brush Creek at Eagle, $\mathrm{CO}$ & USGS & 393852106503200 & 39.64856 & -106.84332 & AE, O, FP, MI, S, N, TE & & 2000 & 2 \\
\hline & & CDPHE & 12501 & 39.64859 & -106.84331 & FP, MI, AE, S, N, TE & & 1999-2001 & 10 \\
\hline 209 & West Brush Creek at Adam camp ground & USFS & $35-005$ & 39.46994 & -106.72910 & FP, MI, AE, N, TE & & 1974-1977 & 10 \\
\hline 210 & West Brush Creek above Sylvan Lake near Eagle & USGS & 392814106434600 & 39.47055 & -106.72935 & $\mathrm{O}, \mathrm{FP}, \mathrm{TE}, \mathrm{MI}, \mathrm{AE}, \mathrm{N}$ & & 1976 & 3 \\
\hline 211 & West Brush Creek below Sylvan Lake & USFS & 35-004 & 39.47917 & -106.73750 & FP, MI, AE, N, TE & & 1974-1977 & 8 \\
\hline \multirow[t]{3}{*}{212} & East Brush Creek at Yeoman Park, near Eagle, CO & USGS & 09067700 & 39.50415 & -106.67809 & FP, MI, TE & 1966-1972 & $1965-1972$ & 81 \\
\hline & & USFS & 09067700 & 39.50417 & -106.67804 & FP, MI, AE, N, TE & & $1973-1977$ & 12 \\
\hline & & USGS & 393045106404000 & & & $\mathrm{O}, \mathrm{FP}, \mathrm{MI}, \mathrm{AE}, \mathrm{S}, \mathrm{N}, \mathrm{TE}$ & & 1976 & 3 \\
\hline 213 & Nolan Creek at Middle Bridge & USFS & $35-006$ & 39.52599 & -106.69230 & FP, MI, AE & & 1973 & 1 \\
\hline 214 & East Brush Creek at Fisher Gulch & USFS & $35-002$ & 39.53750 & -106.74167 & FP, MI, AE, N, TE & & 1974-1977 & 11 \\
\hline 215 & East Brush Creek above confluence & USGS & 393221106450700 & 39.53920 & -106.75255 & $\mathrm{AE}, \mathrm{O}, \mathrm{FP}, \mathrm{MI}, \mathrm{S}, \mathrm{N}, \mathrm{TE}$ & & 2000 & 2 \\
\hline 216 & East Brush Creek at mouth near Eagle & USGS & 393227106451700 & 39.54082 & -106.75531 & $\mathrm{O}, \mathrm{FP}, \mathrm{TE}, \mathrm{MI}, \mathrm{AE}, \mathrm{S}, \mathrm{N}$ & & 1976-1977 & 4 \\
\hline
\end{tabular}


[no., number; USGS, U.S. Geological Survey; CDPHE, Colorado Department of Public Health and Environment; CO, Colorado; WWTP, wastewater treatment plant; CSU, Colorado Springs Utilities; GRC, Grand River Consulting, EMSSA, Eagle Mine Superfund Site Assessment; DOW-RW, Division of Wildlife, River Watch; CDNR, Colorado Division of Natural Resources; ASI, Advanced Sciences Incorporated; CDOT, Colorado Department of Transportation; ERWSD, Eagle River Water and Sanitation District; USFS, U.S. Forest Service; USEPA, U.S. Environmental Protection Agency; FP, field properties; MI, major ions; TE, trace elements; O, other constituents; AE, aquatic ecology; S, sediment; N, nutrients; R, radionuclide]

\begin{tabular}{|c|c|c|c|c|c|c|c|c|c|}
\hline $\begin{array}{l}\text { Site } \\
\text { no. }\end{array}$ & Site name & $\begin{array}{l}\text { Data } \\
\text { sources }\end{array}$ & $\begin{array}{c}\text { Site } \\
\text { identifiers }\end{array}$ & Latitude & Longitude & Type of data & $\begin{array}{c}\text { Period of } \\
\text { discharge record } \\
\text { (water year) }\end{array}$ & $\begin{array}{l}\text { Period of water- } \\
\text { quality record } \\
\text { (calendar year) }\end{array}$ & $\begin{array}{c}\text { No. of } \\
\text { samples }\end{array}$ \\
\hline \multirow[t]{2}{*}{217} & Brush Creek near Eagle, CO & USGS & 09068000 & 39.55721 & -106.76309 & FP, TE, R, MI & $1951-1972$ & 1960-2007 & 145 \\
\hline & & USFS & 09068000 & & & FP, MI, AE, N, TE & & 1973-1977 & 10 \\
\hline 218 & Salt Creek above Trail Gulch near Eagle, CO & USGS & 393558106452200 & 39.59943 & -106.75670 & FP, MI, TE & & 1971 & 1 \\
\hline 219 & Salt Creek below Brush Creek near Eagle, CO & USGS & 393655106453001 & 39.61420 & -106.76379 & FP, TE & & 1979 & 1 \\
\hline 220 & Abrams Creek & USGS & 393710106492001 & 39.61903 & -106.82055 & FP, MI, N, TE & & 1979 & 1 \\
\hline 221 & Brush Creek at Highway 6 & CDPHE & 12503 & 39.64622 & -106.84109 & FP, MI, AE, S, N, TE & & 2002 & 1 \\
\hline 222 & Brush Creek at Mouth at Eagle WWTP & CDPHE & 12530 & 39.64660 & -106.84117 & FP, MI, AE, S, N, TE & & 1999-2002 & 5 \\
\hline 223 & Brush Creek at Mouth near Eagle, CO & USGS & 393851106503400 & 39.64818 & -106.84362 & AE, O, FP, TE, MI, S, N & & 1976-2001 & 7 \\
\hline 224 & Eagle River at Gypsum, $\mathrm{CO}$ & USGS & 09069000 & 39.64999 & -106.95227 & AE, O, FP, TE, R, MI, S, N & & $1947-2007$ & 995 \\
\hline \multirow[t]{2}{*}{225} & Eagle River at Gypsum, CO & DOW-RW & 3298 & 39.64997 & -106.95230 & FP, MI, TE & & 1990-1997 & 51 \\
\hline & & DOW-RW & 3331 & 39.64997 & -106.95232 & FP, MI, TE & & 1992 & 1 \\
\hline 227 & Eagle River at Gypsum, CO & CDPHE & 000052 & 39.64989 & -106.95221 & FP, TE, R, MI, AE, S, N & & 1968-2005 & 368 \\
\hline 228 & Red Creek Tributary & USFS & $37-001$ & 39.47077 & -106.85146 & FP, MI, TE & & 1976 & 1 \\
\hline 229 & Gypsum Creek near Gypsum, CO & USGS & 09069500 & 39.54554 & -106.93477 & FP, TE, R, MI & 1951-1955, 1966-1972 & 1965-2007 & 88 \\
\hline \multirow[t]{2}{*}{230} & Gypsum Creek at Gypsum, CO & USGS & 393842106571900 & 39.64492 & -106.95077 & FP, MI, N, TE & & 1977 & 1 \\
\hline & & USGS & 393842106571901 & & & FP, MI, N, TE & & 1978 & 1 \\
\hline \multirow[t]{2}{*}{232} & Gypsum Creek at Gypsum, CO & USGS & 09069900 & 39.64887 & -106.95227 & AE, O, FP, TE, MI, S, N & & $1958-2002$ & 30 \\
\hline & & CDPHE & 12510 & 39.64887 & -106.95233 & FP, MI, AE, S, N, TE & & 1999-2002 & 6 \\
\hline 233 & Eagle River below Gypsum, CO & USGS & 09070000 & 39.64943 & -106.95366 & FP, MI, S, N, TE & 1947-2008 & 1959-2004 & 417 \\
\hline 234 & Eagle River below Gypsum, CO & DOW-RW & 42 & 39.65505 & -106.97821 & FP, MI, TE & & $1990-1993$ & 15 \\
\hline \multirow[t]{2}{*}{235} & Eagle River at Mouth, near Dotsero, CO & USGS & 09070400 & 39.64615 & -107.05674 & FP, R, MI & & $1969-2006$ & 8 \\
\hline & & USEPA & 09070400 & & & $\mathrm{FP}, \mathrm{O}, \mathrm{TE}$ & & 1969-1972 & 8 \\
\hline
\end{tabular}




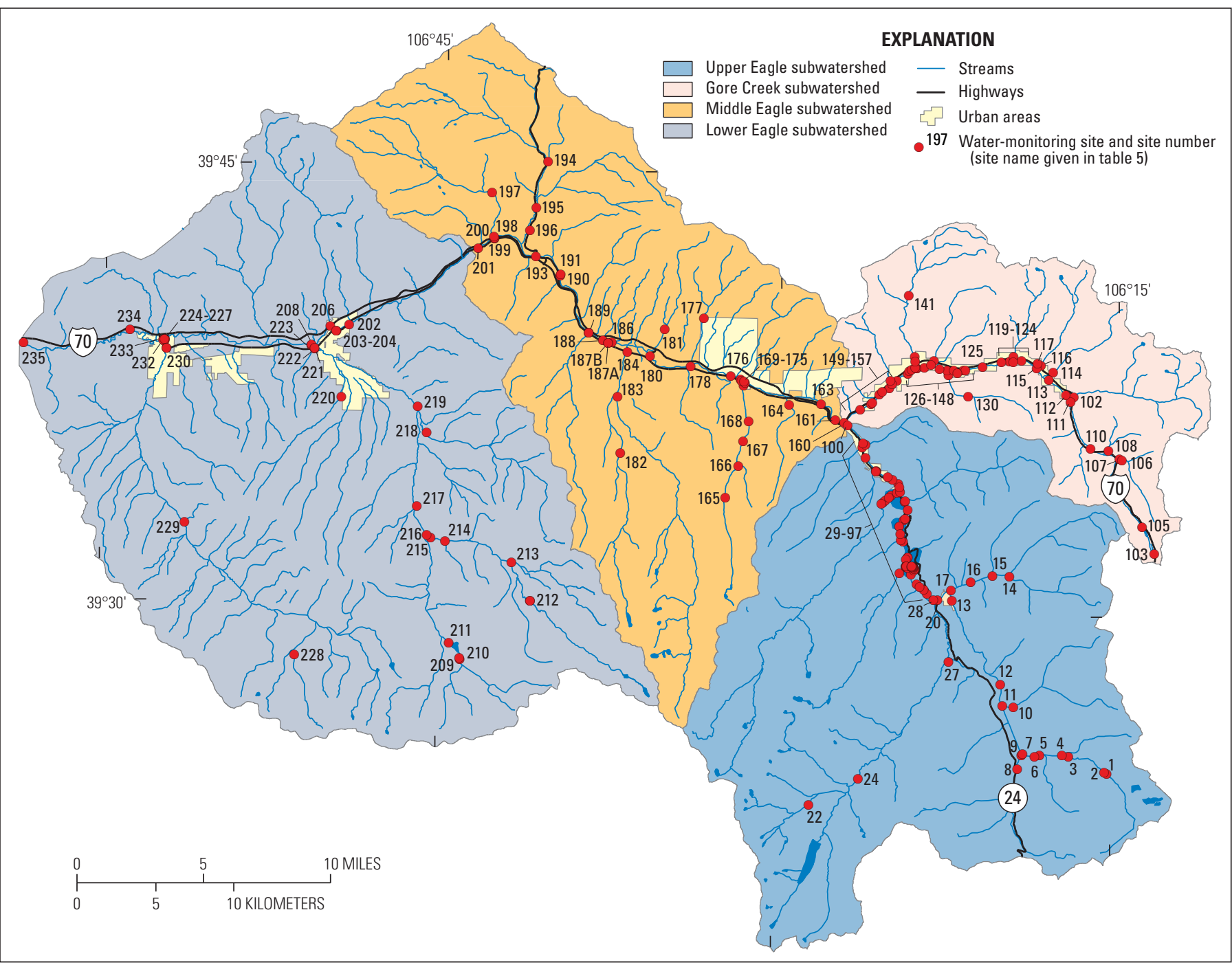

Base from U.S. Geological Survey digital data, 2007,

Universal Transverse Mercator projection

Zone 13

Figure 7. Locations of surface-water-monitoring sites within the Eagle River watershed, Colorado, 1947-2007. 


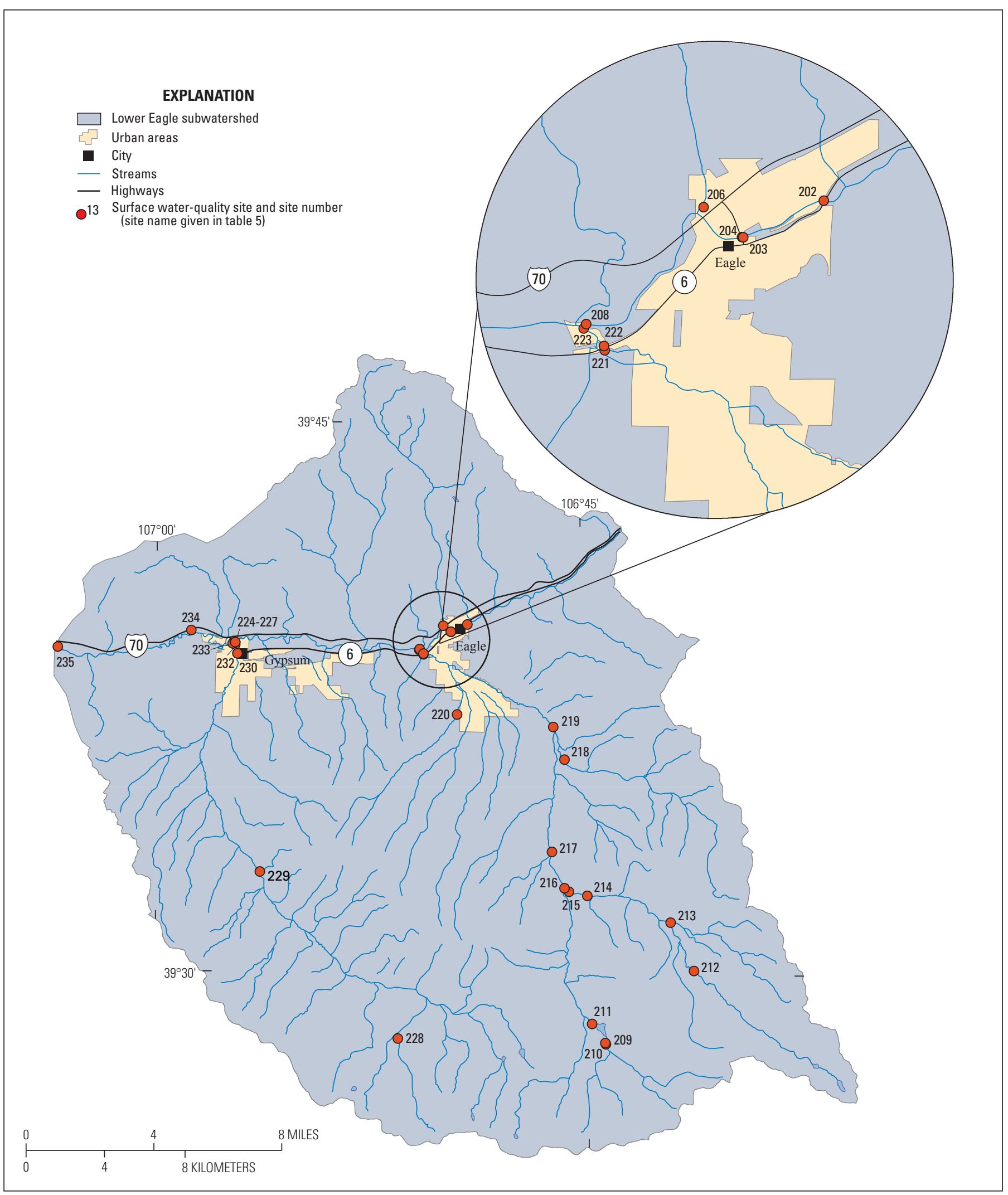

Base from U.S. Geological Survey digital data, 2007,

Universal Transverse Mercator projection

Zone 13

Figure 8. Locations of surface-water-monitoring sites in the lower Eagle River subwatsershed, Colorado, 1947-2007. 


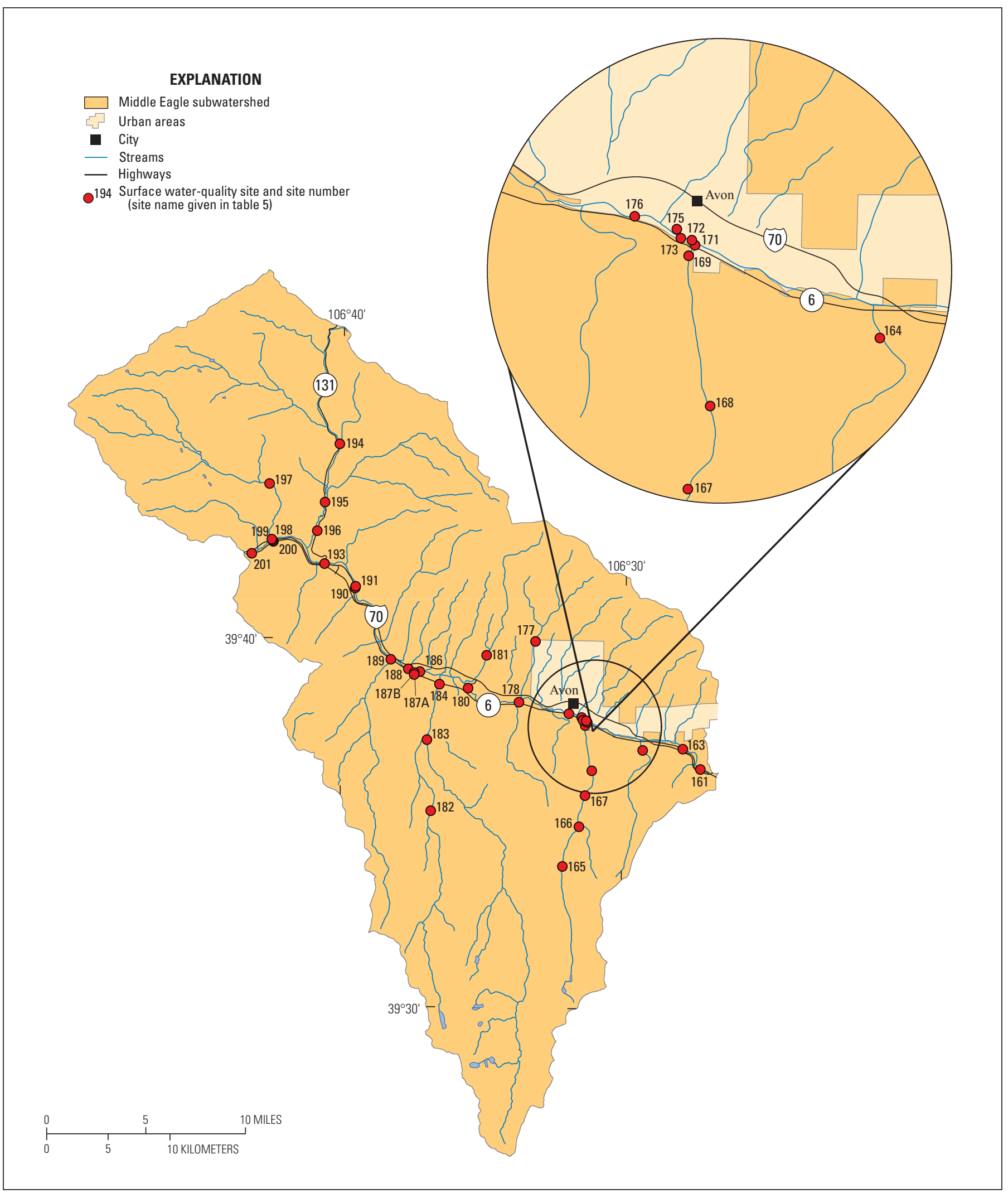

Base from U.S. Geological Survey digital data, 2007,

Universal Transverse Mercator projection

Zone 13

Figure 9. Location of surface-water-monitoring sites in the middle Eagle River subwatershed, Colorado, 1947-2007. 


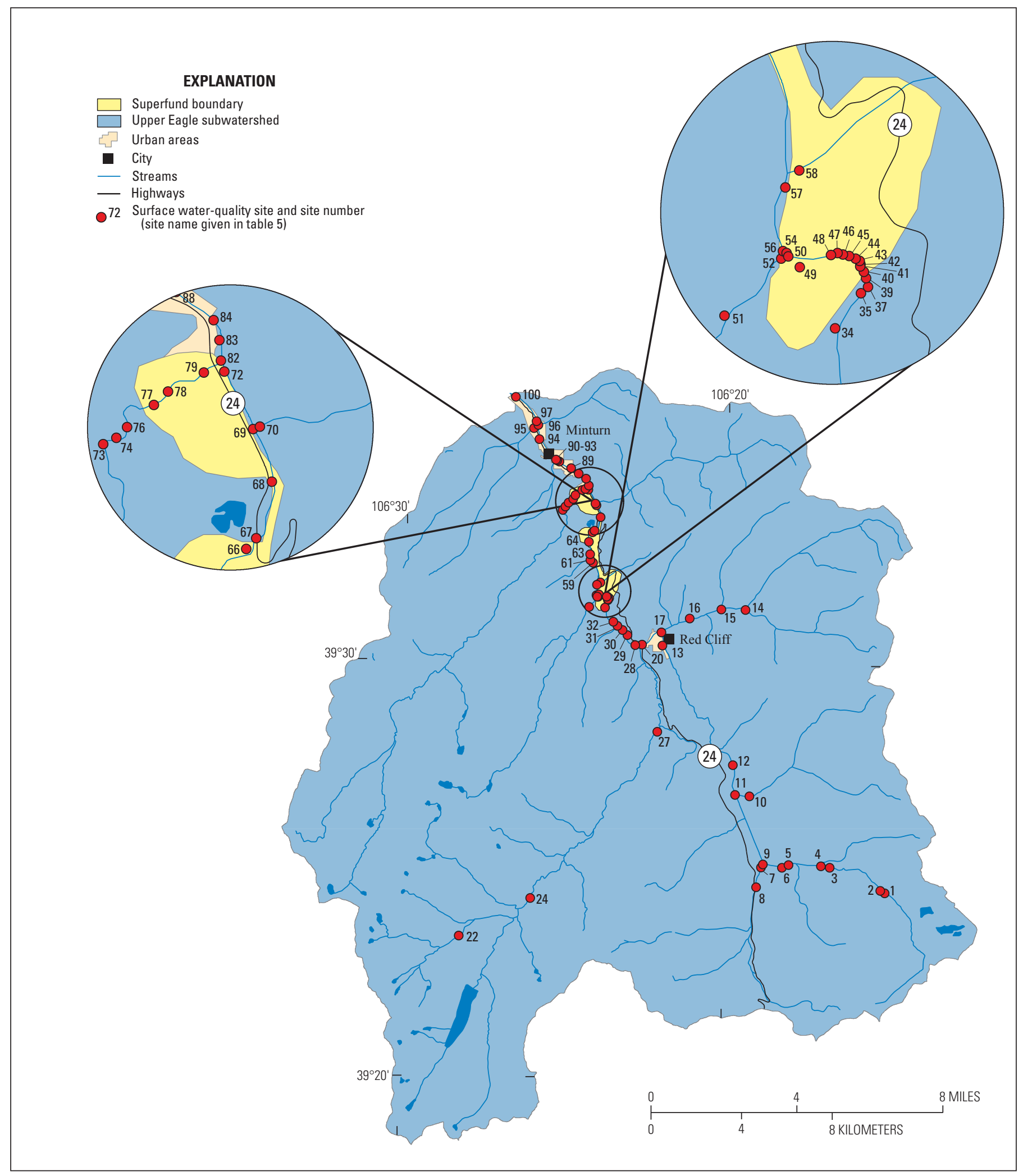

Base from U.S. Geological Survey digital data, 2007,

Universal Transverse Mercator projection

Zone 13

Figure 10. Location of surface-water-monitoring sites in the Upper Eagle River subwatershed, Colorado, 1947-2007. 


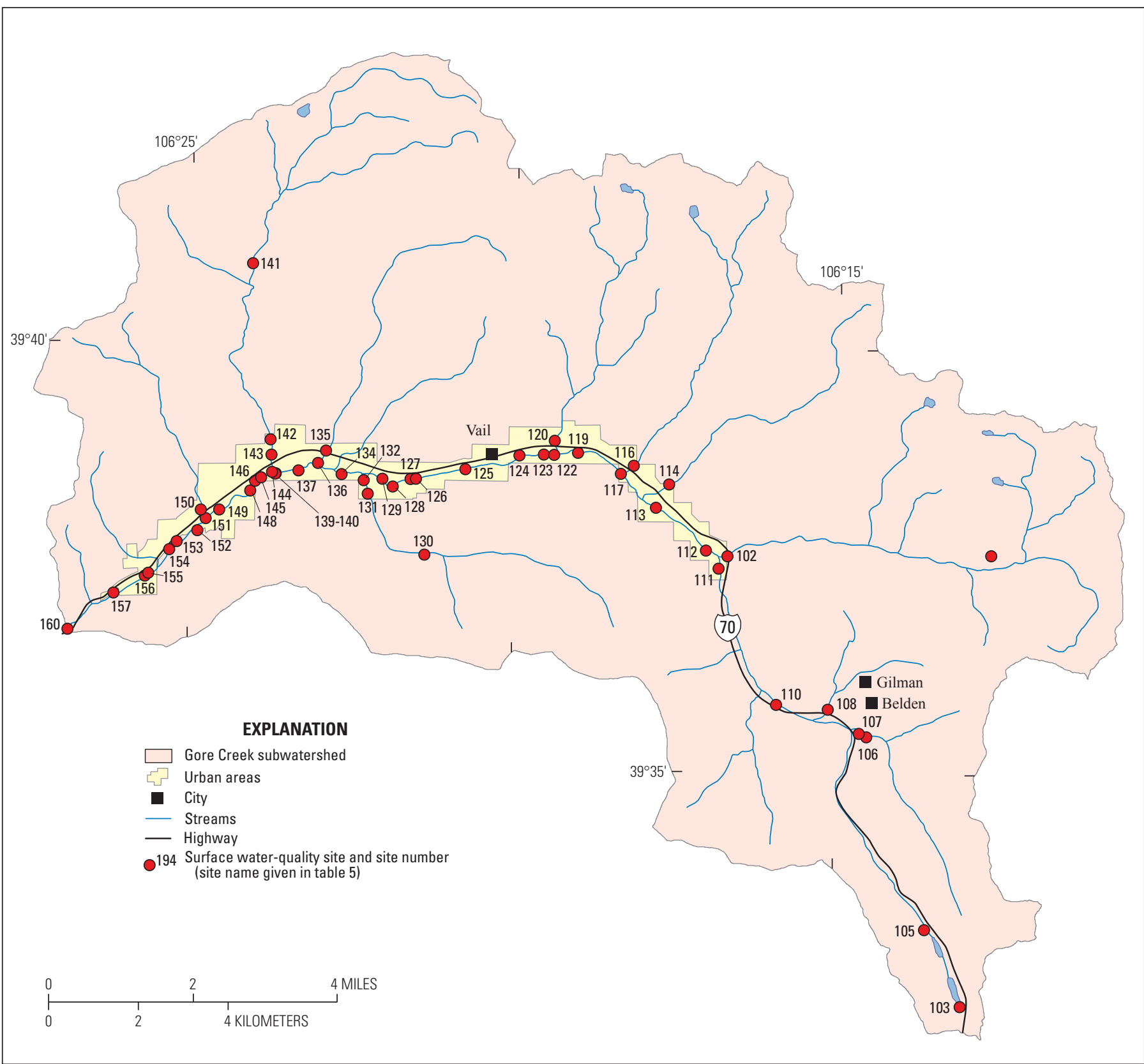

Base from U.S. Geological Survey digital data, 2007,

Universal Transverse Mercator projection

Zone 13

Figure 11. Location of surface-water-monitoring sites in the Gore Creek subwatershed, Colorado, 1947-2007. 


\section{Surface-Water Quality}

\section{Data Sources and Compilation}

Available surface-water quality data were obtained from local, State, and Federal agencies and entered into a relational database to assist in the analysis of water-quality conditions in the ERW 1947-2007. Digital data were obtained from the USEPA web-based STORage and RETrieval (STORET, http://www.epa.gov/storet/dbtop.html) system, the USGS web-based National Water Information System (NWIS, http://waterdata.usgs.gov/nwis), and from local agencies and stakeholders. The data used in the analysis described in this report are available in a web-based repository at http://rmgsc. cr.usgs.gov/cwqdr/Eagle/index.shtml (5/21/2011). A summary of the data sources and data abundance is provided in table 1.

Incorporation of multiple-data sources into a common repository required combining data into a single, uniform data structure. Data compilation, including changes to data format and consistent scaling for each parameter code as well as aggregation of similar constituents into common parameter codes, was done to homogenize the datasets and simplify the data structure. The determination of the chemical properties of waters can result in censored data because of analytical limits of the laboratory analysis. Censored data provide a range of possible values expressed as (1) less than the reporting limit, or (2) values greater than the maximum value reported. For left-censored data in this report (data reported as less than the reporting limit), the most common reporting limit (most frequently used) for each parameter code was kept; censored values greater than the common reporting limit were deleted; censored values below the common reporting limit were converted to the common reporting limit. For right-censored data in this report (data reported as greater-than the maximum value reported), the most common maximum value reported (most frequently used) for each parameter code was kept; the values greater than the maximum value reported were set equal to the censored value. In addition, aggregation of parameters was done following guidance outlined in Mueller and others (1995) to simplify the analysis procedures. This aggregation was done for nutrients such as nitrate, nitrite, ammonia, un-ionized ammonia, total phosphorous, and orthophosphate.

Spatial comparisons of water-quality site locations were done to determine if collection of water-quality samples occurred at the same location under different site identifications. Aggregation of multiple sites into a single identifier provides greater efficiency and increased statistical merit during analysis. The validity of the aggregation of sites was determined by evaluation of the hydrologic complexity of a location and its proximity to additional site locations. Care was taken when aggregating two sites with the same source agency; it was assumed that each agency already had taken care not to duplicate sites unnecessarily. In some instances, however, aggregating more than one site from the same source agency was deemed warranted. This aggregation most often was done when two sites were in close proximity, and one of the sites had few samples (one or two samples as compared with dozens). A summary of the available data for these sites along with the aggregation of these sites is presented in table 5 and figures 7-11. When sites are referenced throughout this report, the subwatershed is included in addition to the site number.

\section{Data Review and Analysis}

Review of the water-quality datasets was completed prior to analysis through several quality-assurance methods. Initial data review began with identification of gross errors in the data through use of USEPA guidelines for typical ranges of values for constituents and properties of natural waters (National Park Service, 1998). Values outside these identified ranges provided a basis to characterize samples as suspect. In natural waters, the electrostatic charges of positive ions (cations) and negative ions (anions) are balanced. The ioniccharge balance of a water-quality sample was evaluated for samples with sufficient data (at a minimum samples must have values reported for calcium, magnesium, sodium, potassium, alkalinity (or acid-neutralizing capacity), sulfate, and chloride) using methods outlined in Hem (1989). Samples with excessive differences in charge-balances (usually greater than 5-percent difference) were considered suspect and reviewed to determine if they should be removed from the dataset. An additional comparison between filtered (dissolved) and unfiltered (total) concentrations for a constituent also were applied to samples that had both analytical results available. If the filtered concentrations were greater than the unfiltered concentrations, the samples were removed from the dataset.

Samples also were reviewed in the context of geologic setting, land use, season, consistency, and comparison to values from adjacent sites for validation. Questionable or inconsistent samples were removed from the dataset. Quality assurance results are presented in table 6. Samples lacking sufficient analytical results for ion-balance and filteredunfiltered comparisons were assumed to be accurate if the values were within the range of typical values. A large portion of the data has limited quality assurance review and may contain errors. Further exploration of the dataset was beyond the scope of this analysis.

This report provides reconnaissance-level statistical summaries and comparisons of water-quality conditions and characteristics using available data within the ERW. An inclusive approach was taken to expand the number of samples and sites available for analysis in order to provide greater spatial and temporal coverage of water-quality conditions in the watershed. A large portion of the data has limited metadata and (or) quality-assurance data. Therefore, it is possible that some data may contain errors that were not detected during the waterquality data review. Assumptions regarding water-quality collection methods and laboratory-analytical techniques used on data from different data sources were made based on available information. No distinctions between water-quality data collection methods and laboratory-analytical techniques were made when metadata were unavailable to support these distinctions. 
Disparities between data from different sources resulting from differences in water-quality collection methods and laboratoryanalytical techniques may affect the precision and accuracy of the statistical results. Although the effect of methodological differences could not be quantified in this analysis, robust statistical methods were employed to limit the effect of outliers on statistical results of the analysis.

Summary statistics in this report were calculated using methods described in Helsel (2005) and in Helsel and Hirsch (1992) based on the number of samples available and the percentage of the data that was left-censored (nondetects). Summary statistics for minimum, 25th, 50th (median), and 75 th percentiles, and maximum are calculated using traditional statistical methods when the datasets included only uncensored data. For datasets with less than 50 percent nondetects, the Kaplan-Meier method (Helsel and Hirsch, 1992) was used; where 50-80 percent of the data were nondetects and there were more than 50 samples, the adjusted maximum likelihood estimator was used (Helsel, 2005).

Graphical representations of water-quality results are presented in this report using boxplots, bar graphs, line graphs, and maps. Boxplots are used to show the ranges of concentrations observed within particular groupings of data. These plots show a range of data including the 25th, 50th (median), and 75th percentiles of data for groupings with more than four samples. In map form, depictions of the median constituent concentrations from sampling sites within the ERW are displayed for sites with a minimum of five samples and a time span of 5 years between the first and last samples. These medians are normalized to the percentiles of data from the whole watershed as follows: (1) for sample-site medians less than the 25 th percentile, (2) for sample-site medians between the 25 th and less than the 50th percentile, (3) for sample-site medians between the 50 th and less than the 75 th percentile, and (4) for sample-site medians equal to or greater than the 75th percentile.

Determination of temporal trends for select constituents was done using nonparametric statistical methods. Nonparametric statistical methods were selected because the statistical results are not strongly affected by outliers, and there are minimal assumptions regarding the distribution shape of the datasets (Helsel and Hirsch, 1992). Step-trend and monotonic-trend analyses were performed. Step trends are appropriate when identifiable, abrupt changes occur in either the hydrologic system or in the source area for water-quality constituents, resulting in two discrete groups of data (Helsel and Hirsch, 1992). For step-trend assessments, the Wilcoxon rank-sum test was used to compare the median values between two time periods defined as pre- and post-change periods (Helsel and Hirsch, 1992). The Peto-Prentice test was used if the concentration data included nondetects.

Monotonic-trend assessments (single-direction, upward, or downward) were done on selected water-quality constituents to identify changes to stream conditions at sites with

Table 6. Eagle River watershed quality-assurance results by sample source agency.

[No., number; USEPA, U.S. Environmental Protection Agency; --, no data; Count, the number of samples compared to the quality-assurance test; Rejected, the number of samples that failed the quality-assurance test]

\begin{tabular}{|c|c|c|c|c|}
\hline \multirow[t]{2}{*}{ Source agency } & \multirow{2}{*}{$\begin{array}{c}\text { No. of } \\
\text { samples }\end{array}$} & $\begin{array}{c}\text { Charge-balance } \\
\text { equation }\end{array}$ & \multirow{2}{*}{$\begin{array}{c}\begin{array}{c}\text { Filtered } \\
\text { and unfiltered }\end{array} \\
\text { Count/rejected }\end{array}$} & \multirow{2}{*}{$\begin{array}{c}\begin{array}{c}\text { USEPA Typical } \\
\text { Range Guideline }\end{array} \\
\text { Count/rejected }\end{array}$} \\
\hline & & Count/rejected & & \\
\hline Advanced Sciences Inc. & 299 & $--/--$ & $282 / 18$ & $299 / 0$ \\
\hline Colorado Department of Natural Resources & 171 & $--/--$ & $--/--$ & $171 / 0$ \\
\hline Colorado Department of Public Health and Environment & 2,340 & $--/--$ & $336 / 0$ & $2,340 / 0$ \\
\hline Colorado Springs Utilities & 262 & $--/--$ & $58 / 1$ & $262 / 0$ \\
\hline Division of Wildlife (River Watch) & 539 & $--/--$ & $374 / 133$ & $539 / 0$ \\
\hline Eagle Mine Superfund Site Assessment & 2,116 & $--/--$ & $819 / 209$ & $2,116 / 0$ \\
\hline Eagle River Water and Sanitation District & 997 & $--/--$ & $6 / 1$ & $997 / 0$ \\
\hline Grand River Consulting & 26 & $--/--$ & $25 / 0$ & $26 / 0$ \\
\hline
\end{tabular}


sufficient data. To account for sampling bias and to ensure independence of the sample data, a subset of the data was used in each trend test. The subset limited the data to one sample each month (step-trend analysis) or one sample each season (monotonic-trend analysis) within the same calendar year in order to meet the assumptions and requirements, or both, of each test. Sites with sufficient data had (1) adequate seasonal characterization, (2) a minimum of 7-years of data with at least one sample collected after January 1, 2003, (3) less than 50 percent censoring of the data, and (4) adequate distribution of samples in the first and last one-third of the record (Lanfear and Alexander, 1990). Monotonic trends in concentration data were tested using the seasonal Kendall test (Helsel and Hirsch, 1992). The seasonal Kendall test accounts for seasonal effects in the concentration data through the separation and comparisons of data by each season. Where adequate streamflow data are available, and the amount of censored water-quality data is less than 8 percent, flow-adjusted concentration data were used in the trend analysis to account for the variation in concentrations that is related to streamflow (Helsel and Hirsch, 1992). Trends in unadjusted concentrations may be due to trends in concentrations or trends in streamflow quantity or timing. Flow-adjusted analysis better quantifies changes in constituent concentrations over time that are not the result of changes in streamflow; therefore, where both trend tests have been evaluated, preference is given to flow-adjusted trends. Trend tests for cadmium, iron, manganese, and zinc excluded data from February and March for each year because of observed variability during this time period attributed to known hydrological processes (Colorado Department of Public Health and Environment and U.S. Environmental Protection Agency, 2000) that may not be well characterized based on assumptions and procedures used in the seasonal Kendall test, the Wilcoxon rank sum test, and the Peto-Prentice test.
In this report, temporal trends were evaluated based on statistical significance and are considered highly significant when the trend test reports a greater than 99-percent confidence level (p-value less than 0.01), significant when the trend test reports a greater than 95-percent confidence level (p-value less than 0.05), and weakly significant when the trend test reports a greater than 85-percent confidence level (p-value less than 0.15). For all trend-test results, the calculated level of significance (p-value) is given; for trend-test results with p-values less than 0.15 , the trend direction (upward or downward) is reported; for highly significant and significant trends, the slope or magnitude of the trend also is reported.

The trend magnitude from the seasonal Kendall test is the median slope of all pair-wise comparison of points used in the seasonal Kendall test. This slope is not the amount of change expected to be observed each year and does not require that the change in concentrations recognized in the test occur in any distribution within the trend period, so it is possible for a linear or nonlinear trend to be occurring. As a result, the slope better describes the trend observed over the entire trend period instead of an average annual slope. The trend slope should not be used to forecast future stream conditions.

Temporal trends have both a statistical significance and environmental significance. The trend magnitude provides information on the significance of the trend from an environmental perspective, but often is a subjective decision based on the context of the current stream conditions. It is important when reviewing trend summaries to consider the specific constituent of concern and any corresponding standard.

The Colorado Water Quality Control Commission (CWQCC), supported by the CDPHE, sets stream classifications and water-quality standards for rivers and other water bodies across the state; they also are required under Section 303(d) of the Federal Clean Water Act to submit a list of

Table 7. Federal Clean Water Act Section 303(d) listings for the Eagle River watershed.

[Cd, cadmium; Mn, manganese; $\mathrm{Zn}$, zinc; $\mathrm{Cu}$, copper; --, no data]

\begin{tabular}{|c|c|c|c|c|c|c|c|}
\hline \multirow{2}{*}{ Reach } & \multicolumn{6}{|c|}{ Federal Clean Water Act Section 303(d) listing cycle ${ }^{1}$} & \multirow[b]{2}{*}{2008} \\
\hline & 1996 & 1998 & $2000^{2}$ & 2002 & 2004 & 2006 & \\
\hline Black Gore Creek & -- & -- & -- & $\begin{array}{l}\text { Sedimentation/ } \\
\text { siltation }\end{array}$ & $\begin{array}{l}\text { Sedimentation/ } \\
\text { siltation }\end{array}$ & $\begin{array}{l}\text { Sedimentation/ } \\
\text { siltation }\end{array}$ & $\begin{array}{l}\text { Sedimentation } \\
\text { siltation }\end{array}$ \\
\hline Eagle River-Belden to Gore confluence & -- & $\mathrm{Cd}, \mathrm{Mn}, \mathrm{Zn}$ & - & $\begin{array}{c}\mathrm{Mn}, \mathrm{Zn} \text { (entire } \\
\text { reach), and } \mathrm{Cu} \\
\text { (above Rex Flats) }\end{array}$ & $\mathrm{Cu}, \mathrm{Zn}$ & $\mathrm{Cu}, \mathrm{Zn}$ & $\mathrm{Cu}^{3}, \mathrm{Zn}$ \\
\hline $\begin{array}{l}\text { Eagle River-Gore confluence to } \\
\text { Colorado River }\end{array}$ & $\begin{array}{l}\text { Metals (other } \\
\text { than mercury) }\end{array}$ & $\mathrm{Mn}$ & -- & -- & -- & -- & -- \\
\hline
\end{tabular}


identified waters within Colorado which have "water-qualitylimited segments still requiring Total Maximum Daily Loads" (Colorado Department of Public Health and Environment, 2009). The water-quality standards are established to protect specific classified uses. In addition, numeric standards exist for physical, biological, inorganic, and metal constituents. Comparisons of water-quality conditions to available water-quality standards were done to provide environmentally relevant context to observations of stream conditions. The objective of this report is not to characterize or rate the "condition" of any stream, or to assess the impairment or health of the watershed. Available information on Section 303(d)-listed stream segments within the ERW is presented in table 7 to provide additional context to aid in the determination of the environmental significance of water quality in the ERW.

Surface-water-quality data were compared to CDPHE in-stream water-quality State standards, which are based on the stream-reach classification. The stream segments within the ERW (segments 1, 2, 3, 4, 5a, 5b, 5c, 6, 7a, 7b, 8, 9, 10, 11, and 12) are classified for cold-water aquatic life, recreational, domestic-water supply, and agricultural use. Stream segments generally are delineated at points on a water course that separate reaches with significant differences in classification and change, or both, in water-quality standard (Colorado Department of Public Health and Environment, 2008). Figure 12 shows the location of defined stream segments in the ERW.

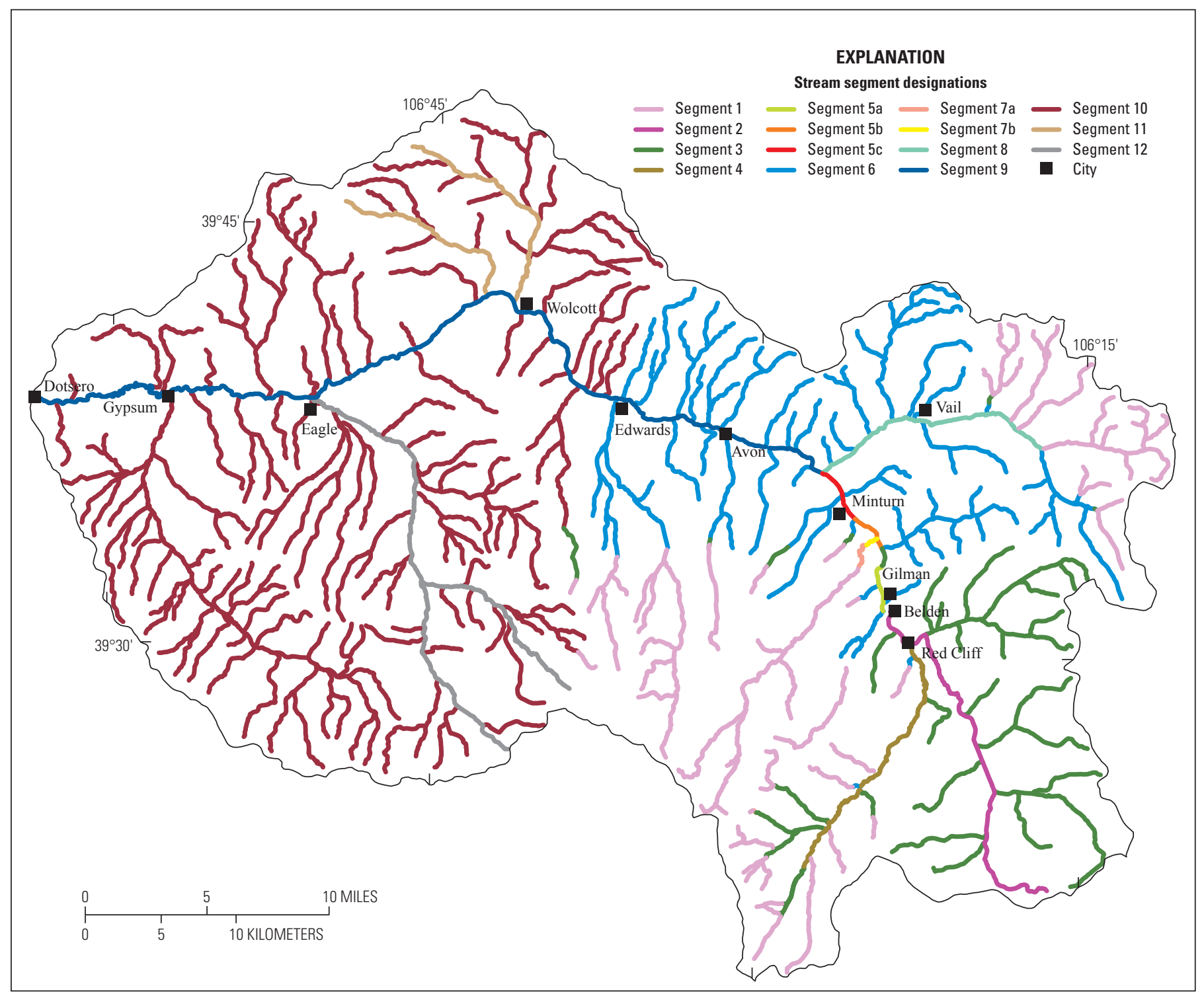

Base from U.S. Geological Survey digital data, 2007,

Universal Transverse Mercator projection

Zone 13

Figure 12. Colorado Department of Public Health and Environment stream-segment designations for the Eagle River watershed, Colorado (Colorado Department of Public Health and Environment and U.S. Environmental Protection Agency, 2008). 
Many State water-quality standards use Table Value Standards (TVS), some of which are based on site-specific hardness calculations. Calculations of hardness values at each site resulted from four different calculation methods from a regression analysis of site-specific data (Colorado Department of Public Health and Environment, 2008). The following calculations were done in decreasing order of preference: (1) for sites with 6 or more hardness samples during the low-flow period, hardness values were calculated as the lower 95th confidence interval about the mean hardness value for only the samples from the low-flow period; (2) for sites with 1-5 hardness samples during the low-flow period, hardness values were calculated as the mean hardness value for only those samples collected during the low-flow period; (3) for sites without low-flow hardness samples and 6 or more hardness samples, hardness values were calculated as the lower 95th confidence interval about the mean hardness value for all samples; (4) for sites without low-flow hardness samples and less than 6 hardness samples, hardness values were calculated as the mean hardness value for all samples.

CDPHE has established State standards for acute and chronic exposure levels for many constituents of in-stream water quality (http://www.cdphe.state.co.us/regulations/ wqccregs/index.html). The acute State standard refers to the level that is not to be exceeded by the concentration for either a single sample or is calculated as an average of all samples collected during a one-day period. The chronic State standard is defined as the level not to be exceeded by the concentration for either a single representative sample or calculated as an average of all samples collected during a thirty-day period. State standards for water temperature (chronic) in the ERW are specific to CDPHE Regulation 33 (effective September 1, 2007) designating stream reaches above and below elevations of $7,000 \mathrm{ft}$.

\section{Assessment of Surface-Water Quantity}

Streamflow conditions of the Eagle River follow a consistent seasonal pattern. Low-flow conditions (base flow) typically occur in October-March, with the start of the snowmelt-runoff generally beginning in late March and early April (fig. 13). As the snowpack begins to melt, streamflows begin to rise and increase to peak streamflow conditions in late May and early June. As available snowpack levels are depleted in late spring and early summer, streamflow levels return to the base-flow conditions of the fall (fig. 13). Occasional increases in flow during the summer and fall months can occur because of precipitation events within the ERW.

Many sites within the ERW show patterns of wet and dry years. The mid 1980s and 1990s are two wet periods within the last several decades, separated by average to dry periods in 1990 and 2000 (fig. 14). The wettest and driest years on record at most sites are 1984 and 2002, respectively (U.S. Geological Survey, 2007). Seasonal and multiyear patterns in streamflow can affect water quality through chemical processes and through dilution. Understanding the seasonal and multi-year patterns of streamflow within the watershed is important in interpreting patterns in water quality.

Variations in snowmelt timing can be important to water managers and can affect the streamflow characteristics throughout the year. Understanding runoff intensity and timing is important in order to properly manage the storage and release of snowmelt runoff in reservoirs. If snowmelt runoff is different than expected and if storage opportunities are missed, decreased water availability may result later in the year when municipal and agriculture demands increase.

The Eagle River contributes an average of 409,900 acre$\mathrm{ft}$ per year to the Colorado River system (based on USGS streamflow-gaging station Eagle River below Gypsum, CO, 09070000, water years 1947-2007; U.S. Geological Survey, 2007). Much of the water that flows out of the ERW originates from snow accumulations during the winter months. The ERW is a complex system of tributaries, lakes, and reservoirs that supply year-round water for agriculture, industrial, and municipal uses. The largest storage structure in the ERW is Homestake Reservoir (45,000 acre-ft), located in the southeast part of the ERW (fig. 15). There are several other smaller reservoirs in the watershed used for recreation, irrigation, snowmaking, flow augmentation, and domestic-water supply.

Homestake Reservoir is part of the transmountaindiversion system that moves water through the Continental Divide to supply water to cities east of the Continental Divide (U.S. Geological Survey, 2008). Diversions from the ERW into the reservoir began in 1966. Water is then conveyed through the Homestake Tunnel to the Arkansas River Basin, thereby depleting the ERW by an average of 30,580 acre-ft per year (fig. 15). Records indicate that the largest diversions from Homestake Reservoir occur May-July, with smaller diversions sporadically throughout the rest of the year (Colorado Division of Water Resources, 2009). The Homestake Tunnel is the largest diversion from the ERW and spans 5.2 miles. The Ewing Ditch (constructed 1880), Wurtz Ditch (constructed 1929), Columbine Ditch (constructed 1931), also divert water from the upper Eagle River watershed across the Continental Divide into the Arkansas River Basin (Eagle River Watershed Plan, 1996). Average annual diversion totals from other diversion structures include the Wurtz Ditch with 2,210 acre-ft; the Columbine Ditch with 1,520 acre-ft; and the Ewing Ditch with 820 acre-ft (fig. 15). Diversions in these and other ditches typically occur May-October and represent less than 8 percent of the annual total streamflow that originates in the ERW (fig. 15).

Homestake Reservoir also supplies water to the ERW. Releases from the reservoir are important to urban centers within the ERW. Large releases from the reservoir occur at two times during the year, March-April, and September-October.

Dividing the ERW into smaller subwatersheds is a useful way to compare streamflow distributions and to understand water balances within the watershed. For this report, the ERW was divided into four subwatersheds: the Upper Eagle, Gore Creek, Middle Eagle, and Lower Eagle subwatersheds 

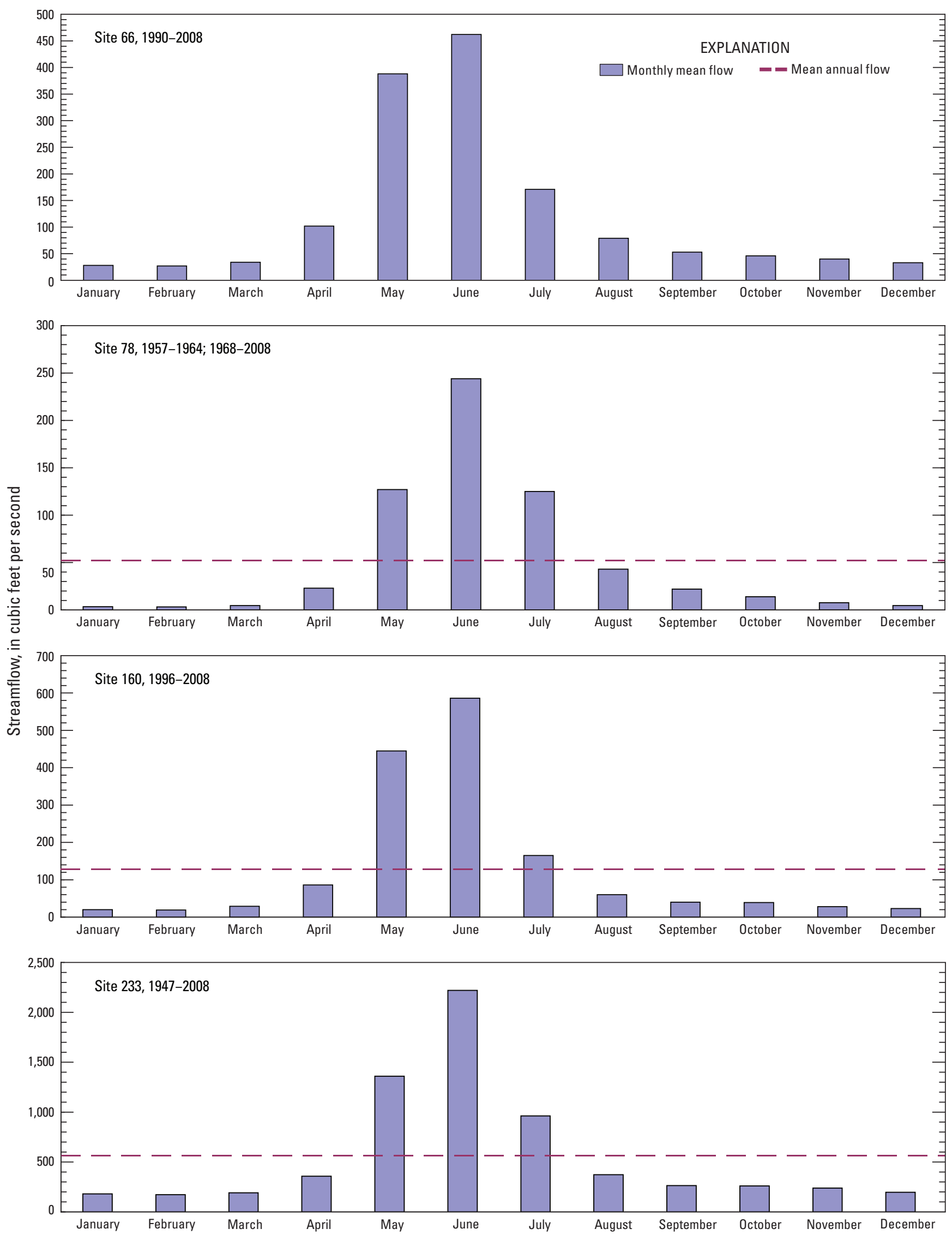

Figure 13. Hydrographs of selected sites in the Eagle River watershed, Colorado. 


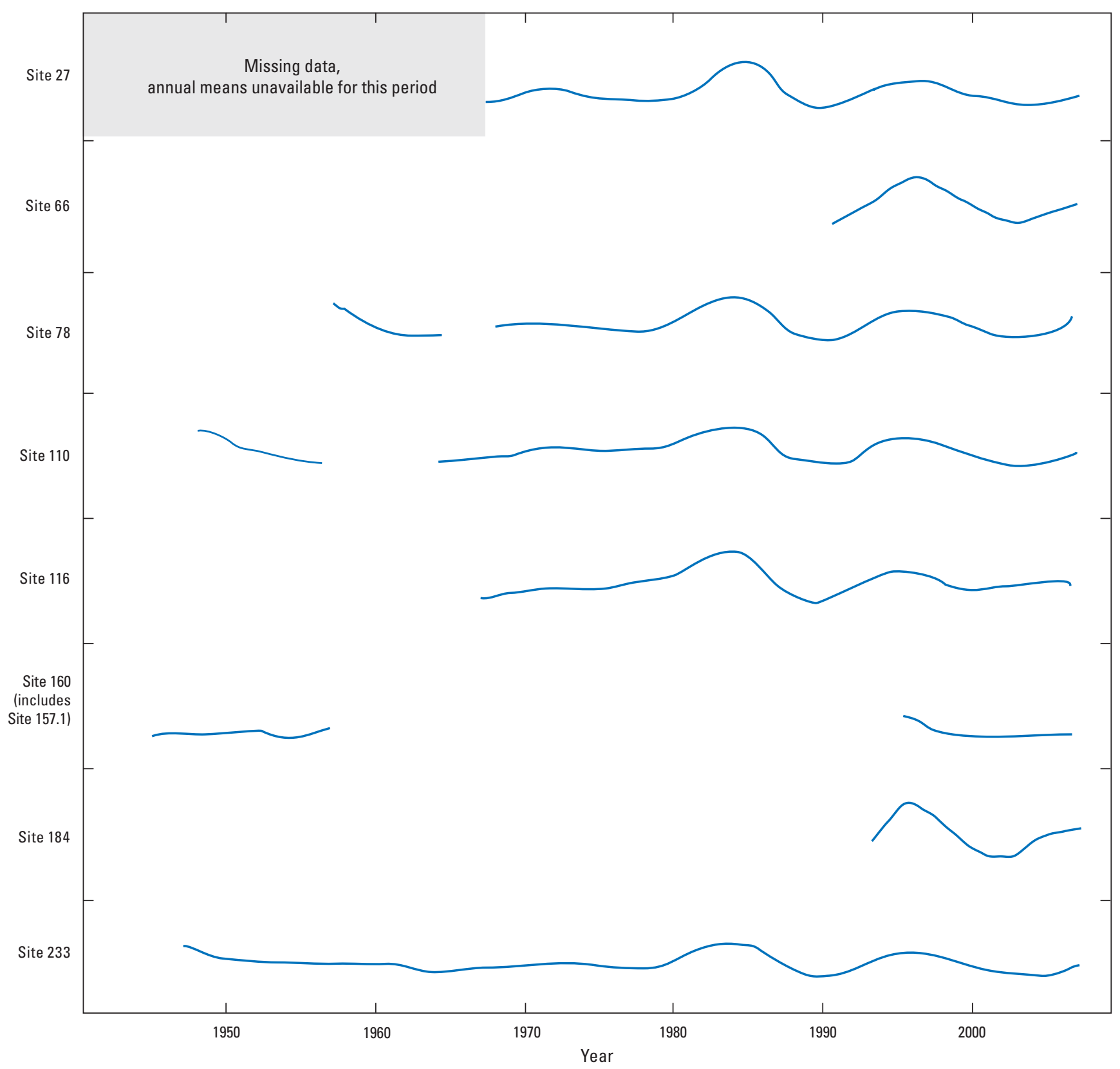

Figure 14. Lowess Smooth of annual-mean streamflow at selected sites in the Eagle River watershed, Colorado, $1947-2007$. 


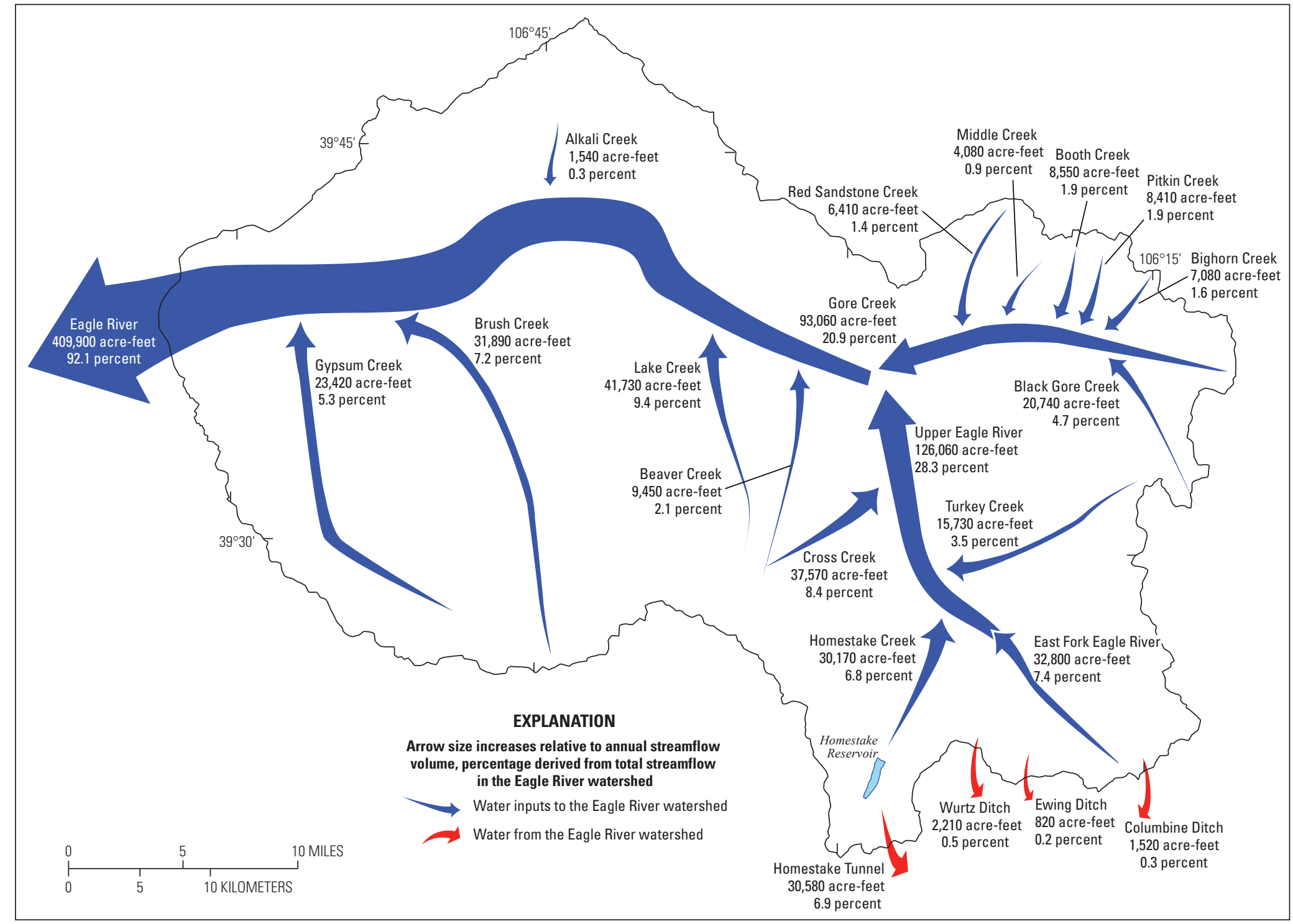

Base from U.S. Geological Survey digital data, 2007 ,

Universal Transverse Mercator projection

Zone 13

Figure 15. Arrow schematic of major streams and diversion mean annual streamflow volume in the Eagle River watershed. 
(fig.7-11). Comparisons of water quantities within the ERW show that 92 percent of the streamflow that originates within the watershed is accounted for at site 233 (Lower Eagle subwatershed) (09070000, Eagle River below Gypsum, CO). The remaining 8 percent is diverted near the headwaters to supplement water supplies east of the Continental Divide in Colorado (fig. 1 and fig. 15).

The Upper Eagle subwatershed contributes 126,060 acre$\mathrm{ft}$ annually (U. S. Geological Survey, 2007); this mean annual streamflow volume represents approximately 28.3 percent of the total annual streamflow volume that originates within the ERW for the period of record (fig. 15). It is the only subwatershed with transmountain diversions. In 2009 seven active streamflow-gaging stations in the Upper Eagle subwatershed. Major tributaries within the Upper Eagle subwatershed include Homestake Creek, Cross Creek, and Turkey Creek; contributing 30,170 acre-ft; 37,570 acre-ft; and 15,730 acre-ft, respectively (fig. 15).

The Gore Creek subwatershed contributes 93,060 acre-ft. The Gore Creek subwatershed contributes 20.9 percent of the annual streamflow volume that originates within the ERW. The Gore Creek subwatershed currently (2009) has three active streamflow-gaging stations with numerous discontinued stations, many of which were discontinued in 2008 (table 3, fig. 6). Major tributaries of Gore Creek include Black Gore Creek, Bighorn Creek, Pitkin Creek, Booth Creek, Middle Creek, and Red Sandstone Creek. The largest mean annual streamflow volume of these tributaries is Black Gore Creek with 20,740 acre-ft (fig. 15).

The combined Middle and Lower Eagle subwatersheds contribute 43 percent of the mean annual streamflow volume originating within the ERW and are currently (2009) monitored by 5 active streamflow-gaging stations. Many USGS streamflow-gaging stations in the Lower and Middle Eagle subwatersheds were discontinued in 1972 (table 3, fig. 6). The largest mean annual streamflow volumes within the Middle Eagle subwatershed are from Lake Creek with 41,730 acre-ft; and in the Lower Eagle subwatershed are from Brush Creek with 31,890 acre-ft (fig. 15).

The effects of diversions in the Upper Eagle subwatershed, primarily from Homestake Reservoir, are evident when comparing pre-diversion and post-diversion flow record at Homestake Creek near Red Cliff, Colorado (Upper Eagle subwatershed, site 27; fig. 6) in figure 16. Streamflow May-July decreased during the post-diversion period. Base flow through the summer, fall, and winter months remained similar to the pre-diversion period (fig. 16).

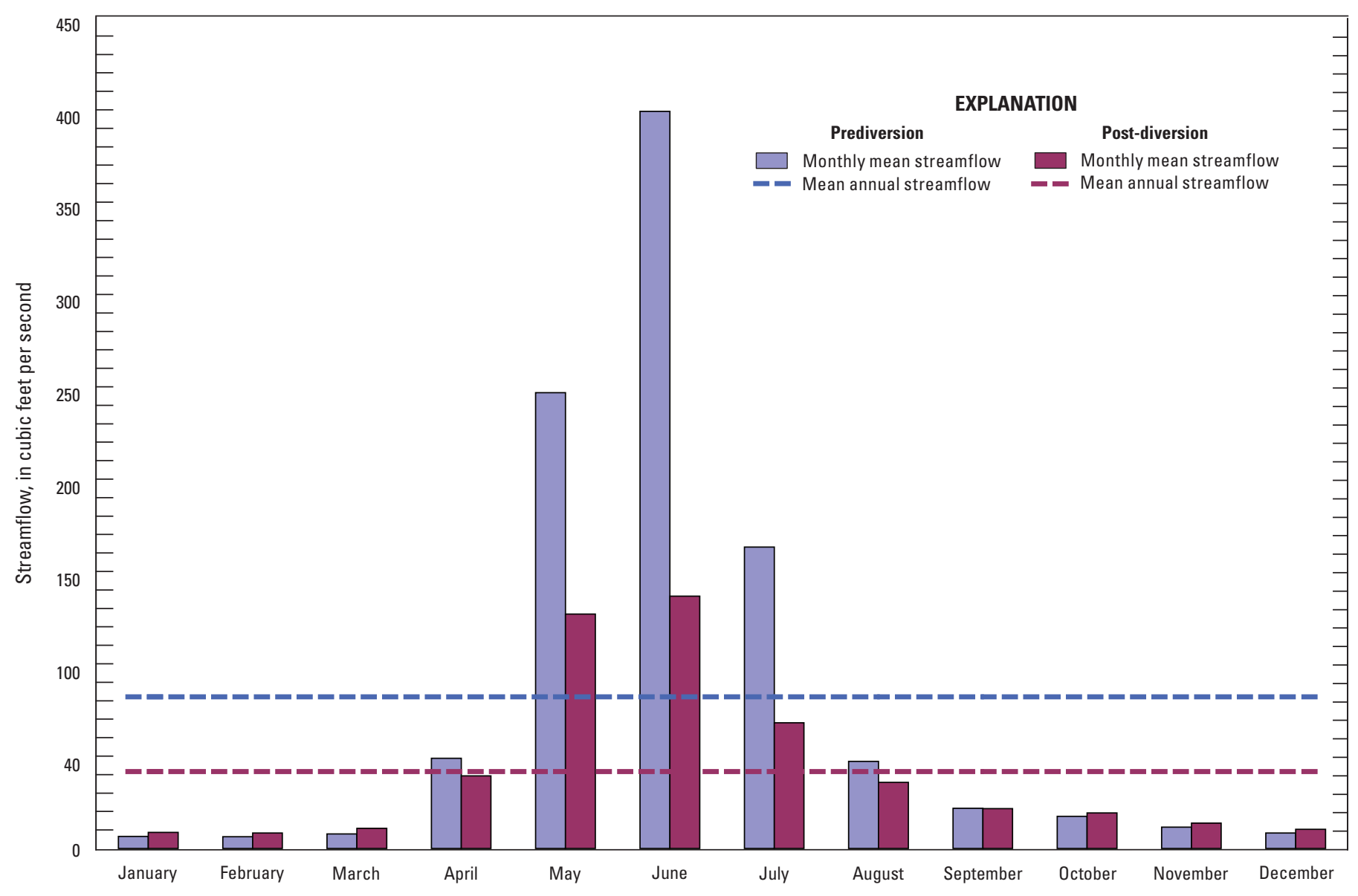

Figure 16. Comparison of streamflow prediversion (1945-1965) and post-diversion (1966-2008) from Homestake Reservoir at site 27, Homestake Creek near Red Cliff, Colorado. 


\section{Assessment of Surface-Water Quality}

Natural and human factors can affect surface-water quality. Natural factors affecting water quality include geology, snowpack, soil type, vegetation, topography, streamflow, precipitation, climate, and ecology. Many human effects such as urbanization and agriculture are part of a larger process of watershed-wide land-use or land-cover change that can affect water quality. Understanding the relation between land use and water quality is particularly useful when considering nonpointsource contaminants. Diffuse sources of suspended sediments, pathogens, nutrients, pesticides, emerging contaminants, heavy metals, oil, and road salt often are less visibly obvious than point-source contamination.

In order to pinpoint diffuse sources of contamination, studies often require more intensive monitoring programs and special research efforts. Correlations between land use and water quality often are complex and, in general, are likely to be site or region specific. Understanding the relation between land use and water quality in each watershed is useful for the landuse management specific to that region. The inclusive approach to this study and associated data limitations does not allow for the analysis to convey specific conclusions which identify stressors and their immediate effects within the watershed.

Surface-water-quality data from 293 sites and 12 different source agencies were complied into 192 unique sites located on streams and rivers in the ERW (table 5). Nearly 39 percent (74 sites) of the unique sites had fewer than 5 samples. In comparison, 23 percent (44 sites) of the sites have more than 100 samples. Physical properties were the most abundant type of samples collected, with major ions, nutrients, and trace elements also commonly collected.

As mentioned in "Data Review and Analysis," where adequate data existed, determination of summary statistics, comparisons to State standards and Federal recommendations, and trend analysis was done. Discussion of surface-water quality is presented in the following sections based on constituent groupings: "field properties, dissolved solids and major ion, nutrients, trace elements, and suspended sediment."

\section{Field Properties}

Agriculture, industry, urbanization, and mining all affect field properties. Field properties analyzed for in this study included specific conductance, $\mathrm{pH}$, water temperature, dissolved oxygen, and hardness (table 8). These constituents are indicators of water-quality conditions.

\section{Specific Conductance}

Specific conductance is proportional to the dissolvedsolids concentration within the stream and has the ability to conduct an electric current. The presence of charged ionic species in a solution makes the solution conductive. The weathering of minerals in soil and bedrock contribute as a primary source of major dissolved constituents. The major ions that comprise the total dissolved solids in the water column are presented in "Dissolved solids and Major Ions."

Specific conductance in the ERW was measured at 163 sites from January 12, 1968, to 2007, and ranged from less than 50 to $4,550 \mu \mathrm{S} / \mathrm{cm}$ depending on site and time of year. The 25th, 50th, and 75th percentiles for specific conductance at sites in the ERW were 97, 152, and $250 \mu \mathrm{S} / \mathrm{cm}$, respectively. Median values for the subwatersheds ranged from $105 \mu \mathrm{S} / \mathrm{cm}$ in Gore Creek, $138 \mu \mathrm{S} / \mathrm{cm}$ in the Upper Eagle, $295 \mu \mathrm{S} / \mathrm{cm}$ in the Middle Eagle, to $809 \mu \mathrm{S} / \mathrm{cm}$ in the Lower Eagle (table 8). The spatial pattern shows an increase in specific conductance as water moves downstream, with a defined increase in specific conductance occurring near the mouth of Gore Creek and downstream of Gore Creek. Additionally, there also may be a localized area of increased values within Black Gore Creek near Vail (site 11) and Turkey Creek near Red Cliff (site 15) (fig. 17).

Evaluation of temporal trends was done for all sites with specific conductance data; trend analysis was done at the 8 sites meeting the criteria set in the "Data Sources, Compilation, Review, and Analysis" section of this report. Findings from the trend analysis are presented in table 9 and figure 17. A comparison between sites where trend assessments were completed was done to find areas within the ERW that may be contributing to the observed trends. Seasonal Kendall Trend tests were done based on data availability for 1990-2007. There is a significant downward trend ( $\mathrm{p}$-value of 0.03) in specific conductance (flow-adjusted) at site 59 (Upper Eagle subwatershed), which represents a $47.8-\mu \mathrm{S} / \mathrm{cm}$ decrease over the time period. Upstream at site 37 (Upper Eagle subwatershed) near Gilman there is no flow-adjusted trend for 1990-2007. The area between these sites (59 and 37) may represent the area within the ERW contributing to the observed trend at site 59 and this area contains Fall Creek and Rock Creek (fig 1. and fig. 17).

$\mathrm{pH}$

The $\mathrm{pH}$ is an important property of water that can limit the diversity and quantity of biota of aquatic communities (Allan, 1996) and can be a controlling component of solubility (Stumm and Morgan, 1996). The $\mathrm{pH}$ is calculated as the negative $\log 10$ of hydrogen ions concentration occurring between 0 and 14 (Brown and others, 2006). The $\mathrm{pH}$ is a measure of how acidic or basic a solution is. Acids are represented by values $0-7$ and bases are represented by values 7-14; a value of 7 is neutral. Lower $\mathrm{pH}$ can contribute to increased solubility of metals (Hem, 1989).

The $\mathrm{pH}$ of a stream is affected by biological processes, geology, precipitation, and human activities. Within the ERW, $\mathrm{pH}$ in streams may be altered by minerals within the geologic formations (Tweto, 1979). The Colorado Mineral Belt contains metal ores that when oxidized by water produce acidic water. This process is naturally occurring within the ERW, but may be accelerated within mining areas. Numerous sedimentary 
Table 8. Summary statistics of field properties and suspended-sediment data in the Eagle River watershed by subwatershed, $1947-2007$.

[No., number; --, undetermined; MRL, Minimum Reporting Limit; $\mu \mathrm{S} / \mathrm{cm}$, microsiemens per centimeter at 25 degrees Celsius; ${ }^{\circ} \mathrm{C}$, degrees Celsius; ntu, nephelometric turbidity units; $\mathrm{mg} / \mathrm{L}$, milligrams per liter; $\mathrm{CaCO}_{3}$, calcium carbonate]

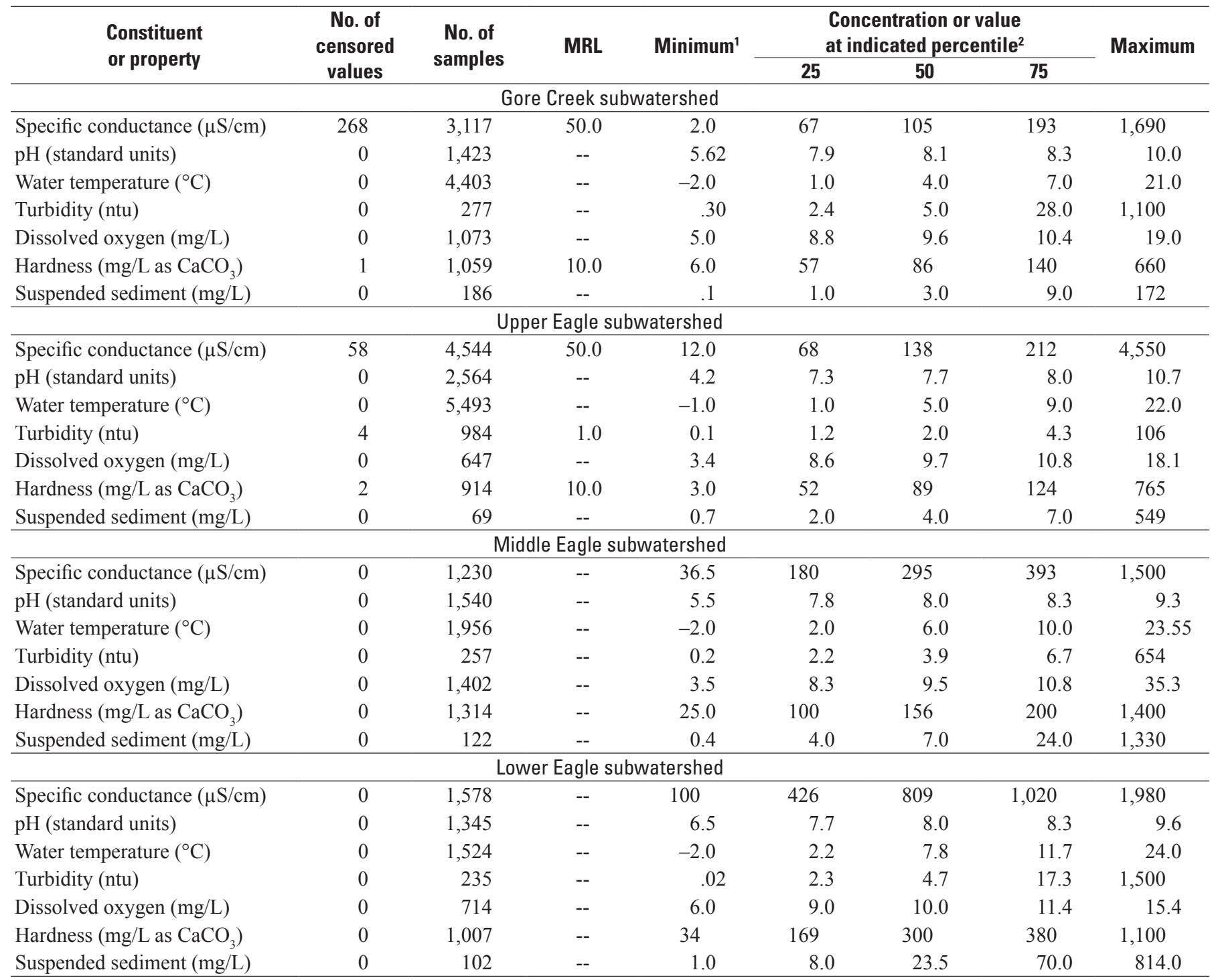

${ }^{1}$ Uncensored values.

${ }^{2}$ Percentile values can be less than the MRL values due to multiple MRLs within the combined dataset. 
Table 9. Summary of monotonic temporal trends for field properties and suspended-sediment concentrations in the Eagle River watershed, Colorado.

[No., number; --, undetermined; $\mu \mathrm{S} / \mathrm{cm}$, microsiemens per centimeter at 25 degrees Celsius; $\mathrm{mg} / \mathrm{L}$, milligrams per liter; $\mathrm{CaCO}_{3}$, calcium carbonate]

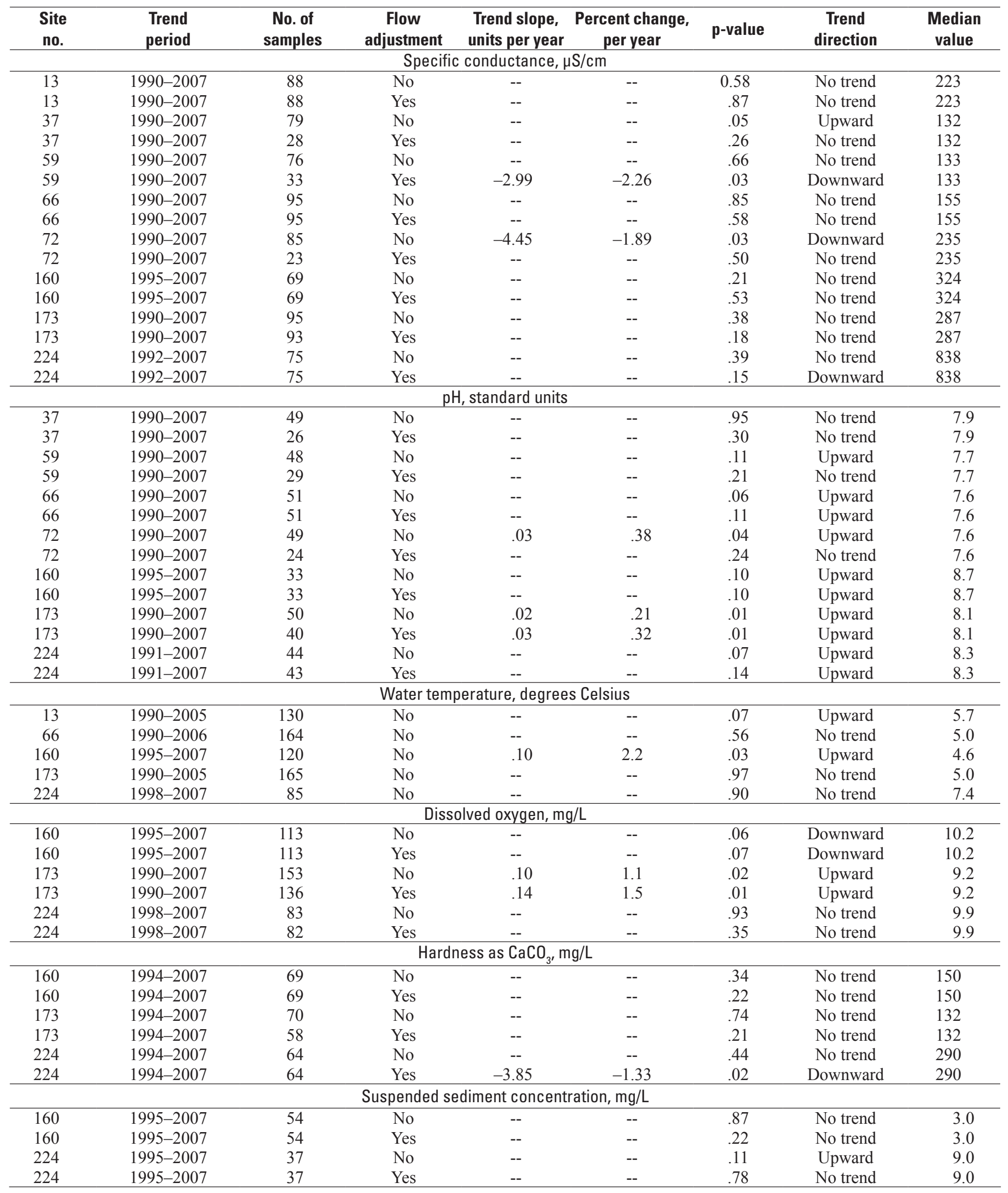




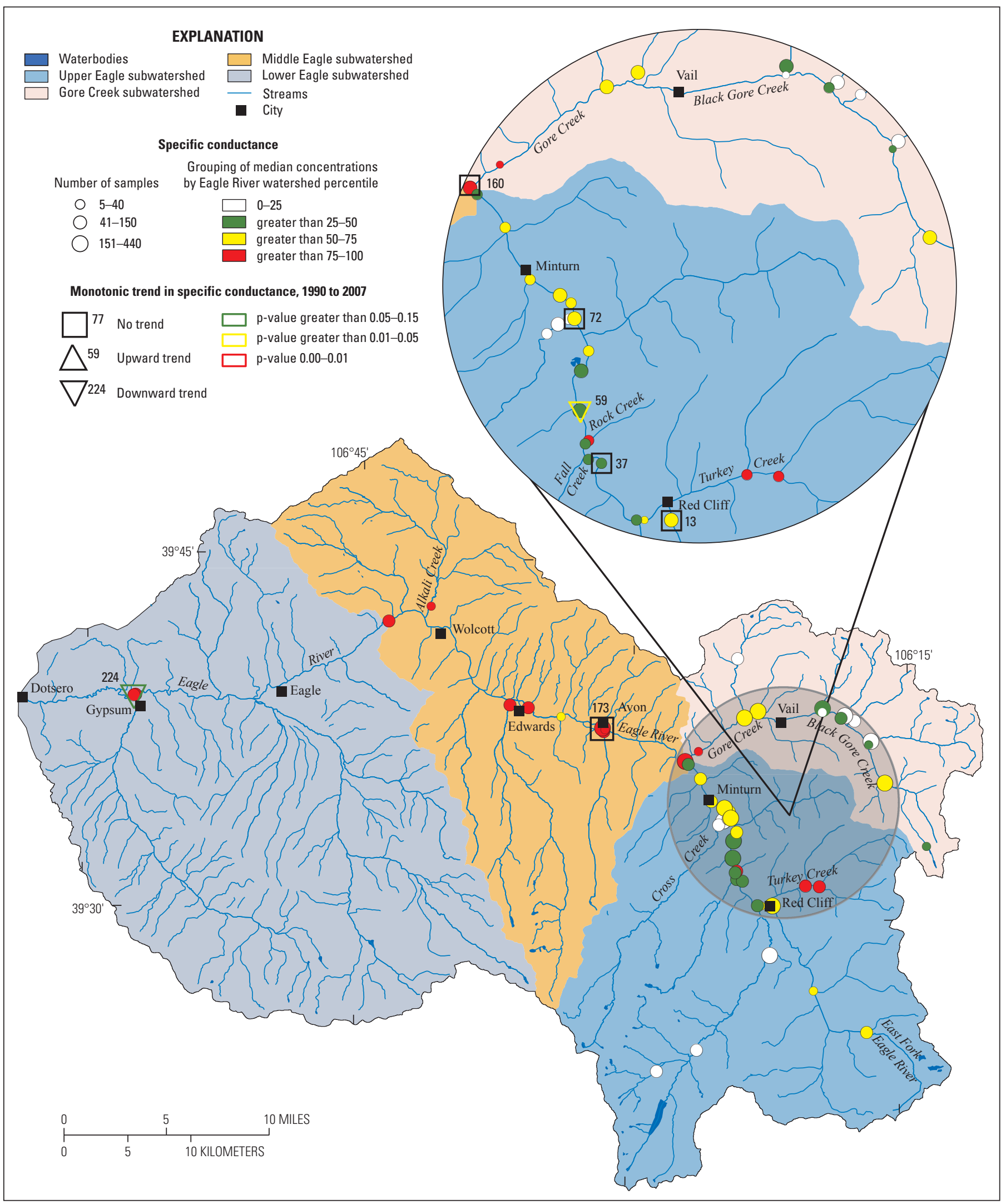

Base from U.S. Geological Survey digital data, 2007,

Universal Transverse Mercator projection

Zone 13

Figure 17. Spatial distribution of median-concentration percentiles and monotonic trends in specific conductance at selected sites in the Eagle River watershed, Colorado, 1990-2007. 
units, including the Minturn Formation and the Belden Formation contain carbonate minerals. When these minerals are dissolved in water, $\mathrm{pH}$ increases. This process also occurs naturally within the ERW but also may be increased through water storage and irrigation within the watershed. If storage or irrigation is occurring in formations containing metal ores, the $\mathrm{pH}$ could decrease.

Seasonal variations in $\mathrm{pH}$ occur in two general patterns within the Eagle River watershed based on how much influence the mining area has on the $\mathrm{pH}$ (fig. 18). In areas heavily affected by mining, $\mathrm{pH}$ tends to be lowest during February and March; the median concentrations are lower than areas less affected by mining. In areas less affected or unaffected by mining, $\mathrm{pH}$ tends to be lowest during peak streamflows during May and June and highest during late fall and early spring.

The $\mathrm{pH}$ in the ERW has been measured at 175 sites from January 12, 1968, to the present (2009), and ranged from 4.2 to 10.7. The 25th, 50th, and 75th percentiles for the ERW were 7.7, 8.0, and 8.2, respectively. Median concentrations for the subwatersheds were 8.1 in Gore Creek, 7.7 in the Upper Eagle, 8.0 in the Middle Eagle, and 8.0 in the Lower Eagle (table 8). The spatial pattern shows increase in $\mathrm{pH}$ as water moves downstream, both within the subwatersheds and the watershed as a whole; with the exception of the main stem Eagle River near areas subject to acid-mine drainage from several tributaries in mining areas near Belden (fig. 19). In the mining area, many locations show lower $\mathrm{pH}$ values, with the lowest median concentration occurring near the mouths of Cross and Rock Creeks (7.2 and 7.1, respectively). Additionally, there also may be two localized areas of higher $\mathrm{pH}$ values, within Black Gore Creek near Vail and the East Fork of the Eagle River near Red Cliff.

State standards for $\mathrm{pH}$ are set as a range of acceptable values between 6.5 and 9.0 (Colorado Department of Public Health and Environment, 2008). Measurements of $\mathrm{pH}$ in streams with values outside this range are considered exceedances of the State standard and may indicate possible waterquality concerns. The minimum $\mathrm{pH}$ standard of 6.5 units was exceeded less than 10 times over a period of several years for most sites in the ERW (fig. 20); approximately 1 percent (86 comparisons) of the total number of measurements $(6,872)$ exceeded the minimum $\mathrm{pH}$ standard (table 10). The maximum $\mathrm{pH}$ standard of 9.0 units was exceeded less than 5 times over a period of several years for most sites in the ERW; approximately 1 percent ( 73 comparisons) of the total number of measurements $(6,872)$ exceeded the maximum standard (table 10).

Evaluation of temporal trends was done for all sites with $\mathrm{pH}$ data; trend analysis was done at the 7 sites that met the minimum criteria set in the "Data Sources, Compilation, Review, and Analysis" section of this report. Findings from the trend analysis are presented in table 9 and figure 19. A comparison between sites where trend assessments were completed was done to find areas within the ERW that may be contributing to the observed trends. Seasonal Kendall Trend tests were done based on data availability for 1990-2007. There is a weakly significant flow-adjusted upward trend ( $\mathrm{p}$-value of 0.11) at site 66 (Upper Eagle subwatershed) (table 9). Upstream a short distance at site 59 (Upper Eagle subwatershed), no flow-adjusted trend was detected. The area between these sites may be contributing to the detected trend at site 66, and includes the areas located downstream of the Belden mining area. No other trends exist upstream of this area. Downstream near Avon, a highly significant upward trend ( $\mathrm{p}$-value of 0.01) exists at site 173 (Middle Eagle subwatershed). This trend represents an increase of 0.5 standard $\mathrm{pH}$ units over the entire specified time period. A flow-adjusted upward trend is detected as weakly significant (p-value of 0.14 ) at a site downstream near Gypsum (site 224, Lower Eagle subwatershed). No flow-adjusted trends were detected at site 72 (Upper Eagle subwatershed), which limits the potential area contributing to the trend detected at site 173 (Middle Eagle subwatershed) to a large area of the ERW as shown in figure 19.

\section{Water Temperature}

Temperature is an important physical property of water that can affect aquatic biota and stream chemistry. Water temperature affects larval maturation rates and other life cycles of biota in aquatic habitats (Allan, 1996). The rate of many chemical reactions is related proportionally to temperature. For example, as temperature increases, the rates of reaction also can increase. Water temperature often is related to ambient-air temperature, but also is affected by the source of the water (groundwater, lake, or snow melt), the geomorphology of the stream channel, and various human effects.

Water temperature in the ERW has been measured at 181 sites from April 30, 1958, to the present (2009), and ranged from -2 to 24 degrees Celsius (table 8). The 25th, 50th, and 75th percentiles for the ERW were 1.4, 4.9, and 8.9 degrees Celsius, respectively. Summary statistics for temperature are shown in table 8. Median temperatures for the ERW generally increase in the downstream direction as evidenced by the subwatershed median temperatures (50th percentile) that ranged from 4.0 degrees Celsius in Gore Creek, 5.0 degrees Celsius in the Upper Eagle, 6.0 degrees Celsius in the Middle Eagle, to 7.8 degrees Celsius in the Lower Eagle (fig 21). The highest median temperature observed in the ERW occurred near Gypsum (9.0 degrees Celsius). Seasonal variations in the median temperatures show that water temperatures tend to be coldest December-February and warmest July-August following ambient air-temperature patterns (fig 22). The greatest rates of temperature change occur as an increase between June-July and as a decrease between September-October (fig. 22).

State standards for water temperature (chronic) are specific to CDPHE Regulation 33 (Colorado Department of Public Health and Environment, 2007). The water-temperature State standard was exceeded less than 10 times over a period of several years for most sites in the ERW; less than 1 percent (89 comparisons) of the total number of measurements $(13,376)$ exceed the State standard for chronic water temperature (table 10 and fig 23). 


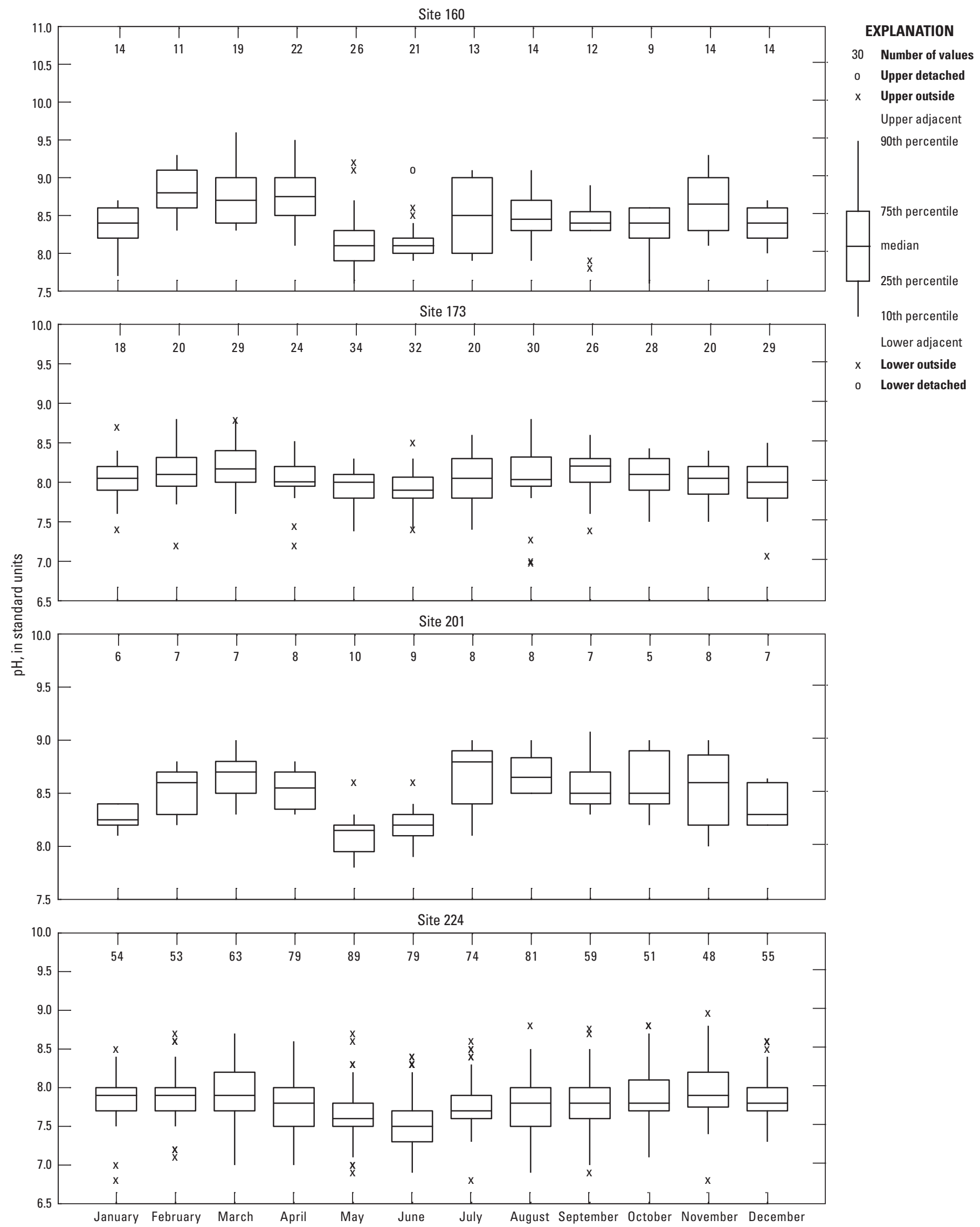

Figure 18. Seasonal pattern of pH at selected sites in the Eagle River watershed, Colorado, 1947-2007. 


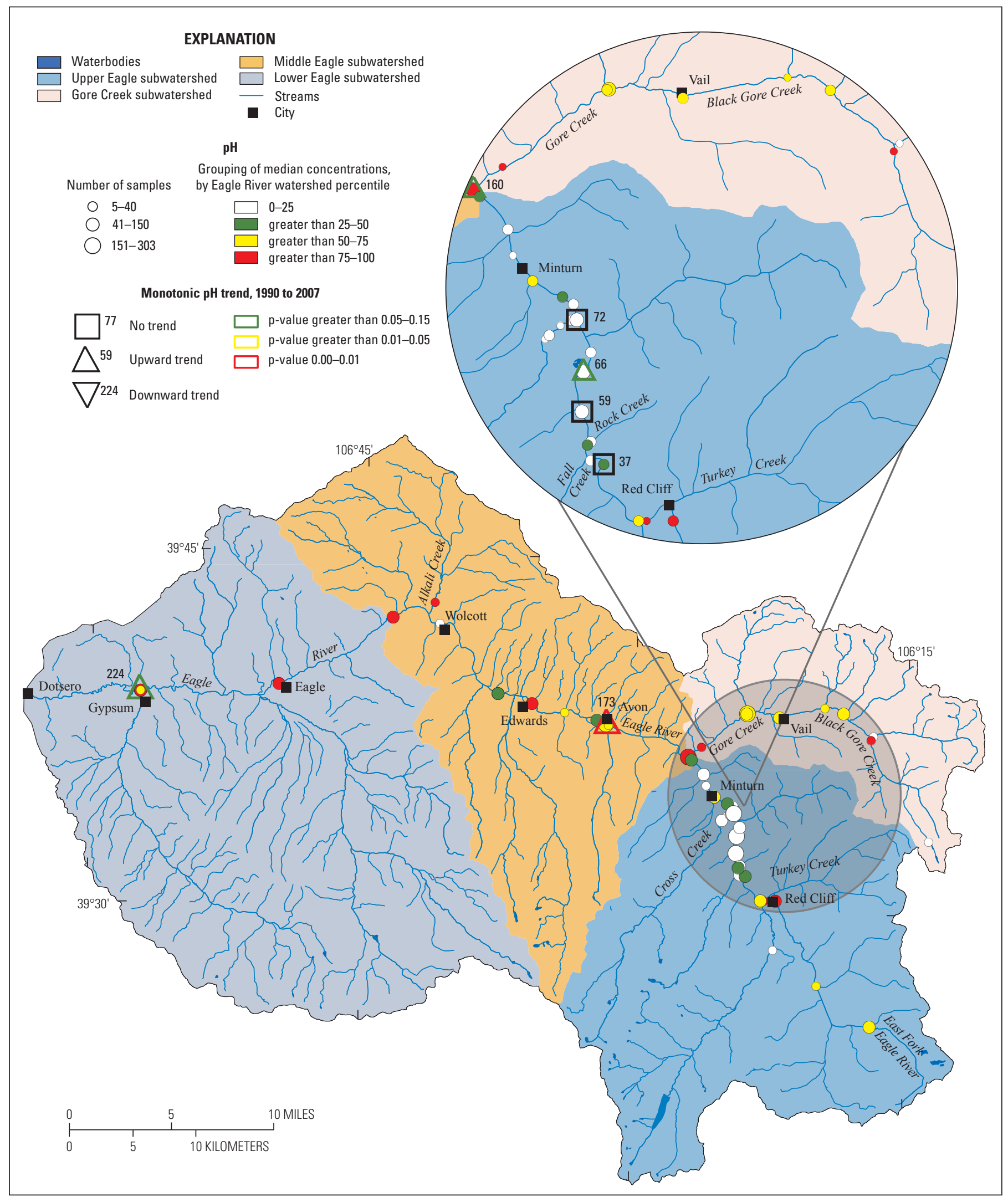

Base from U.S. Geological Survey digital data, 2007,

Universal Transverse Mercator projection

Zone 13

Figure 19. Spatial distribution of median-concentration percentiles and monotonic pH trends at selected sites in the Eagle River watershed, Colorado, 1990-2007. 
Table 10. Summary of Colorado exceedances of water-quality standards for field properties in the Eagle River watershed, Colorado by subwatershed.

[No., number; --, no data; <, less than; mg/L, milligrams per liter; $\mu \mathrm{g} / \mathrm{L}$, micrograms per liter; ${ }^{\circ} \mathrm{C}$, degrees Celsius]

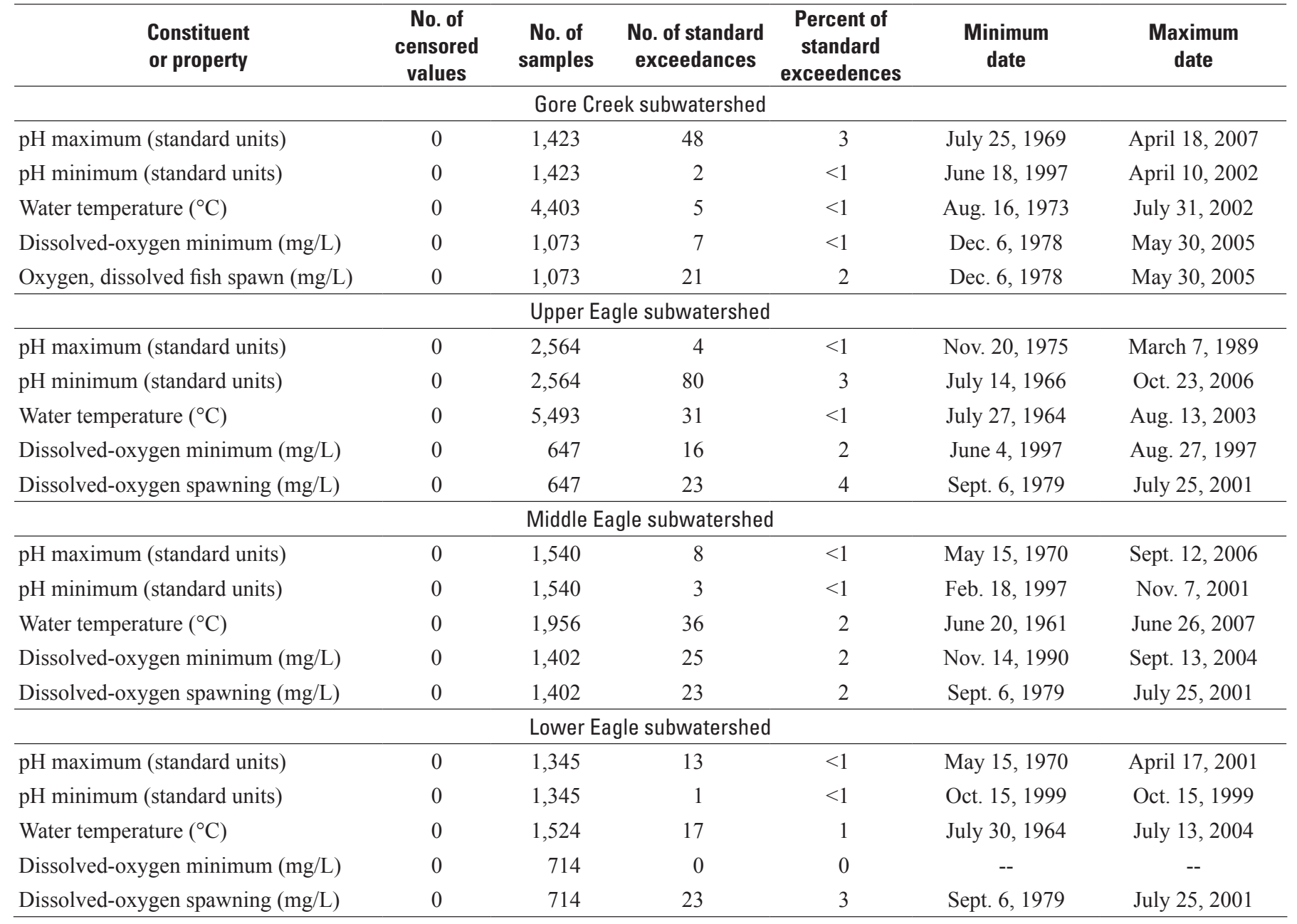




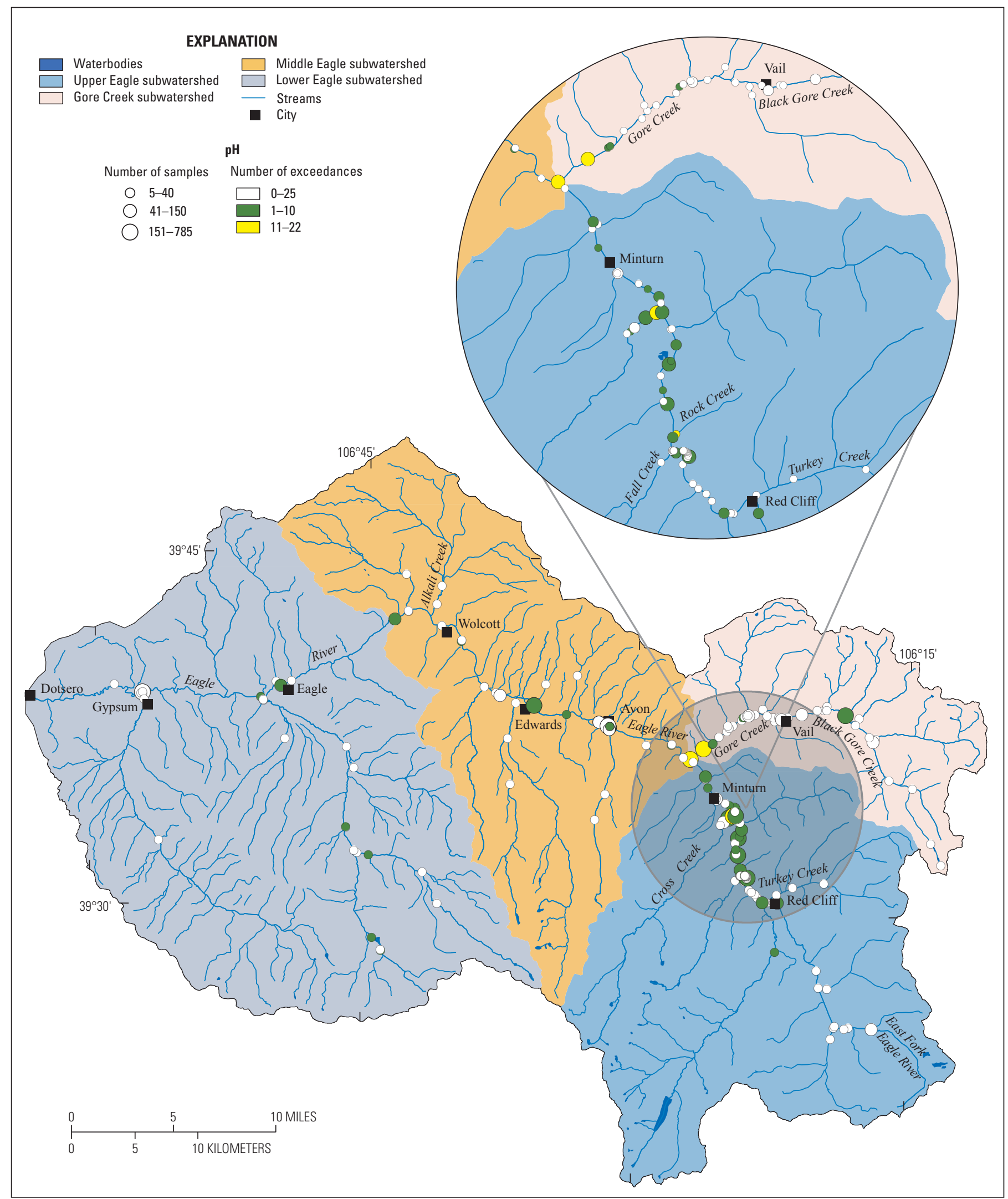

Base from U.S. Geological Survey digital data, 2007,

Universal Transverse Mercator projection

Zone 13

Figure 20. Number of exceedances of the Colorado water-quality standard for $\mathrm{pH}$ at selected sites in the Eagle River watershed, Colorado, 1947-2007. 


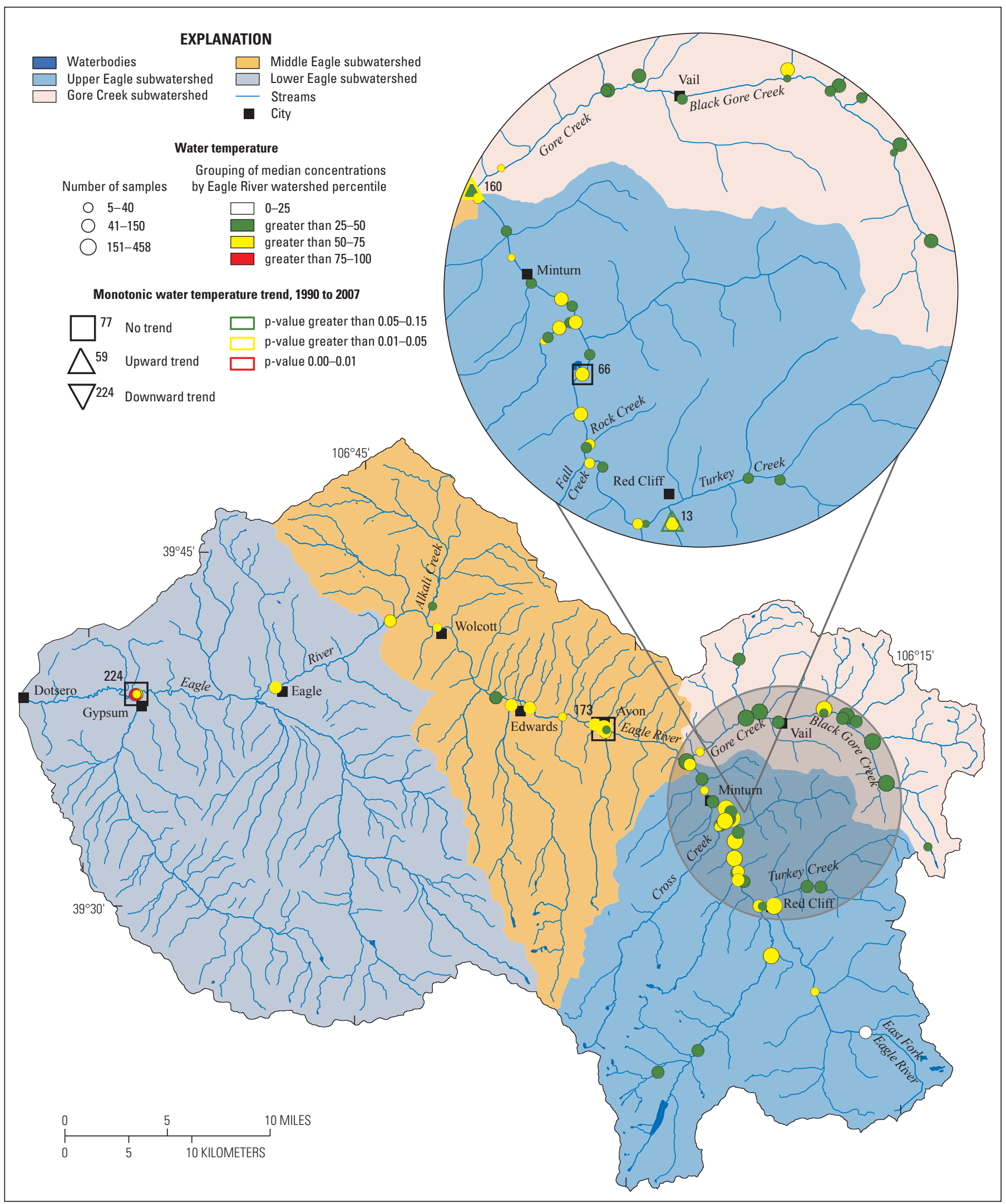

Base from U.S. Geological Survey digital data, 2007,

Universal Transverse Mercator projection

Zone 13

Figure 21. Spatial distribution of median-concentration percentiles and monotonic water temperature trends at selected sites in the Eagle River watershed, Colorado, 1990-2007. 

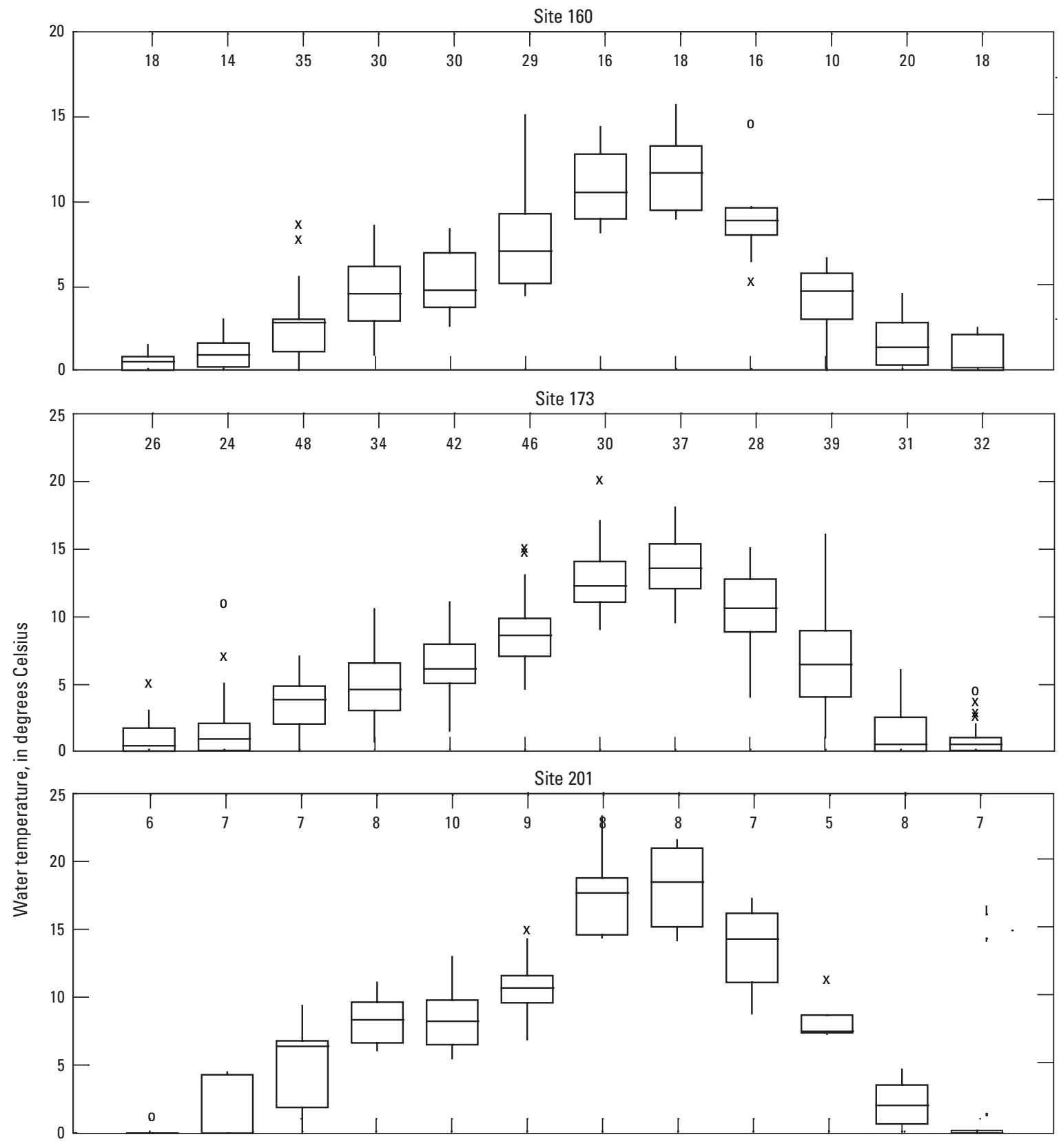

\section{EXPLANATION}

30 Number of values

o Upper detached

$x \quad$ Upper outside

Upper adjacent

90th percentile

75th percentile

median

25th percentile

10th percentile

Lower adjacent

Lower outside

o Lower detached

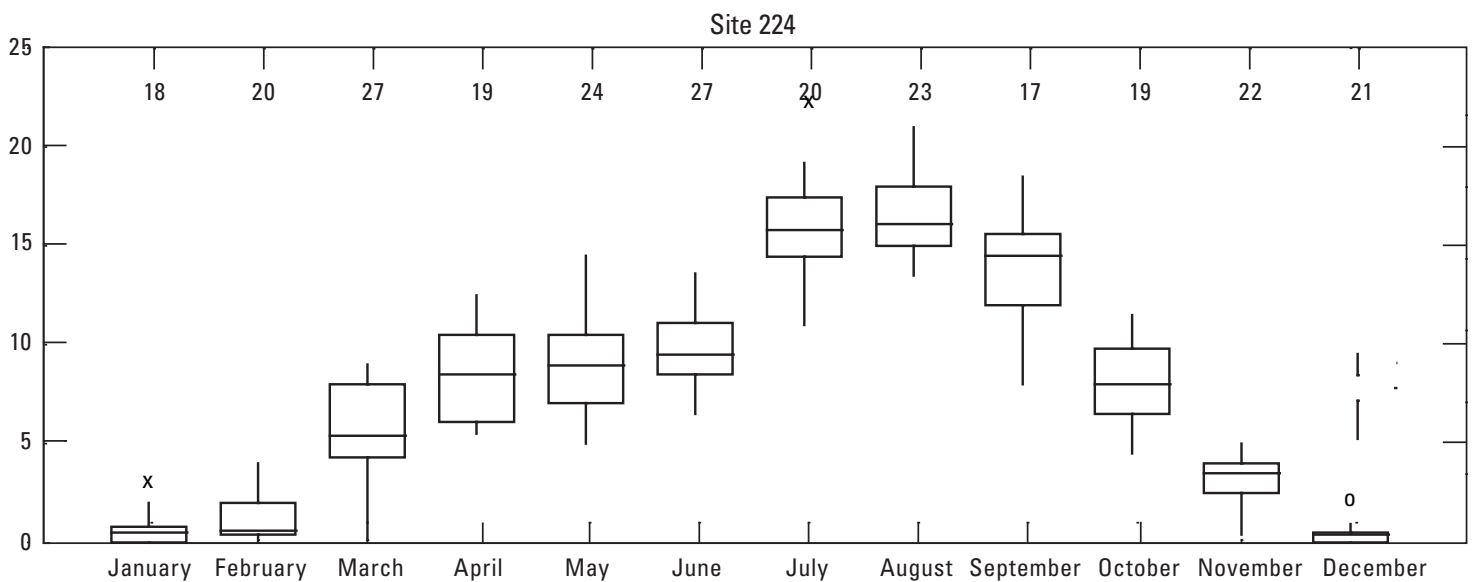

Figure 22. Seasonal pattern of water temperature at selected sites in the Eagle River watershed, Colorado, 1947-2007. 


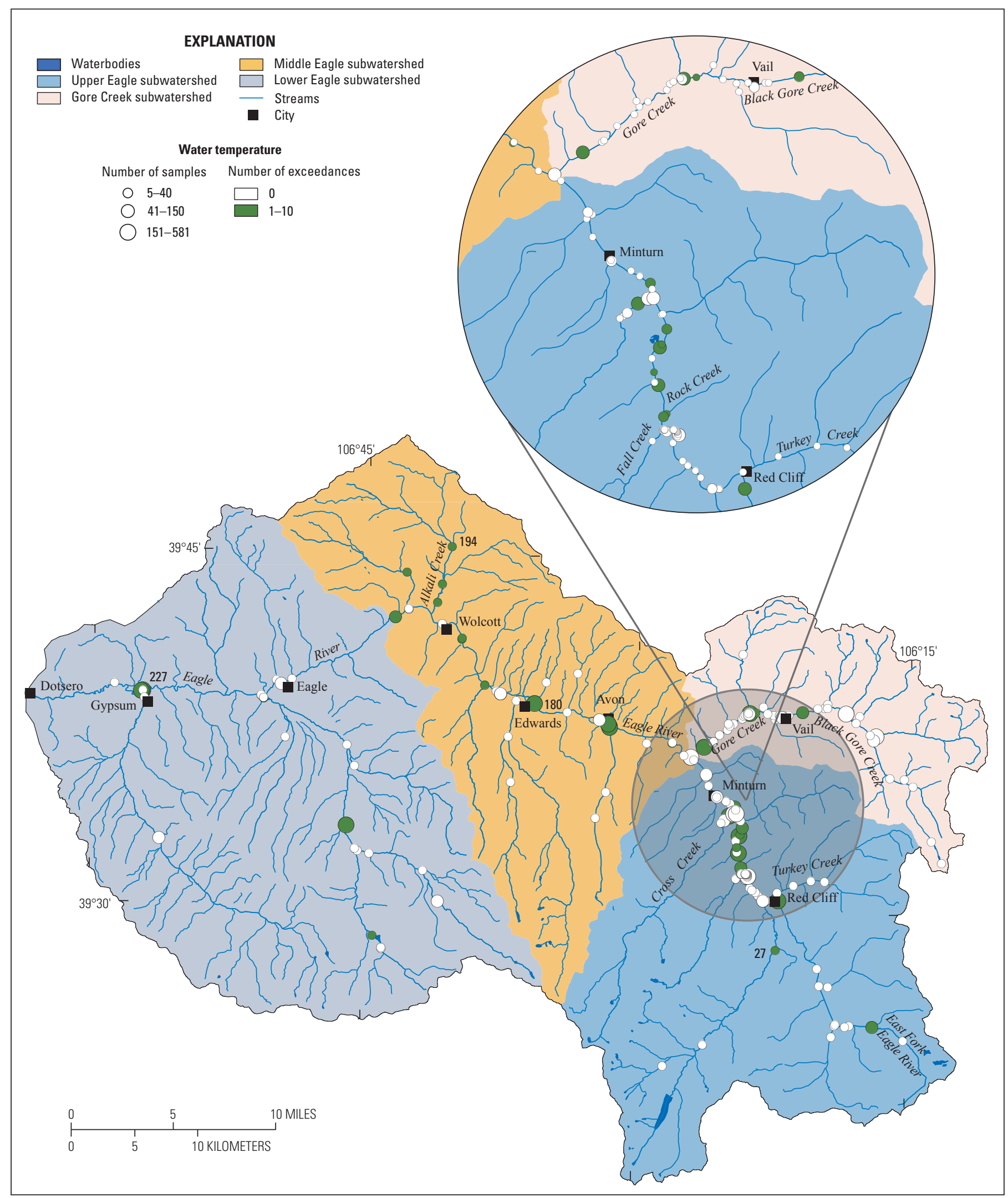

Base from U.S. Geological Survey digital data, 2007,

Universal Transverse Mercator projection

Zone 13

Figure 23. Number of exceedances of the Colorado water-quality standard for water temperature at selected sites in the Eagle River watershed, Colorado, 1947-2007. 
Evaluation of temporal trends was done for all sites with water temperature data; trend analysis was done at the 5 sites that met the criteria set in the "Data Sources, Compilation, Review, and Analysis" section of this report. Findings from trend analysis are presented in table 9 and figure 21 . A comparison between sites where trend assessments were completed was done to locate areas within the ERW that may be contributing to the observed trends. Upward trends at sites 13 (Upper Eagle subwatershed) (p-value of 0.07) and 160 (Gore Creek subwatershed) (p-value of 0.03) indicate increasing water temperatures at (1) the Eagle River above Red Cliff and (2) in Gore Creek. The trend on Gore Creek (table 9) is equivalent to a 1.3 degrees Celsius change over the entire specified time period (1995-2007).

\section{Dissolved Oxygen}

Dissolved oxygen is an important property of water that can affect both aquatic biota and chemical reactions within streams. Dissolved oxygen is important for all respiratory functions of aquatic life including both plant and animal species. Dissolved-oxygen levels are important components of oxidation-reduction reactions (redox) and can be important to oxidation states of transition metals like copper, iron, manganese, and zinc (Stumm and Morgan, 1996). Iron and manganese are the predominant participants in redox processes. In the presence of oxygen, iron and manganese are stable only as solid oxides. Soluble forms are present at concentrations less than $10^{-9}$ molar (Stumm and Morgan, 1996). The oxidation state of transition metals is important in determining solubility and mobility of these compounds in natural waters especially downstream of mining areas in the watershed.

The concentration of dissolved oxygen within a stream is dependent on many processes. Dissolved oxygen enters a stream through reactions between the stream and the atmosphere along the surface of the water, and through mechanical mixing of air and water in turbulent areas. Dissolved oxygen also can be introduced directly by aquatic plants during photosynthesis. Contrarily, dissolved oxygen can be consumed and therefore reduced as organic matter dies and decomposes. This important process relates primarily to algal blooms. Dissolvedoxygen concentrations are inversely related to water temperatures; as temperatures increase the capacity of water to hold a given quantity of dissolved oxygen is reduced (Hem, 1989).

Dissolved oxygen-concentration in the ERW has been measured at 121 sites from March 15, 1973, to the present (2009), and ranged from 3.4 to $35.3 \mathrm{mg} / \mathrm{L}$ shown in table 8 . The 25th, 50th, and 75th percentiles for the watershed were $8.4,9.5$, and $10.5 \mathrm{mg} / \mathrm{L}$ respectively. Median concentrations for the subwatersheds ranged from $9.6 \mathrm{mg} / \mathrm{L}$ in Gore Creek, $9.7 \mathrm{mg} / \mathrm{L}$ in the Upper Eagle, $9.5 \mathrm{mg} / \mathrm{L}$ in the Middle Eagle, to $10.0 \mathrm{mg} / \mathrm{L}$ in the Lower Eagle (table 8 ). The spatial pattern is variable, with the highest median concentration occurring in the Eagle River around Edwards (10.9 mg/L) (fig. 24). Seasonal variations in dissolved-oxygen concentrations show that concentrations tend to be lowest from July-September and highest from November-March, which is the inverse of patterns in water temperature (fig. 25).

State standards for dissolved oxygen (chronic) are set for both minimum dissolved oxygen $(6.0 \mathrm{mg} / \mathrm{L})$ and minimum dissolved oxygen for fish spawning $(7.0 \mathrm{mg} / \mathrm{L})$ (Colorado Department of Public Health and Environment, 2007). The minimum dissolved oxygen State standard was exceeded less than 10 times over a period of several years for most sites in the ERW; approximately 1 percent (48 comparisons) of the total number of measurements $(3,836)$ exceeded the State standard $(6.0 \mathrm{mg} / \mathrm{L})$ for chronic dissolved oxygen (table 10 and fig 26). Sites exceeding the minimum dissolved oxygen (spawning) State standard $(7.0 \mathrm{mg} / \mathrm{L})$ typically have less than 10 exceedances over a period spanning several years for most sites in the ERW; approximately 3 percent (90 comparisons) of the total number of measurements $(3,836)$ exceeded the dissolved oxygen (spawning) State standard (table 10).

Evaluation of temporal trends was done for all sites with dissolved oxygen data; trend analysis was done at the 3 sites that met the minimum criteria set in the "Data Sources, Compilation, Review, and Analysis" section of this report. Findings from the trend analysis are presented in table 9 and figure 24. A comparison between sites where trend assessments were completed was done to find areas within the ERW that may be contributing to the observed trends. A weakly significant flow-adjusted downward trend ( $\mathrm{p}$-value less than 0.07) at site 160 (Gore Creek subwatershed) (1995-2007) was detected. A highly significant flow-adjusted upward trend (p-value less than 0.01) at site 173 (Middle Eagle subwatershed) (1990-2007) was detected. No trend was detected at site 224 (Lower Eagle subwatershed). No other sites were available to compare to this trend for the same time period, so it is not possible to limit the area contributing to the trend. The upward trend represents a $2.5-\mathrm{mg} / \mathrm{L}$ change over the entire specified time period.

\section{Hardness}

Hardness is an important property of water because it can be used to calculate the toxicity of metals in aquatic environments, and it also is an important consideration for municipalwater supply purposes. Hardness can be important to aquatic life because it can have an effect on the toxicity of metals; as hardness increases, trace-metal toxicity can decrease (Santore and others, 2001). For municipal water suppliers, the term "hard water" is used to describe the reaction of water with soaps and detergents to produce insoluble soap scum or the scale left when water is heated. Although hardness can have many definitions, in this analysis it will be interpreted as a measure of calcium and magnesium abundance (Hem, 1989).

Hardness within the ERW is likely related to the geology (Tweto, 1979). Geologic units rich in calcium, magnesium, other alkaline earth metals, and some heavy metals, can contribute to increases in hardness (Hem, 1989). In the ERW, sedimentary formations containing carbonates, such as the Minturn Formation and the Belden Formation, as well as 


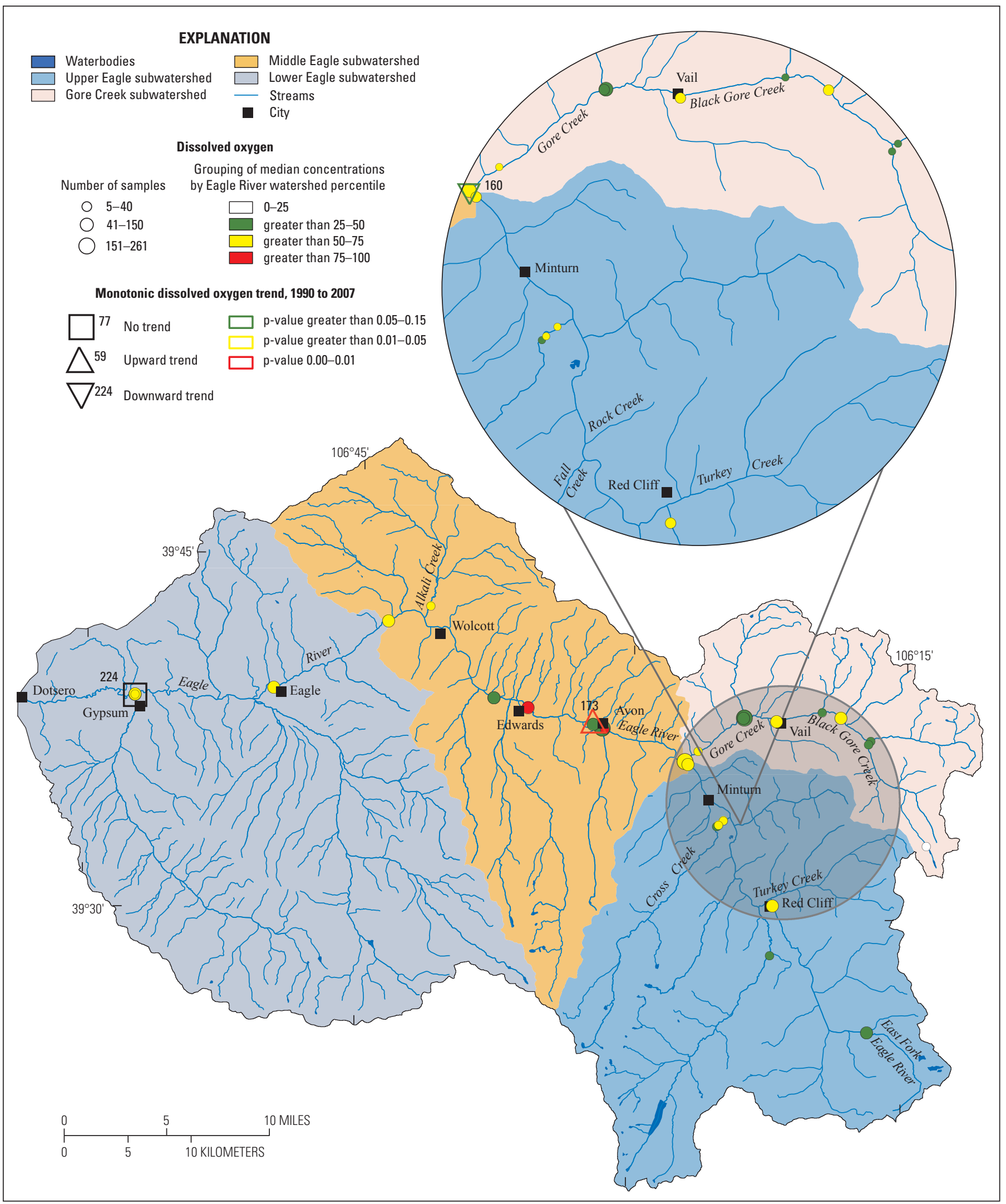

Base from U.S. Geological Survey digital data, 2007,

Universal Transverse Mercator projection

Zone 13

Figure 24. Spatial distribution of median-concentration percentiles and monotonic dissolved-oxygen concentration trends at selected sites in the Eagle River watershed, Colorado, 1990-2007. 


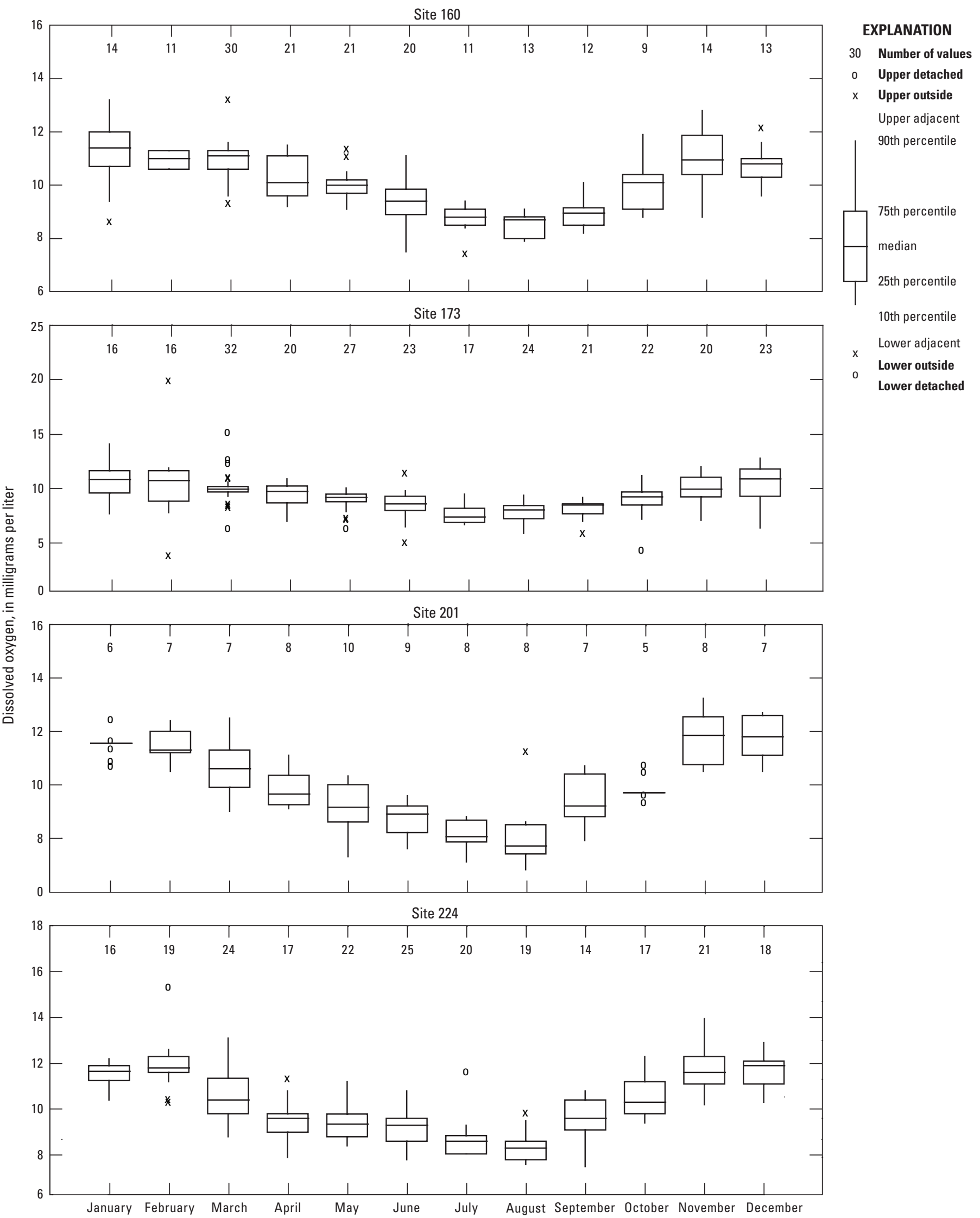

Figure 25. Seasonal pattern of dissolved oxygen at selected sites in the Eagle River watershed, Colorado, 1947-2007. 


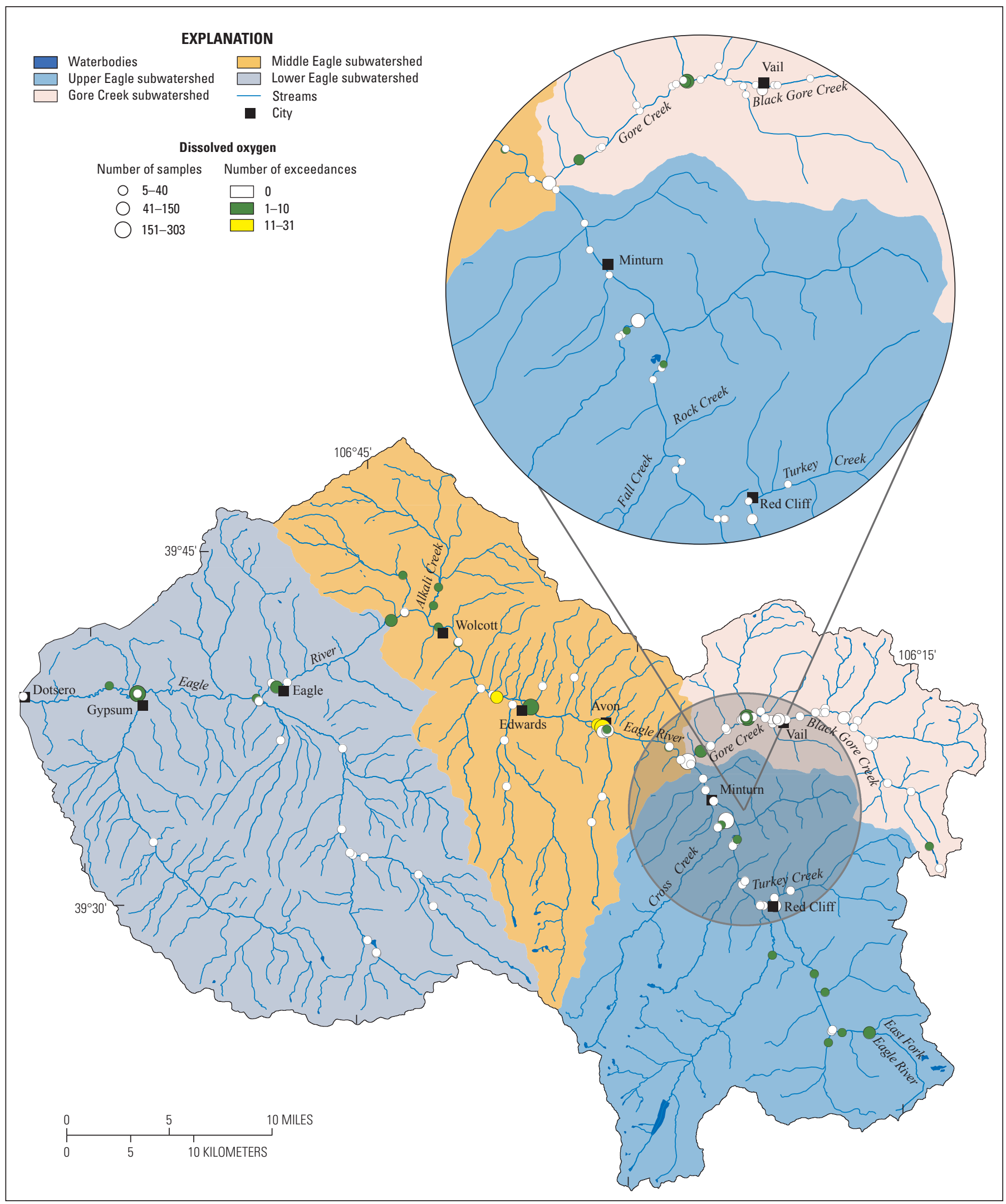

Base from U.S. Geological Survey digital data, 2007,

Universal Transverse Mercator projection

Zone 13

Figure 26. Number of exceedances of the Colorado water-quality standard for dissolved oxygen at selected sites in the Eagle River watershed, Colorado, 1947-2007. 
sandstones or shales containing carbonate cement may contribute to increases in hardness (Tweto, 1979). The Eagle Valley Evaporite may also be a source of calcium from the minerals gypsum and anhydrite. Human effects on hardness may result from water storage (reservoirs), which can cause increases in hardness through evapoconcentration and increased infiltration of water into the surrounding geologic units; municipal-watertreatment operations, which use additives such as carbonates in the treatment process; agriculture soil amendments and fertilizers; and winter road maintenance.

Hardness in the ERW has been measured at 116 sites from 1970 to 2009, and ranged from less than 10 to $1,400 \mathrm{mg} / \mathrm{L}$ shown in table 8 . The $25 \mathrm{th}, 50 \mathrm{th}$, and 75 th percentiles for the ERW were 76, 130, and $188 \mathrm{mg} / \mathrm{L}$, respectively. Median concentrations for the subwatersheds ranged from $86 \mathrm{mg} / \mathrm{L}$ in Gore Creek, $89 \mathrm{mg} / \mathrm{L}$ in the Upper Eagle, $156 \mathrm{mg} / \mathrm{L}$ in the Middle Eagle, to $300 \mathrm{mg} / \mathrm{L}$ in the Lower Eagle (table 8). The spatial pattern shows an increase in hardness concentration as water moves downstream, with a defined increase in hardness in areas draining the Eagle Valley Evaporite and downstream of Edwards. Additionally, there also may be a localized area of higher concentrations within Black Gore Creek near Vail (fig. 27). Seasonal variations in hardness show that concentrations tend to be lowest May-June and highest January-March which is the inverse of patterns in streamflow (fig. 28). Snowmelt derived waters tend to have lower hardness concentrations compared to waters derived from groundwater sources. The greatest rate of hardness concentration change occurs as an increase July-August and as a decrease March-May.

Evaluation of temporal trends was done for all sites with hardness data; trend analysis was done at the 3 sites that met the criteria set in the "Data Sources, Compilation, Review, and Analysis" section of this report. Findings from the trend analysis are presented in table 9 and figure 27. A comparison between sites where trend assessments were completed was done to find areas within the ERW that may be contributing to the observed trends. Seasonal Kendall Trend tests were done for the time period 1994-2007. There is a significant downward trend (p-value of 0.02) in hardness at site 224 (Lower Eagle subwatershed). This trend represents a 53.9-mg/L decrease over the entire specified time period. Upstream at site 173 (Middle Eagle subwatershed) near Avon there is no trend. The area between these sites may represent the area within the ERW contributing to the observed trend.

\section{Dissolved Solids and Major lons}

Dissolved solids (TDS) typically are measured using two different analytical methods. When dissolved solids are calculated through the sum of concentrations of commonly occurring major ions (calcium, magnesium, sodium, potassium, alkalinity, sulfate, chloride, fluoride, and silica) within a water-quality sample, this method is referred to as sum of constituents (SOC). Measures of the solids or salt remaining after evaporation of a water sample is another common method for the determination of dissolved solids in water samples; this method is referred to as residue on evaporation (ROE) (Hem, 1989).

Higher concentrations of dissolved solids can reduce the value of water for agricultural uses and human consumption. The relative percentages of the major ion concentrations in a water sample can be used to classify the water type and can help identify water sources (Freeze and Cherry, 1979). Understanding the source of a water sample can provide insight into important hydrologic processes within a watershed. This can provide useful ancillary data for consideration in the interpretation of water-quality findings. The major ions dissolved in most natural waters typically include calcium, magnesium, sodium, potassium, bicarbonate, carbonate, sulfate, chloride, fluoride, and silicate.

Although complete major-ion data have not been routinely collected at many sites within the watershed, TDS (SOC method) in the ERW has been measured at 72 sites from April 1, 1947, to September 30, 2007, and ranged from 20 to $1,680 \mathrm{mg} / \mathrm{L}$. The $25 \mathrm{th}, 50 \mathrm{th}$, and 75 th percentiles for the ERW were 95, 159, and $290 \mathrm{mg} / \mathrm{L}$, respectively). Median values for the subwatersheds ranged from $130 \mathrm{mg} / \mathrm{L}$ in Gore Creek, $120 \mathrm{mg} / \mathrm{L}$ in the Upper Eagle, $191 \mathrm{mg} / \mathrm{L}$ in the Middle Eagle, to $526 \mathrm{mg} / \mathrm{L}$ in the Lower Eagle (table 11). TDS (ROE method) data are available at additional sites in the ERW that do not have SOC samples. ROE and SOC provide comparable measurements of the TDS concentrations within a water sample. ROE has been measured at 73 sites in the ERW from April 1, 1947, to September 30, 2007. Concentrations ranged from 8 to $1,410 \mathrm{mg} / \mathrm{L}$ throughout the watershed. The $25 \mathrm{th}$, 50th, and 75th percentiles for the ERW were 110, 190, and $310 \mathrm{mg} / \mathrm{L}$, respectively. Median values for the subwatersheds ranged from $92 \mathrm{mg} / \mathrm{L}$ in Gore Creek, $110 \mathrm{mg} / \mathrm{L}$ in the Upper Eagle, $200 \mathrm{mg} / \mathrm{L}$ in the Middle Eagle, to $553 \mathrm{mg} / \mathrm{L}$ in the Lower Eagle (table 11). The spatial patterns for TDS concentrations (SOC method) show increasing concentrations from upstream to downstream with a defined increase occurring near Wolcott. Higher concentrations near the mouth of Gore Creek appear to be diluted by streamflow from the upper Eagle River. Differences in concentrations likely result from increased abundance of salt-bearing geologic formations, such as the Eagle Valley Evaporite, downstream from Avon (Tweto, 1979). Seasonal variations for TDS (SOC method) show that concentrations tend to be lowest May-June and highest October-March, which is the inverse of patterns in streamflow (fig. 30).

Evaluation of temporal trends was done for all sites with TDS (SOC method) data; trend analysis was done at the 4 sites that met the criteria set in the "Data Sources, Compilation, Review, and Analysis" section of this report. Findings from the trend analysis are presented in table 12 and figure 29. A comparison between sites where trend assessments were completed was done to find areas within the ERW that may be contributing to the observed trends. A significant flow-adjusted upward trend (p-value of 0.03) at site 160 (Gore Creek subwatershed 19952007) shows increasing TDS concentrations in Gore Creek. This trend represents a $34.3-\mathrm{mg} / \mathrm{L}$ change over the entire specified time period. A weakly significant flow-adjusted downward trend 


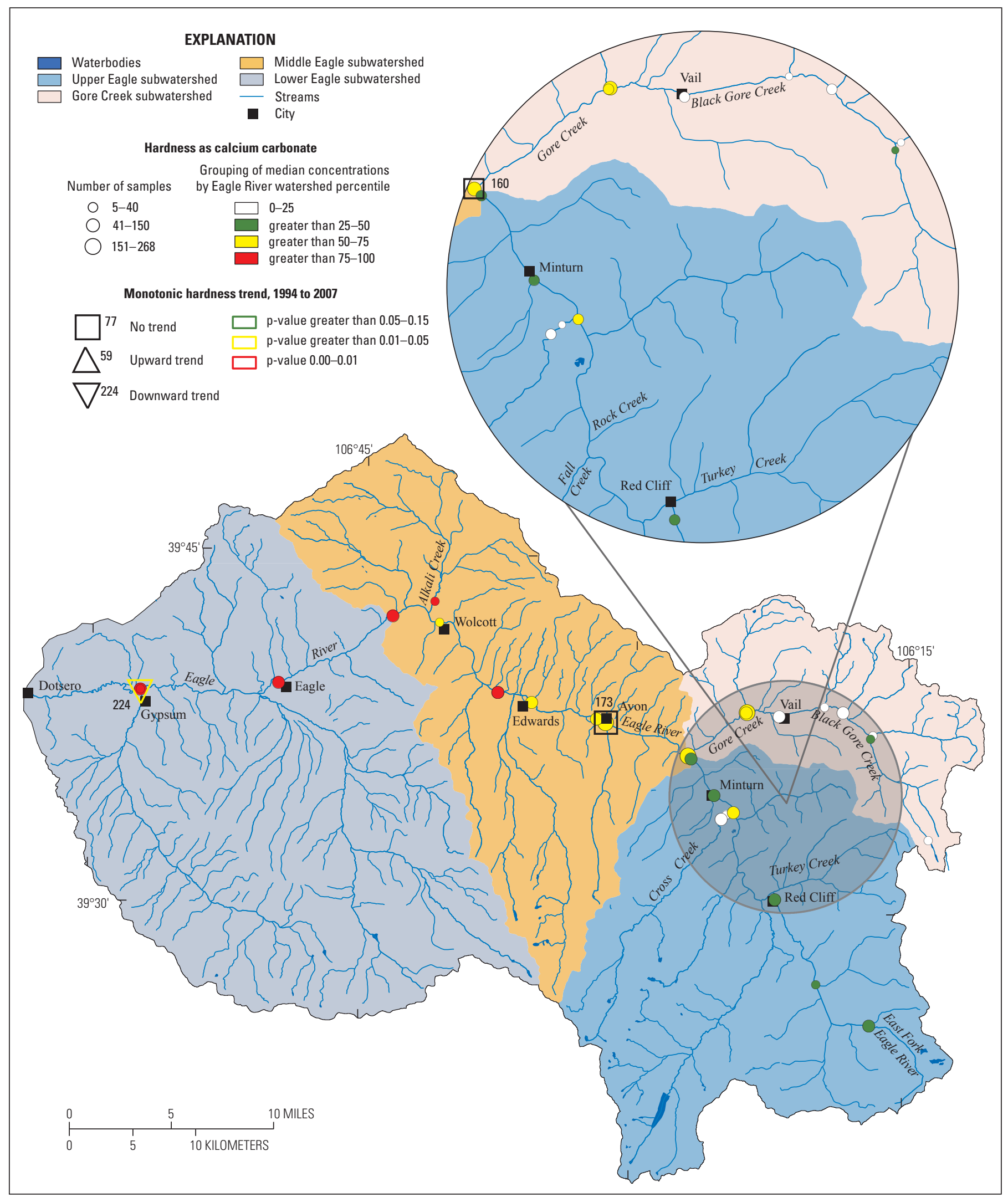

Base from U.S. Geological Survey digital data, 2007,

Universal Transverse Mercator projection

Zone 13

Figure 27. Spatial distribution of median-concentration percentiles and monotonic hardness concentration trends at selected sites in the Eagle River watershed, Colorado, 1994-2007. 


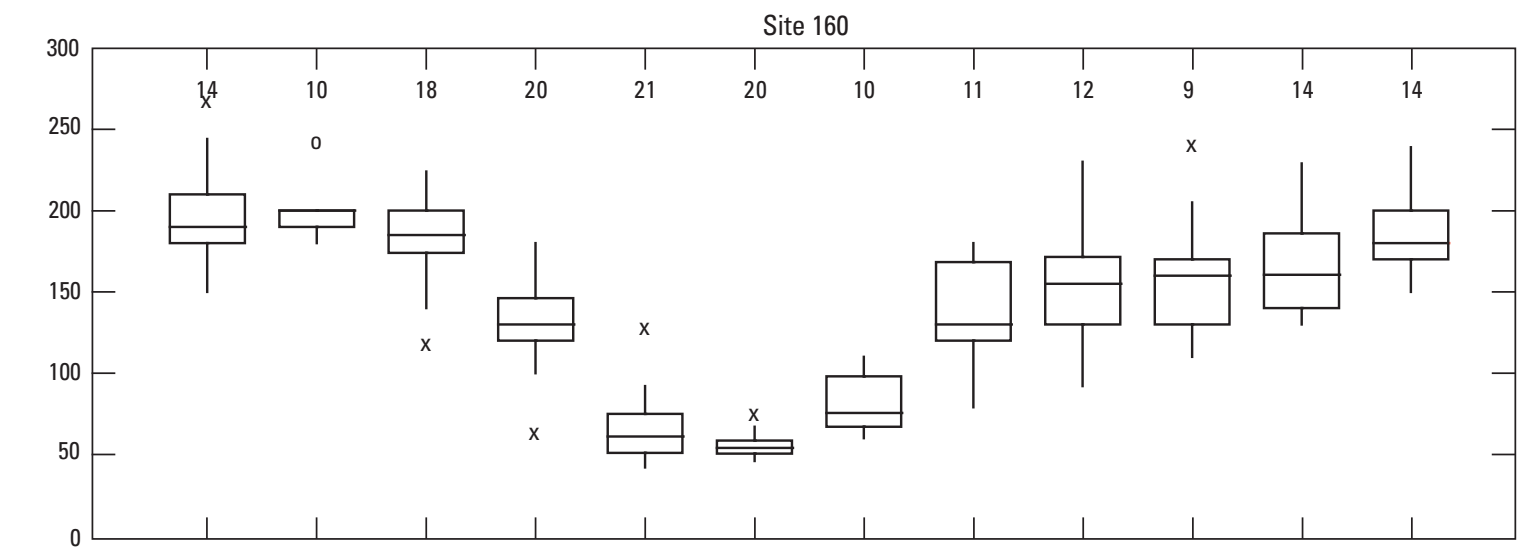

EXPLANATION

30 Number of values

o Upper detached

$\mathrm{x}$ Upper outside Upper adjacent

90th percentile

75th percentile

median

25th percentile

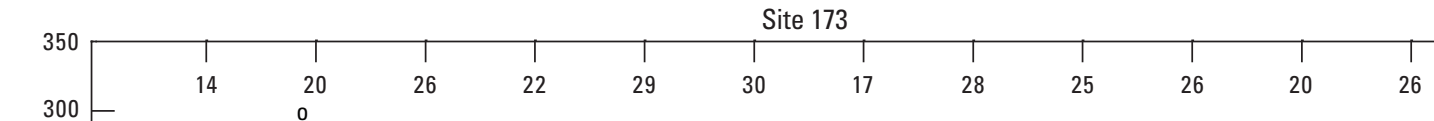

10th percentile Lower adjacent

Lower outside

- Lower detached
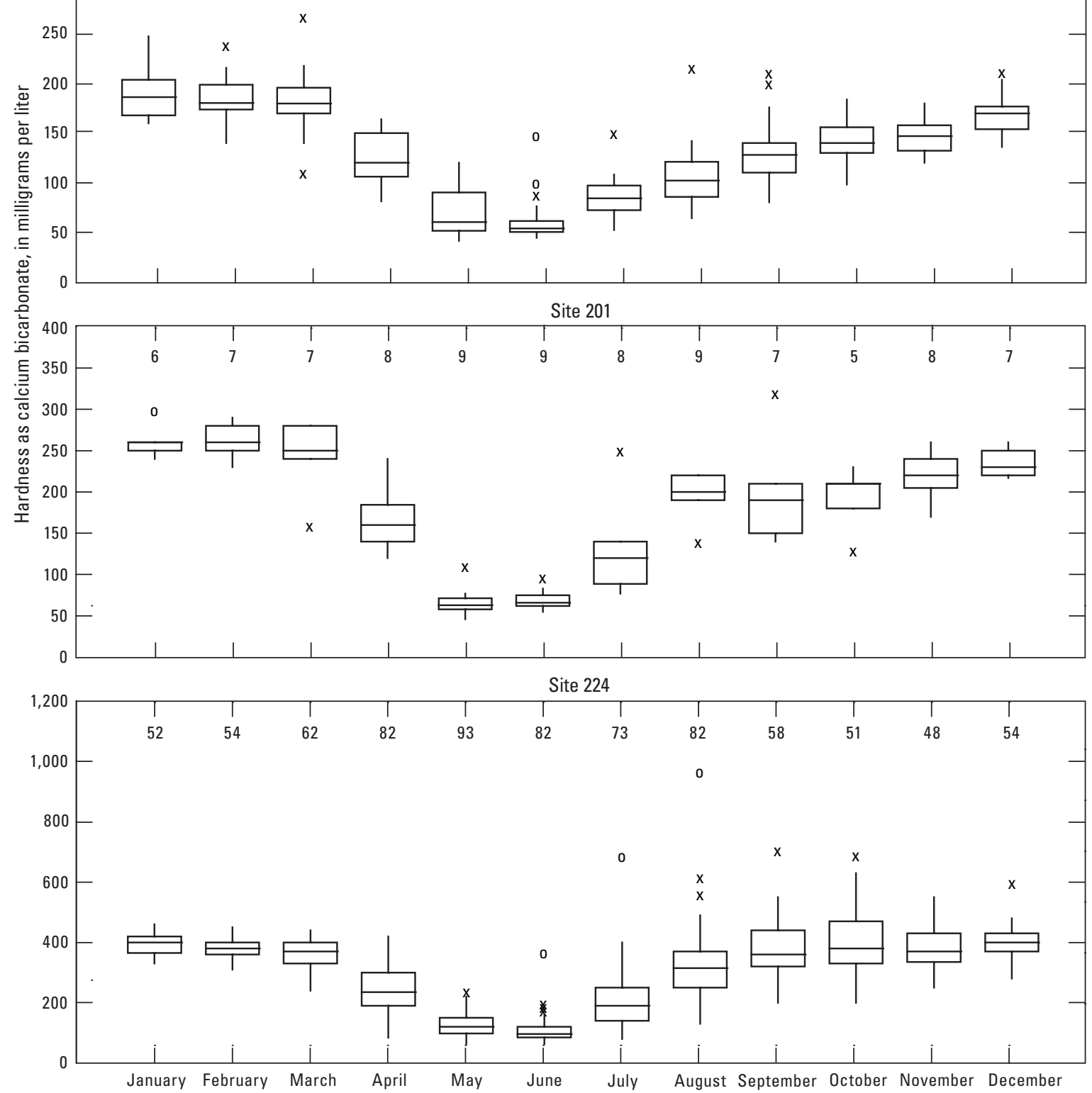

Figure 28. Seasonal pattern of hardness concentrations at selected sites in the Eagle River watershed, Colorado, 1947-2007. 
Table 11. Summary statistics of dissolved solids and major ions data in the Eagle River watershed, Colorado by subwatershed 1947-2007.

[No., number; MRL, Minimum Reporting Limit; --, undetermined; ROE, residue on evaporation; SOC, sum of constituents]

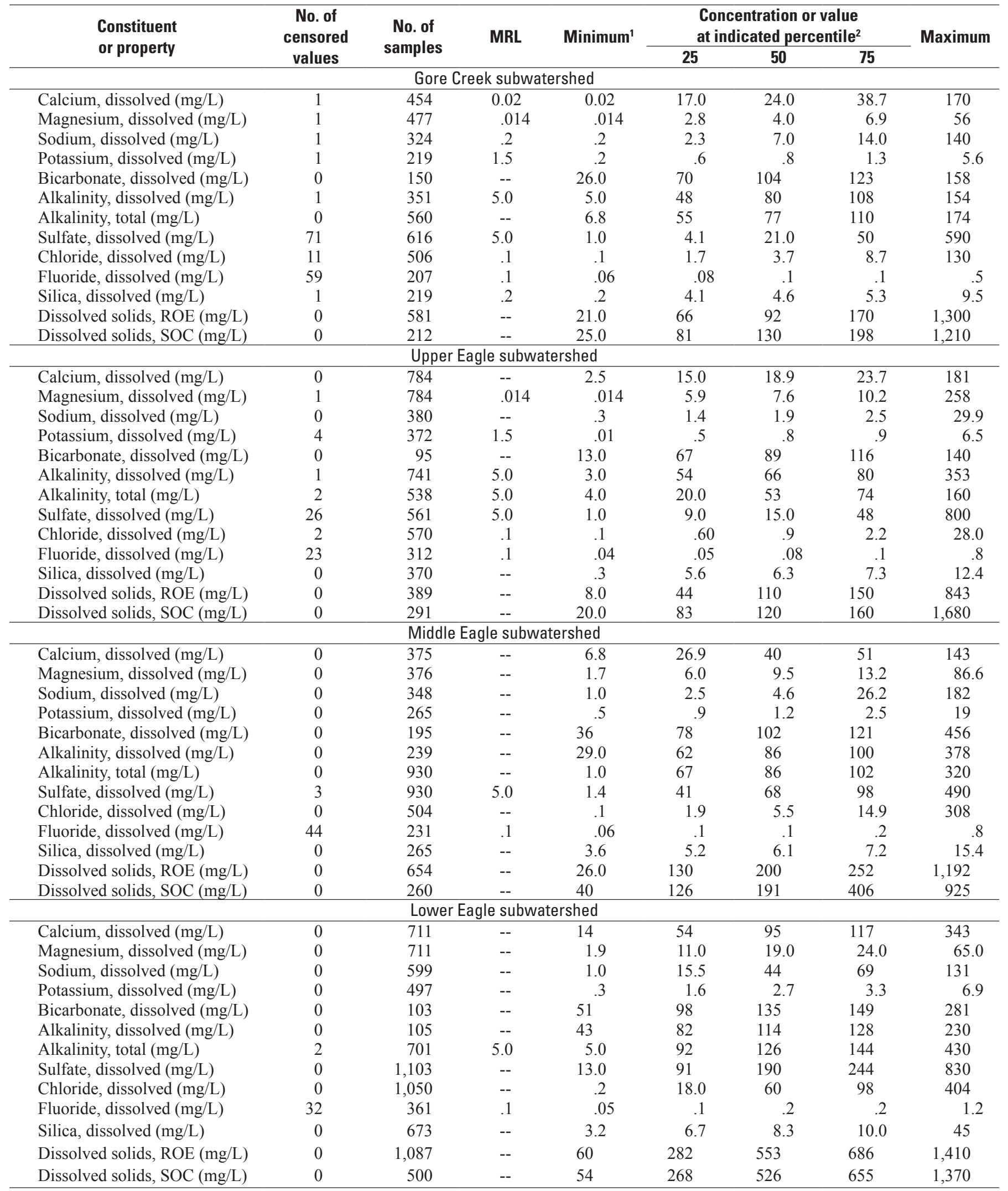

${ }^{1}$ Uncensored values.

${ }^{2}$ Percentile values can be less than the MRL values due to multiple MRLs within the combined dataset. 


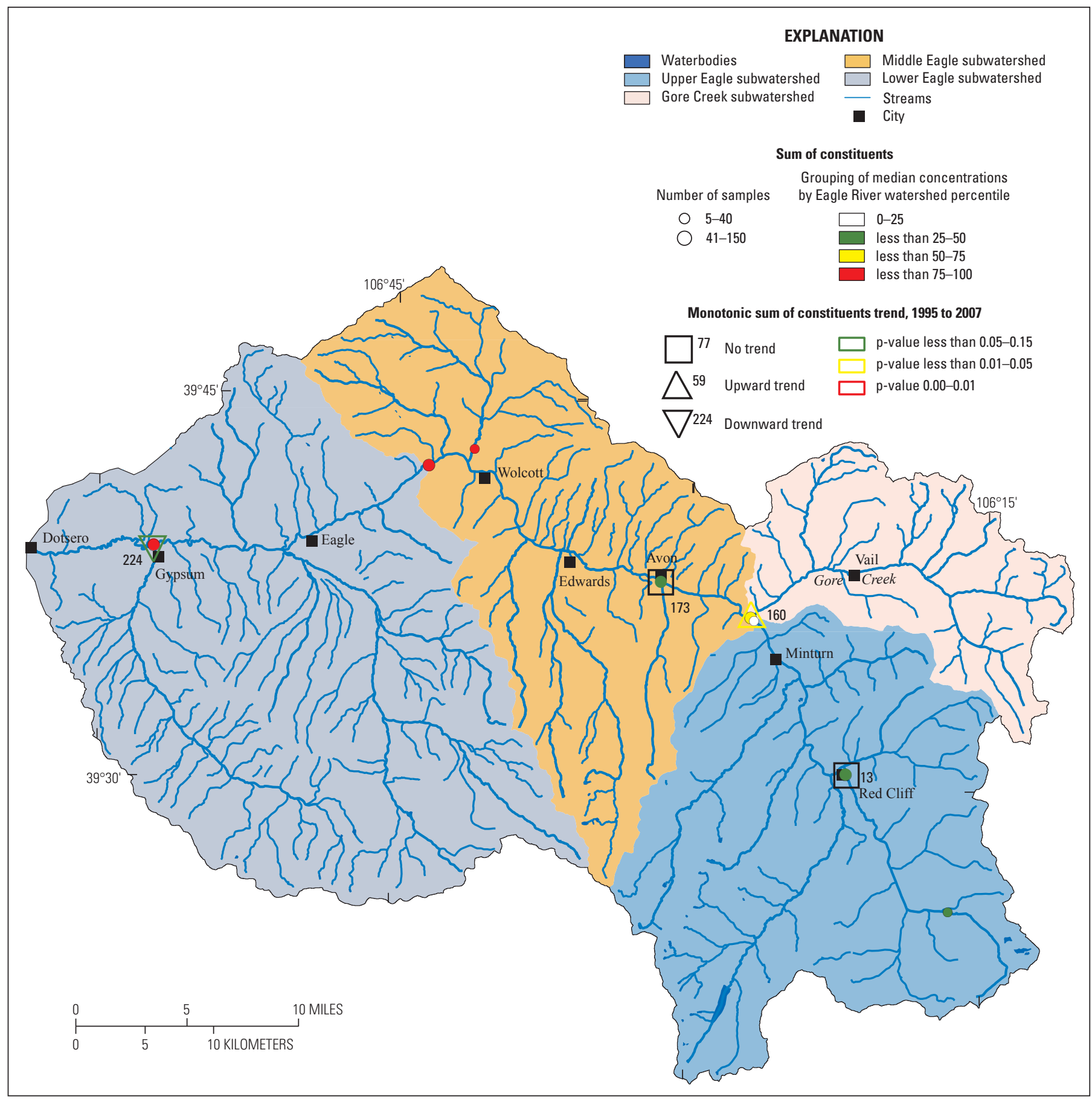

Base from U.S. Geological Survey digital data, 2007,

Universal Transverse Mercator projection

Zone 13

Figure 29. Spatial distribution of median-concentration percentiles and monotonic sum of constituent trends at selected sites in the Eagle River watershed, Colorado, 1995-2007. 


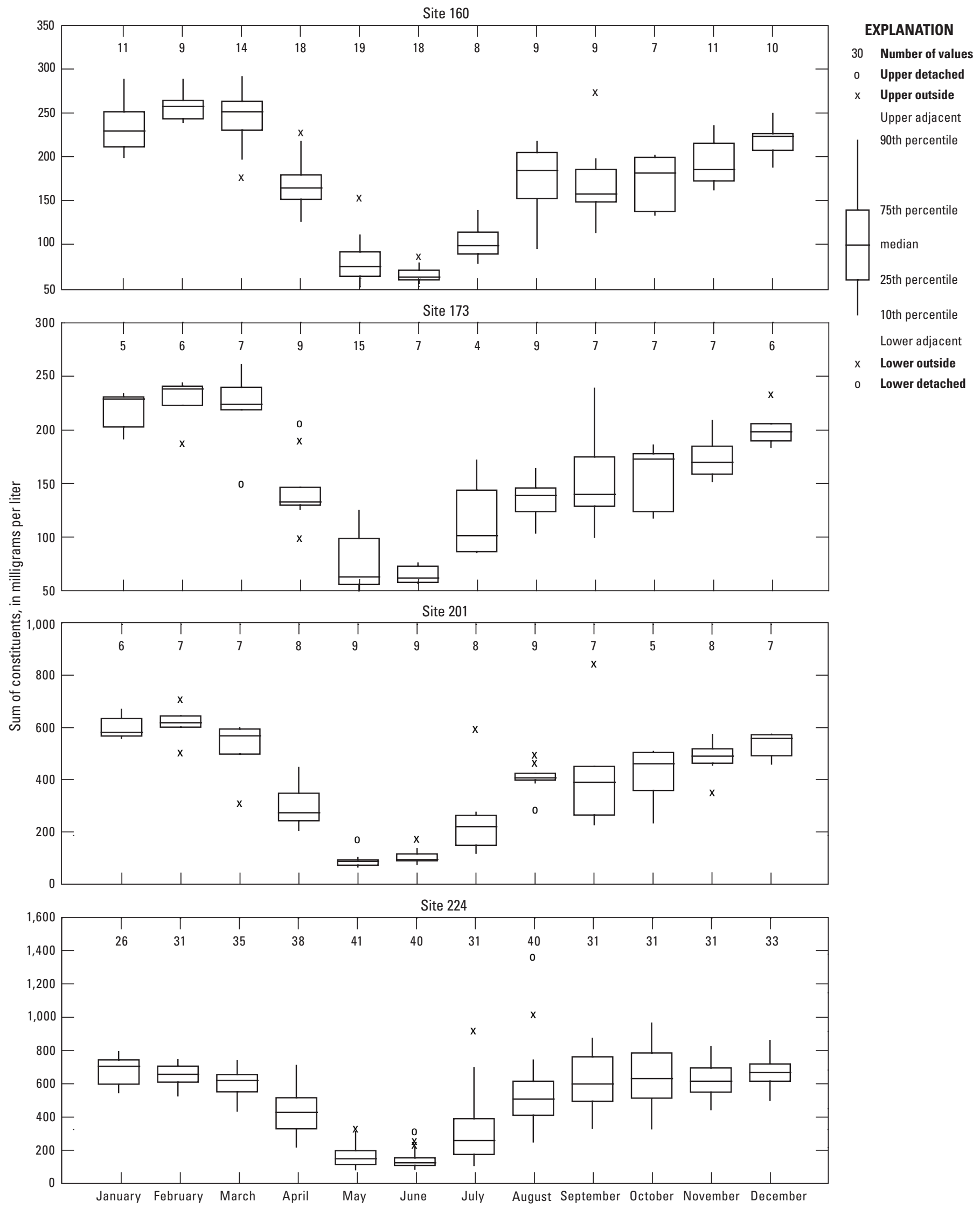

Figure 30. Seasonal pattern of sum of constituents at selected sites in the Eagle River watershed, Colorado, $1947-2007$. 
Table 12. Summary of monotonic temporal trends for dissolved solids and major ions in the Eagle River watershed, Colorado.

[No., number; --, undetermined, mg/L, milligrams per liter]

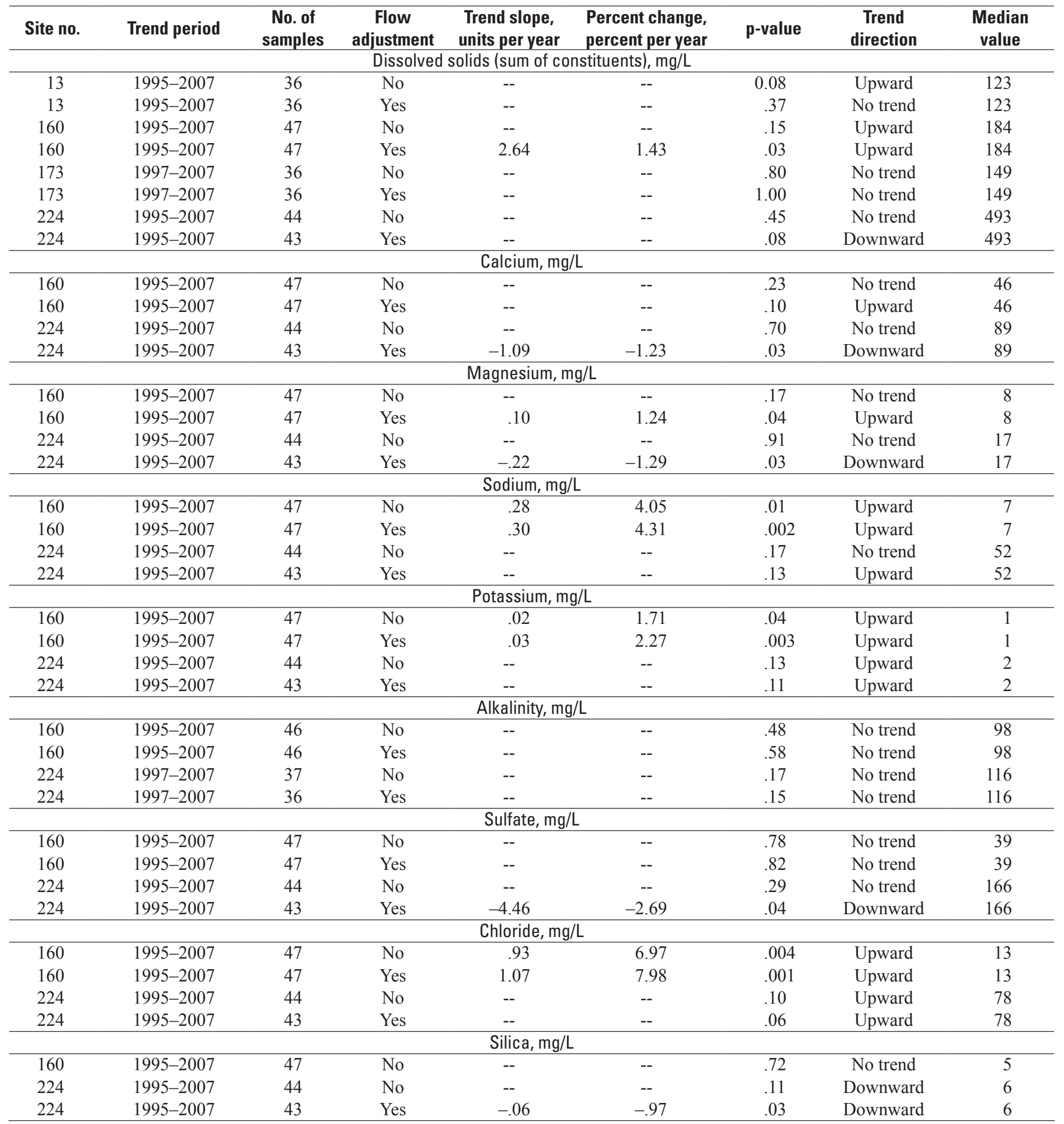


(p-value of 0.08) at site 224 (Lower Eagle subwatershed) shows decreasing TDS concentrations upstream from Gypsum. No flow-adjusted trend was detected on the Eagle River upstream from Avon (site 173) (Middle Eagle subwatershed) or upstream of Red Cliff (site 13) (Upper Eagle subwatershed). Areas between Gypsum and Avon may be contributing to the detected trend on the Eagle River at Gypsum.

A comparison of available data for major ions was done to determine ion-specific trends in the ERW. Spatial patterns were consistent with the patterns observed for the SOC method (fig. 29). Identification of the specific cations (positively charged ions) and anions (negatively charged ions) within a watershed can help identify likely source areas and also can aide in the evaluation of the causes of detected trends.

Trilinear diagrams or "Piper plots" are useful representations of the relative percentages of major ions within a waterquality sample and allow for the characterization of water types for specific sites and seasons. Trilinear diagrams are presented for selected sites from different areas of the ERW in figures 31-36. Separation of the data into three month time periods based on collection date (October-December, JanuaryMarch, April-June, and July-September) allows for an examination of seasonal variations in water chemistry at each site.

At sites 13 (Upper Eagle subwatershed) and 173 (Middle Eagle subwatershed), relative percentages of calcium, magnesium, and sodium plus potassium are consistent throughout the year, with changes occurring in the relative percentages of sulfate and bicarbonate with higher concentrations of bicarbonate potentially related to changes in water chemistry from snowmelt runoff April-June. Similar patterns are present in the relative abundance of calcium, magnesium, and sodium plus potassium and in the occurrence of higher concentrations of bicarbonate April-June in data from Gore Creek (site 160; Gore Creek subwatershed); however, variations exist in the concentrations of chloride plus fluoride with the higher concentrations occurring for some samples January-June. At sites 201 (Middle Eagle subwatershed) and 224 (Lower Eagle subwatershed), relative percentages of several ions vary by season. For the most part, the April-June time period shows relative increases in calcium and bicarbonate and decreases in chloride and sulfate (figs. 34 and 35).

In addition to temporal variations, regional variations in the relative percent of major ions also appear to exist. Sites in areas downstream from the Eagle Valley Evaporite (site 224) appear to have higher relative percentages of sodium plus potassium and chloride plus fluoride than sites upstream from the Eagle Valley Evaporite. These differences appear to be consistent with the geology of the two areas, with an exception during the January-June time period in Gore Creek where increases in chloride do not appear to strongly correlate to geologic sources.

The spatial patterns of each major ion were similar to the patterns observed in the SOC method and ROE method data (figs. 37-44). Localized areas with higher concentrations of calcium were observed on Rock Creek and Alkali Creek (fig. 37). Localized areas with higher concentrations of magnesium were observed on the East Fork of the Eagle River near Red Cliff, Rock Creek, and Alkali Creek (fig. 38). Localized areas with higher concentrations of sodium were observed on Black Gore Creek and Alkali Creek (fig. 39). These localized areas may be related to human or geologic effects and there is insufficient data to determine an exact source. Localized areas with higher concentrations of alkalinity (fig. 44) were observed on the East Fork of the Eagle River near Red Cliff, Black Gore Creek, and Alkali Creek.

Chloride concentrations measured in samples were compared to the State standards within the ERW to provide an environmentally relevant context to observations of stream condition. The State standard for chloride $(250 \mathrm{mg} / \mathrm{L})$ (Colorado Department of Public Health and Environment, 2008) was exceeded less than 1 percent ( 3 comparisons) out of the 2,630 comparisons in the ERW (table 13 and fig. 45).

The two sites with detected trends in TDS (SOC method), site 160 (Gore Creek subwatershed upward trend) and site 224 (Lower Eagle subwatershed downward trend), were evaluated for trends in calcium, magnesium, sodium, potassium, alkalinity, sulfate, chloride, and silica for the time period 1995-2007 (table 12, figs. 37-44). At site 160 (Gore Creek subwatershed), highly significant flow-adjusted upward trends were found for sodium ( $\mathrm{p}$-value of 0.002), potassium (p-value of 0.003), and chloride (p-value of 0.001); with a significant upward trend in magnesium (p-value of 0.04); and a weakly significant trend in calcium ( $p$-value of 0.10 ). At site 160 (Gore Creek subwatershed), no trends were observed in alkalinity, sulfate, or silica. At site 224 (Lower Eagle subwatershed), significant flow-adjusted downward trends were detected in calcium ( $\mathrm{p}$-value of 0.03 ), magnesium ( $\mathrm{p}$-value of 0.03), sulfate (p-value of 0.04), and silica (p-value of 0.03 ); and weakly significant upward trends were observed in sodium (p-value of 0.13), potassium ( $\mathrm{p}$-value of 0.11 ), and chloride ( $\mathrm{p}$-value of 0.06). At site 224 (Lower Eagle subwatershed), no trend was detected in alkalinity.

A quantitative analysis of the relative abundance of calcium, magnesium, sodium, and potassium [cations with a significant upward trend at site 160 (Gore Creek subwatershed)] to the available anions (anions with a significant upward trend at site 160) indicates that chloride salts likely are the source for the detected upward trends because chloride is the only commonly occurring anion with a trend at site 160 . To deduce the source minerals contributing to the observed trends the rate-of-change (increase in ions in $\mathrm{mg} / \mathrm{L}$ per year) for each cation and anion was converted to milliequivalents. For example, if chloride is the anion related to increases in sodium there needs to be an equal increase in chloride to balance the atomic ratio of sodium chloride $(\mathrm{NaCl})$; the same one-to-one ratio is needed for potassium chloride $(\mathrm{KCl})$. Likewise, for chloride to be the anion related to the observed increases in magnesium there needs to be twice the increase in chloride to balance the atomic ratio of magnesium chloride $\left(\mathrm{MgCl}_{2}\right)$; the same one-to-two ratio is needed for calcium chloride $\left(\mathrm{CaCl}_{2}\right)$. To show strong evidence that chloride is the anion related to the cation trends at site 160, the number of milliequivalents of 

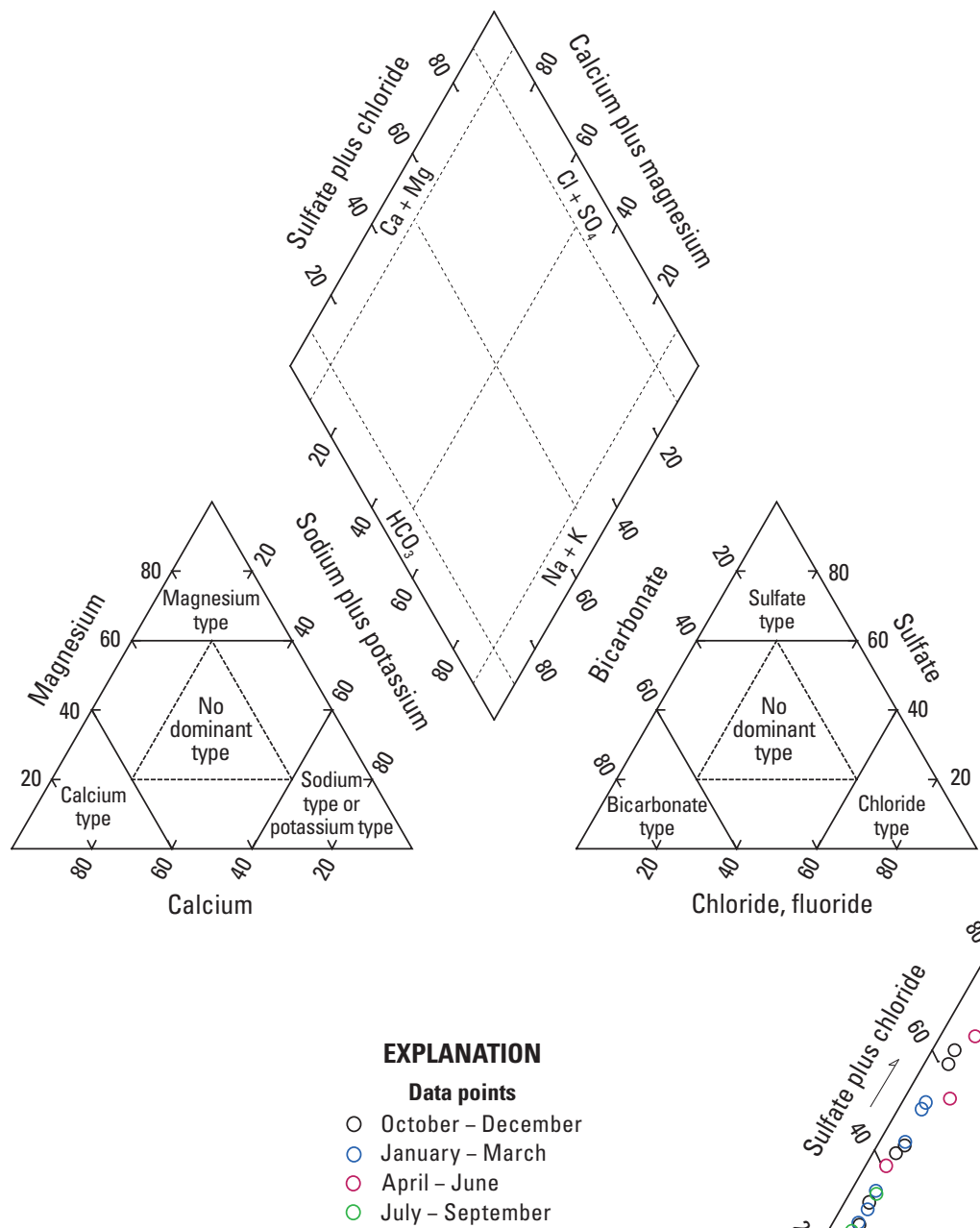

tember

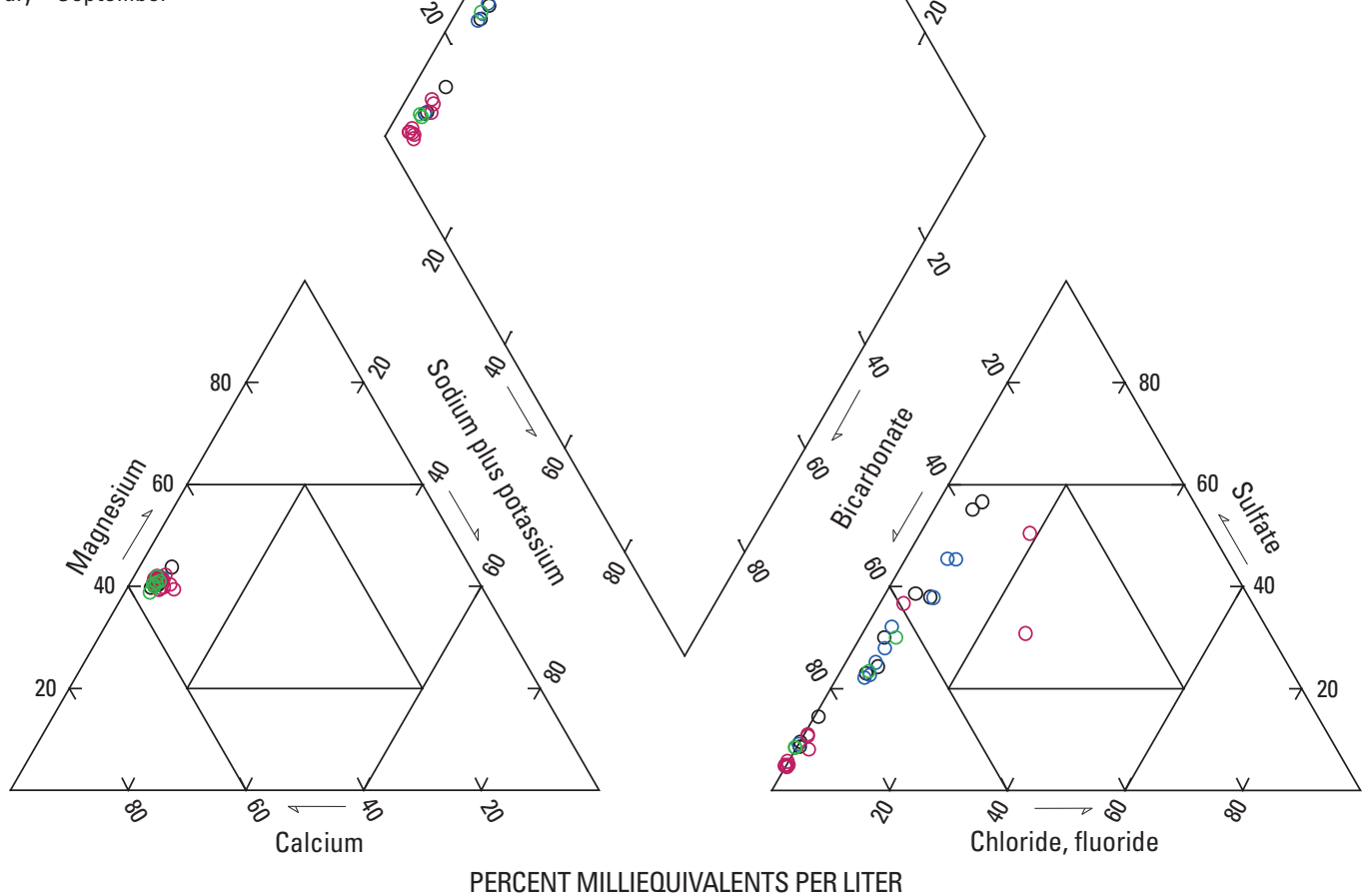

Figure 31. Seasonality of water chemistry at site 13, Eagle River watershed, Colorado, 1947-2007. 

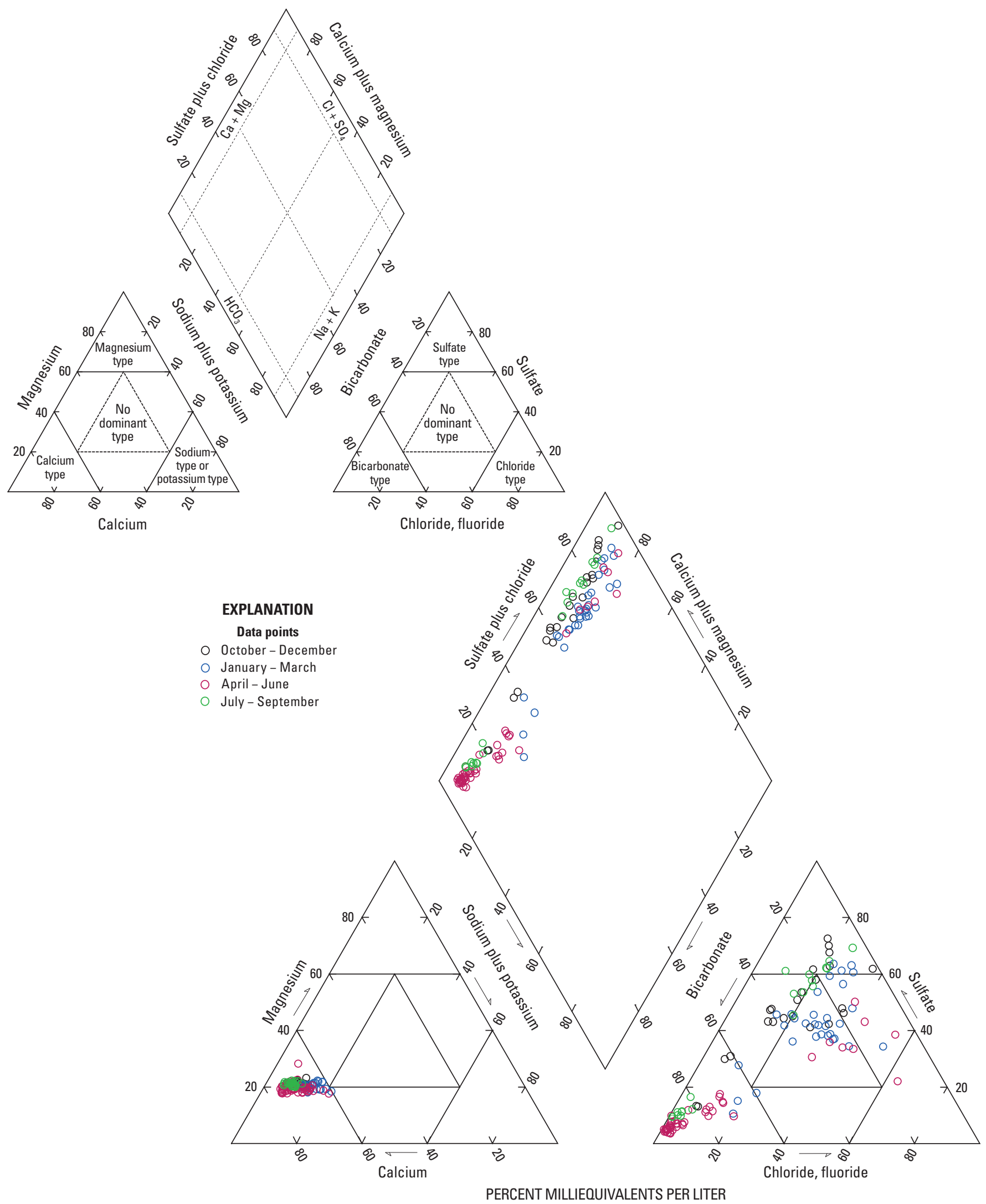

Figure 32. Seasonality of water chemistry at site 160, Eagle River watershed, Colorado, 1947-2007. 

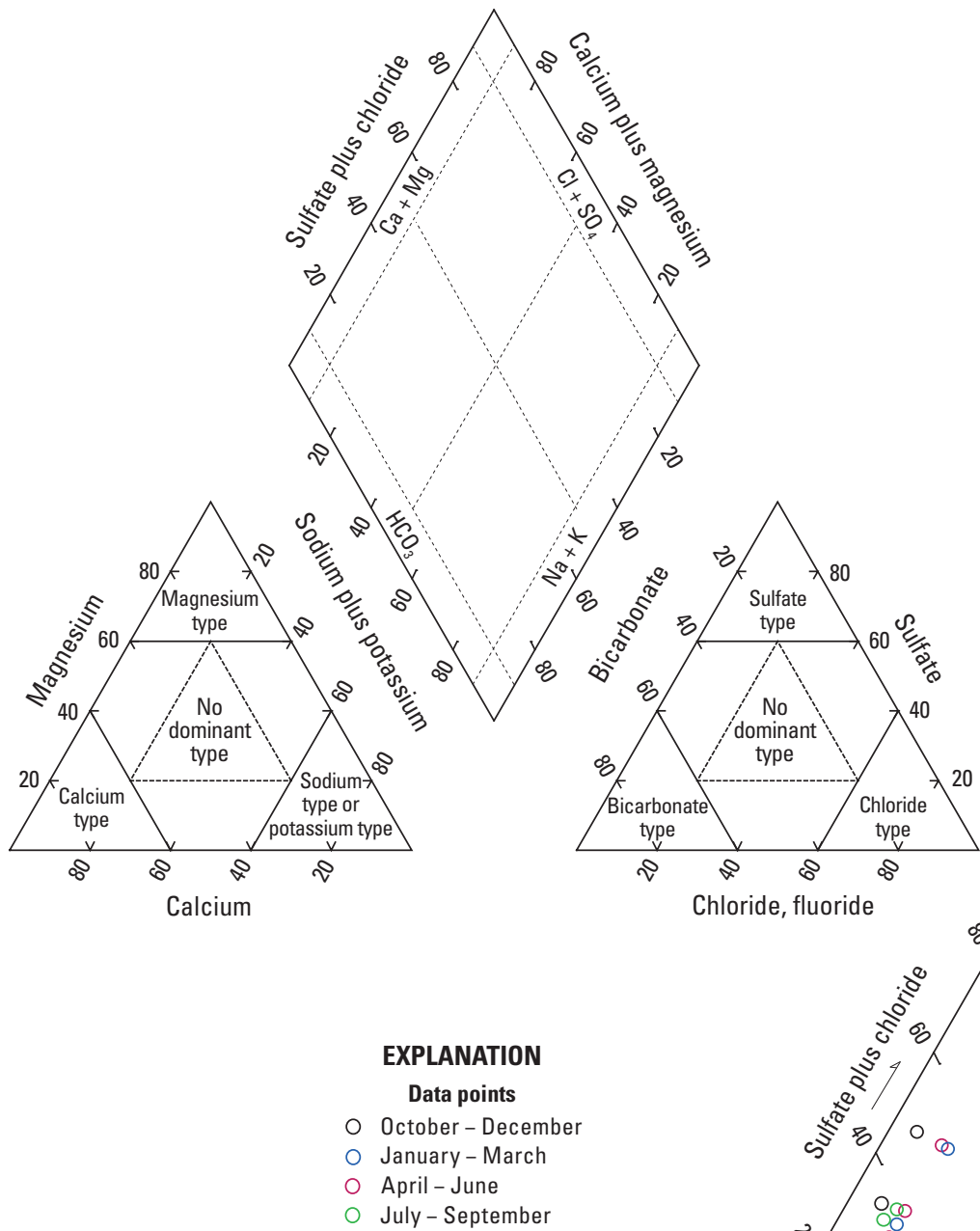

July - Septemb

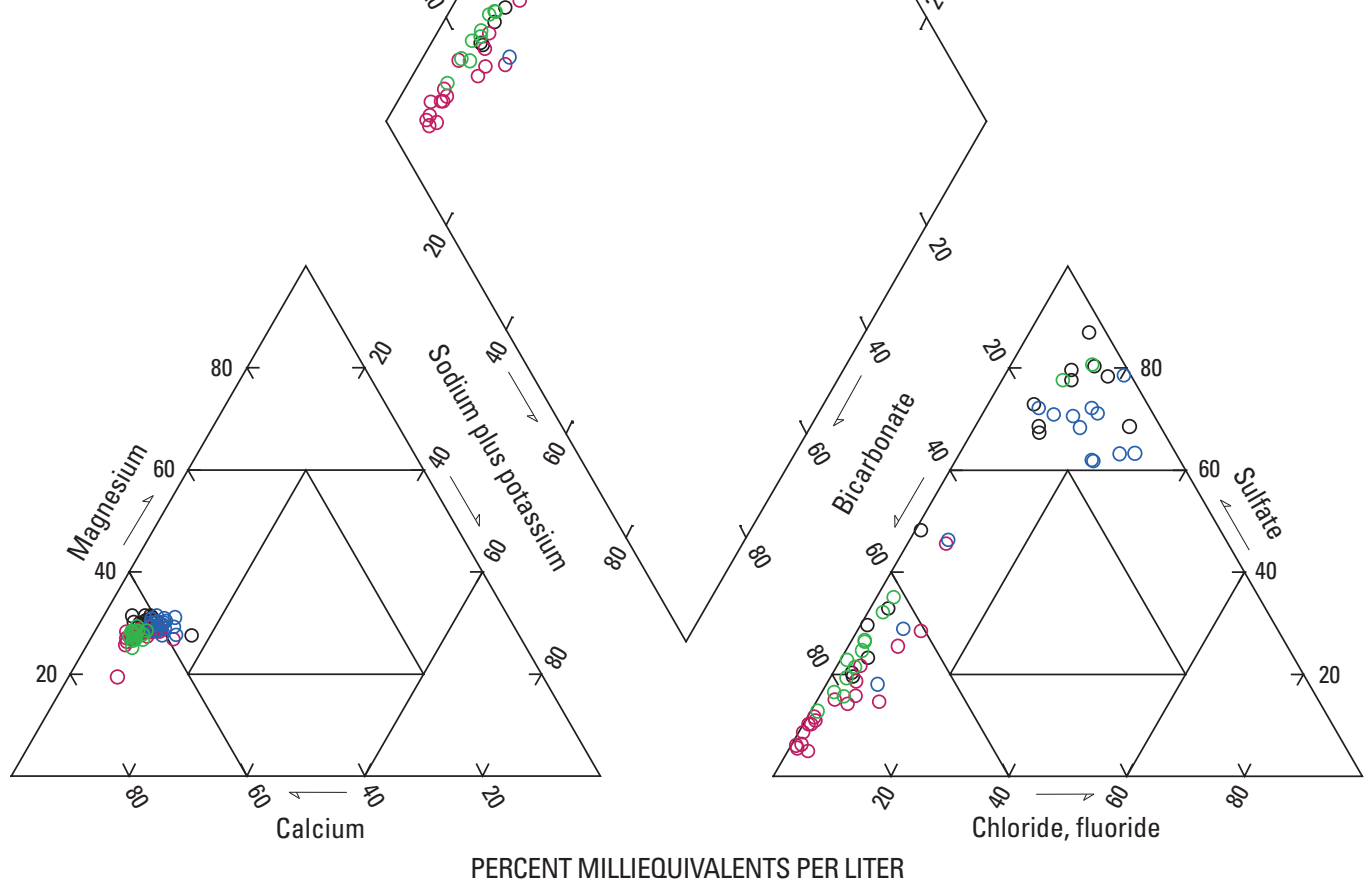

Figure 33. Seasonality of water chemistry at site 173, Eagle River watershed, Colorado, 1947-2007. 

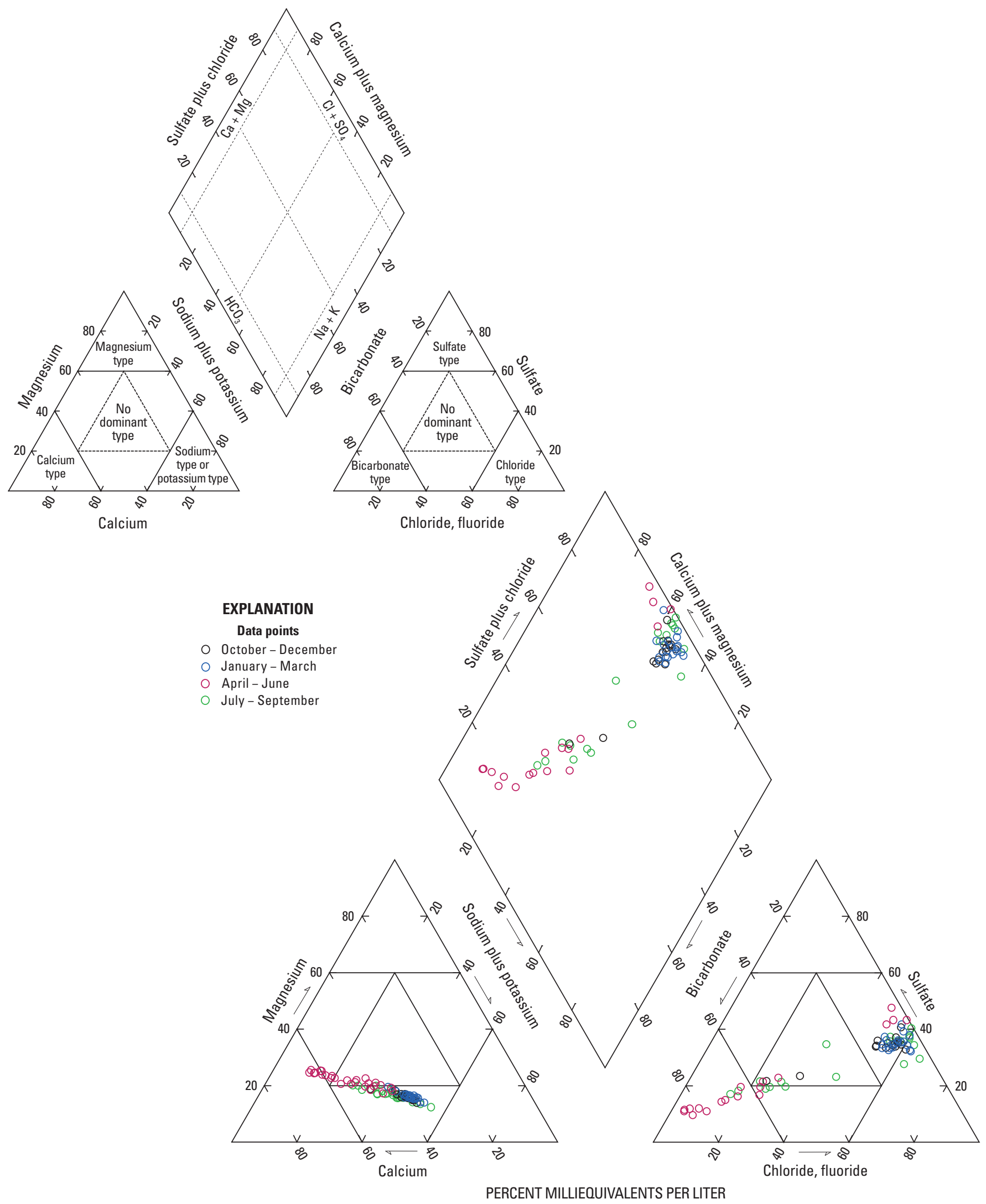

Figure 34. Seasonality of water chemistry at site 201, Eagle River watershed, Colorado, 1947-2007. 

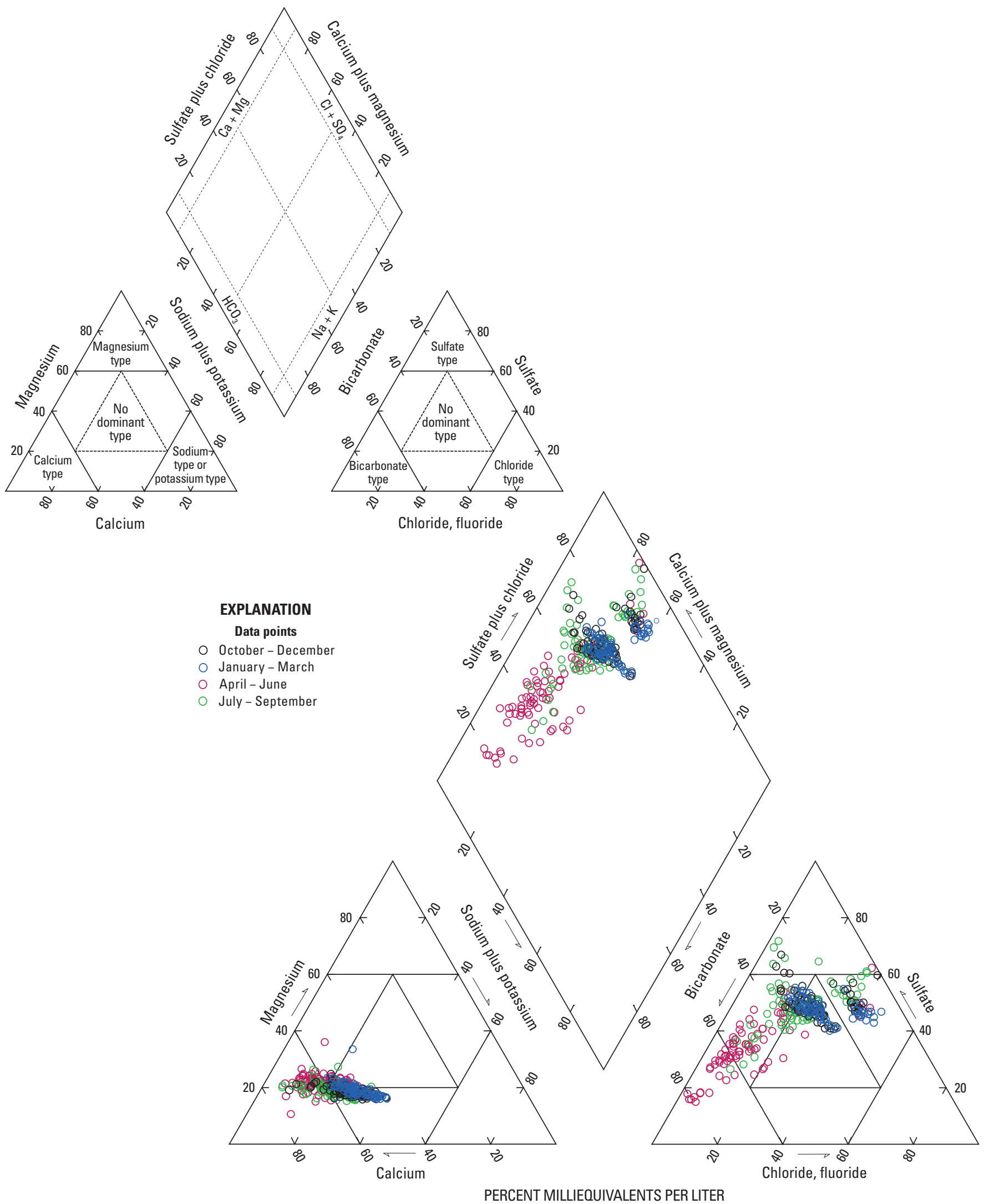

Figure 35. Seasonality of water chemistry at site 224, Eagle River watershed, Colorado, 1947-2007. 

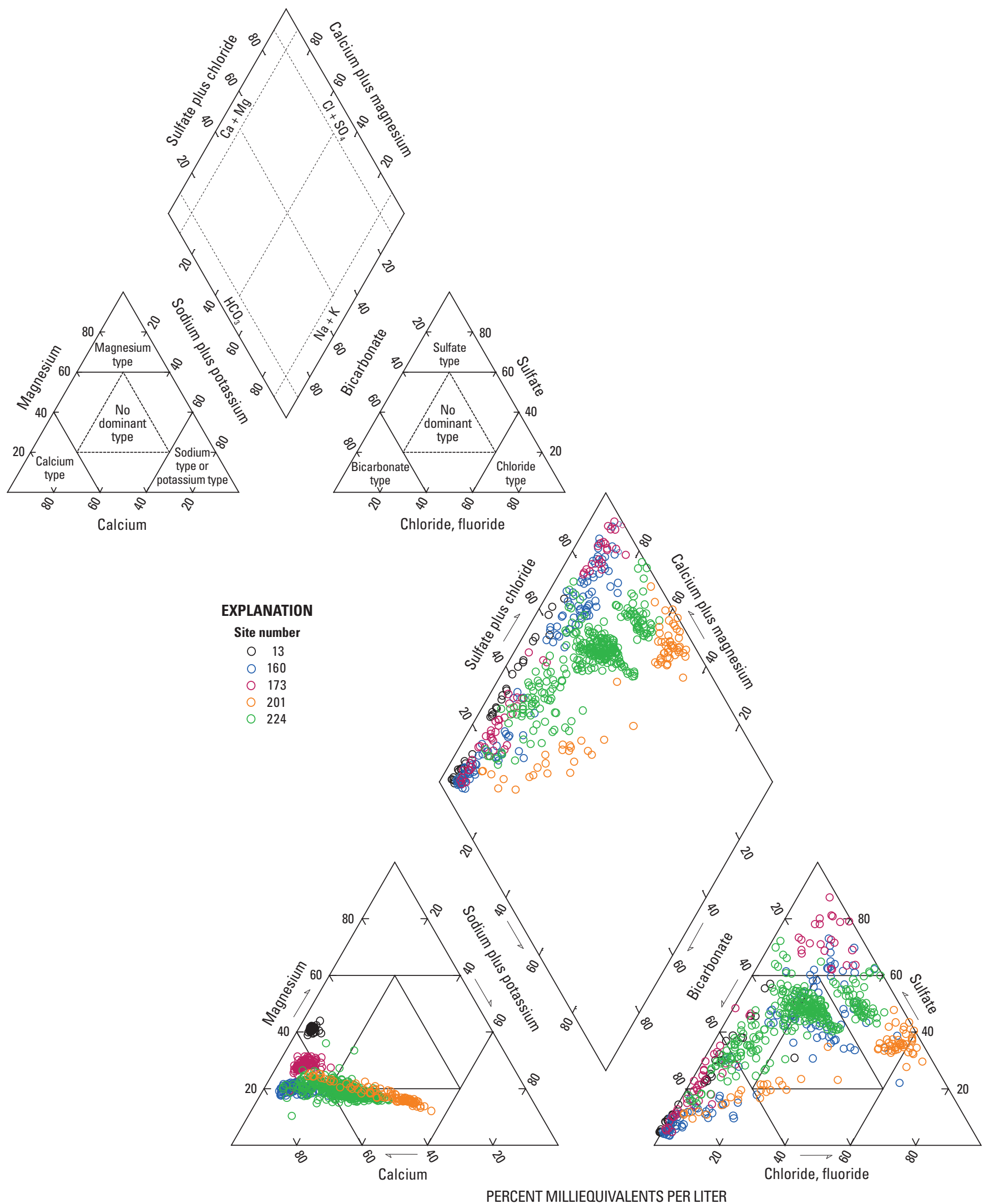

Figure 36. Seasonality of water chemistry at selected streamflow-gaging station locations, in the Eagle River watershed, Colorado, 1947-2007. 


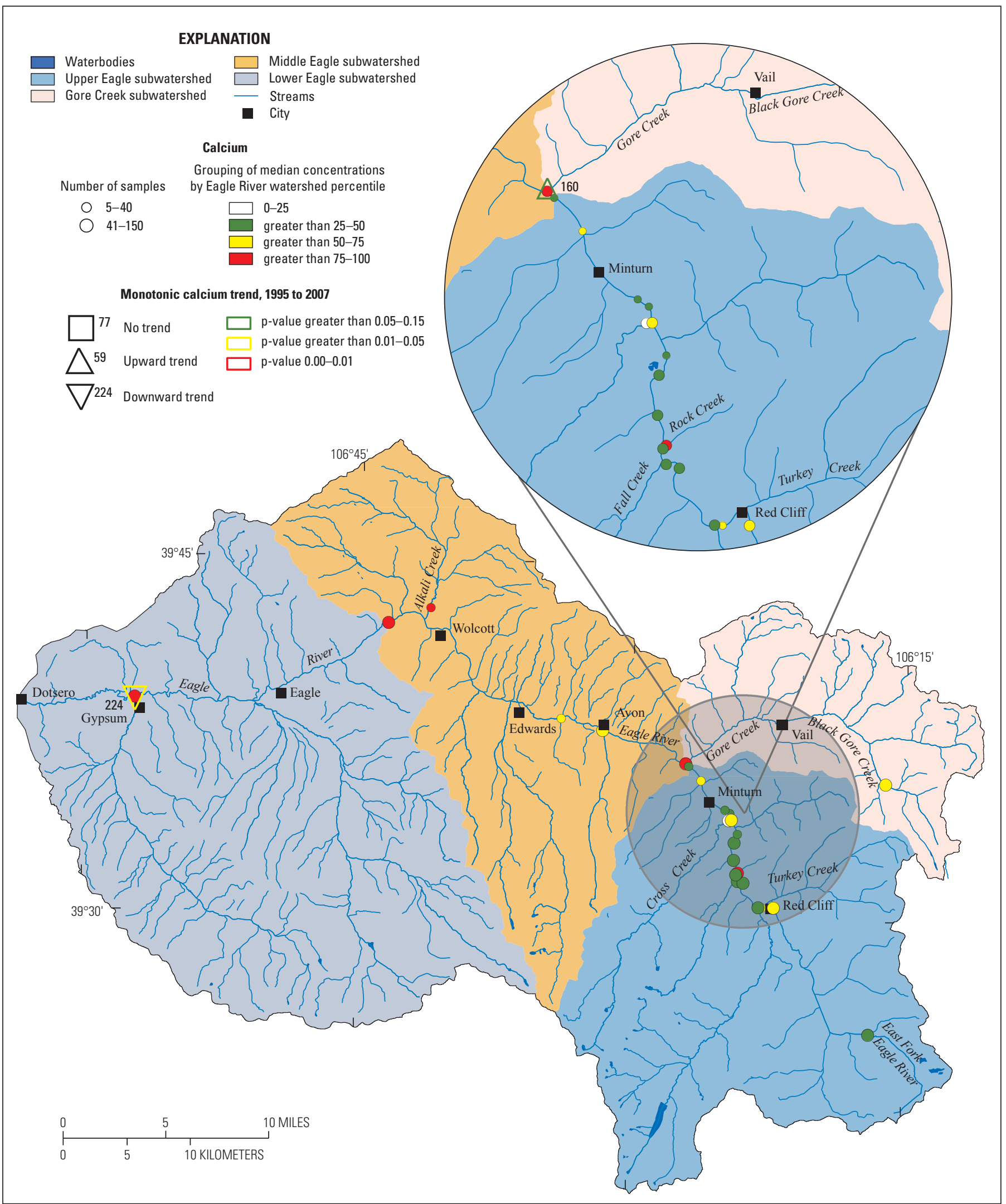

Base from U.S. Geological Survey digital data, 2007,

Universal Transverse Mercator projection

Zone 13

Figure 37. Spatial distribution of median-concentration percentiles and monotonic calcium concentration trends at selected sites in the Eagle River watershed, Colorado, 1995-2007. 


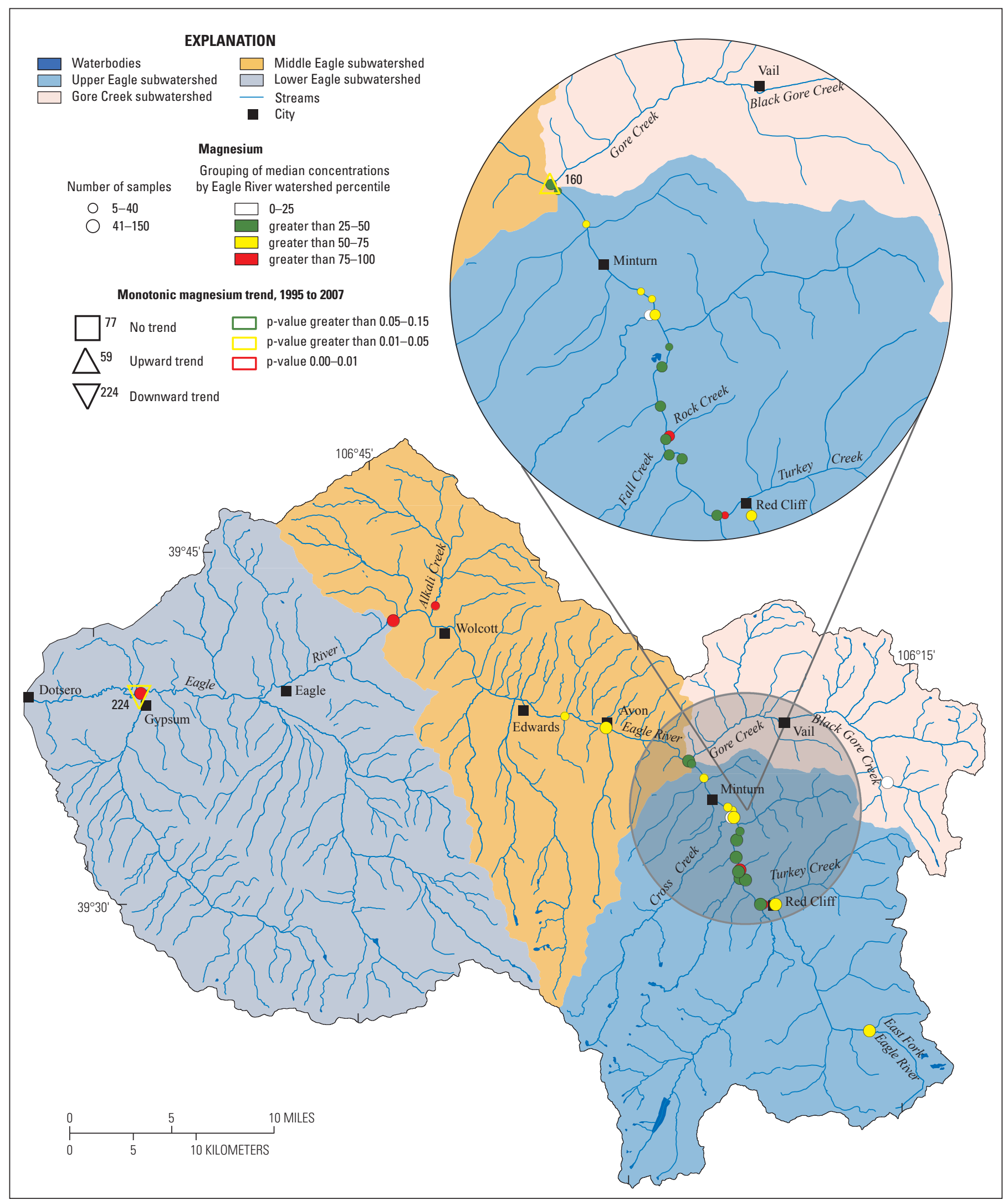

Base from U.S. Geological Survey digital data, 2007,

Universal Transverse Mercator projection

Zone 13

Figure 38. Spatial distribution of median-concentration percentiles and monotonic magnesium trends at selected sites in the Eagle River watershed, Colorado, 1995-2007. 


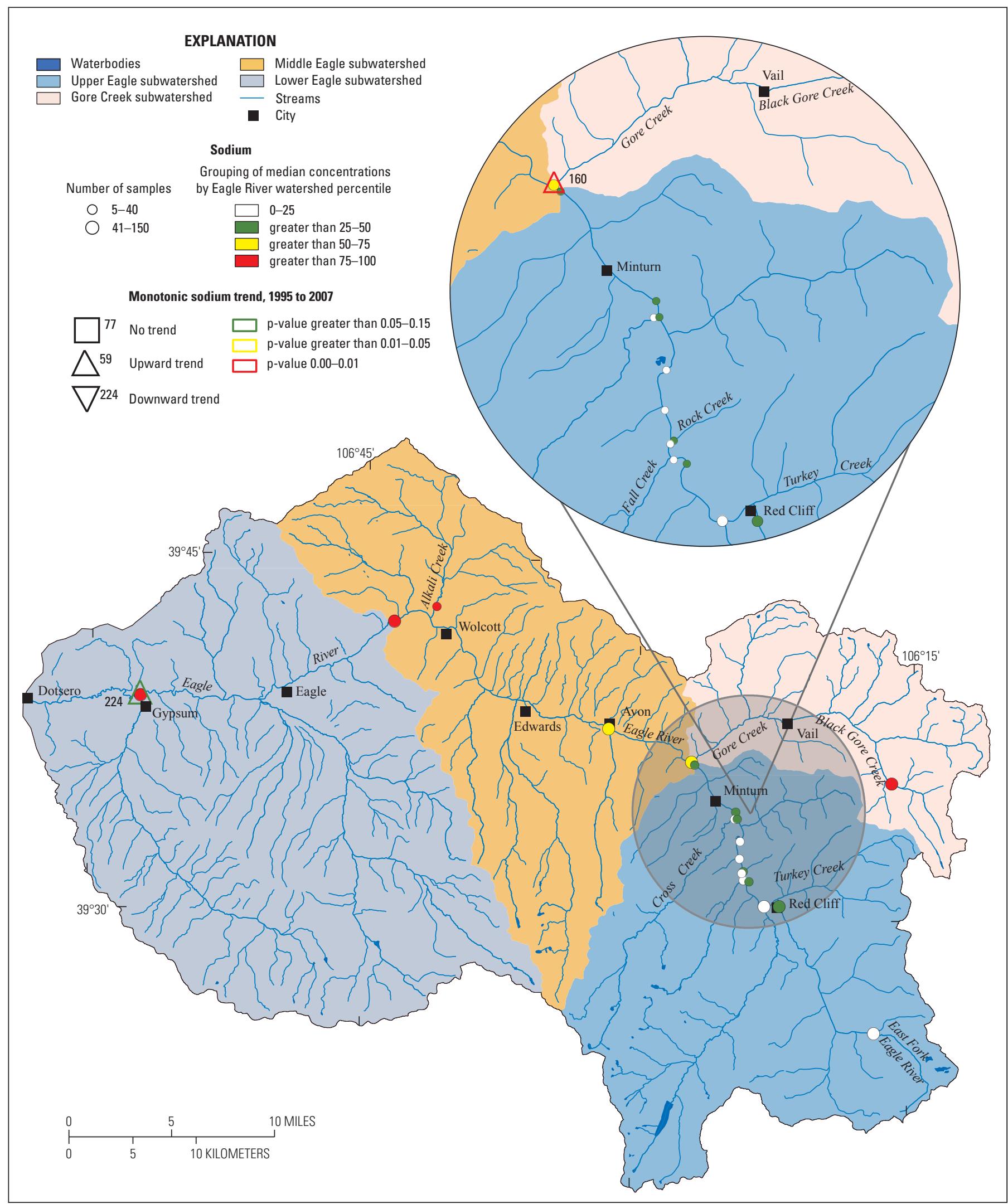

Base from U.S. Geological Survey digital data, 2007,

Universal Transverse Mercator projection

Zone 13

Figure 39. Spatial distribution of median-concentration percentiles and monotonic sodium trends at selected sites in the Eagle River watershed, Colorado, 1995-2007. 


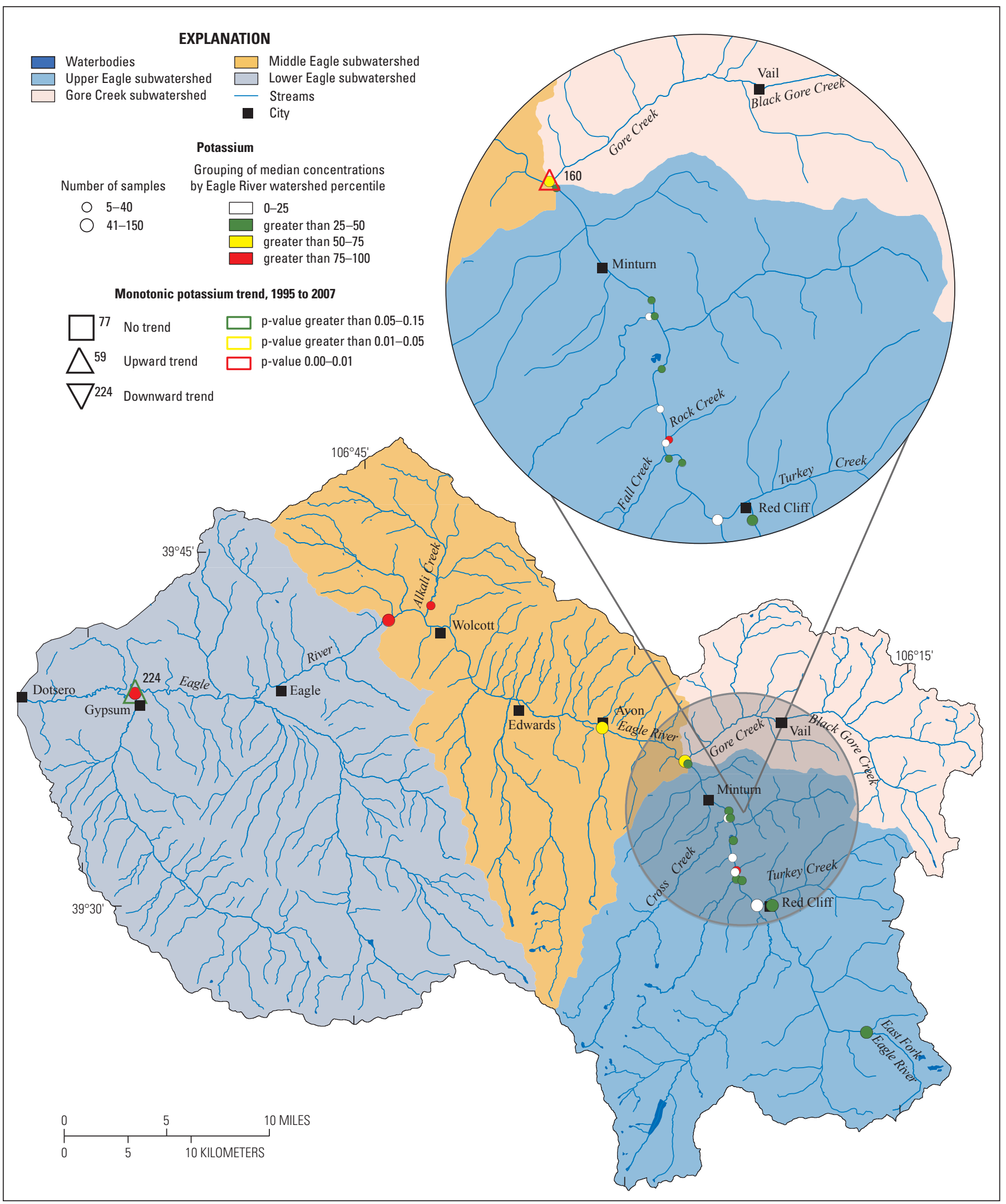

Base from U.S. Geological Survey digital data, 2007,

Universal Transverse Mercator projection

Zone 13

Figure 40. Spatial distribution of median-concentration percentiles and monotonic potassium trends at selected sites in the Eagle River watershed, Colorado, 1995-2007. 


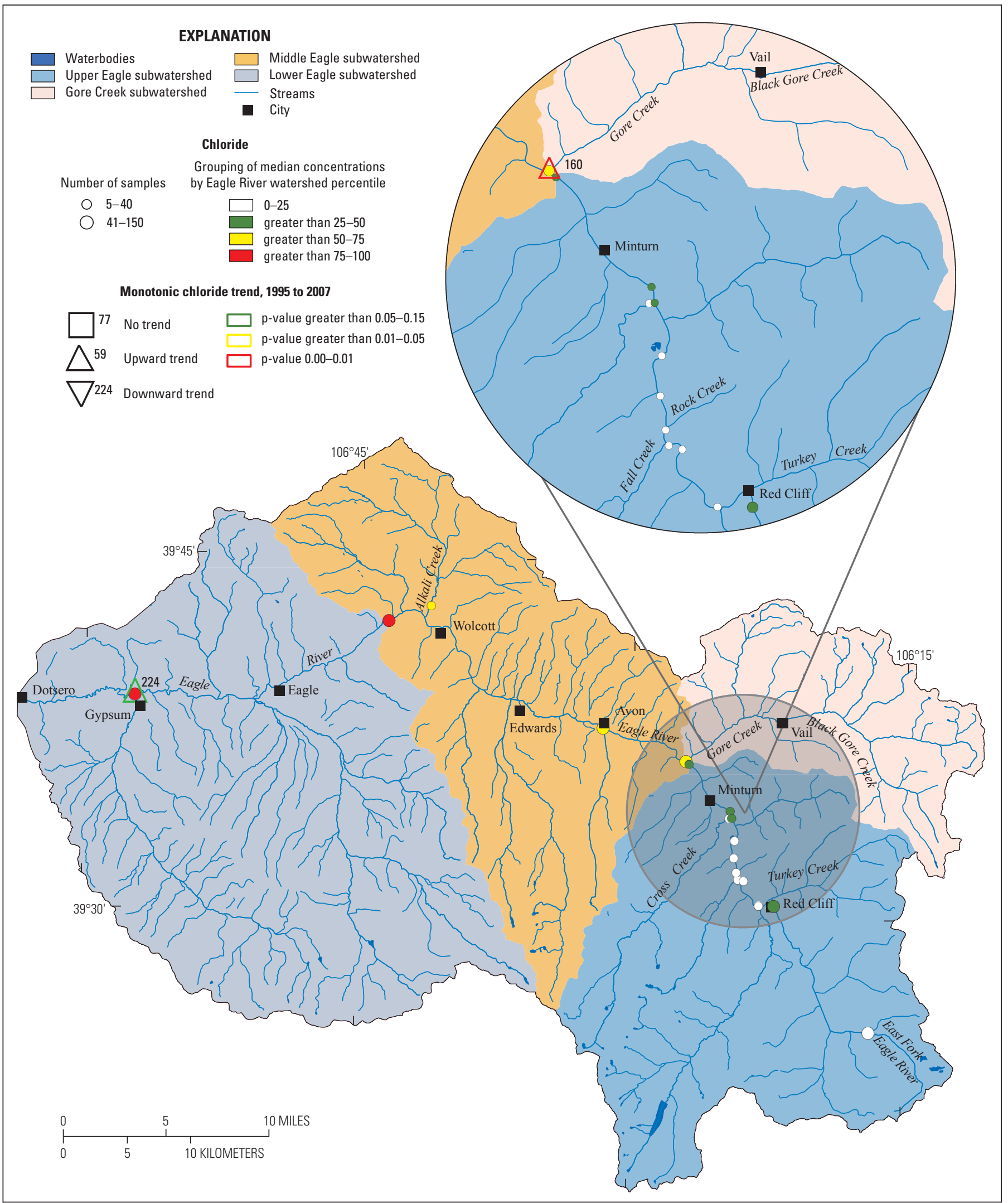

Base from U.S. Geological Survey digital data, 2007,

Universal Transverse Mercator projection

Zone 13

Figure 41. Spatial distribution of median-concentration percentiles and monotonic chloride trends at selected sites in the Eagle River watershed, Colorado, 1995-2007. 


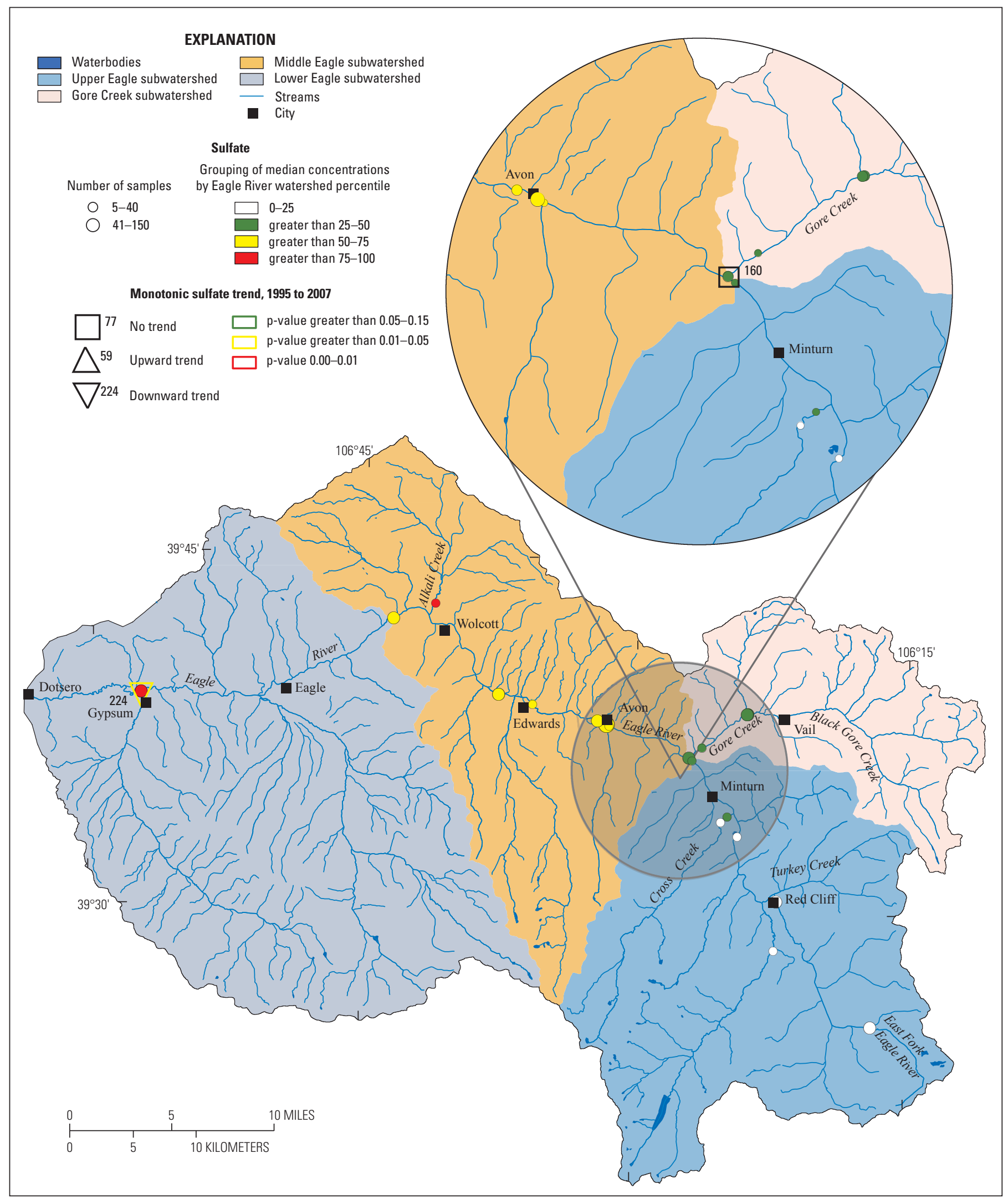

Base from U.S. Geological Survey digital data, 2007,

Universal Transverse Mercator projection

Zone 13

Figure 42. Spatial distribution of median-concentration percentiles and monotonic sulfate trends at selected sites in the Eagle River watershed, Colorado, 1995-2007. 


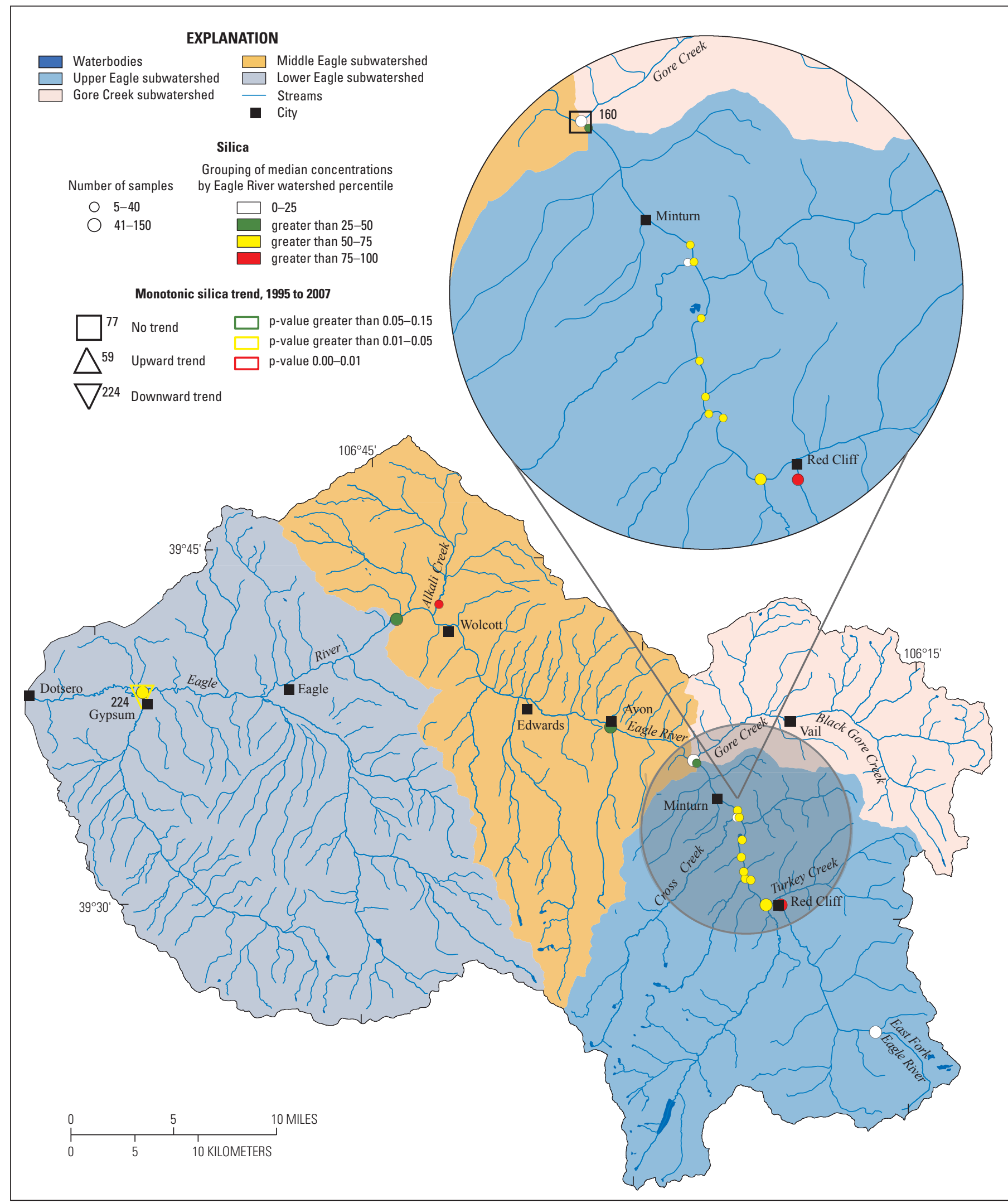

Base from U.S. Geological Survey digital data, 2007,

Universal Transverse Mercator projection

Zone 13

Figure 43. Spatial distribution of median-concentration percentiles and monotonic silica trends in at selected sites in the Eagle River watershed, Colorado, 1995-2007. 


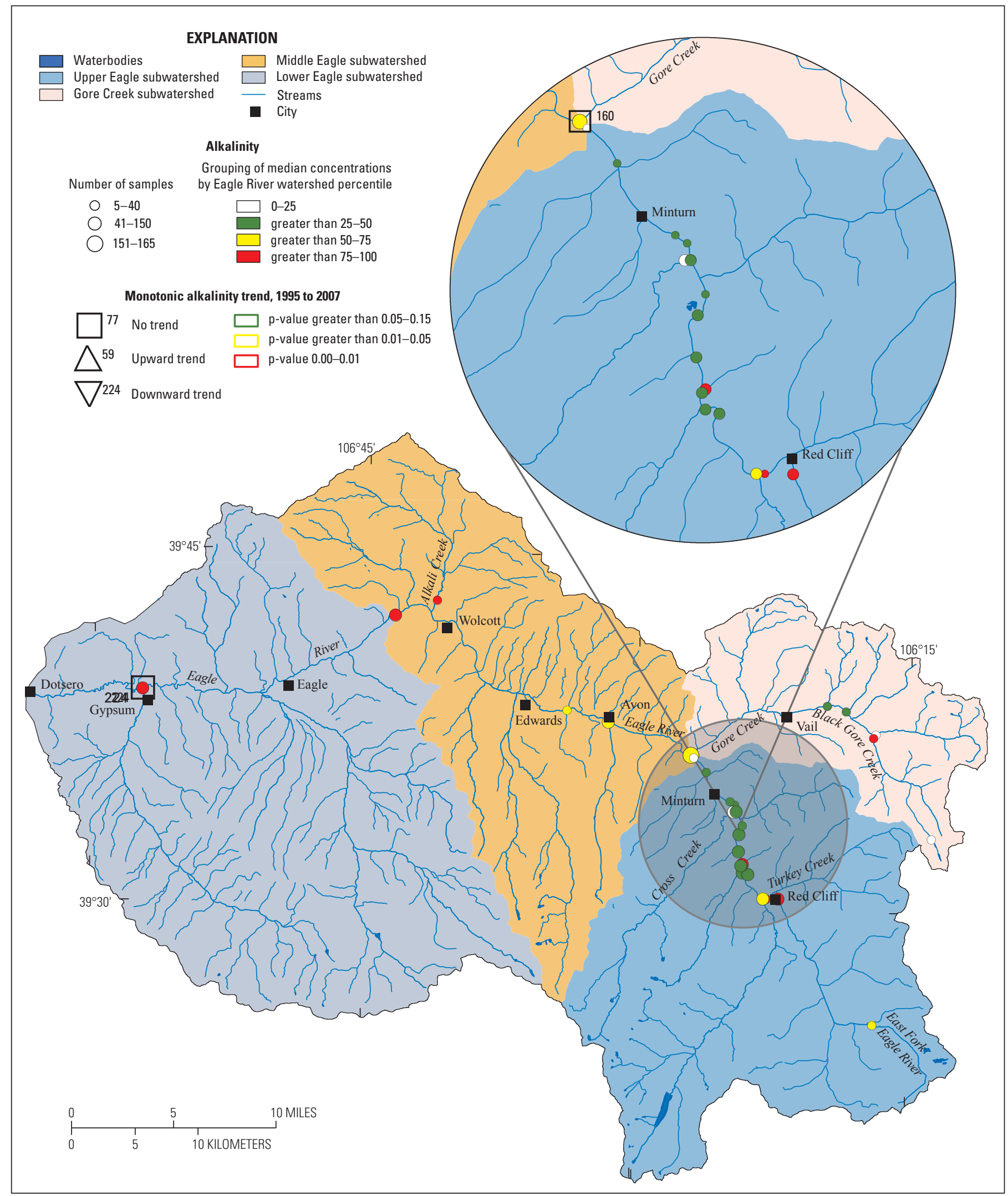

Base from U.S. Geological Survey digital data, 2007,

Universal Transverse Mercator projection

Zone 13

Figure 44. Spatial distribution of median-concentration percentiles and monotonic alkalinity trends at selected sites in the Eagle River watershed, Colorado, 1995-2007. 


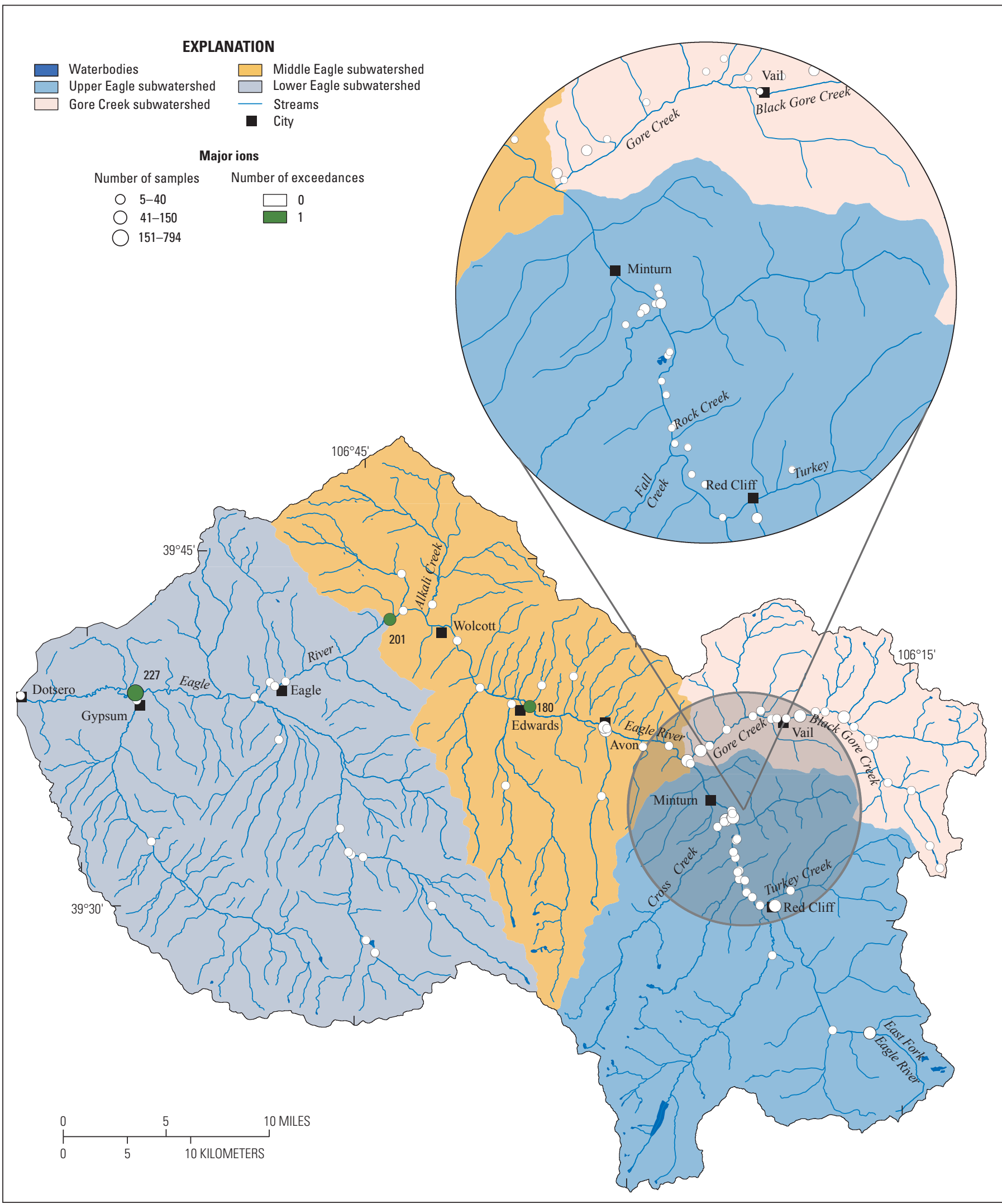

Base from U.S. Geological Survey digital data, 2007,

Universal Transverse Mercator projection

Zone 13

Figure 45. Number of exceedances of the Colorado water-quality standard for major ions at selected sites in the Eagle River watershed, Colorado, 1947-2007. 
Table 13. Summary of exceedances of Colorado water-quality standard (250 milligrams per liter) for chloride for surface-water samples collected in the Eagle River watershed, 1947-2007.

[No., number; --, no data]

\begin{tabular}{|c|c|c|c|c|c|}
\hline $\begin{array}{l}\text { Constituent } \\
\text { or property }\end{array}$ & $\begin{array}{c}\text { No. of } \\
\text { censored values }\end{array}$ & $\begin{array}{c}\text { No. of } \\
\text { samples }\end{array}$ & $\begin{array}{c}\text { No. of standard } \\
\text { exceedances }\end{array}$ & $\begin{array}{c}\text { Minimum } \\
\text { date }\end{array}$ & $\begin{array}{c}\text { Maximum } \\
\text { date }\end{array}$ \\
\hline \multicolumn{6}{|c|}{ Gore Creek subwatershed } \\
\hline Chloride & 11 & 506 & 0 & -- & -- \\
\hline \multicolumn{6}{|c|}{ Upper Eagle subwatershed } \\
\hline Chloride & 2 & 570 & 0 & -- & -- \\
\hline \multicolumn{6}{|c|}{ Middle Eagle subwatershed } \\
\hline Chloride & 0 & 504 & 2 & Feb. 24, 1977 & Sept. 4, 2002 \\
\hline \multicolumn{6}{|c|}{ Lower Eagle subwatershed } \\
\hline Chloride & 0 & 1,050 & 1 & Feb. 24, 1977 & Feb. 24, 1977 \\
\hline
\end{tabular}

Table 14. Comparison of cation and anion concentration, Gore Creek subwatershed, Colorado, 1947-2007.

[mg/L, milligrams per liter; --, no data; $\mathrm{Ca}^{2+}$, calcium; $\mathrm{Mg}^{2+}$, magnesium; $\mathrm{Na}^{+}$, sodium; $\mathrm{K}^{+}$, potassium; $\mathrm{CO}_{3}^{2-}$, carbonate; $\mathrm{SO}_{4}^{2-}, \mathrm{sulfate}^{2} \mathrm{Cl}^{-}$, chloride; $\mathrm{F}^{-}$, floride; $\mathrm{SiO}_{2}^{2-}$, silicate]

\begin{tabular}{|c|c|c|c|c|c|}
\hline Ion & $\begin{array}{l}\text { Trend slope, } \\
\text { mg/L per year }\end{array}$ & $\begin{array}{l}\text { Atomic } \\
\text { mass }\end{array}$ & $\begin{array}{c}\text { Abundance of ions } \\
\text { relative to potassium }\end{array}$ & $\begin{array}{l}\text { lonic } \\
\text { charge }\end{array}$ & $\begin{array}{l}\text { Milliequivalents } \\
\text { relative to potassium }\end{array}$ \\
\hline \multicolumn{6}{|c|}{ Cations } \\
\hline Calcium $^{1}$ & -- & 40.078 & -- & $\mathrm{Ca}^{2+}$ & -- \\
\hline Magnesium & 0.1 & 24.305 & 5.4 & $\mathrm{Mg}^{2+}$ & 10.8 \\
\hline Sodium & .3 & 22.99 & 17.0 & $\mathrm{Na}^{+}$ & 17.0 \\
\hline \multirow[t]{2}{*}{ Potassium } & .03 & 39.098 & 1.0 & $\mathrm{~K}^{+}$ & 1.0 \\
\hline & & & & Total & 28.8 \\
\hline \multicolumn{6}{|c|}{ Anions } \\
\hline Carbonate & No trend & 60.0089 & 0 & $\mathrm{CO}_{3}{ }^{2-}$ & 0 \\
\hline Sulfate & No trend & 96.0626 & 0 & $\mathrm{SO}_{4}^{2-}$ & 0 \\
\hline Silicate & No trend & 60.0843 & 0 & $\mathrm{SiO}_{2}^{2-}$ & 0 \\
\hline Chloride & 1.07 & 35.453 & 39.3 & $\mathrm{Cl}^{-}$ & 39.3 \\
\hline \multirow[t]{2}{*}{ Fluoride } & No trend & 18.9984 & 0 & $\mathrm{~F}^{-}$ & 0 \\
\hline & & & & Total & 39.3 \\
\hline
\end{tabular}

${ }^{1}$ Trend slope for calcium not reported because $\mathrm{p}$-value is between $0.05-0.15$, a weakly significant trend.

${ }^{2}$ Obtained by dividing the trend slope by the atomic mass and normalizing results for cations and anions to the smallest value (potassium, 0.0008 ).

chloride needs to be equal or greater than the sum of the milliequivalents of all cations with an upward trend at site 160 . The quantity of increase in chloride at site 160 can account for all of the anions needed to balance magnesium, sodium, and potassium with a small amount of additional chloride remaining (table 14). This excess chloride may be related to the weakly significant trend observed in calcium at site 160 .

Comparison of the chloride salts to minerals commonly found in the geologic formations within the Gore Creek subwatershed provides evidence that the trend source may not be natural. Chloride salts are ionic solids that are highly soluble and primarily are found in nature as evaporite deposits. Evaporite deposits occur in the ERW primarily in the Eagle Valley Evaporite. These evaporite deposits occur in areas downstream from Edwards, but do not appear to be present in the Gore Creek watershed (fig. 4) (Tweto, 1979). Additionally, if evaporite deposits were the source for the detected trends, it is likely that there would be an upward trend in sulfates in addition to chloride salts because of the abundance of gypsum and anhydrite (calcium-sulfate minerals) within the Eagle Valley Evaporite (Tweto, 1979). A potential source for the observed chloride salts may be the chemical anti-icing and deicing products used during winter road maintenance in municipal areas and on Interstate-70. Various magnesium chloride $\left(\mathrm{MgCl}_{2}\right)$ deicers and anti-icers, along with solid deicers $(\mathrm{NaCl}$, $\mathrm{MgCl}_{2}, \mathrm{KCl}, \mathrm{CaCl}_{2}$, and traction sand) commonly are used by the Colorado Department of Transportation (CDOT) to assist in winter road maintenance operations in Colorado (Colorado Department of Transportation, 2008). Application of these anti-icing and deicing products may explain the upward trend detected at site 160 .

Potential effects on water quality, aquatic life, and riparian vegetation are numerous and variable and beyond the scope of this study. If the present trends are the result of winter-road 
maintenance operations, then the timing and instream concentration are likely to occur in a highly variable pattern both spatially and temporally because salt contribution to the stream from these types of sources would likely coincide with the episodic melt cycles of the snow along the roadways periphery. Additionally, the pattern and concentrations of these salts in the stream also could be affected by the transient storage of an unquantified amount of these salts in the shallow groundwater system in municipal areas and roadways. These variations in concentration currently (2009) are not well represented by the data collection within the Gore Creek watershed.

\section{Nutrients}

Nutrients provide food to plants and animals but excessive concentrations in streams can have adverse effects to both biota and human health. Nitrogen and phosphorous occur naturally in streams at "background" levels, even in pristine watersheds (table 15). It is well established that a variety of watershed land uses alter the amounts of nutrients reaching surface waters.

Nitrogen and phosphorus are two nutrients that can be present in varying chemical forms within the watershed. Nitrogen species include ammonia, nitrate, nitrite, and other compounds; phosphorus species include orthophosphate and other compounds. The chemical form of these nutrients can be related to the bioavailability of the compound to an organism or how the compounds are transported in the environment. Eutrophication of streams or other waterbodies is possible when excessive concentrations of nitrogen or phosphorus are present, resulting in algae growth and decomposition that can affect the availability of dissolved oxygen for aquatic communities (Center for Disease Control and Prevention, 2011). Algal blooms can result in an initial increase in dissolved oxygen followed by decay and decomposition, which lead to decreases in dissolved oxygen that can result in a decline in the health and diversity of invertebrates and fish. Eutrophication of source waters can cause associated problems such as clogging water intakes, habitat alteration, toxic algal blooms, reduction in biodiversity, pH fluctuations, and taste-and-odor, among others (Center for Disease Control and Prevention, 2011). Additionally, elevated concentrations of un-ionized ammonia $\left(\mathrm{NH}_{3}\right)$ in a stream can be toxic to fish (Fairchild and others, 2002).

In the ERW, natural sources of nutrients (nitrogen and phosphorus) to streams may include chemical dissolution or reaction with nitrogen- and phosphorus-rich minerals within soils and geologic formations, decomposition of organic matter, and atmospheric deposition. Additional sources may include human activities related to agriculture such as fertilizers, phosphate detergents, effluent from wastewater treatment plants, seepage from septic systems, and fossil-fuel combustion. Nitrate is the most stable nitrogen species found in natural waters and can exist in a wide range of environmental conditions (Mueller and others, 1995). Nitrite is an intermediate form of nitrogen that is unstable in most natural waters. High concentrations of nitrite can be associated with untreated sewage, fertilizers, and (or) other organic waste such as decaying plant debris. Phosphate is the only significant form of dissolved phosphorus in natural water (Hem, 1989) and it is only moderately soluble. Phosphorus often adsorbs to the surface of sediment and organic particles and when found in waterways, can indicate that erosion and sediment transport is occurring (Mueller and others, 1995).

Nutrients in the ERW have been measured at 114 sites from February 21, 1968, through September 30, 2007. Median nutrient concentrations within the ERW were compared to regional reference values (table 15). The data analysis throughout this report did not differentiate if a sampling site was within a mixing zone as defined by CDPHE (Colorado Department of Public Health and Environment, 2002).

Table 15. Median nutrient concentrations for selected sites in the Eagle River watershed, Colorado, 1968-2007 and background regional reference values.

[mg/L, milligrams per liter; --, no data; <, less than]

\begin{tabular}{lcc}
\hline \multicolumn{1}{c}{ Site name (sample dates) } & $\begin{array}{c}\text { Nitrate } \\
\text { concentration, } \\
\text { mg/L }\end{array}$ & $\begin{array}{c}\text { Total phosphorous } \\
\text { concentration, } \\
\text { mg/L }\end{array}$ \\
\hline Gore Creek background ${ }^{1}(1968-1997)$ & 0.11 & $<0.01$ \\
Gore Creek at the mouth site $160(1996-2007)$ & .657 & .088 \\
Eagle River at Red Cliff site 3 (1996-2007) & -- & .004 \\
Eagle River at Avon site 173 (1996-2007) & .282 & .037 \\
Eagle River at Gypsum site 224 (1996-2007) & .585 & .088 \\
Colorado Rockies Ecoregion 21 regional reference values ${ }^{2}(2000)$ & $<.10$ & .02 \\
\hline${ }^{1}$ Wynn and others (2001). & & \\
${ }^{2}$ U.S. Environmental Protection Agency, Nutrient criteria technical guidance manual: rivers and streams. Report \\
No. EPA-822B-00-002 (2000).
\end{tabular}


Concentrations of nitrate ranged from less than 0.02 to $84 \mathrm{mg} / \mathrm{L}$ depending on location and time of year. The $25 \mathrm{th}$, 50th, and 75th percentiles for the ERW are 0.2, 0.6, and $1.2 \mathrm{mg} / \mathrm{L}$, respectively. Median concentrations for the subwatersheds ranged from $0.2 \mathrm{mg} / \mathrm{L}$ in Gore Creek subwatershed, $0.06 \mathrm{mg} / \mathrm{L}$ in the Upper Eagle subwatershed, to $0.5 \mathrm{mg} / \mathrm{L}$ in the Middle Eagle subwatershed, and $0.2 \mathrm{mg} / \mathrm{L}$ in the Lower Eagle subwatershed (table 16).

Concentrations of nitrite ranged from less than 0.01 to $1.8 \mathrm{mg} / \mathrm{L}$ depending on location and time of year. The $25 \mathrm{th}$, 50th, and 75th percentiles for the ERW are 0.002, 0.004, and $0.01 \mathrm{mg} / \mathrm{L}$, respectively. Median concentrations for the subwatersheds ranged from $0.004 \mathrm{mg} / \mathrm{L}$ in Gore Creek subwatershed, $0.003 \mathrm{mg} / \mathrm{L}$ in the Upper Eagle subwatershed, $0.006 \mathrm{mg} / \mathrm{L}$ in the Middle Eagle subwatershed, to $0.003 \mathrm{mg} / \mathrm{L}$ in the Lower Eagle subwatershed (table 16).

Concentrations of ammonia ranged from less than 0.1 to $18.3 \mathrm{mg} / \mathrm{L}$ depending on location and time of year. The 25th, 50th, and 75th percentiles for the ERW are 0.01, 0.02, and $0.05 \mathrm{mg} / \mathrm{L}$, respectively. Median concentrations for the subwatersheds were $0.02 \mathrm{mg} / \mathrm{L}$ in Gore Creek subwatershed, $0.03 \mathrm{mg} / \mathrm{L}$ in the Upper Eagle subwatershed, $0.03 \mathrm{mg} / \mathrm{L}$ in the Middle Eagle subwatershed, and $0.04 \mathrm{mg} / \mathrm{L}$ in the Lower Eagle subwatershed (table 16).

Concentrations of un-ionized ammonia ranged from less than 0.01 to $1.47 \mathrm{mg} / \mathrm{L}$ depending on location and time of year. The 25th, 50th, and 75th percentiles for the ERW are $0.0003,0.001$, and $0.004 \mathrm{mg} / \mathrm{L}$, respectively. Median concentrations for the subwatersheds ranged from $0.001 \mathrm{mg} / \mathrm{L}$ in Gore Creek subwatershed, $0.001 \mathrm{mg} / \mathrm{L}$ in the Upper Eagle subwatershed, to $0.003 \mathrm{mg} / \mathrm{L}$ in the Middle Eagle subwatershed (table 16).

Concentrations of total phosphorus ranged from less than 0.05 to $17 \mathrm{mg} / \mathrm{L}$ depending on location and time of year. The 25th, 50th, and 75th percentiles for the ERW are 0.02, 0.04, and $0.1 \mathrm{mg} / \mathrm{L}$, respectively. Median concentrations for the subwatersheds ranged from $0.05 \mathrm{mg} / \mathrm{L}$ in Gore Creek subwatershed, $0.01 \mathrm{mg} / \mathrm{L}$ in the Upper Eagle subwatershed, $0.06 \mathrm{mg} / \mathrm{L}$

Table 16. Summary statistics of nutrients data in the Eagle River watershed, Colorado, by subwatershed.

[No., number; MRL, Minimum Reporting Limit; <, less than; --, undetermined]

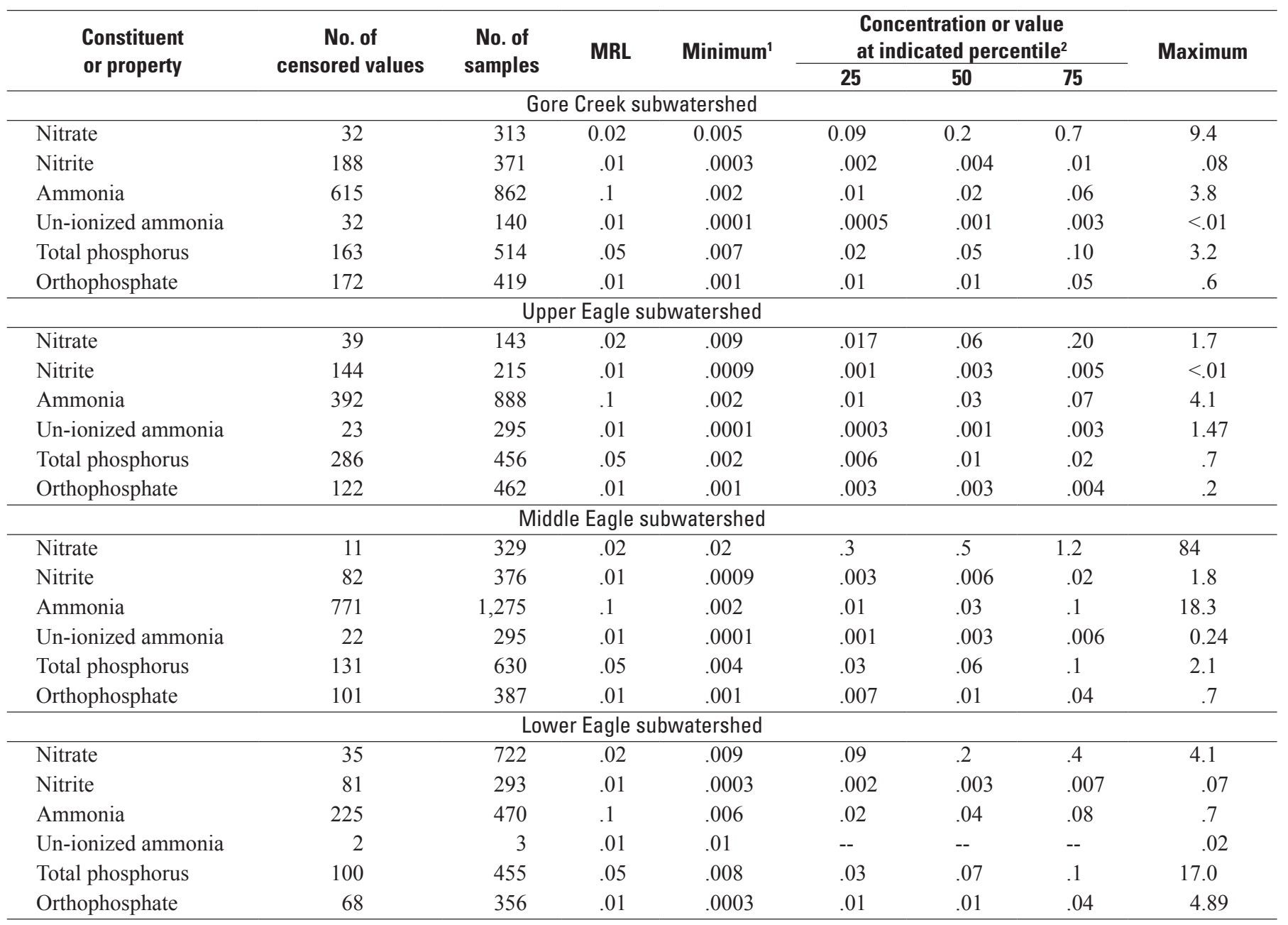

${ }^{1}$ Uncensored values.

${ }^{2}$ Percentile values can be less than the MRL values due to multiple MRLs within the combined dataset. 
in the Middle Eagle subwatershed, to $0.07 \mathrm{mg} / \mathrm{L}$ in the Lower Eagle subwatershed (table 16).

Concentrations of orthophosphate ranged from less than 0.01 to $4.89 \mathrm{mg} / \mathrm{L}$ depending on location and time of year. The 25th, 50th, and 75th percentiles for the ERW are 0.003, 0.006 , and $0.03 \mathrm{mg} / \mathrm{L}$, respectively. Median concentrations for the subwatersheds ranged from $0.01 \mathrm{mg} / \mathrm{L}$ in Gore Creek subwatershed, $0.003 \mathrm{mg} / \mathrm{L}$ in the Upper Eagle subwatershed, $0.01 \mathrm{mg} / \mathrm{L}$ in the Middle Eagle subwatershed, to $0.01 \mathrm{mg} / \mathrm{L}$ in the Lower Eagle subwatershed (table 16).

The spatial pattern of nutrients (figs. 46-50) shows lower concentrations on many tributaries and on the Eagle River upstream from Red Cliff with increases in nutrients downstream of major urban areas. Seasonal variations show that for many nutrient species, concentrations tend to be lowest May-June and highest January-March (figs. 51-54). The gradual changes in concentrations between seasons may be related to dilution effects from increases and decreases in streamflow. Downstream in the Eagle River near Gypsum (site 224), total phosphorus concentrations are elevated January-May and July-September with concentrations highest in April. These higher concentrations may be related to sediment transported by snowmelt runoff (January-April) and rainfall (July-September).

Water-quality State standards have been established by CDPHE for nitrate $(10 \mathrm{mg} / \mathrm{L})$, nitrite $(0.05 \mathrm{mg} / \mathrm{L})$, and un-ionized ammonia (TVS) for selected stream segments in the watershed (Colorado Department of Public Health and Environment, 2007) (fig. 12). CDPHE has not established an in-stream State standard for total phosphorus. The State of Colorado Water Quality Control Division currently (2009) is working to understand the link between nutrient criteria for streams and their designated uses (Colorado Water Quality Forum, 2008). General recommendations for total phosphorus concentration exist from the USEPA at concentrations of 0.01 and $0.1 \mathrm{mg} / \mathrm{L}$ (U.S. Environmental Protection Agency, 1986). In this report, $0.1 \mathrm{mg} / \mathrm{L}$ was used to compare sample concentrations. This was done in recognition that this concentration is only a recommendation, not an actual standard, and because many of the method reporting limits (MRLs) used in ERW data collection are equal to or exceed the lower $0.01 \mathrm{mg} / \mathrm{L}$ recommendation.

The State standard value for chronic nitrate set by the CDPHE is $10.0 \mathrm{mg} / \mathrm{L}$ for all reaches of the ERW except for segment $11(100.0 \mathrm{mg} / \mathrm{L})($ Colorado Department of Public Health and Environment, 2008). The State standard for chronic nitrate $(10 \mathrm{mg} / \mathrm{L})$ was exceeded less than 1 percent ( 2 comparisons) of the 1,507 comparisons in the ERW (table 17).

The State standard for chronic nitrite set by the CDPHE is $0.05 \mathrm{mg} / \mathrm{L}$ for all reaches of the ERW except for segment $11(10.0 \mathrm{mg} / \mathrm{L})($ Colorado Department of Public Health and Environment, 2008). The State standard for chronic nitrite was exceeded 9 percent ( 70 comparisons) of the 1,255 comparisons in the ERW (table 17).

The State standard for un-ionized ammonia is set by the CDPHE as a TVS. The TVS are site-specific for both acute and chronic exposure levels based on $\mathrm{pH}$ and water temperature. In samples where either $\mathrm{pH}$ or water temperature was not measured, the sample was not compared to the TVS. At present, segment 11 of the ERW does not have a State standard for un-ionized ammonia (fig. 12). ERW had 7 sites with samples with exceedances of the un-ionized ammonia chronic TVS. The State standard for un-ionized ammonia was exceeded less than 5 times over a period of several years for most sites in the ERW, although some sites exhibited exceedances multiple times within a given year. Out of the 3,495 comparisons of un-ionized ammonia measurements to State standards, less than 1 percent ( 21 comparisons) of the total number of measurements exceeded the chronic State standard. The ERW had 7 sites with samples with exceedances of the acute State standard. Out of the 3,495 comparisons of un-ionized ammonia measurements to State standards, less than 1 percent (28 comparisons) of the total number of measurements exceeded the acute State standard (table 17).

The USEPA recommendation for total phosphorus is $0.1 \mathrm{mg} / \mathrm{L}$ (Colorado Department of Public Health and Environment, 2008) and is used in this report for all reaches of the ERW. The total phosphorus recommended concentration was exceeded 10 or less times over a period of several years for most sites in the ERW; approximately 24 percent (511 comparisons) of the total number of comparisons $(2,055)$ were higher than the recommended concentration (table 17). Figures 55-60 show the concentrations for total phosphorus through time at sites with numerous comparisons that were higher than the recommended concentrations.

To better represent exceedances of State standards for nutrients in the ERW as a whole, the combined number of samples with detected exceedances for any of the nutrient species discussed was made into map form. Maps summarizing the spatial extent of chronic-nutrient exceedances and acutenutrient exceedances are presented in figures 61-62.

Evaluation of temporal trends was done for all sites with nitrate data; trend analysis was done at the 3 sites that met the criteria set in the "Data Sources, Compilation, Review, and Analysis" section of this report. Findings from the trend analysis are presented in table 18 and figures 46-50. Seasonal Kendall Trend tests were done for various time periods between 1994-2007 depending on the data available at each site. A significant upward trend ( $p$-value of 0.015) in nitrate at site 224 (Lower Eagle subwatershed) occurs for 1994-2007. This trend represented a $0.30-\mathrm{mg} / \mathrm{L}$ increase over the entire specified time period. Upstream at sites 173 (Middle Eagle subwatershed) and 160 (Gore Creek subwatershed) there was no trend. The area between sites 224 (Lower Eagle subwatershed) and 173 (Middle Eagle subwatershed) contains several tributaries and urban areas including Avon, Edwards, Eagle, and Gypsum (fig. 46) which may contribute to the observed trend.

Evaluation of temporal trends was done for all sites with nitrite data; trend analysis was done at the 3 sites that met the criteria set in the "Data Sources, Compilation, Review, and Analysis" section of this report. Findings from the trend analysis are presented in table 18 and figure 47. Seasonal Kendall Trend tests were done for the time period 1999-2007. There is 


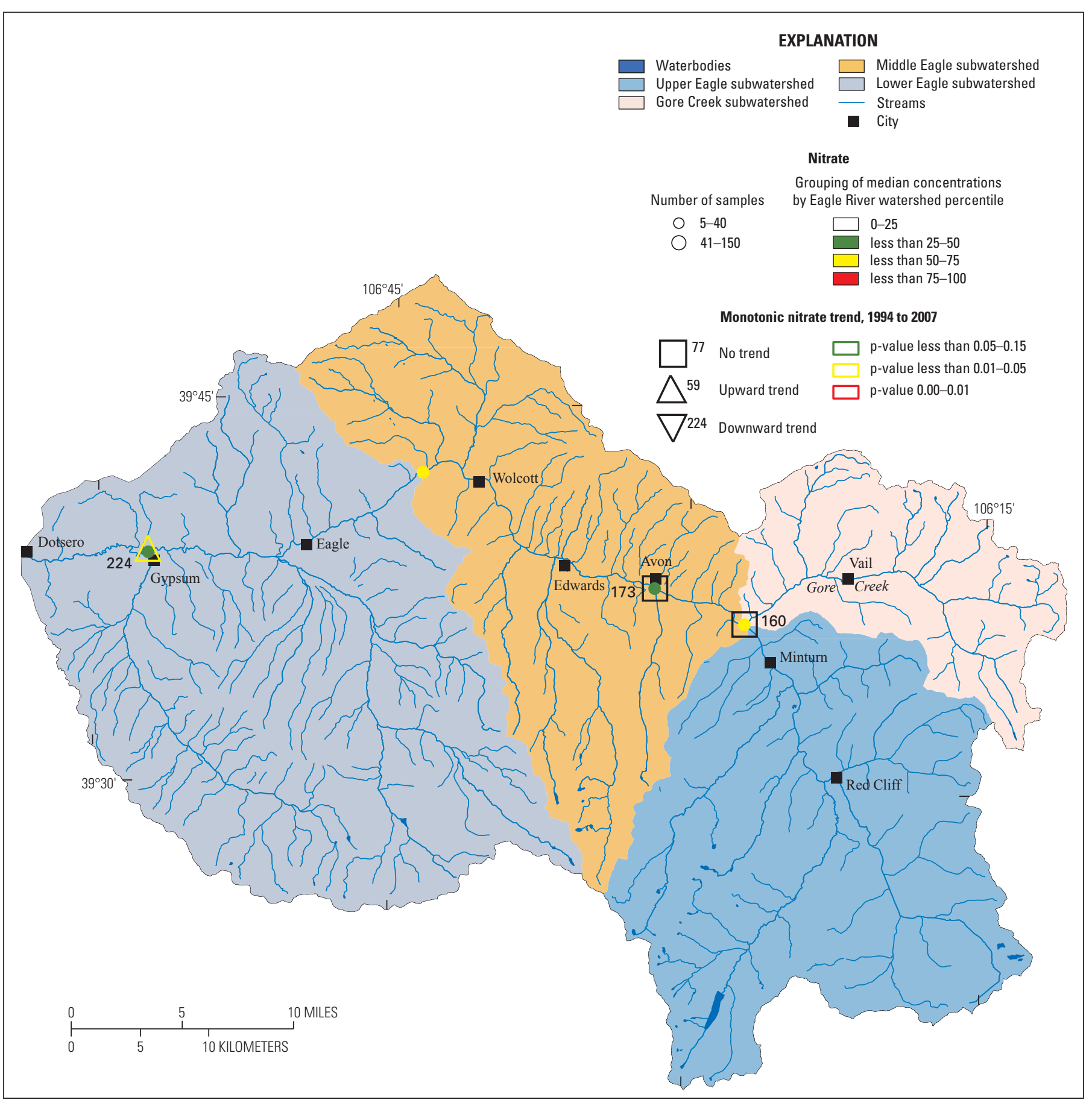

Base from U.S. Geological Survey digital data, 2007,

Universal Transverse Mercator projection

Zone 13

Figure 46. Spatial distribution of median-concentration percentiles and monotonic nitrate trends at selected sites in the Eagle River watershed, Colorado, 1995-2007. 


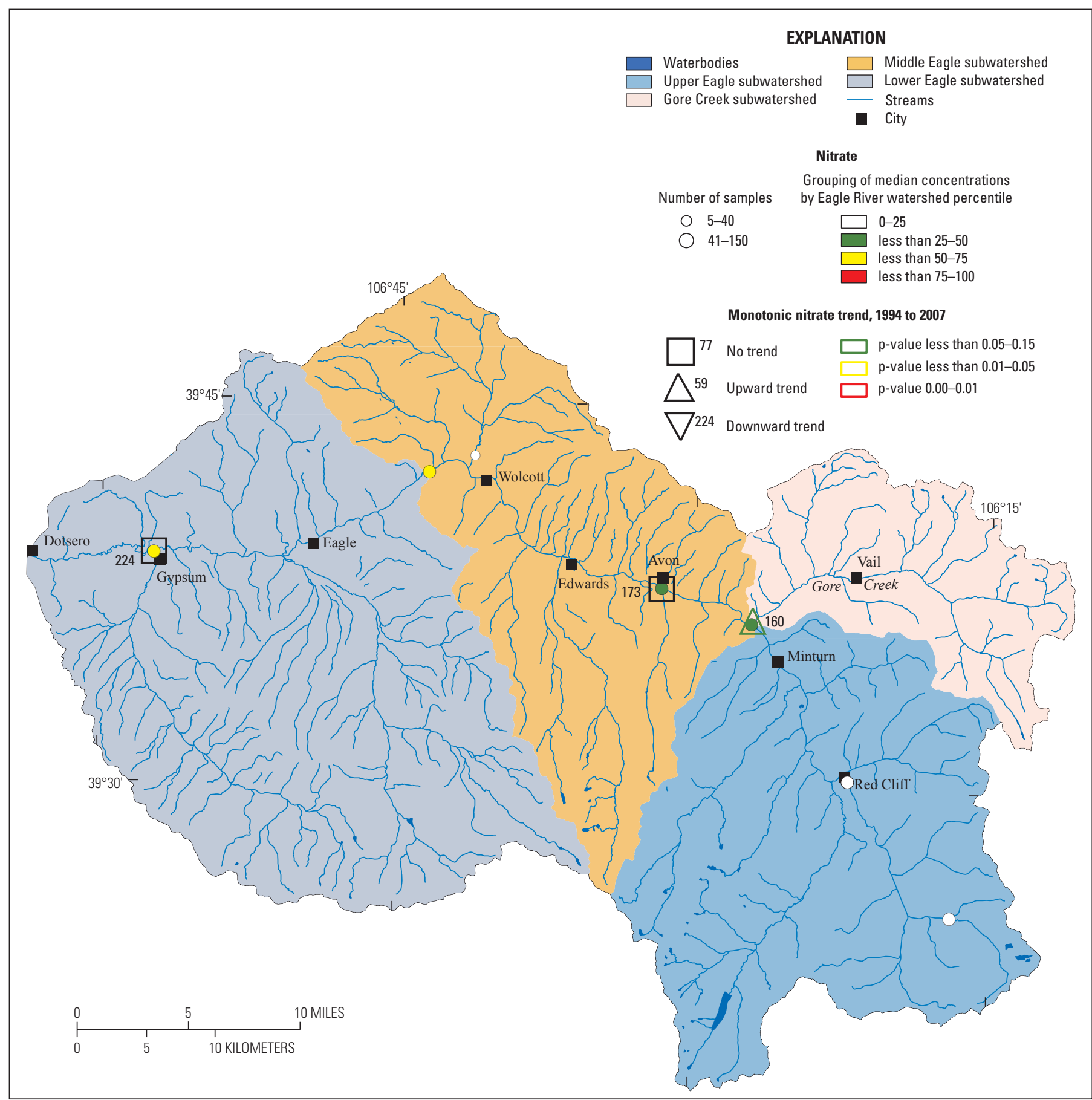

Base from U.S. Geological Survey digital data, 2007

Universal Transverse Mercator projection

Zone 13

Figure 47. Spatial distribution of median-concentration percentiles and monotonic nitrite trends at selected sites in the Eagle River watershed, Colorado, 1995-2007. 


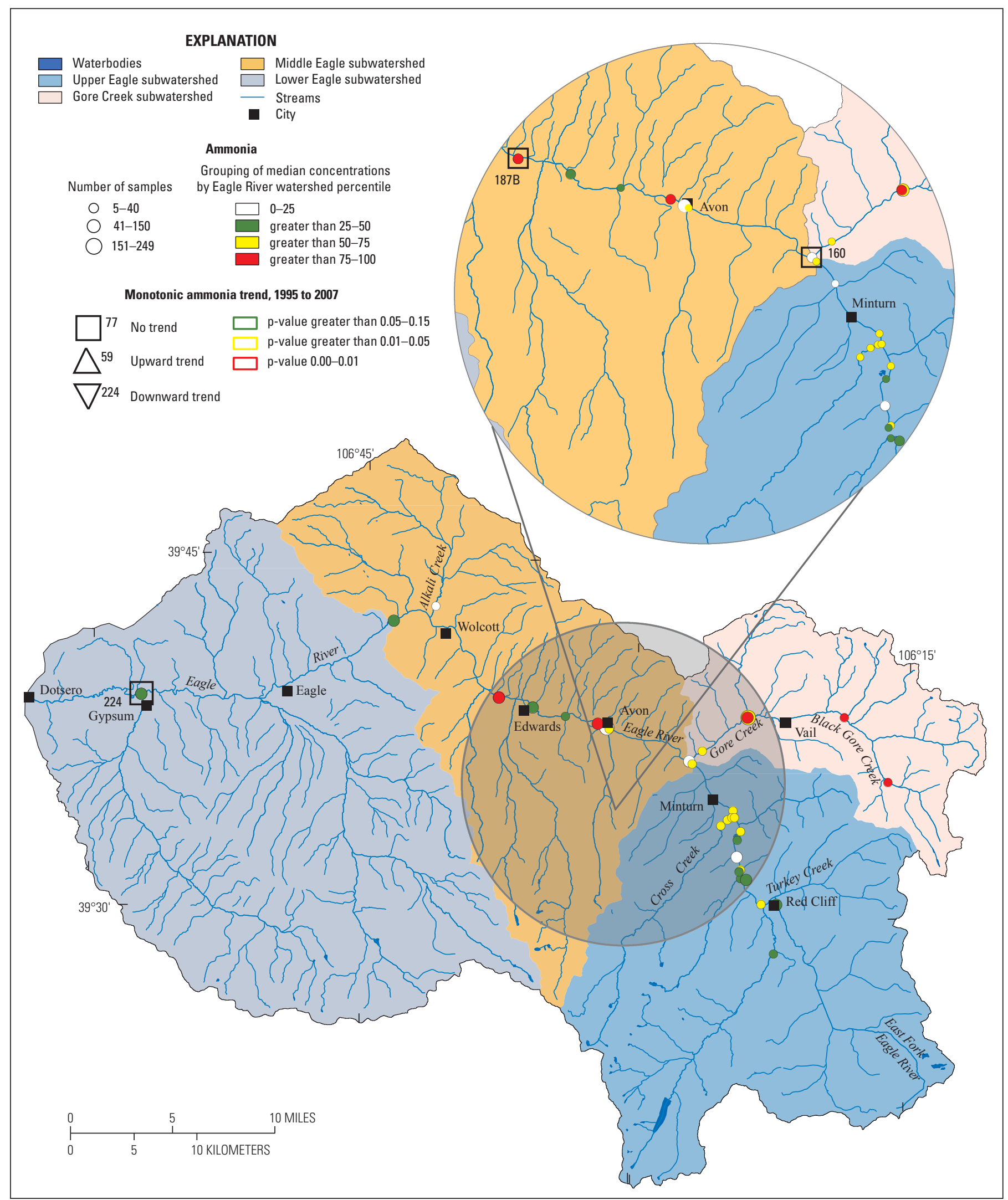

Base from U.S. Geological Survey digital data, 2007,

Universal Transverse Mercator projection

Zone 13

Figure 48. Spatial distribution of median-concentration percentiles and monotonic ammonia trends at selected sites in the Eagle River watershed, Colorado, 1995-2007. 


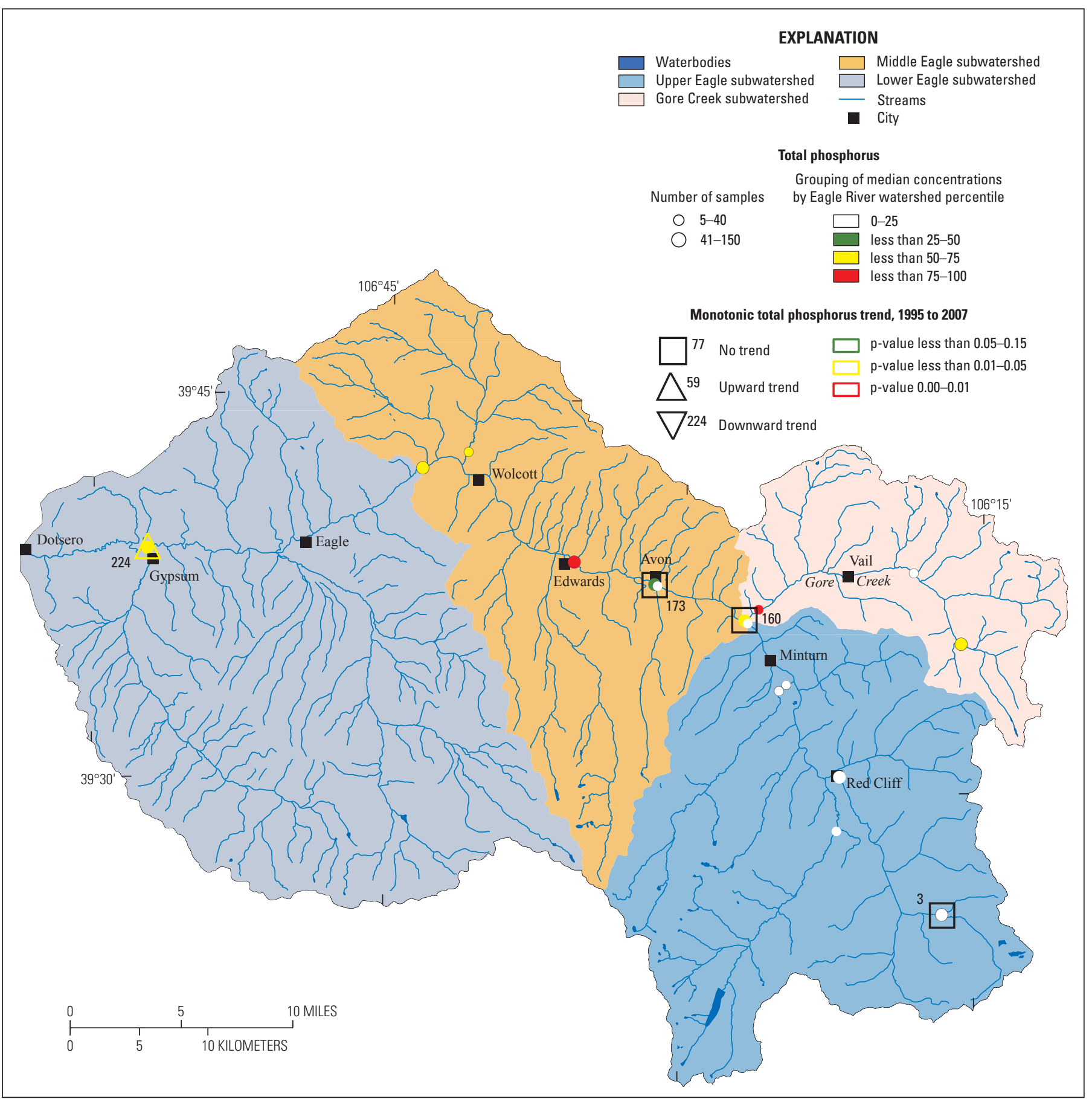

Base from U.S. Geological Survey digital data, 2007,

Universal Transverse Mercator projection

Zone 13

Figure 49. Spatial distribution of median-concentration percentiles and monotonic total phosphorus trends at selected sites in the Eagle River watershed, Colorado, 1995-2007. 


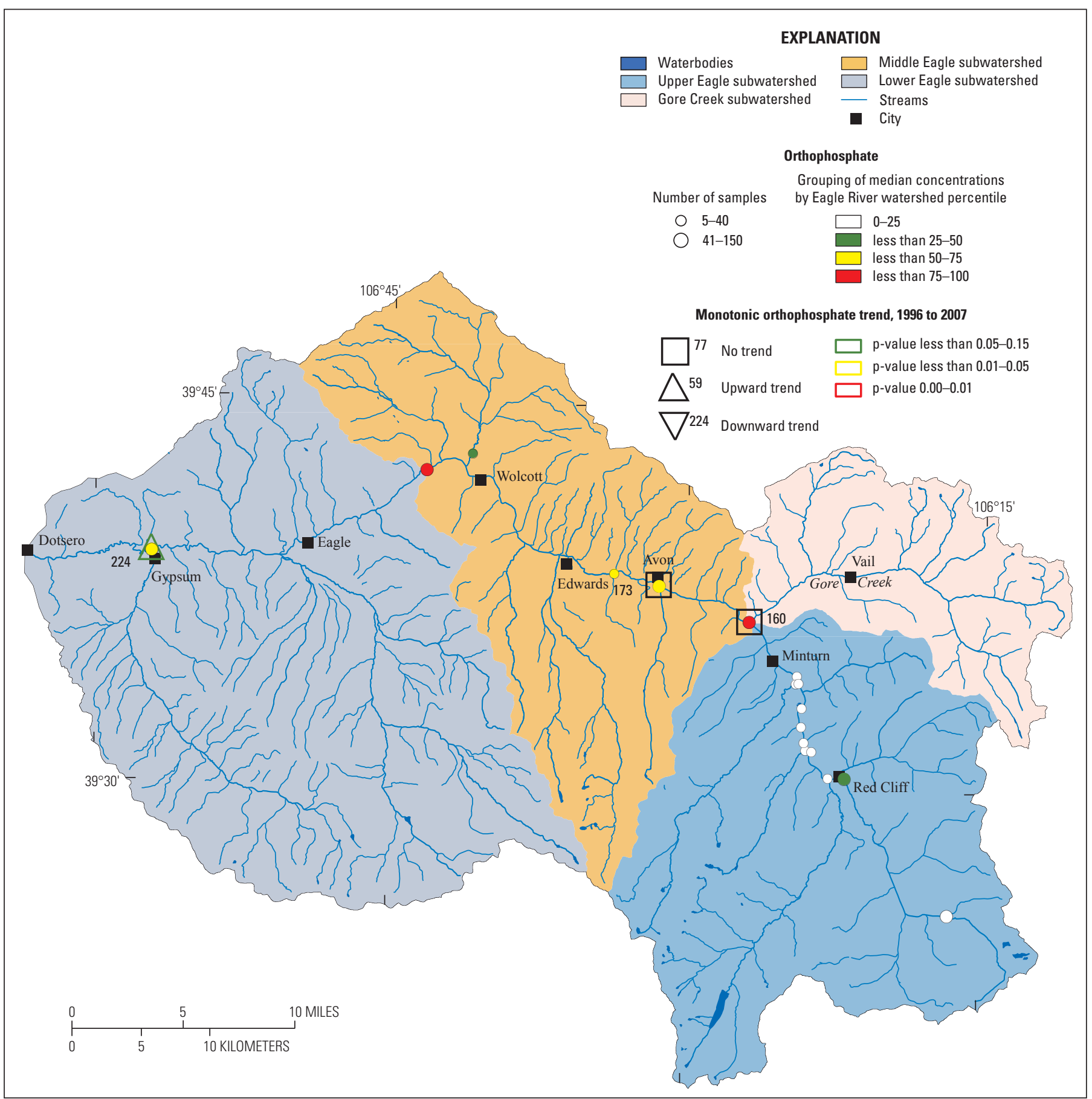

Base from U.S. Geological Survey digital data, 2007,

Universal Transverse Mercator projection

Zone 13

Figure 50. Spatial distribution of median-concentration percentiles and monotonic orthophosphate trends at selected sites in the Eagle River watershed, Colorado, 1995-2007. 


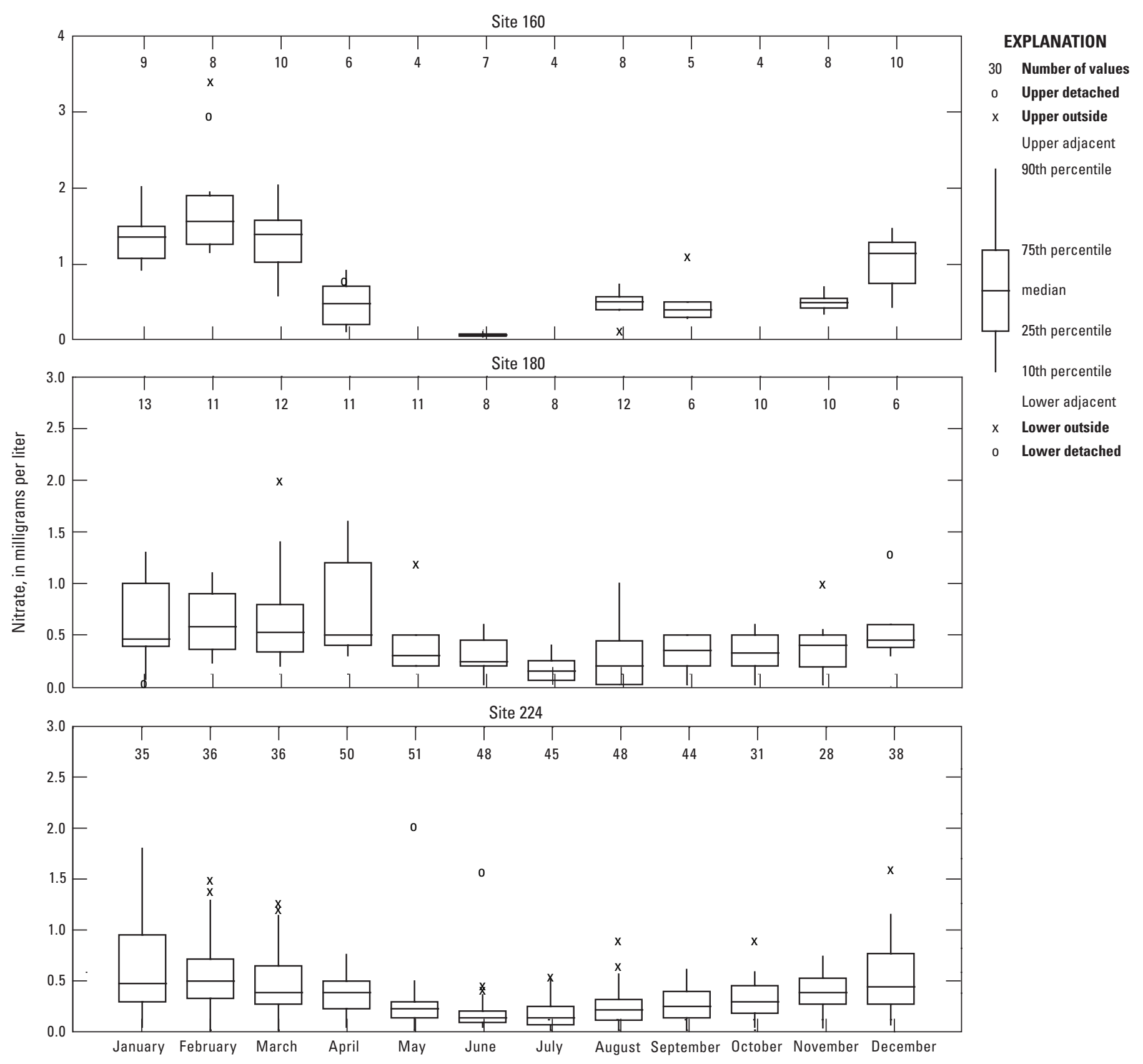

Figure 51. Seasonal patterns of nitrate concentrations at selected sites in the Eagle River watershed, Colorado, $1947-2007$. 


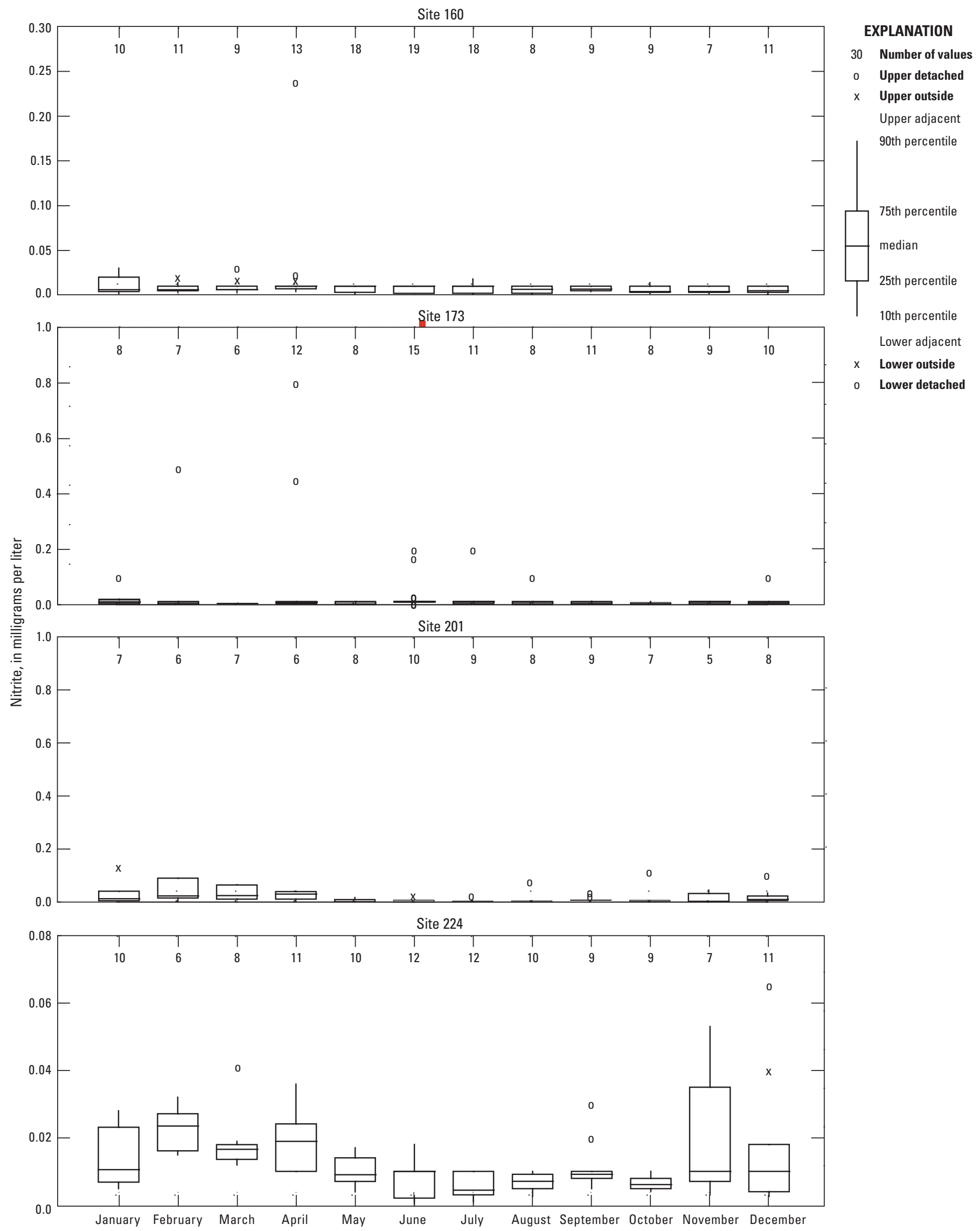

Figure 52. Seasonal patterns of nitrite concentrations at selected sites in the Eagle River watershed, Colorado, $1947-2007$. 


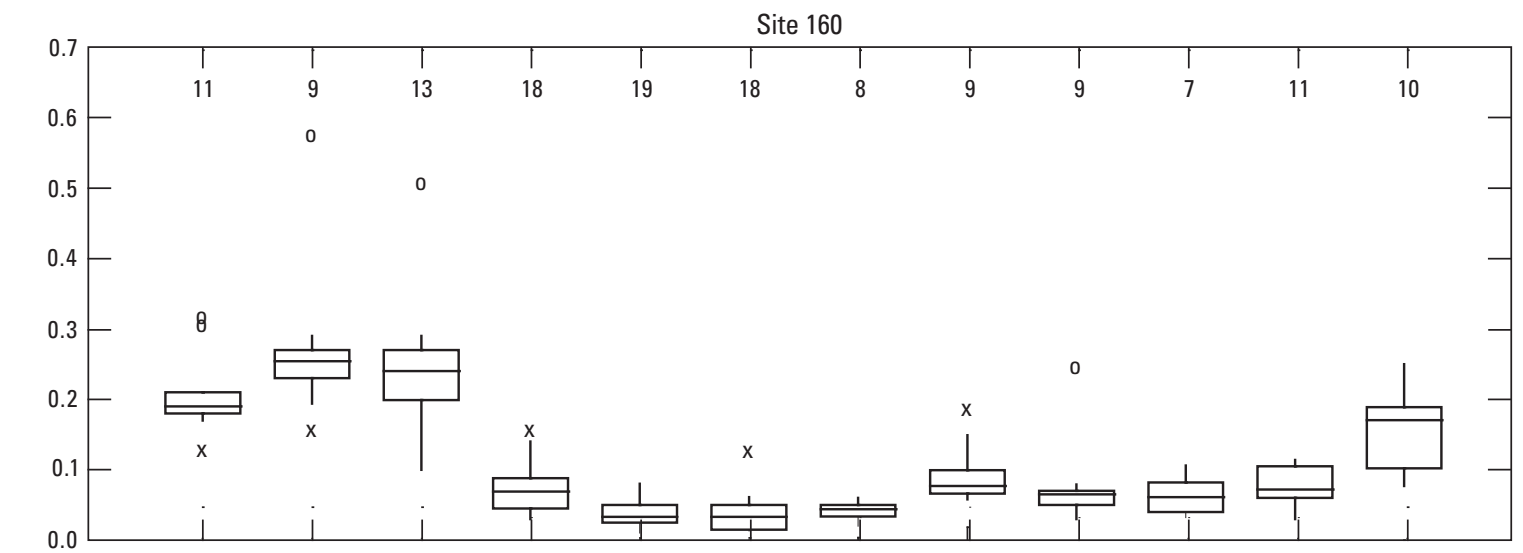

EXPLANATION

30 Number of values

o Upper detached

$x \quad$ Upper outside

Upper adjacent

90th percentile

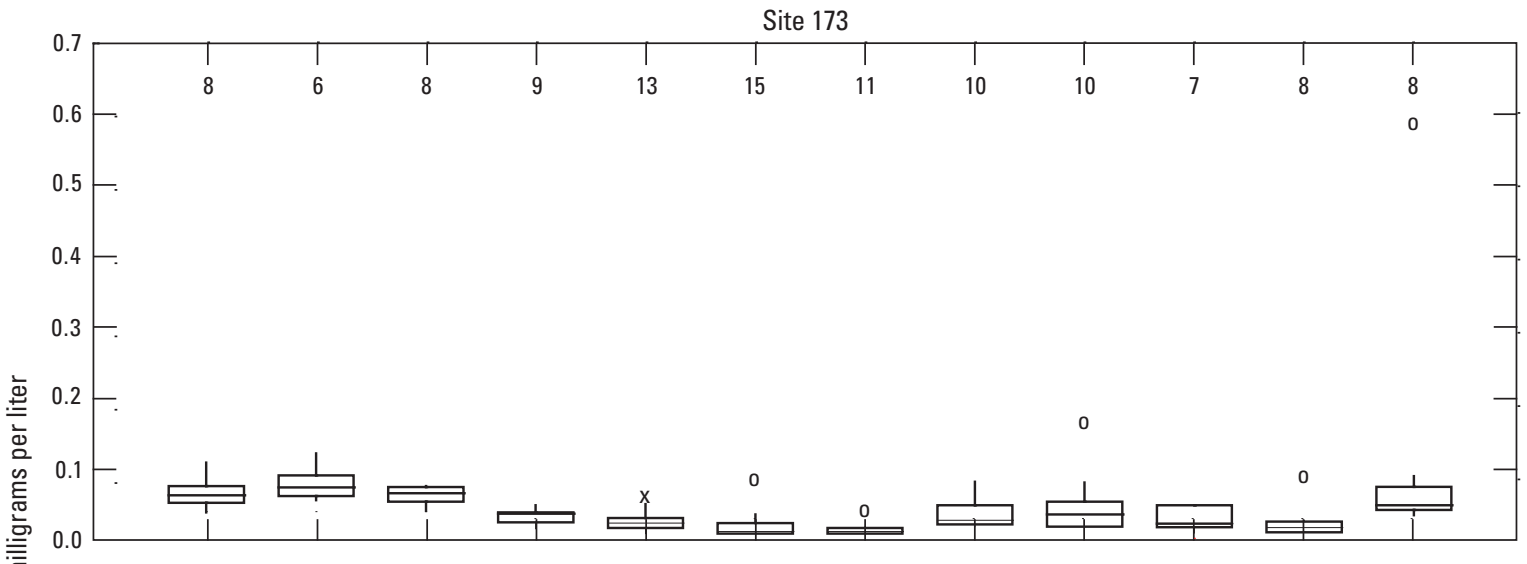

75th percentile

median

25th percentile

10th percentile

Lower adjacent

$x$ Lower outside

0 Lower detached

Site 201
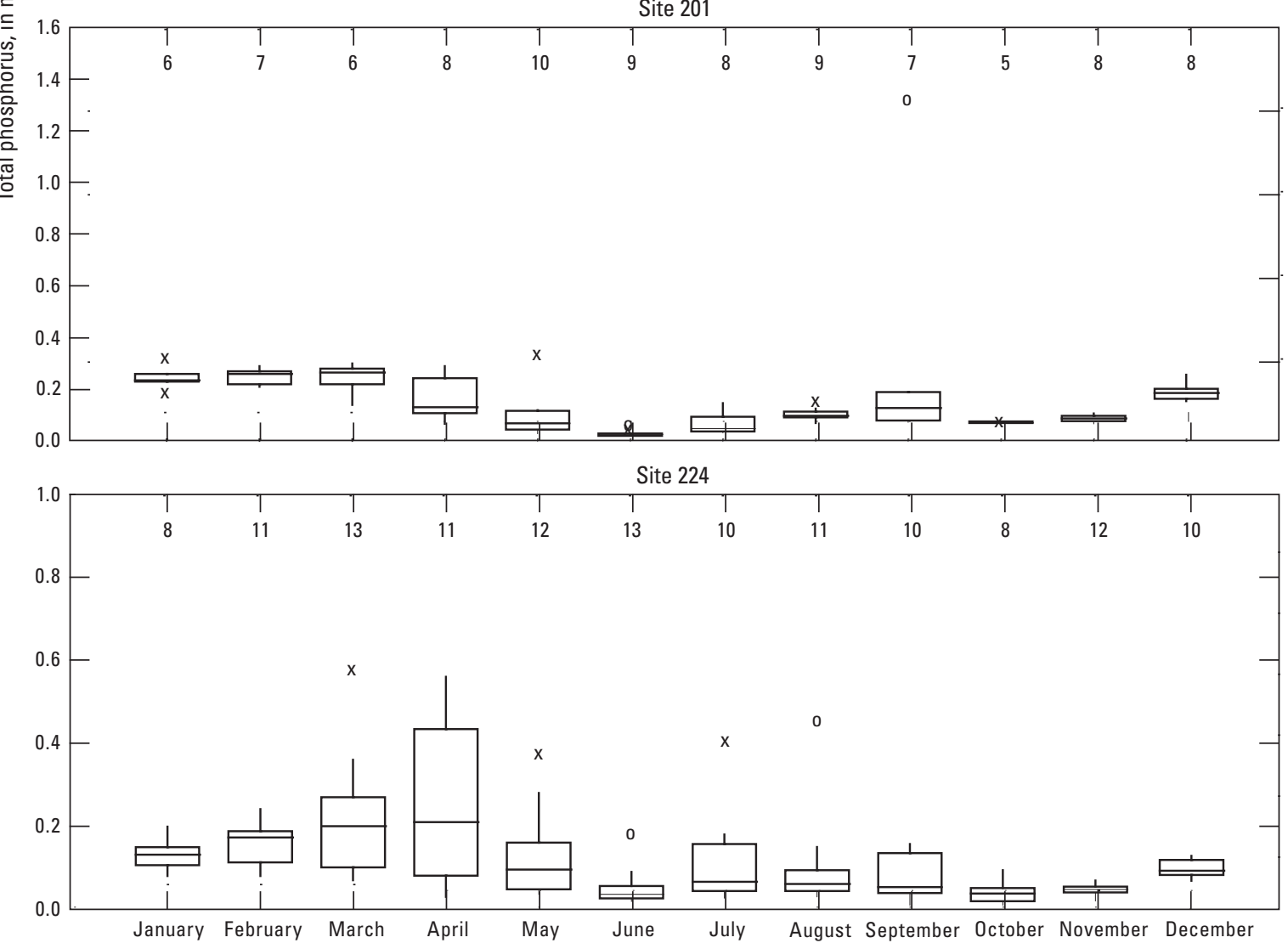

Figure 53. Seasonal patterns of total phosphorus concentrations at selected sites in the Eagle River watershed, Colorado, $1947-2007$. 


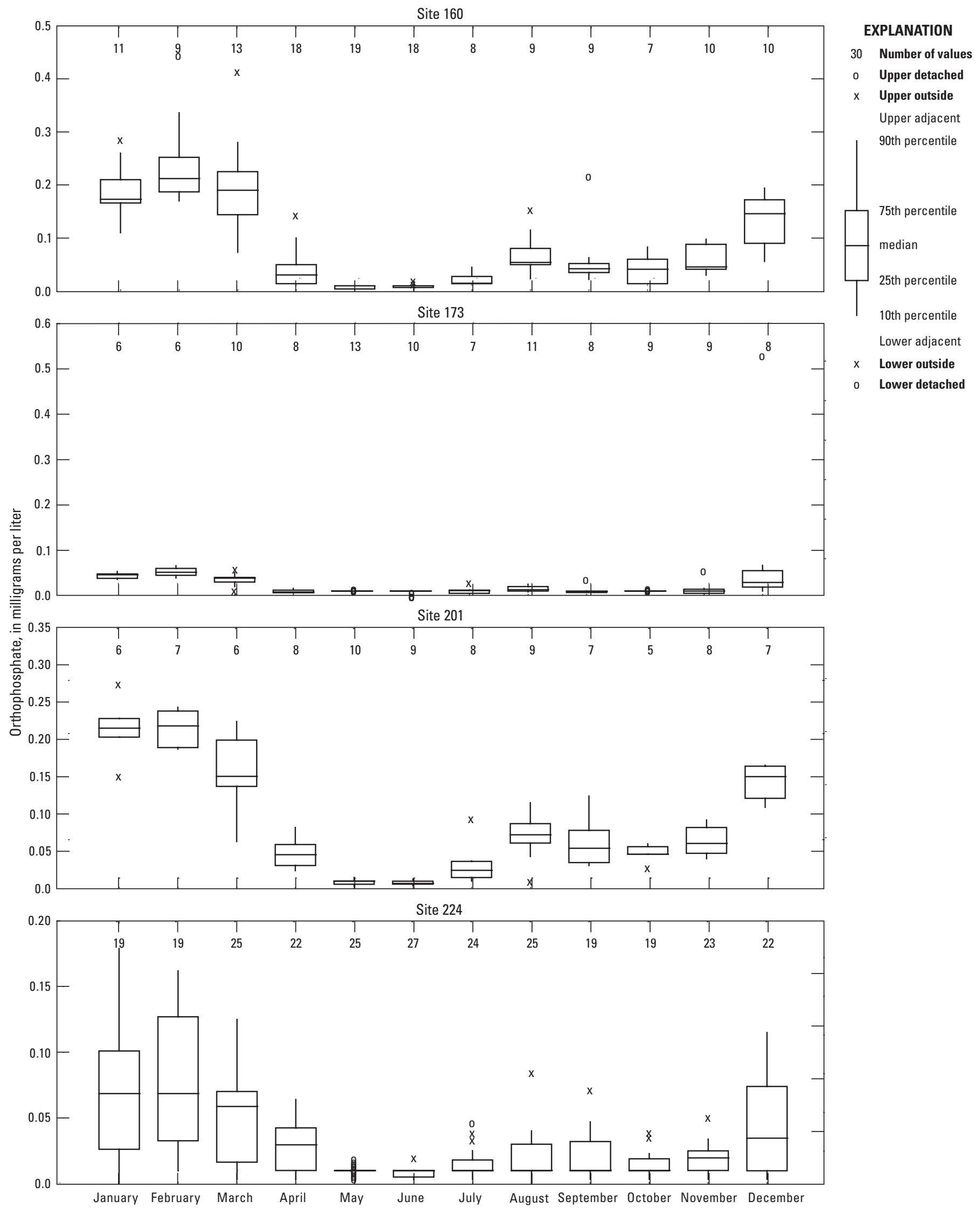

Figure 54. Seasonal patterns of orthophosphate concentrations at selected sites in the Eagle River watershed, Colorado, $1947-2007$. 
Table 17. Summary of water-quality standard or recommendation exceedances for nutrients in the Eagle River watershed by subwatershed.

[No., number; <, less than;--, no data]

\begin{tabular}{|c|c|c|c|c|c|c|}
\hline $\begin{array}{l}\text { Constituent } \\
\text { or property }\end{array}$ & $\begin{array}{l}\text { No. of } \\
\text { censored } \\
\text { values }\end{array}$ & $\begin{array}{c}\text { No. of } \\
\text { samples }\end{array}$ & $\begin{array}{c}\text { No. of standard } \\
\text { or recommendation } \\
\text { exceedances }\end{array}$ & $\begin{array}{c}\text { Percent of } \\
\text { standard } \\
\text { exceedances }\end{array}$ & $\begin{array}{l}\text { Minimum } \\
\text { date }\end{array}$ & $\begin{array}{l}\text { Maximum } \\
\text { date }\end{array}$ \\
\hline \multicolumn{7}{|c|}{ Gore Creek subwatershed } \\
\hline Nitrate, chronic & 32 & 313 & 0 & 0 & -- & -- \\
\hline Nitrite, chronic & 188 & 371 & 19 & 5 & Feb. 4, 1971 & Mar. 22, 2005 \\
\hline Un-ionized ammonia, acute & 615 & 862 & 3 & $<1$ & Mar. 12, 1970 & Feb. 19,1976 \\
\hline Un-ionized ammonia, chronic & 615 & 862 & 3 & $<1$ & Mar. 12, 1970 & Feb. 19,1976 \\
\hline Total phosphorous, chronic ${ }^{1}$ & 163 & 514 & 159 & 31 & Apr. 8, 1974 & Mar. 21, 2007 \\
\hline \multicolumn{7}{|c|}{ Upper Eagle subwatershed } \\
\hline Nitrate, chronic & 39 & 143 & 0 & 0 & -- & -- \\
\hline Nitrite, chronic & 144 & 215 & 1 & $<1$ & Oct. 27,1976 & Oct. 27,1976 \\
\hline Un-ionized ammonia, acute & 392 & 888 & 11 & 1 & Nov. 12, 1974 & Sept. 2, 1992 \\
\hline Un-ionized ammonia, chronic & 392 & 888 & 7 & $<1$ & Nov. 12, 1974 & Sept. 2, 1992 \\
\hline Total phosphorous, chronic ${ }^{1}$ & 286 & 456 & 6 & 1 & Jan. 28, 1975 & Jan. 8, 1998 \\
\hline \multicolumn{7}{|c|}{ Middle Eagle subwatershed } \\
\hline Nitrate, chronic & 11 & 329 & 2 & $<1$ & Sept. 26, 1990 & Feb. 22,1991 \\
\hline Nitrite, chronic & 82 & 376 & 48 & 13 & Sept. 26, 1990 & Feb. 19, 2003 \\
\hline Un-ionized ammonia, acute & 771 & 1,275 & 14 & 1 & Mar. 6, 1975 & Feb. 13, 2007 \\
\hline Un-ionized ammonia, chronic & 771 & 1,275 & 11 & $<1$ & Mar. 6, 1975 & Feb. 13, 2007 \\
\hline Total phosphorous, chronic ${ }^{1}$ & 131 & 630 & 203 & 32 & Jan. 6, 1970 & Dec. 5, 2007 \\
\hline \multicolumn{7}{|c|}{ Lower Eagle subwatershed } \\
\hline Nitrate, chronic & 35 & 722 & 0 & 0 & -- & -- \\
\hline Nitrite, chronic & 81 & 293 & 2 & $<1$ & Nov. 12, 2002 & Oct. 7, 2003 \\
\hline Un-ionized ammonia, acute & 225 & 470 & 0 & 0 & -- & -- \\
\hline Un-ionized ammonia, chronic & 225 & 470 & 0 & 0 & -- & -- \\
\hline Total phosphorous, chronic ${ }^{1}$ & 100 & 455 & 143 & 31 & May 16, 1974 & July 26, 2007 \\
\hline
\end{tabular}

${ }^{1}$ Water-quality recommended level set by U.S. Environmental Protection Agency (2000), no State standard. 


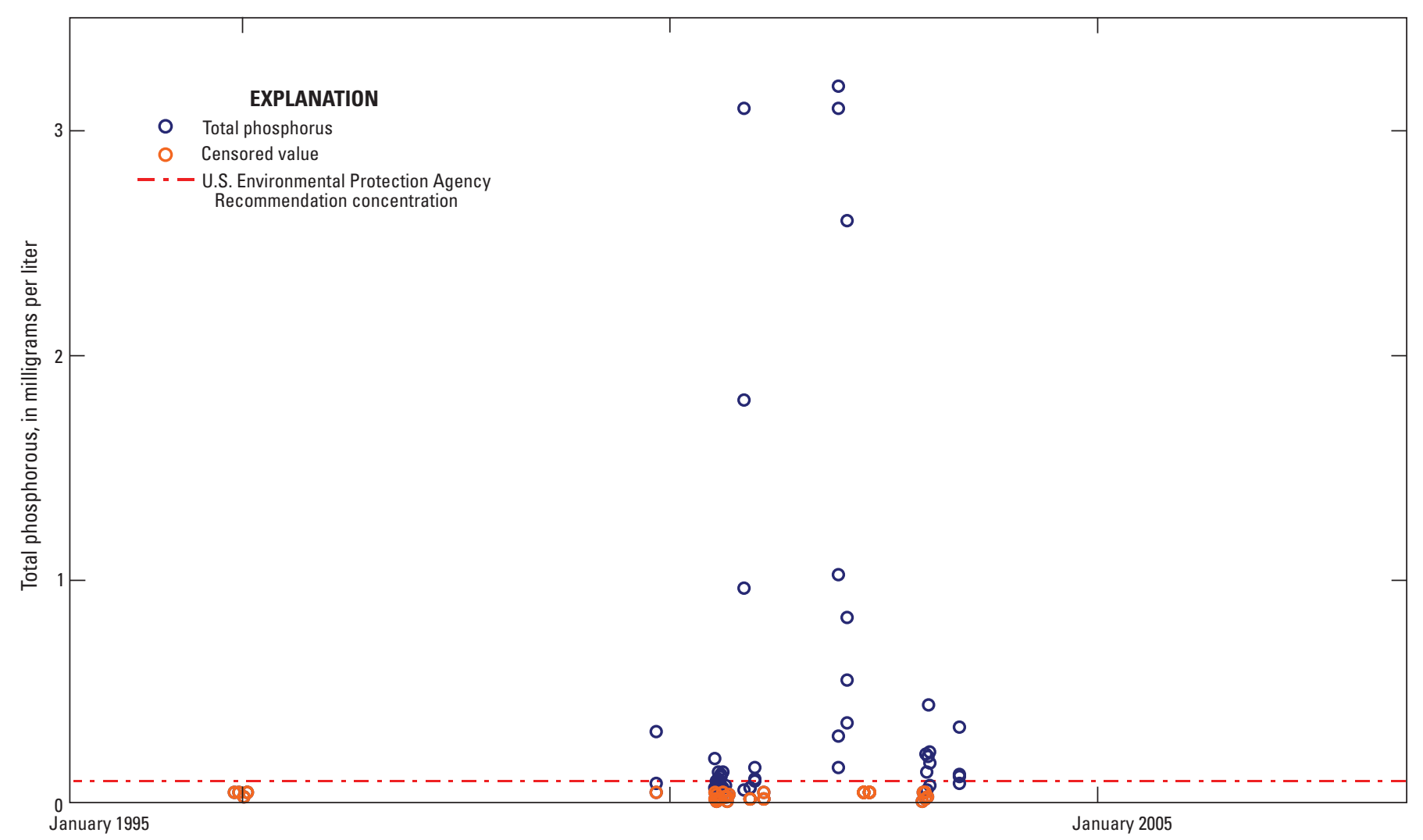

Figure 55. Temporal distribution of total phosphorus concentrations at site 110, Eagle River watershed, Colorado, $1997-2004$.

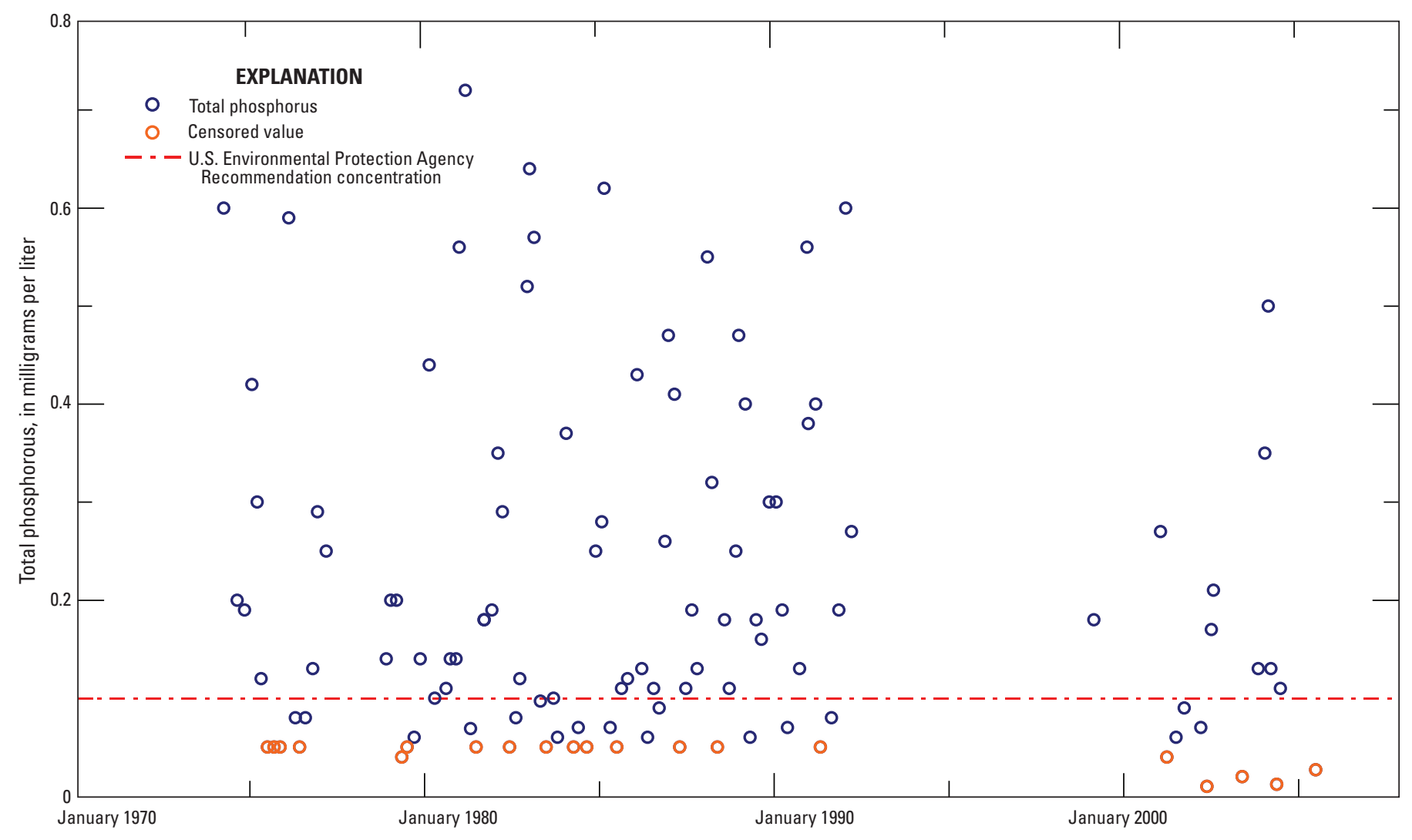

Figure 56. Temporal distribution of total phosphorus concentrations at site 157, Eagle River watershed, Colorado, 1974-2006. 


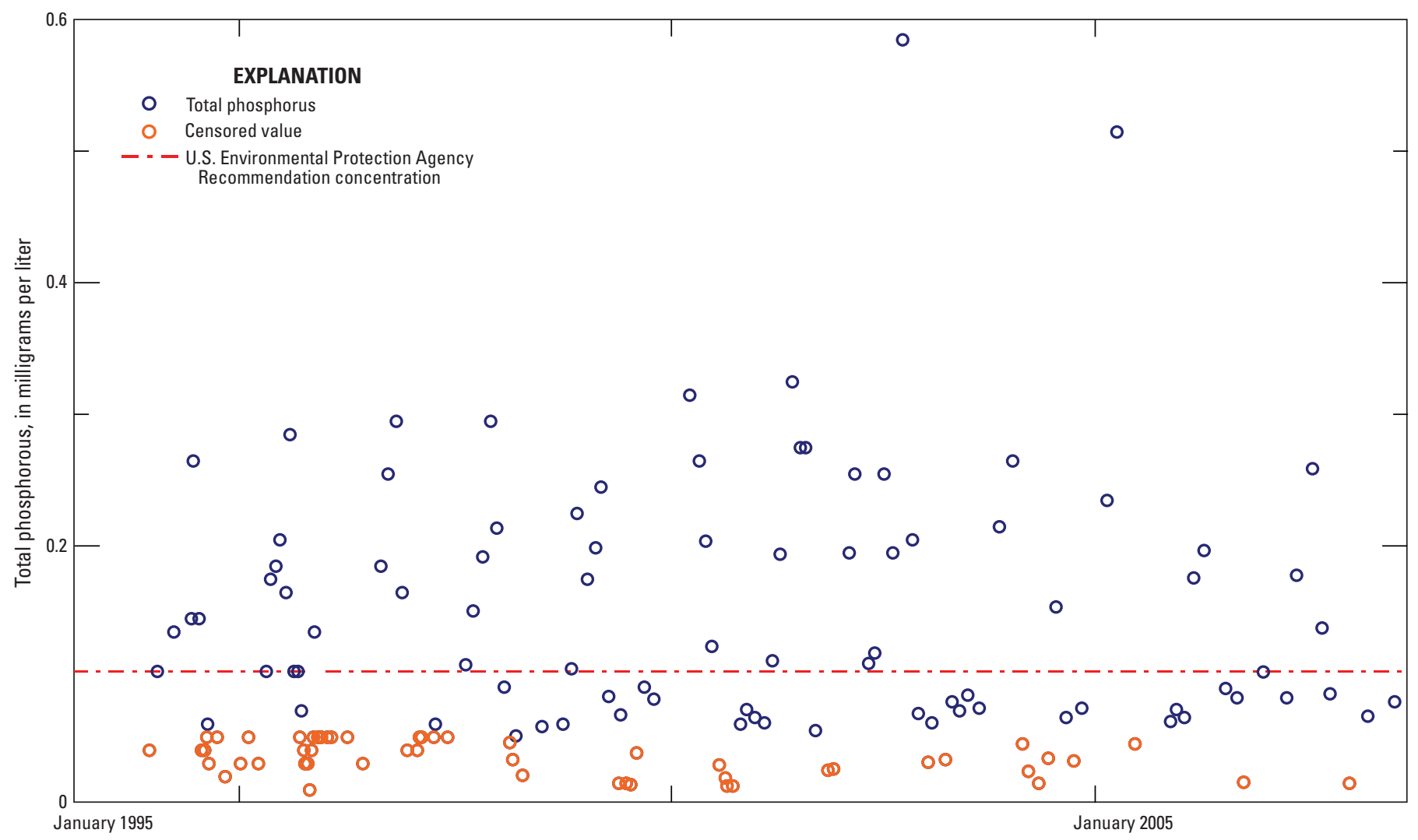

Figure 57. Temporal distribution of total phosphorus concentrations at site 160, Eagle River watershed, Colorado, $1996-2007$.

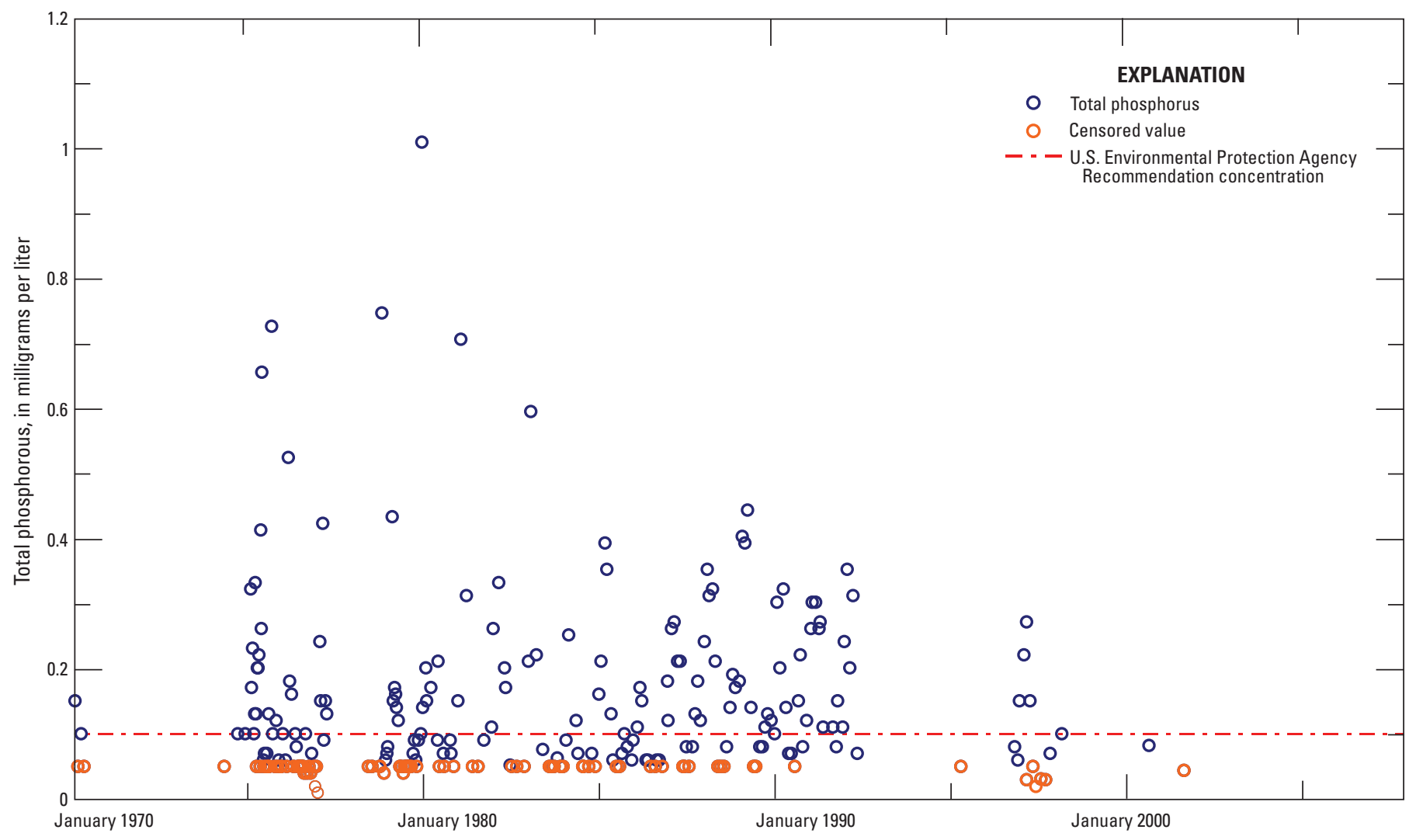

Figure 58. Temporal distribution of total phosphorus concentrations at site 180, Eagle River watershed, Colorado, 1970-2002. 


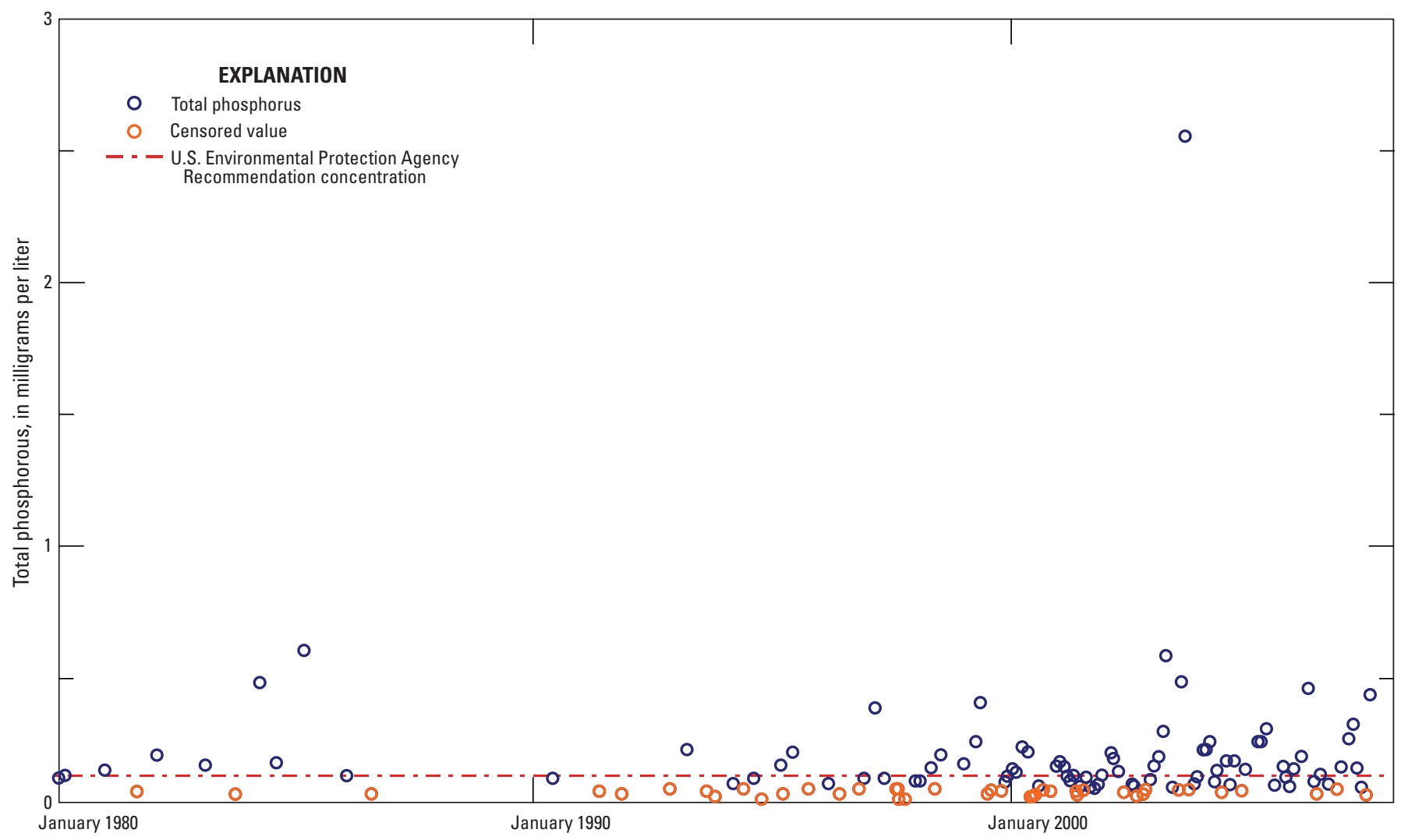

Figure 59. Temporal distribution of total phosphorus concentrations at site 224, Eagle River watershed, Colorado, $1980-2007$.

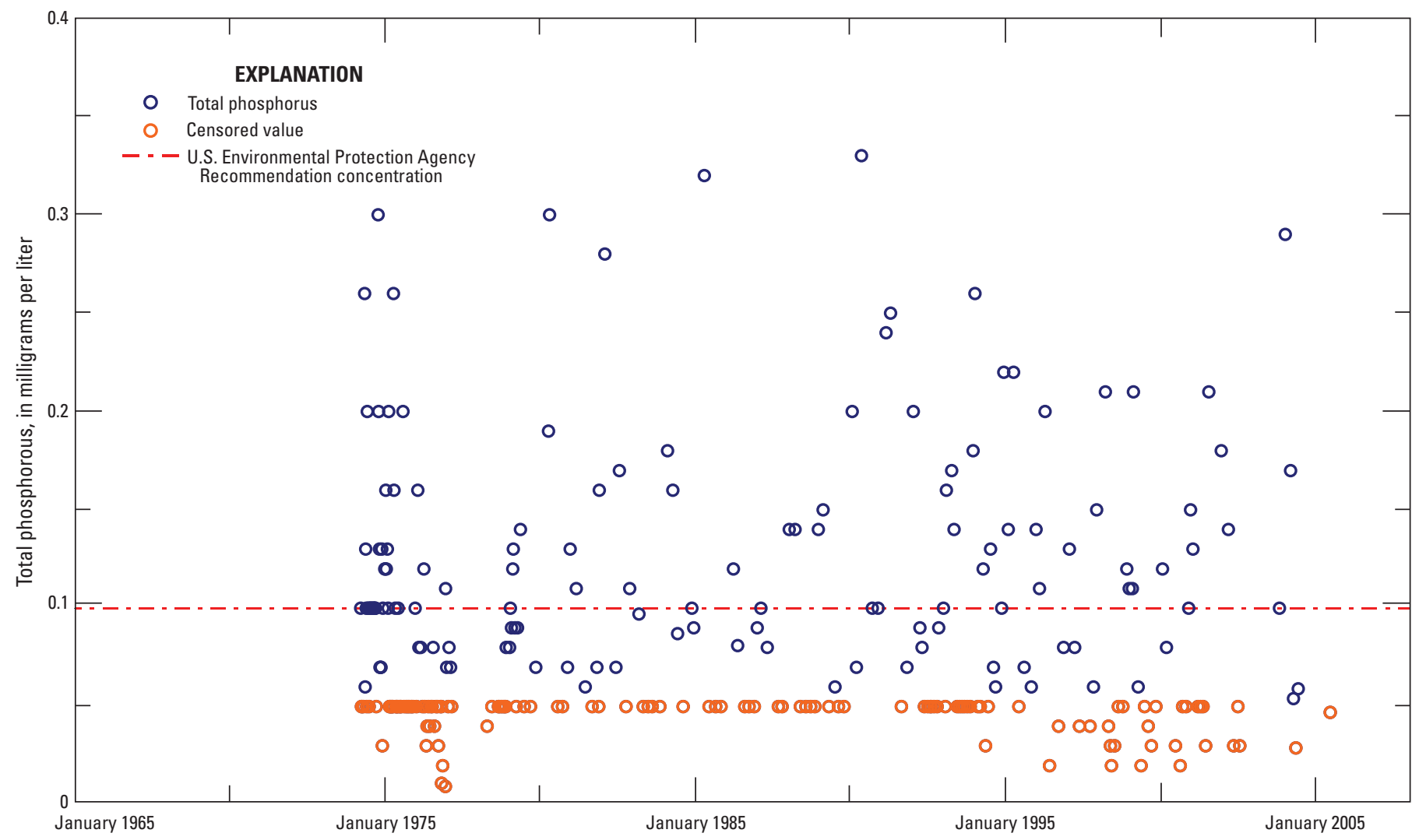

Figure 60. Temporal distribution of total phosphorus concentrations at site 227, Eagle River watershed, Colorado, 1974-2007. 
Table 18. Summary of monotonic temporal trends for nutrients in the Eagle River watershed.

[No., number; --, undetermined; mg/L, milligrams per liter]

\begin{tabular}{|c|c|c|c|c|c|c|c|c|}
\hline Site no. & $\begin{array}{l}\text { Trend } \\
\text { period }\end{array}$ & $\begin{array}{c}\text { No. of } \\
\text { samples }\end{array}$ & $\begin{array}{c}\text { Flow } \\
\text { adjustment }\end{array}$ & $\begin{array}{c}\text { Trend slope, } \\
\text { units per year }\end{array}$ & $\begin{array}{l}\text { Percent change, } \\
\text { percent per year }\end{array}$ & p-value & $\begin{array}{c}\text { Trend } \\
\text { direction }\end{array}$ & $\begin{array}{c}\text { Median } \\
\text { value }\end{array}$ \\
\hline 160 & 1996-2007 & 29 & No & -- & -- & 0.31 & No trend & 0.657 \\
\hline 173 & $1996-2007$ & 22 & No & -- & -- & .17 & No trend & .282 \\
\hline 173 & 1996-2007 & 21 & Yes & -- & -- & .82 & No trend & .282 \\
\hline 224 & 1994-2007 & 31 & No & -- & -- & .26 & No trend & .585 \\
\hline 160 & 1999-2007 & 24 & No & -- & -- & .15 & Upward & .004 \\
\hline 173 & 1999-2007 & 23 & No & -- & -- & .22 & No trend & .001 \\
\hline 224 & 1999-2007 & 24 & No & -- & -- & .51 & No trend & .009 \\
\hline \multicolumn{9}{|c|}{ Ammonia, mg/L } \\
\hline 160 & 1996-2007 & 33 & No & -- & -- & .64 & No trend & .008 \\
\hline 187B & 1996-2007 & 30 & No & -- & -- & .85 & No trend & .100 \\
\hline \multicolumn{9}{|c|}{ Total phosphorous, $\mathrm{mg} / \mathrm{L}$} \\
\hline 173 & $1996-2007$ & 29 & No & -- & -- & .80 & No trend & .037 \\
\hline 224 & $1995-2007$ & 36 & No & .0073 & 8.4 & .021 & Upward & .088 \\
\hline \multicolumn{9}{|c|}{ Orthophosphate, mg/L } \\
\hline 160 & 1996-2007 & 33 & No & -- & -- & .56 & No trend & .080 \\
\hline 173 & $1996-2007$ & 32 & No & -- & -- & .59 & No trend & .011 \\
\hline 224 & 1996-2007 & 33 & No & -- & -- & .056 & Upward & .031 \\
\hline
\end{tabular}




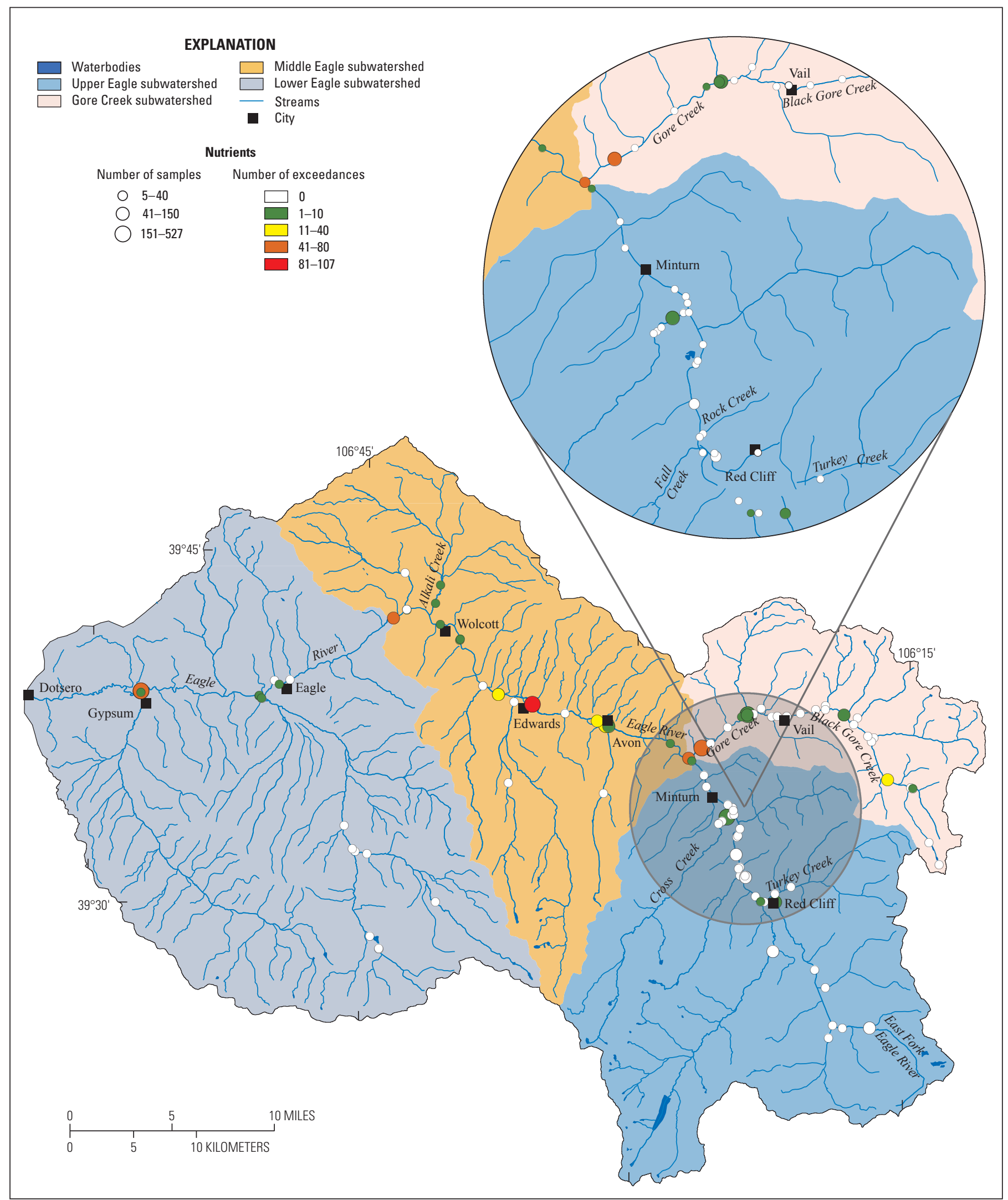

Base from U.S. Geological Survey digital data, 2007,

Universal Transverse Mercator projection

Zone 13

Figure 61. Number of exceedances of the Colorado chronic water-quality standard for nutrients at selected sites in the Eagle River watershed, Colorado, 1970-2007. 


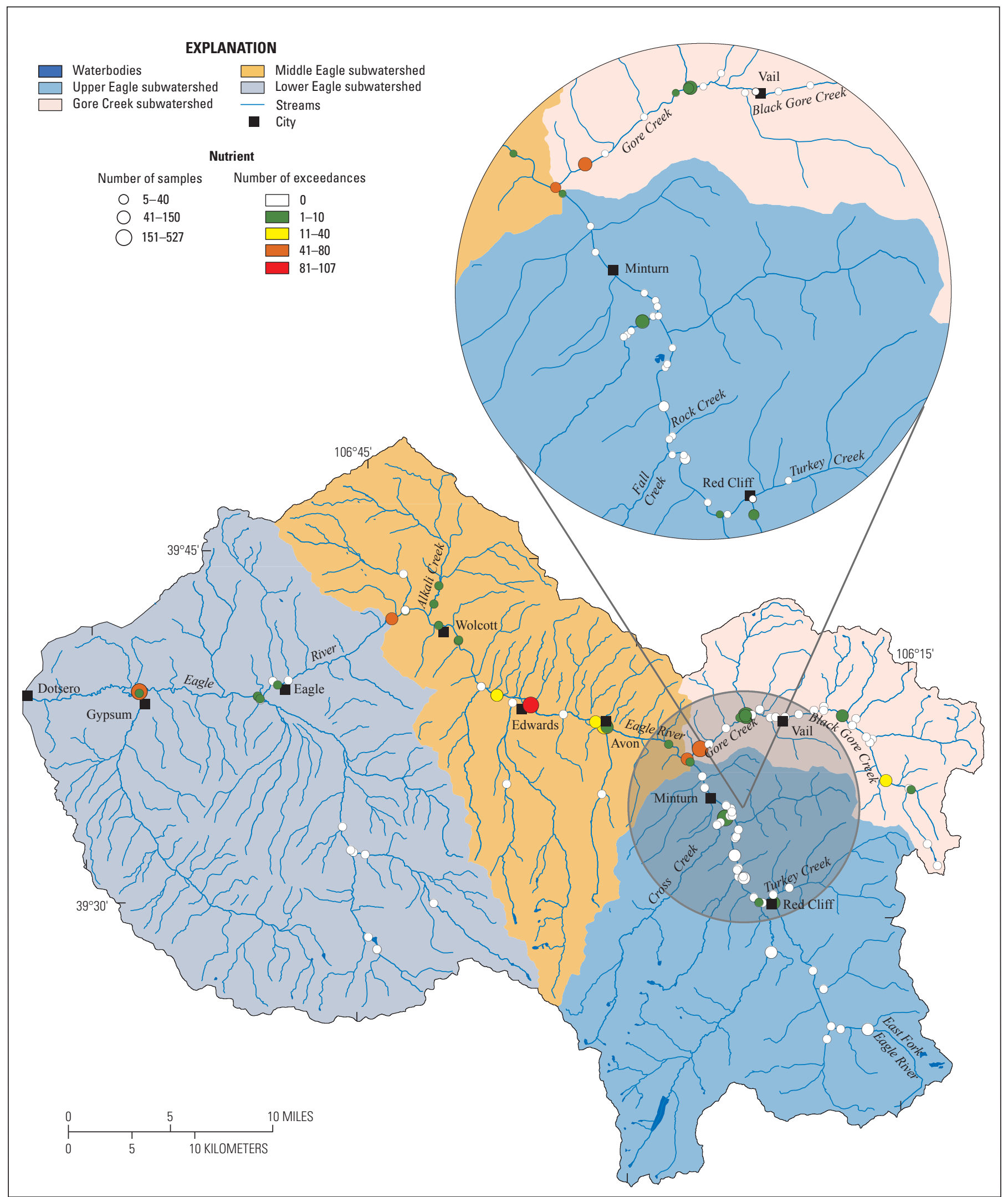

Base from U.S. Geological Survey digital data, 2007,

Universal Transverse Mercator projection

Zone 13

Figure 62. Number of exceedances of the Colorado acute water-quality standard for nutrients at selected sites in the Eagle River watershed, Colorado, 1947-2007. 
a weakly significant upward trend (p-value of 0.15 ) in nitrite at site 160 (Gore Creek subwatershed). This trend is localized to contributing areas upstream from the mouth of Gore Creek (fig. 47). No trends were detected at sites 173 (Middle Eagle subwatershed) or 224 (Lower Eagle subwatershed).

Evaluation of temporal trends was done for all sites with ammonia data; trend analysis was done at the 3 sites that met the criteria set in the "Data Sources, Compilation, Review, and Analysis" section of this report. Findings from the trend analysis are presented in table 18 and figure 48. Seasonal Kendall Trend tests were done for the time period 1996-2007. No significant trends in ammonia were detected at site 160 (Gore Creek subwatershed), 187B (Middle Eagle subwatershed), or 224 (Lower Eagle subwatershed) (fig. 48).

Evaluation of temporal trends was done for all sites with total phosphorus data; trend analysis was done at the 4 sites that met the criteria set in the "Data Sources, Compilation, Review, and Analysis" section of this report. Findings from the trend analysis are presented in table 18 and figure 49. Seasonal Kendall Trend tests were done for the time periods 1996-2007 and 1995-2007 depending on the data available at each site. There was a significant upward trend (p-value of 0.021) in total phosphorus at site 224 (Lower Eagle subwatershed) for 1995-2007. This trend represents a $0.095-\mathrm{mg} / \mathrm{L}$ increase over the entire specified time period. Upstream at sites 173 (Middle Eagle subwatershed), 160 (Gore Creek subwatershed), and 3 (Upper Eagle subwatershed) there were no trends. The area between sites 224 (Lower Eagle subwatershed) and 173 (Middle Eagle subwatershed) contains several tributaries and urban areas including the towns of Gypsum, Eagle, Edwards, and Avon (fig. 49), which may contribute to the observed trend.

Evaluation of temporal trends was done for all sites with orthophosphate data; trend analysis was done at the 3 sites that met the criteria set in the "Data Sources, Compilation, Review, and Analysis" section of this report. Findings from the trend analysis are presented in table 18 and figure 50 . The orthophosphate trend at site 224 was weakly significant (p-value of 0.056). Upstream at sites 173 (Middle Eagle subwatershed) and 160 (Gore Creek subwatershed) there were no trends in orthophosphate. The area between sites 224 (Lower Eagle subwatershed) and 173 (Middle Eagle subwatershed) contains several tributaries and urban areas including the towns of Gypsum, Eagle, Wolcott, and Edwards (fig. 50).

Three surface-water quality monitoring sites (site 140, Gore Creek below Vail wastewater treatment plant outfall at Vail; site 176, Eagle River below wastewater treatment plant at Avon; and site 187B, Eagle River $50 \mathrm{ft}$ downstream Squaw wastewater treatment plant) were sampled directly downstream from wastewater treatment plant outfalls. The streams at these sampling locations are likely not well mixed and corresponding data at these sites could potentially bias the exceedances and trend analysis. As a result these data may have increased the level and number of exceedances and affected the trend values reported.

Comparisons of nutrient concentrations to suspendedsediment concentrations were done in the ERW. A positive correlation was observed between concentrations of

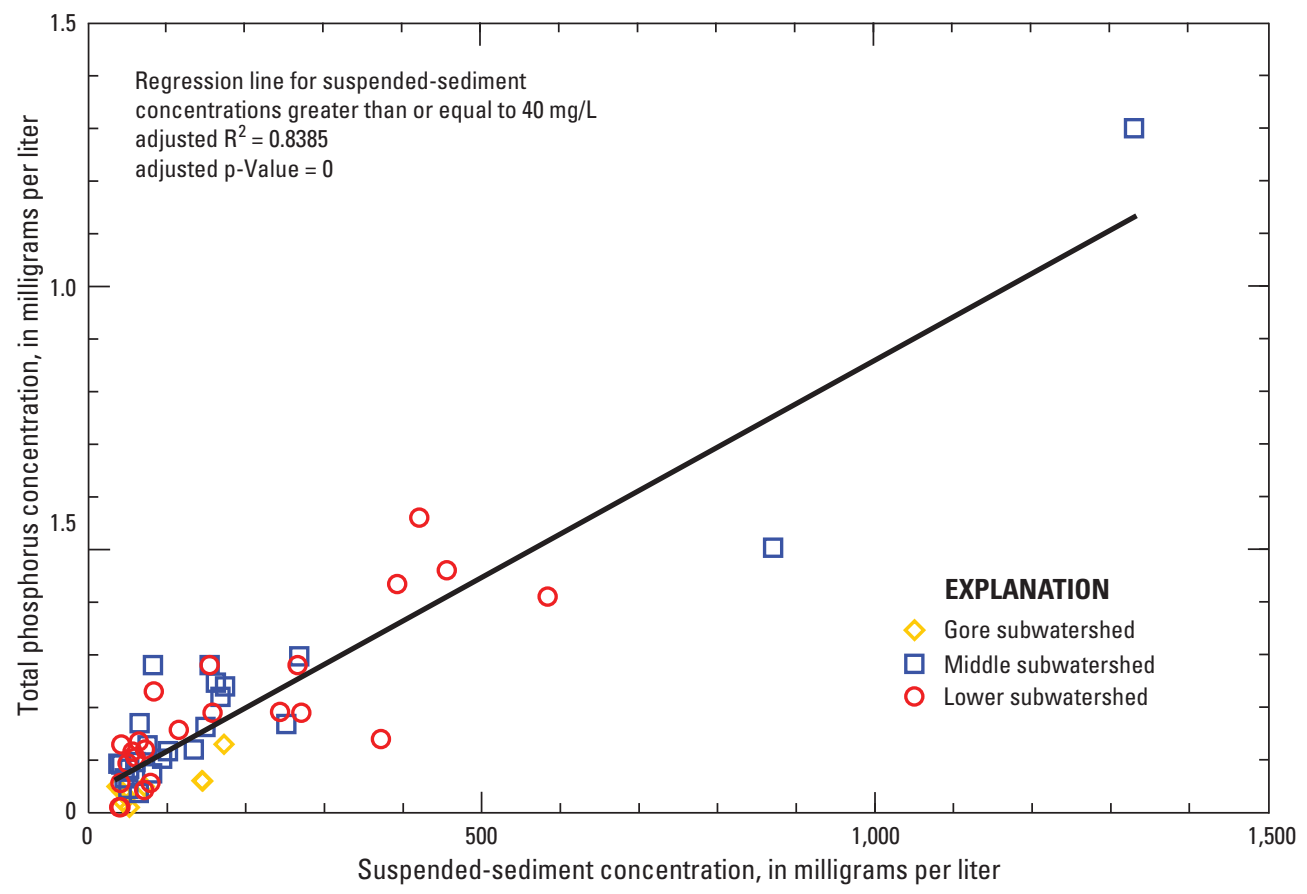

Figure 63. Correlation between suspended-sediment concentration and total phosphorus concentration in the Eagle River watershed, Colorado. 
suspended sediment greater than $40 \mathrm{mg} / \mathrm{L}$ and total phosphorus (fig. 63). Seasonal variations in total phosphorus observed within the ERW may be related to sediment transported by snowmelt runoff (February-May) and rainfall (JulySeptember) with increases in sediment possibly resulting in increases in total phosphorus concentration. No such similar correlations were detected for nitrate, nitrite, ammonia, and orthophosphate.

\section{Trace Elements}

Many trace elements in natural waters are essential to humans, plants, and aquatic life. However, trace elements can be toxic in sufficient concentrations. Trace elements often are defined as those elements that generally occur in concentrations less than 1,000 micrograms per liter (Hem, 1989). There are natural (geology) and human sources of trace elements in streams. Human sources can increase trace-element contributions to streams and can include mining, urban runoff, and municipal and industrial wastewaters. Mining (both active and historical) in the ERW has provided conduits for water and air to come in contact with the underlying geologic material, where both physical and chemical weathering can dissolve and transport these constituents into streams. Streams draining areas affected by human activities in urban areas also can mobilize trace elements.

The discussion of trace elements has been separated into two groups. Arsenic, selenium, and uranium are discussed separately from several commonly occurring trace metals (aluminum, cadmium, copper, iron, manganese, and zinc) in the following discussion.

Trace elements concentrations (arsenic, selenium, and uranium) in the ERW have been measured at 72 sites from June 8, 1966, through September 30, 2007. Concentrations for dissolved arsenic ranged from less than 1.0 to $6.0 \mu \mathrm{g} / \mathrm{L}$. The 25th, 50th, and 75th percentiles for the ERW were 0.2, 0.2 , and $5.0 \mu \mathrm{g} / \mathrm{L}$, respectively. Median concentration for the Upper Eagle subwatershed was $5.0 \mu \mathrm{g} / \mathrm{L}$ (table 19).

Concentrations for dissolved selenium ranged from less than 1.0 to $10.0 \mu \mathrm{g} / \mathrm{L}$. The $25 \mathrm{th}, 50 \mathrm{th}$, and 75 th percentiles for the ERW were $0.2,0.3$, and $0.6 \mu \mathrm{g} / \mathrm{L}$, respectively. Median concentrations for the subwatersheds were $0.2 \mu \mathrm{g} / \mathrm{L}$ in the Upper Eagle, $0.4 \mu \mathrm{g} / \mathrm{L}$ in the Middle Eagle, and $0.6 \mu \mathrm{g} / \mathrm{L}$ in the Lower Eagle (table 19).

Concentrations for dissolved uranium ranged from less than 1.0 to $9.0 \mu \mathrm{g} / \mathrm{L}$. The 25th, 50th, and 75 th percentiles for the ERW were all less than $1.0 \mu \mathrm{g} / \mathrm{L}$. Median concentrations for the subwatersheds ranged from $1.0 \mu \mathrm{g} / \mathrm{L}$ in Gore Creek, $2.0 \mu \mathrm{g} / \mathrm{L}$ in the Upper Eagle, $2.0 \mu \mathrm{g} / \mathrm{L}$ in the Middle Eagle, and $2.0 \mu \mathrm{g} / \mathrm{L}$ in the Lower Eagle (table 19).

Concentrations of trace elements in the ERW usually are below the reporting limit, and no prevailing spatial pattern was observed in the data. Areas of the Upper Eagle subwatershed and Lower Eagle subwatershed have increased concentrations relative to other areas in the ERW, and these concentrations likely are related to source geology.
Trace metal concentrations (aluminum, cadmium, iron, manganese, and zinc) in the ERW have been measured at 154 sites from April 1, 1947, through September 30, 2007. Concentrations for dissolved aluminum ranged from less than 50 to $266 \mu \mathrm{g} / \mathrm{L}$. The $25 \mathrm{th}, 50 \mathrm{th}$, and 75 th percentiles for the ERW were 14.0, 25.1, and $38.9 \mu \mathrm{g} / \mathrm{L}$, respectively. Median concentrations for the subwatersheds ranged from $8.0 \mu \mathrm{g} / \mathrm{L}$ in Gore Creek, $39.0 \mu \mathrm{g} / \mathrm{L}$ in the Upper Eagle, $20.7 \mu \mathrm{g} / \mathrm{L}$ in the Middle Eagle, to $20.7 \mu \mathrm{g} / \mathrm{L}$ in the Lower Eagle (table 19).

Concentrations for dissolved cadmium ranged from less than 0.3 to $2,500 \mu \mathrm{g} / \mathrm{L}$. The 25 th, 50 th, and 75 th percentiles for the ERW were $0.1,0.2$, and $0.5 \mu \mathrm{g} / \mathrm{L}$, respectively. Median concentrations for the subwatersheds ranged from $0.5 \mu \mathrm{g} / \mathrm{L}$ in the Upper Eagle to $0.1 \mu \mathrm{g} / \mathrm{L}$ in the Middle Eagle (table 19 and fig. 64).

Concentrations for dissolved copper were less than 5.0 to $150 \mu \mathrm{g} / \mathrm{L}$. The 25 th, 50 th, and 75 th percentiles for the ERW were $1,2.2$, and $5.0 \mu \mathrm{g} / \mathrm{L}$, respectively. Median concentrations for the subwatersheds were $1.4 \mathrm{mg} / \mathrm{L}$ in Gore Creek, $3.0 \mu \mathrm{g} / \mathrm{L}$ in the Upper Eagle, $2.0 \mu \mathrm{g} / \mathrm{L}$ in the Middle Eagle, to $1.9 \mu \mathrm{g} / \mathrm{L}$ in the Lower Eagle (table 19 and fig. 65).

Concentrations for dissolved iron ranged from less than 3 to $39,000 \mu \mathrm{g} / \mathrm{L}$. The 25th, 50th, and 75th percentiles for the ERW were 22, 90, and $200 \mu \mathrm{g} / \mathrm{L}$, respectively. Median concentrations for the subwatersheds were $13.0 \mu \mathrm{g} / \mathrm{L}$ in Gore Creek, $140 \mu \mathrm{g} / \mathrm{L}$ in the Upper Eagle, $40 \mu \mathrm{g} / \mathrm{L}$ in the Middle Eagle, and $30 \mu \mathrm{g} / \mathrm{L}$ in the Lower Eagle (table 19 and fig. 66).

Concentrations for dissolved manganese ranged from less than 10 to $100,000 \mu \mathrm{g} / \mathrm{L}$. The 25 th, 50th, and 75th percentiles for the ERW were 13.0, 62.0, and $310 \mu \mathrm{g} / \mathrm{L}$, respectively. Median concentrations for the subwatersheds were $6.0 \mu \mathrm{g} / \mathrm{L}$ in Gore Creek, $160 \mu \mathrm{g} / \mathrm{L}$ in the Upper Eagle, $71 \mu \mathrm{g} / \mathrm{L}$ in the Middle Eagle, and $31.7 \mu \mathrm{g} / \mathrm{L}$ in the Lower Eagle (table 19 and fig. 67).

Concentrations for dissolved zinc ranged from less than 10 to $240,000 \mu \mathrm{g} / \mathrm{L}$. The $25 \mathrm{th}, 50 \mathrm{th}$, and 75 th percentiles for the ERW were 10.0, 70, and $330 \mu \mathrm{g} / \mathrm{L}$, respectively. Median concentrations for the subwatersheds were $5.7 \mu \mathrm{g} / \mathrm{L}$ in Gore Creek, $180 \mu \mathrm{g} / \mathrm{L}$ in the Upper Eagle, $50 \mu \mathrm{g} / \mathrm{L}$ in the Middle Eagle, and $22.2 \mu \mathrm{g} / \mathrm{L}$ in the Lower Eagle (table 19 and fig. 68).

The spatial pattern shows an increase in dissolved trace-metals concentrations as water moves downstream from historic mining areas (fig. 5), with a defined increase in the Eagle River and several tributaries near Belden (figs. 64-68). Concentrations often decreased or are below reporting limits in Gore Creek and other tributaries within the ERW. Seasonal variations generally show that many trace metals concentrations tend to be lowest May-June and highest NovemberApril. A gradual change in concentrations between seasons may be related to dilution effects from streamflow. A notable exception to this pattern occurs in early spring (FebruaryApril) along the leading edge of snowmelt (figs. 69-73). Increases in melt waters moving along intermittent flow paths interact with mine shafts and (or) adits, mineralized rock units, and other areas affected by processed mine wastes and 
Table 19. Summary statistics of trace element data in the Eagle River watershed by subwatershed 1947-2007.

[No., number; MRL, Minimum Reporting Limit; <, less than; --, undetermined]

\begin{tabular}{|c|c|c|c|c|c|c|c|c|}
\hline \multirow{2}{*}{$\begin{array}{l}\text { Constituent } \\
\text { or property }\end{array}$} & \multirow{2}{*}{$\begin{array}{l}\text { No. of } \\
\text { censored } \\
\text { values }\end{array}$} & \multirow{2}{*}{$\begin{array}{c}\text { No. of } \\
\text { samples }\end{array}$} & \multirow[t]{2}{*}{ MRL } & \multirow[t]{2}{*}{ Minimum ${ }^{1}$} & \multicolumn{3}{|c|}{$\begin{array}{l}\text { Concentration or value } \\
\text { at indicated percentile }\end{array}$} & \multirow[t]{2}{*}{ Maximum } \\
\hline & & & & & 25 & 50 & 75 & \\
\hline \multicolumn{9}{|c|}{ Gore Creek subwatershed } \\
\hline Aluminum, dissolved $(\mu \mathrm{g} / \mathrm{L})$ & 19 & 40 & 50 & 2.0 & 5.0 & 8.0 & 12.0 & 50 \\
\hline Aluminum, total $(\mu \mathrm{g} / \mathrm{L})$ & 2 & 22 & 15.0 & 15.0 & 28.0 & 65 & 195 & 1,288 \\
\hline Arsenic, dissolved $(\mu \mathrm{g} / \mathrm{L})$ & 66 & 66 & 1.0 & 1.0 & -- & -- & -- & $<1.0$ \\
\hline Arsenic, total $(\mu \mathrm{g} / \mathrm{L})$ & 101 & 103 & 1.0 & 1.0 & -- & -- & -- & 8.0 \\
\hline Barium, dissolved $(\mu \mathrm{g} / \mathrm{L})$ & 0 & 21 & -- & 18.0 & 58 & 118 & 127 & 190 \\
\hline Barium, total $(\mu \mathrm{g} / \mathrm{L})$ & 0 & 0 & -- & -- & -- & -- & -- & -- \\
\hline Cadmium, dissolved $(\mu \mathrm{g} / \mathrm{L})$ & 201 & 219 & .3 & .10 & -- & -- & -- & 1.2 \\
\hline Cadmium, total $(\mu \mathrm{g} / \mathrm{L})$ & 88 & 91 & 1.0 & .10 & -- & -- & -- & $<1.0$ \\
\hline Chromium, dissolved $(\mu \mathrm{g} / \mathrm{L})$ & 30 & 35 & 1.0 & 1.0 & -- & -- & -- & 23.0 \\
\hline Chromium, total $(\mu \mathrm{g} / \mathrm{L})$ & 12 & 12 & 1.0 & 1.0 & -- & -- & -- & $<1.0$ \\
\hline Copper, dissolved ( $\mu \mathrm{g} / \mathrm{L})$ & 218 & 304 & 5.0 & .4 & .9 & 1.4 & 2.4 & 60 \\
\hline Copper, total $(\mu \mathrm{g} / \mathrm{L})$ & 50 & 91 & 1.0 & 1.0 & .3 & 0.8 & 1.4 & 47 \\
\hline Iron, dissolved $(\mu \mathrm{g} / \mathrm{L})$ & 154 & 393 & 20.0 & 3.0 & 8.0 & 13.0 & 20.0 & 190 \\
\hline Iron, total $(\mu \mathrm{g} / \mathrm{L})$ & 51 & 338 & 20.0 & 18.0 & 24.0 & 70 & 190 & 1,900 \\
\hline Lead, dissolved $(\mu \mathrm{g} / \mathrm{L})$ & 230 & 247 & 5.0 & .04 & -- & -- & -- & 14.0 \\
\hline Lead, total $(\mu \mathrm{g} / \mathrm{L})$ & 88 & 91 & 3.0 & .8 & -- & -- & -- & 4.2 \\
\hline Manganese, dissolved $(\mu \mathrm{g} / \mathrm{L})$ & 189 & 525 & 10.0 & .8 & 2.4 & 6.0 & 10.0 & 530 \\
\hline Manganese, total $(\mu \mathrm{g} / \mathrm{L})$ & 73 & 324 & 10.0 & 1.6 & 6.7 & 10.0 & 21.0 & 320 \\
\hline Mercury, dissolved $(\mu \mathrm{g} / \mathrm{L})$ & 15 & 15 & .1 & .1 & -- & -- & -- & $<0.1$ \\
\hline Mercury, total $(\mu \mathrm{g} / \mathrm{L})$ & 72 & 73 & .2 & .2 & -- & -- & -- & 0.3 \\
\hline Molybdenum, dissolved $(\mu \mathrm{g} / \mathrm{L})$ & 20 & 21 & 1.0 & 1.0 & -- & -- & -- & 4.0 \\
\hline Molybdenum, total $(\mu \mathrm{g} / \mathrm{L})$ & 0 & 0 & -- & -- & -- & -- & -- & -- \\
\hline Nickel, dissolved $(\mu \mathrm{g} / \mathrm{L})$ & 24 & 35 & 1.0 & .04 & .01 & .2 & .7 & 26.0 \\
\hline Nickel, total $(\mu \mathrm{g} / \mathrm{L})$ & 12 & 12 & 1.0 & 1.0 & -- & -- & -- & $<1.0$ \\
\hline Selenium, dissolved $(\mu \mathrm{g} / \mathrm{L})$ & 56 & 70 & 1.0 & .08 & -- & -- & -- & 4.4 \\
\hline Selenium, total $(\mu \mathrm{g} / \mathrm{L})$ & 87 & 98 & 2.0 & 1.0 & -- & -- & -- & 5.0 \\
\hline Silver, dissolved $(\mu \mathrm{g} / \mathrm{L})$ & 133 & 139 & .2 & .1 & -- & -- & -- & .3 \\
\hline Silver, total $(\mu \mathrm{g} / \mathrm{L})$ & 51 & 51 & .2 & .2 & -- & -- & -- & $<.2$ \\
\hline Uranium, dissolved $(\mu \mathrm{g} / \mathrm{L})$ & 13 & 37 & 1.0 & 1.0 & -- & 1.0 & 2.0 & 4.1 \\
\hline Zinc, dissolved $(\mu \mathrm{g} / \mathrm{L})$ & 257 & 425 & 10.0 & .6 & 3.2 & 5.7 & 10.0 & 149 \\
\hline Zinc, total $(\mu \mathrm{g} / \mathrm{L})$ & 158 & 202 & 10.0 & 5.0 & .9 & 2.7 & 8.1 & 370 \\
\hline \multicolumn{9}{|c|}{ Upper Eagle subwatershed } \\
\hline Aluminum, dissolved $(\mu \mathrm{g} / \mathrm{L})$ & 34 & 86 & 50 & 10.0 & 24.0 & 39.0 & 54 & 180 \\
\hline Aluminum, total $(\mu \mathrm{g} / \mathrm{L})$ & 0 & 17 & -- & 90 & 106 & 140 & 153 & 189 \\
\hline Arsenic, dissolved $(\mu \mathrm{g} / \mathrm{L})$ & 75 & 338 & 1.0 & .1 & .2 & 5.0 & 5.0 & 6.0 \\
\hline Arsenic, total $(\mu \mathrm{g} / \mathrm{L})$ & 66 & 69 & 1.0 & -- & -- & -- & -- & -- \\
\hline Barium, dissolved $(\mu \mathrm{g} / \mathrm{L})$ & 0 & 14 & -- & 12.0 & 30.0 & 41.5 & 51 & 100 \\
\hline Cadmium, dissolved $(\mu \mathrm{g} / \mathrm{L})$ & 281 & 1,805 & .3 & .02 & .2 & .5 & 1.0 & 2,500 \\
\hline Cadmium, total $(\mu \mathrm{g} / \mathrm{L})$ & 33 & 131 & 1.0 & .14 & .4 & .7 & 1.3 & 4.56 \\
\hline Chromium, dissolved $(\mu \mathrm{g} / \mathrm{L})$ & 42 & 47 & 1.0 & -- & --- & -- & -- & -- \\
\hline Chromium, total $(\mu \mathrm{g} / \mathrm{L})$ & 0 & 0 & -- & -- & -- & -- & -- & -- \\
\hline Copper, dissolved $(\mu \mathrm{g} / \mathrm{L})$ & 268 & 1,828 & 5.0 & .2 & 1.0 & 3.0 & 5.0 & 150 \\
\hline Copper, total $(\mu \mathrm{g} / \mathrm{L})$ & 100 & 250 & 1.0 & 1.0 & 1.0 & 3.0 & 12.9 & 1,150 \\
\hline Iron, dissolved $(\mu \mathrm{g} / \mathrm{L})$ & 62 & 1,636 & 20.0 & 10.0 & 80 & 140 & 270 & 39,000 \\
\hline Iron, total $(\mu \mathrm{g} / \mathrm{L})$ & 9 & 903 & 20.0 & 20.0 & 300 & 600 & 1,510 & $1,000,000$ \\
\hline Lead, dissolved $(\mu \mathrm{g} / \mathrm{L})$ & 527 & 1,780 & 5.0 & .04 & 1.0 & 1.0 & 3.0 & 40 \\
\hline Lead, total $(\mu \mathrm{g} / \mathrm{L})$ & 207 & 242 & 3.0 & -- & -- & -- & -- & -- \\
\hline Manganese, dissolved $(\mu \mathrm{g} / \mathrm{L})$ & 106 & 1,926 & 10.0 & 1.5 & 30.0 & 160 & 605 & 100,000 \\
\hline Manganese, total $(\mu \mathrm{g} / \mathrm{L})$ & 31 & 414 & 10.0 & 3.3 & 21.7 & 325 & 1,600 & 156,000 \\
\hline Mercury, dissolved $(\mu \mathrm{g} / \mathrm{L})$ & 41 & 42 & .1 & -- & -- & -- & -- & -- \\
\hline Mercury, total $(\mu \mathrm{g} / \mathrm{L})$ & 28 & 28 & .2 & -- & -- & -- & -- & -- \\
\hline Molybdenum, dissolved $(\mu \mathrm{g} / \mathrm{L})$ & 0 & 1 & 5.0 & -- & -- & -- & -- & -- \\
\hline Molybdenum, total $(\mu \mathrm{g} / \mathrm{L})$ & 1 & 1 & 50 & -- & -- & -- & -- & -- \\
\hline Nickel, dissolved $(\mu \mathrm{g} / \mathrm{L})$ & 8 & 23 & 1.0 & .1 & .5 & .8 & .9 & 30 \\
\hline Nickel, total $(\mu \mathrm{g} / \mathrm{L})$ & 0 & 0 & -- & -- & -- & -- & -- & -- \\
\hline
\end{tabular}


Table 19. Summary statistics of trace element data in the Eagle River watershed by subwatershed 1947-2007.-Continued

[No., number; MRL, Minimum Reporting Limit; <, less than; --, undetermined]

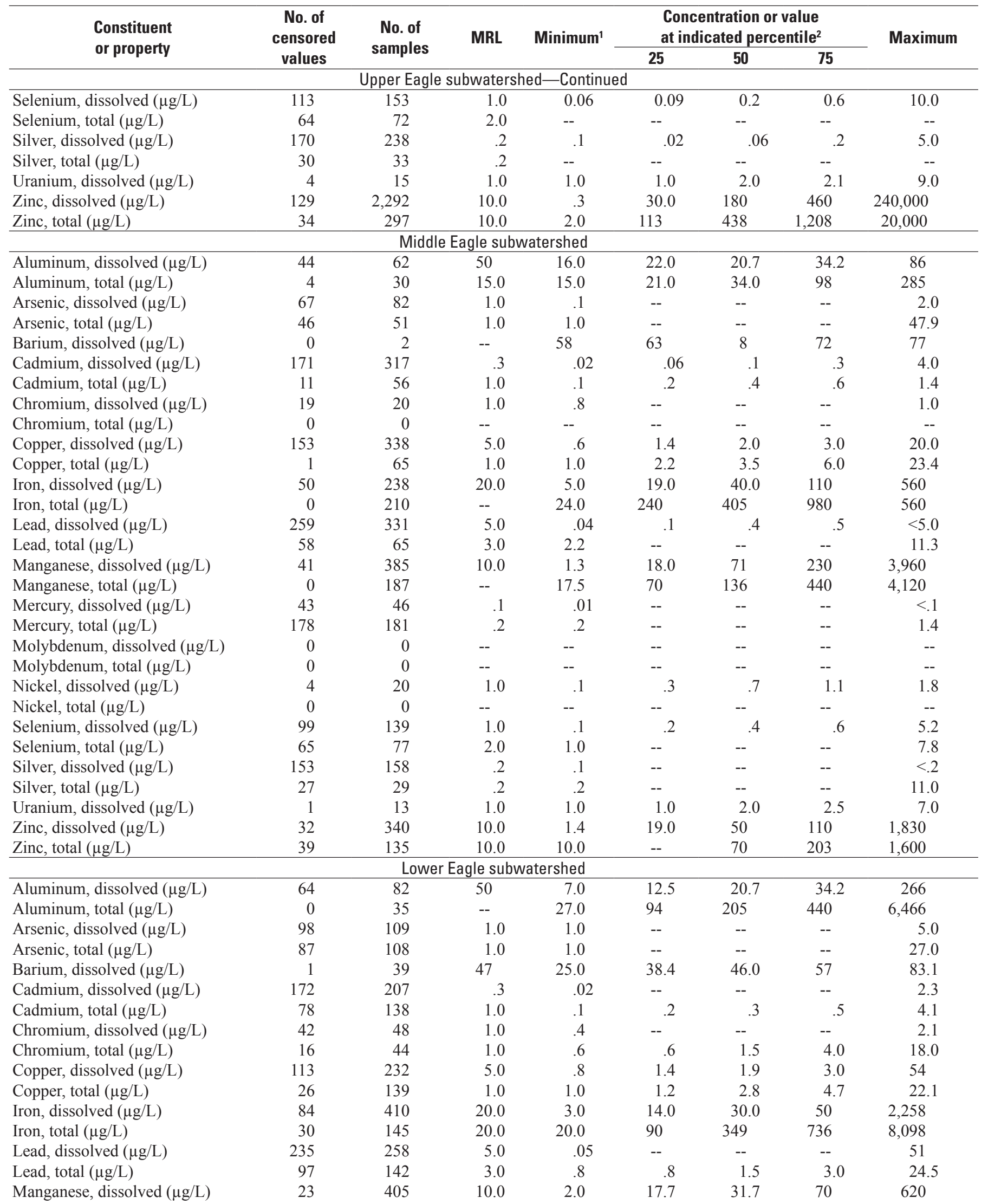


Table 19. Summary statistics of trace element data in the Eagle River watershed by subwatershed 1947-2007.-Continued

[No., number; MRL, Minimum Reporting Limit; <, less than; --, undetermined]

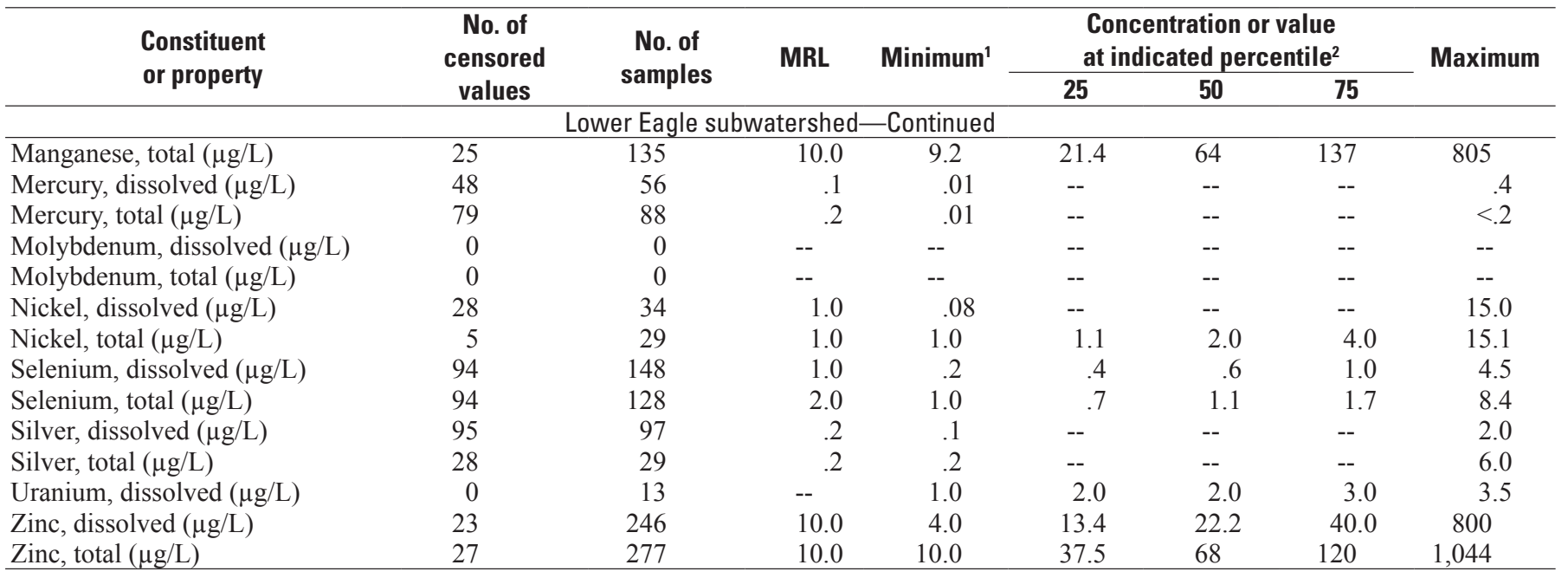

${ }^{1}$ Uncensored values.

${ }^{2}$ Percentile values can be less than the MRL values due to multiple MRLs within the combined dataset.

contaminated soils. Increases in melt water also mobilize localized areas of concentrated metals, leading to increases in some metal concentrations in streams. The increase in metal concentrations represents a seasonal "flushing" that becomes less pronounced as snowmelt increases (Colorado Department of Public Health and Environment and U.S. Environmental Protection Agency, 2000). In addition, the increase in median metal concentrations coincides with the reduction in hardness concentrations; this is illustrated for cadmium (fig. 74). Reductions in hardness can be an important consideration in the assessment of metals toxicity to aquatic biota (see "Hardness" section of this report).

The seasonal pattern of dissolved iron at site 224 (Lower Eagle subwatershed) is different from other metals and other sites within the ERW (fig. 71). At site 224, dissolved iron concentrations are relatively stable for most of the year with higher concentrations April-July. These increased concentrations are not representative of the rest of the ERW where increases in streamflow result in dilution of many constituents. The increased concentrations of dissolved iron may best be explained by the colloidal behavior of iron. Colloidal iron is suspended iron that is not dissolved, but can potentially be included in concentrations of dissolved iron because of the nature of sample-collection techniques (Stumm and Morgan, 1996). Commonly, water samples are filtered using 0.45-micron filters. Iron colloids can be less than the filterpore size, in which case not all colloids would be restricted from passing through the filter. If colloidal forms of iron are included in dissolved iron concentrations at this site, this may account for the difference in seasonal patterns at site 224 because higher concentrations of total iron also occur MarchMay at site 224 (fig. 75). The increases in iron concentrations during high flow may be the result of re-suspension of deposited iron colloids in the stream sediments during higher streamflows when increases in turbulence and stream power increase transport and suspension of bed sediments (Church and others, 1997; Schemel and others, 1999). Another possible explanation for the observed increases in dissolved iron at site 224 in April-June may be from seasonal variations in iron sources from areas downstream from site 66 (Upper Eagle subwatershed), although no specific source has been identified.

Arsenic, selenium, uranium, in addition to several commonly occurring metals (aluminum, cadmium, copper, iron, lead, manganese, mercury, nickel, silver, and zinc) in this report were compared to available State standards set by the CDPHE Regulation no. 33 (Colorado Department of Public Health and Environment, 2007). Number of exceedances is based on dissolved sample concentrations, except for iron which is based on dissolved and total recoverable concentrations.

A chronic State standard of $150 \mu \mathrm{g} / \mathrm{L}$ applies statewide for arsenic in the ERW, except for segment 11 which uses a TVS based on total recoverable concentrations $(100 \mu \mathrm{g} / \mathrm{L})$. All reaches of the ERW use the acute TVS for arsenic $(50 \mu \mathrm{g} / \mathrm{L})$ except for segment 11 (340 $\mu \mathrm{g} / \mathrm{L})$ (fig. 12). There are no exceedances of the chronic State standards for arsenic in the ERW out of 331 comparisons. There is 1 exceedance of the acute State standard at site 27 (Upper Eagle subwatershed) in 1971.

State standards for selenium are designated by the CDPHE and apply to all reaches of the ERW. The chronic State standard for dissolved selenium is $4.6 \mu \mathrm{g} / \mathrm{L}$, and the acute State standard is $18.4 \mu \mathrm{g} / \mathrm{L}$ for dissolved selenium. Out of the 510 comparisons of selenium measurements to State standards, approximately 1 percent (5 comparisons) exceeded the chronic State standard for selenium. There were no exceedances out of 510 comparisons of the acute State standard for selenium (table 20). 


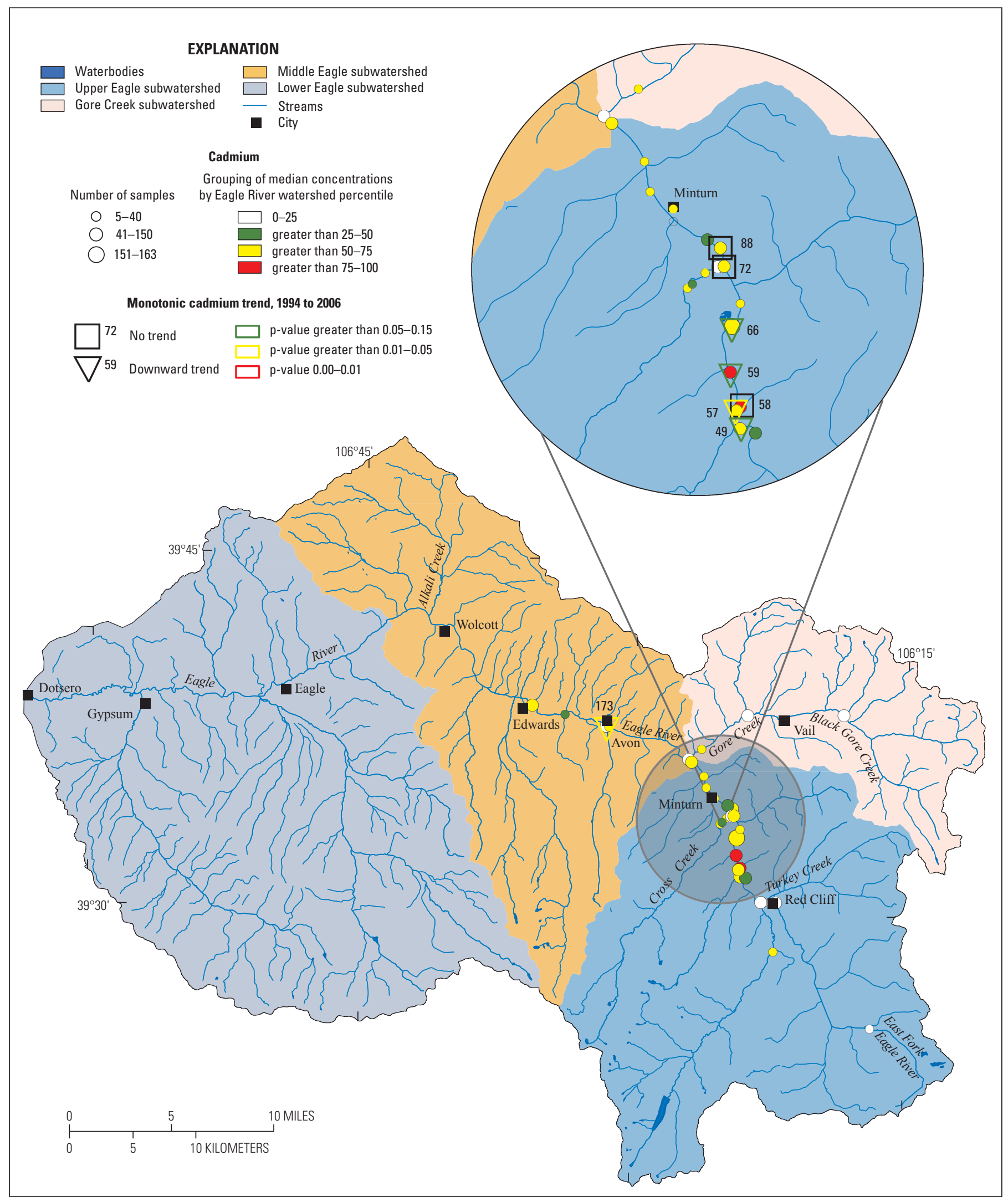

Base from U.S. Geological Survey digital data, 2007,

Universal Transverse Mercator projection

Zone 13

Figure 64. Spatial distribution of median-concentration percentiles and monotonic dissolved cadmium trends at selected sites in the Eagle River watershed, Colorado, 1994-2006. 


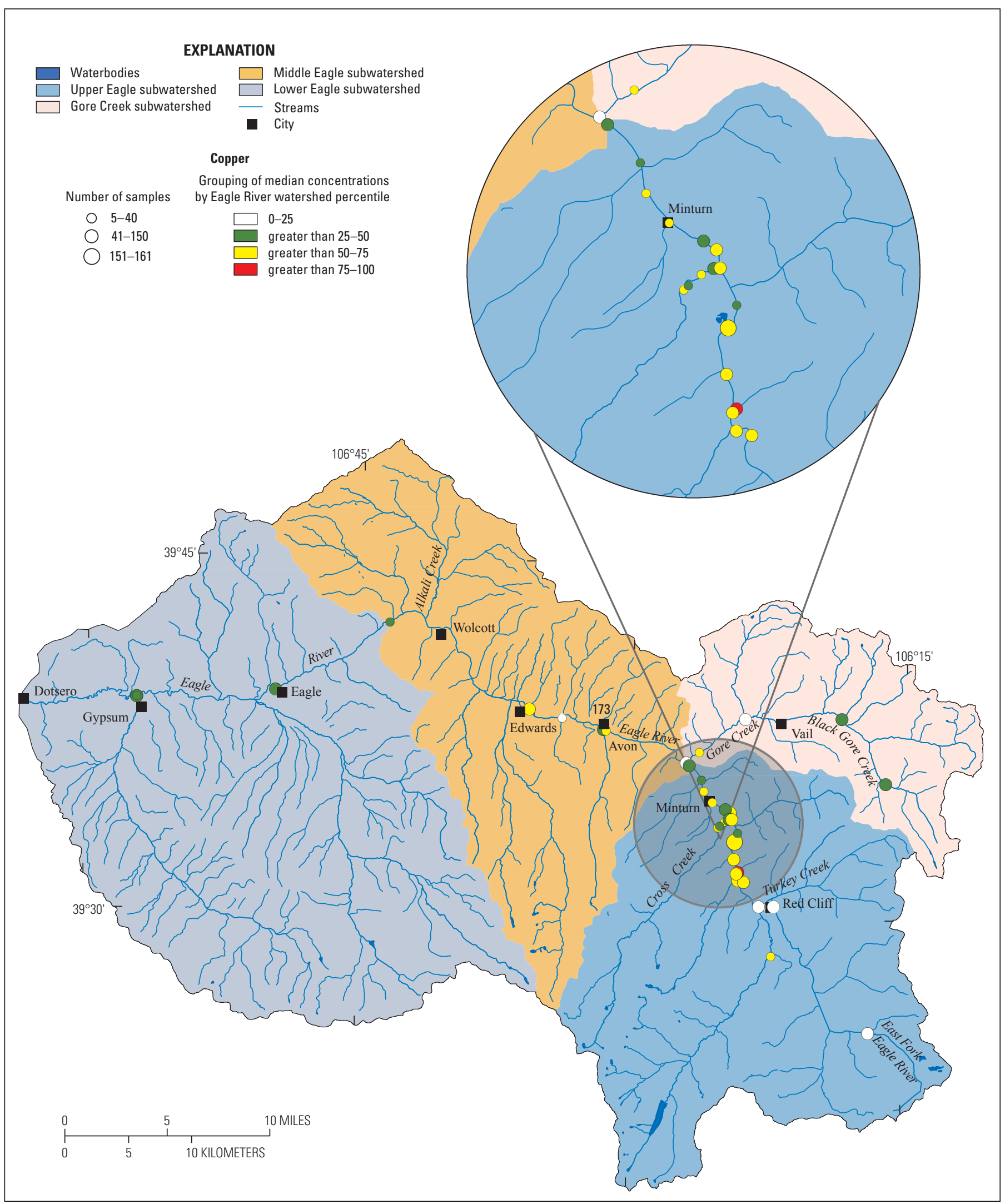

Base from U.S. Geological Survey digital data, 2007

Universal Transverse Mercator projection

Zone 13

Figure 65. Spatial distribution of median-concentration percentiles for dissolved copper at selected sites in the Eagle River watershed, Colorado, 1966-2007. 


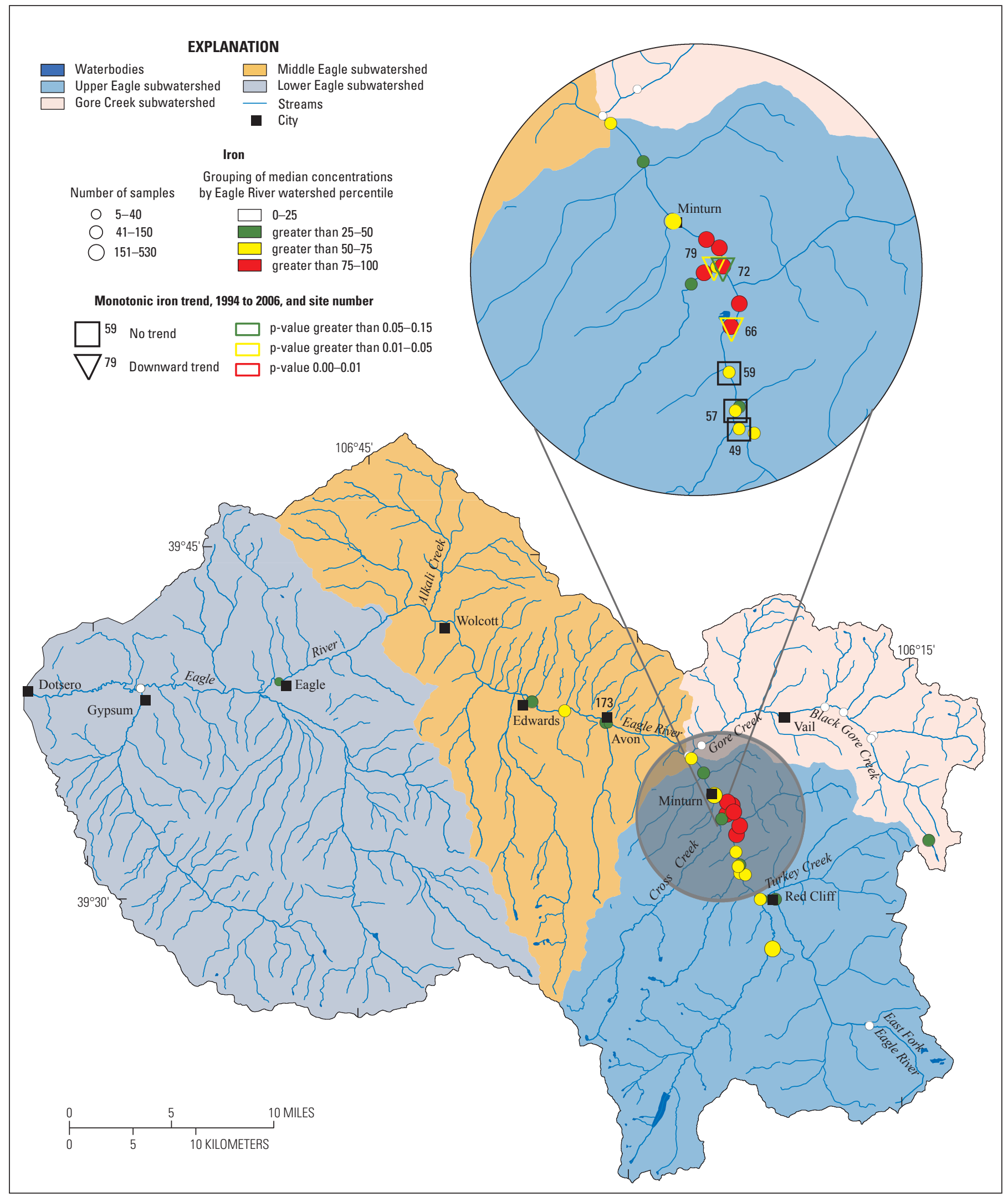

Base from U.S. Geological Survey digital data, 2007,

Universal Transverse Mercator projection

Zone 13

Figure 66. Spatial distribution of median-concentration percentiles and monotonic dissolved iron trends at selected sites in the Eagle River watershed, Colorado, 1994-2006. 


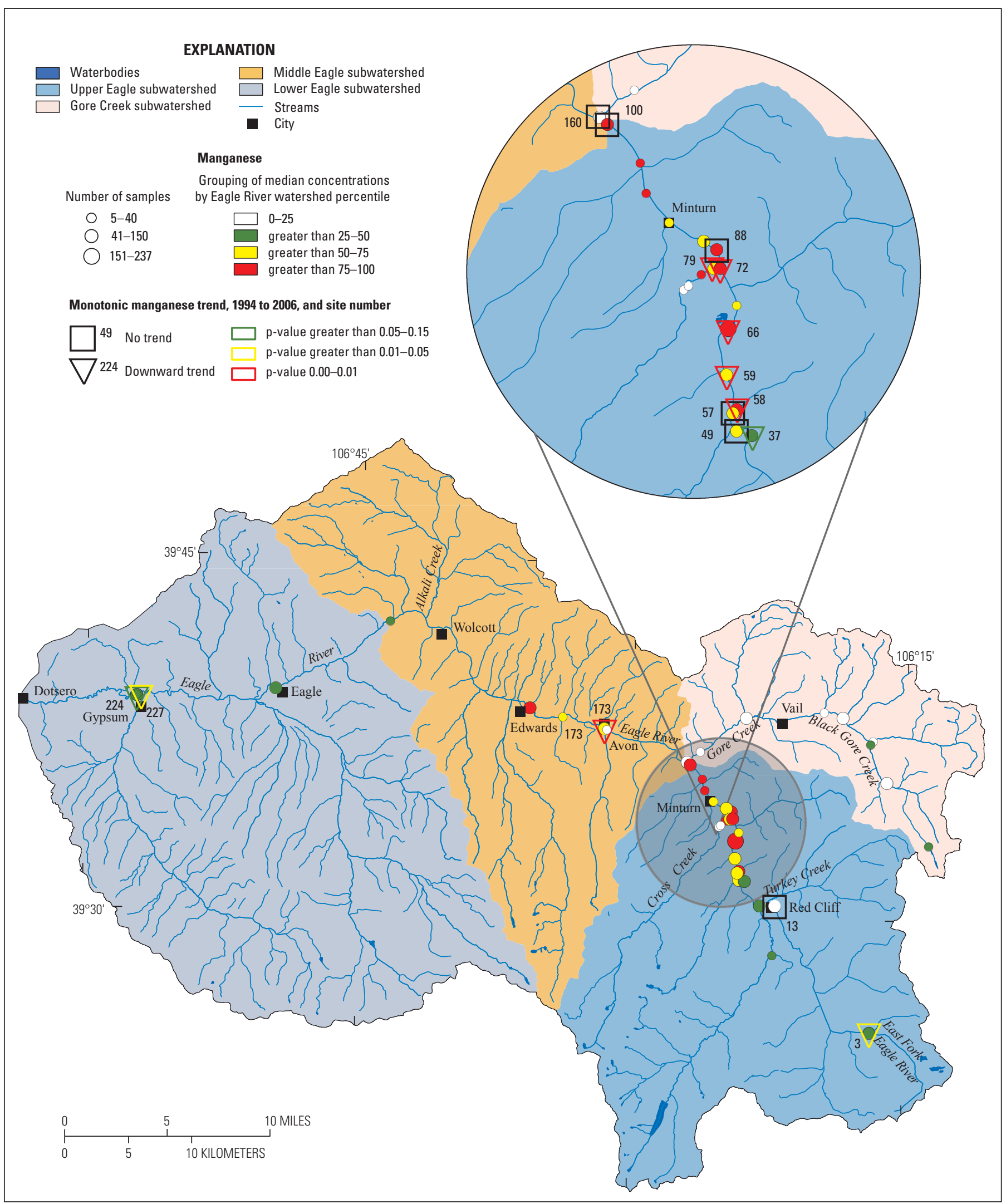

Base from U.S. Geological Survey digital data, 2007,

Universal Transverse Mercator projection

Zone 13

Figure 67. Spatial distribution of median-concentration percentiles and monotonic dissolved manganese trends at selected sites in the Eagle River watershed, Colorado, 1994-2006. 


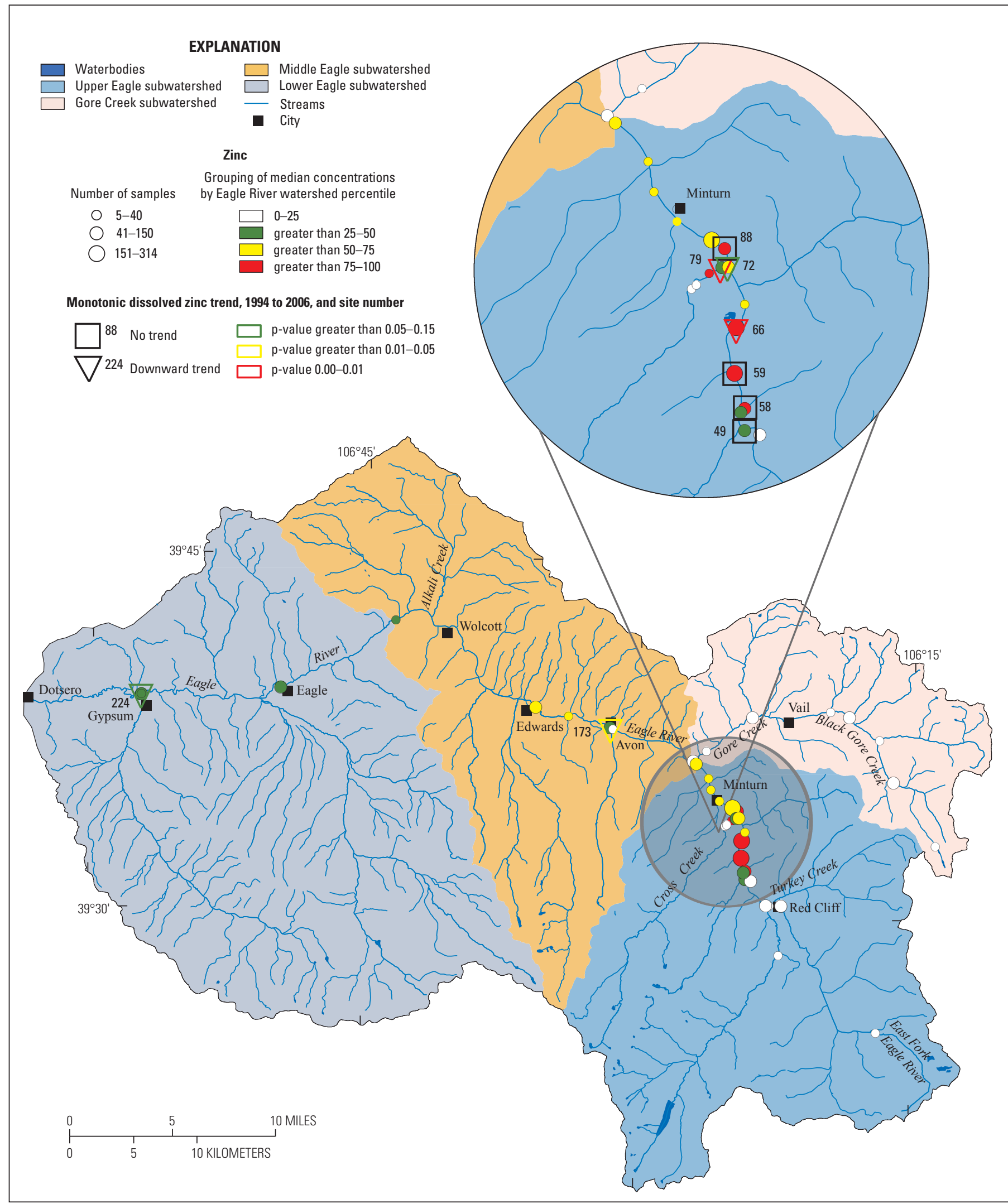

Base from U.S. Geological Survey digital data, 2007,

Universal Transverse Mercator projection

Zone 13

Figure 68. Spatial distribution of median-concentration percentiles and monotonic dissolved zinc concentration trends at selected sites in the Eagle River watershed, Colorado, 1994-2006. 


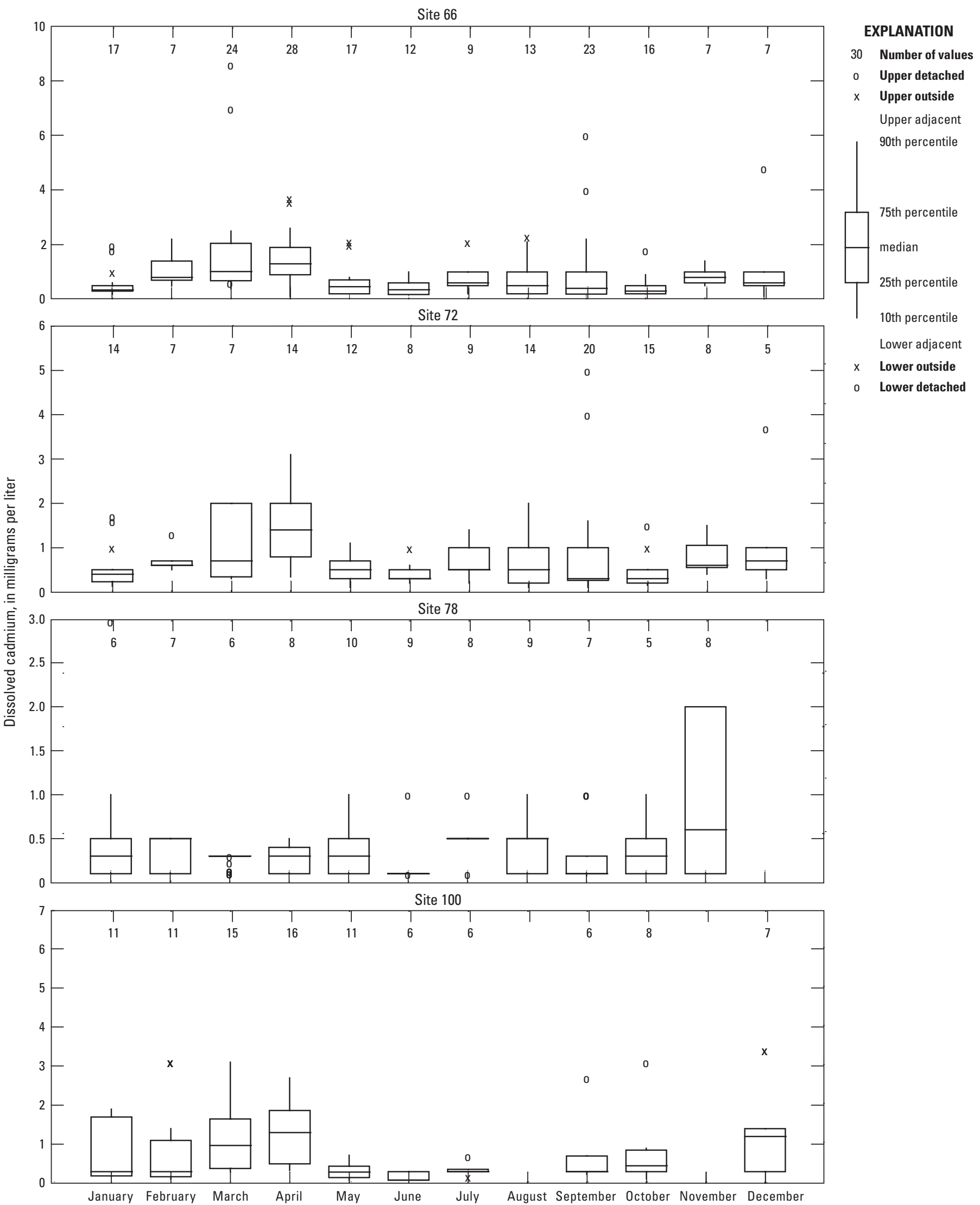

Figure 69. Seasonal pattern of dissolved cadmium concentrations at selected sites in the Eagle River watershed, Colorado, $1966-2007$. 


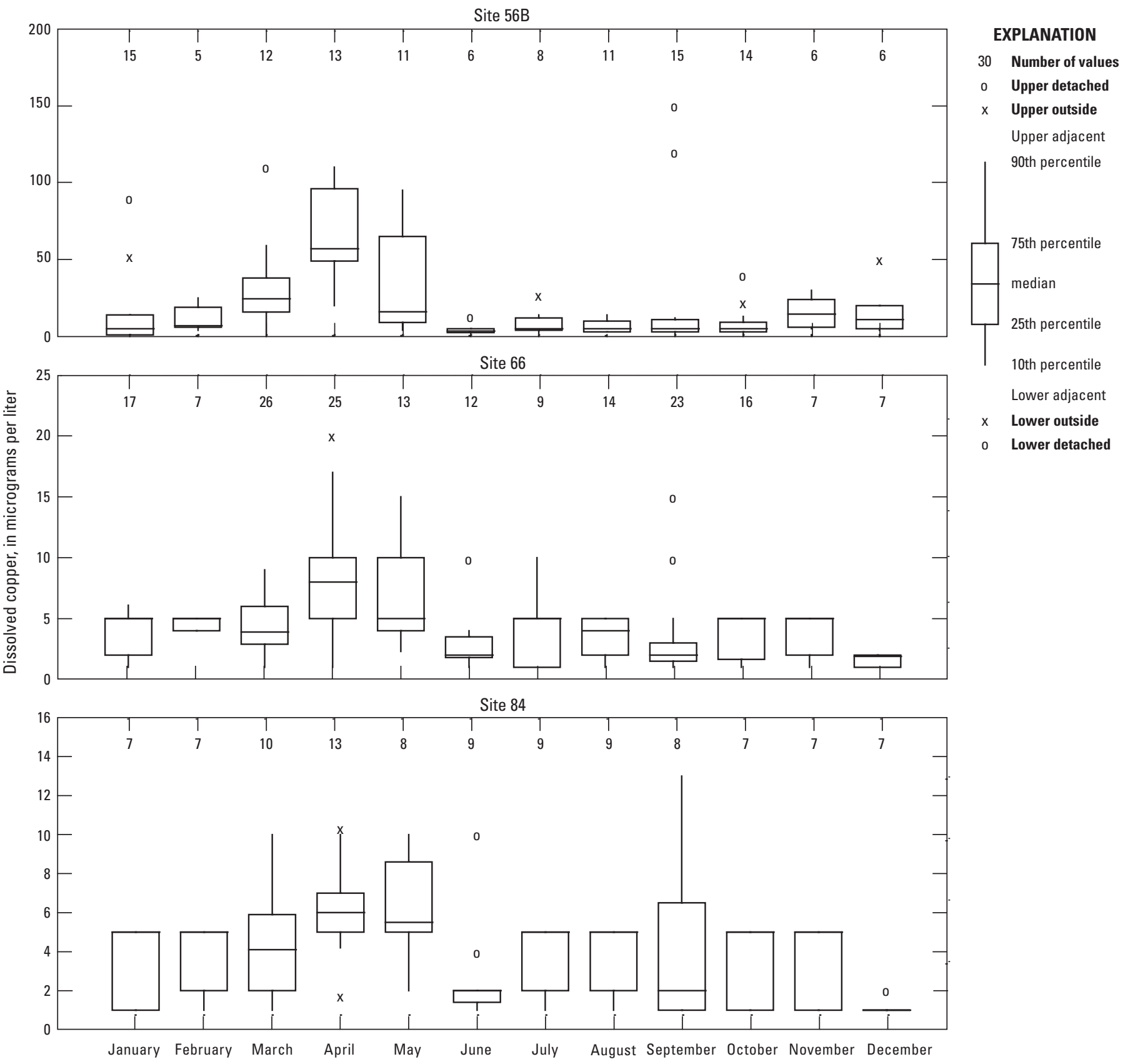

Figure 70. Seasonal pattern of dissolved copper concentrations at selected sites in the Eagle River watershed, Colorado, $1966-2007$. 


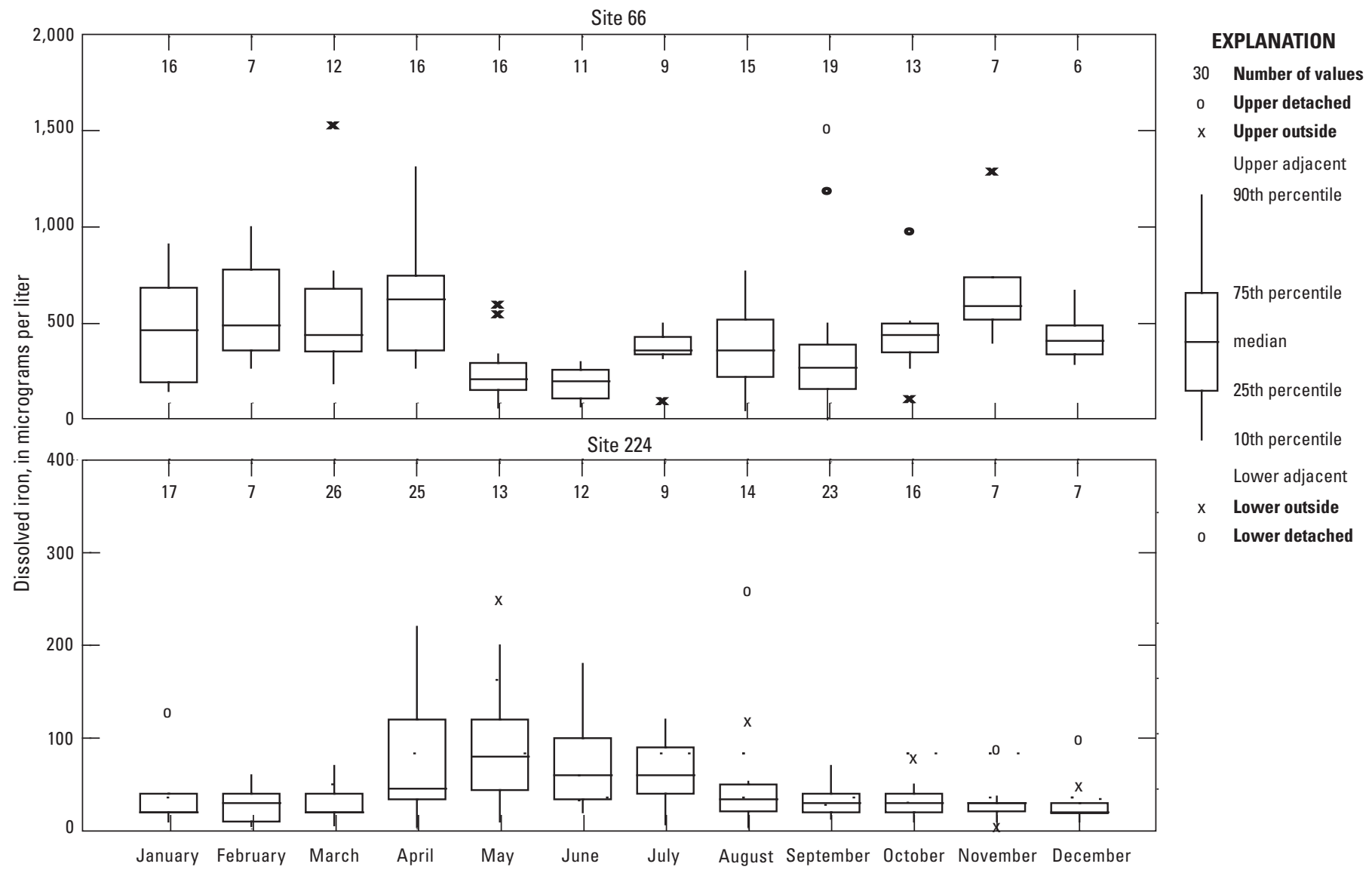

Figure 71. Seasonal pattern of dissolved iron concentrations at selected sites in the Eagle River watershed, Colorado, 1966-2007. 


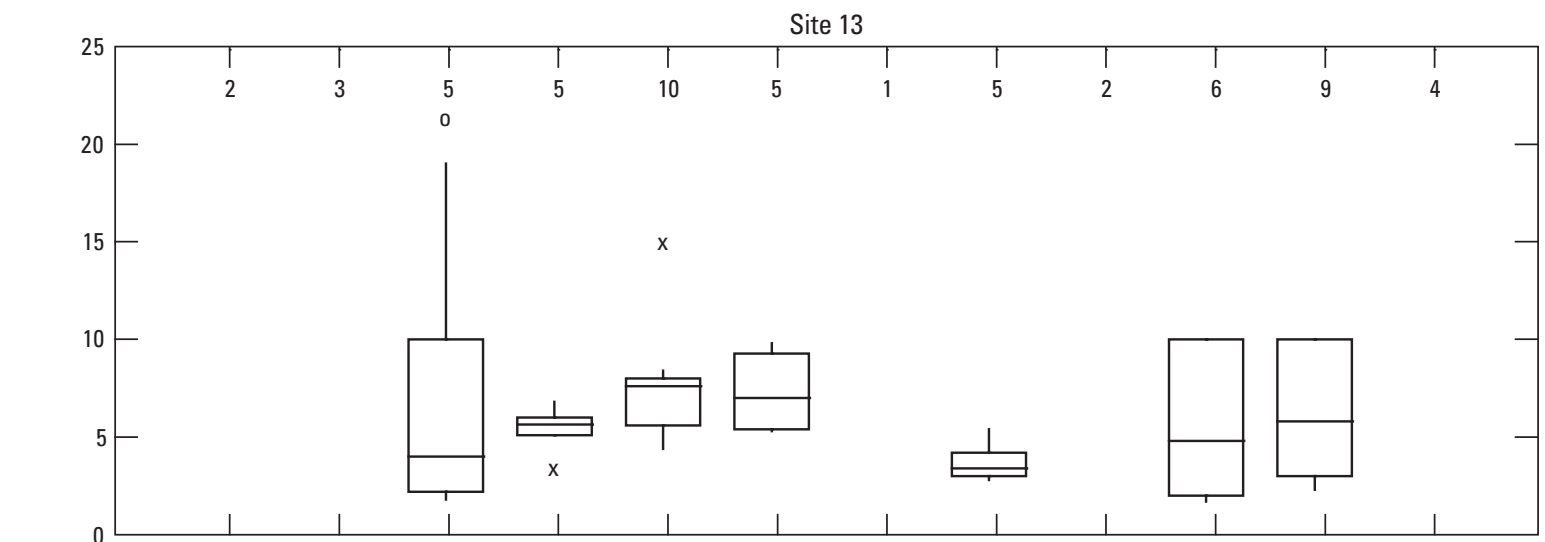

EXPLANATION

30 Number of values

$0 \quad$ Upper detached

x Upper outside Upper adjacent

90th percentile

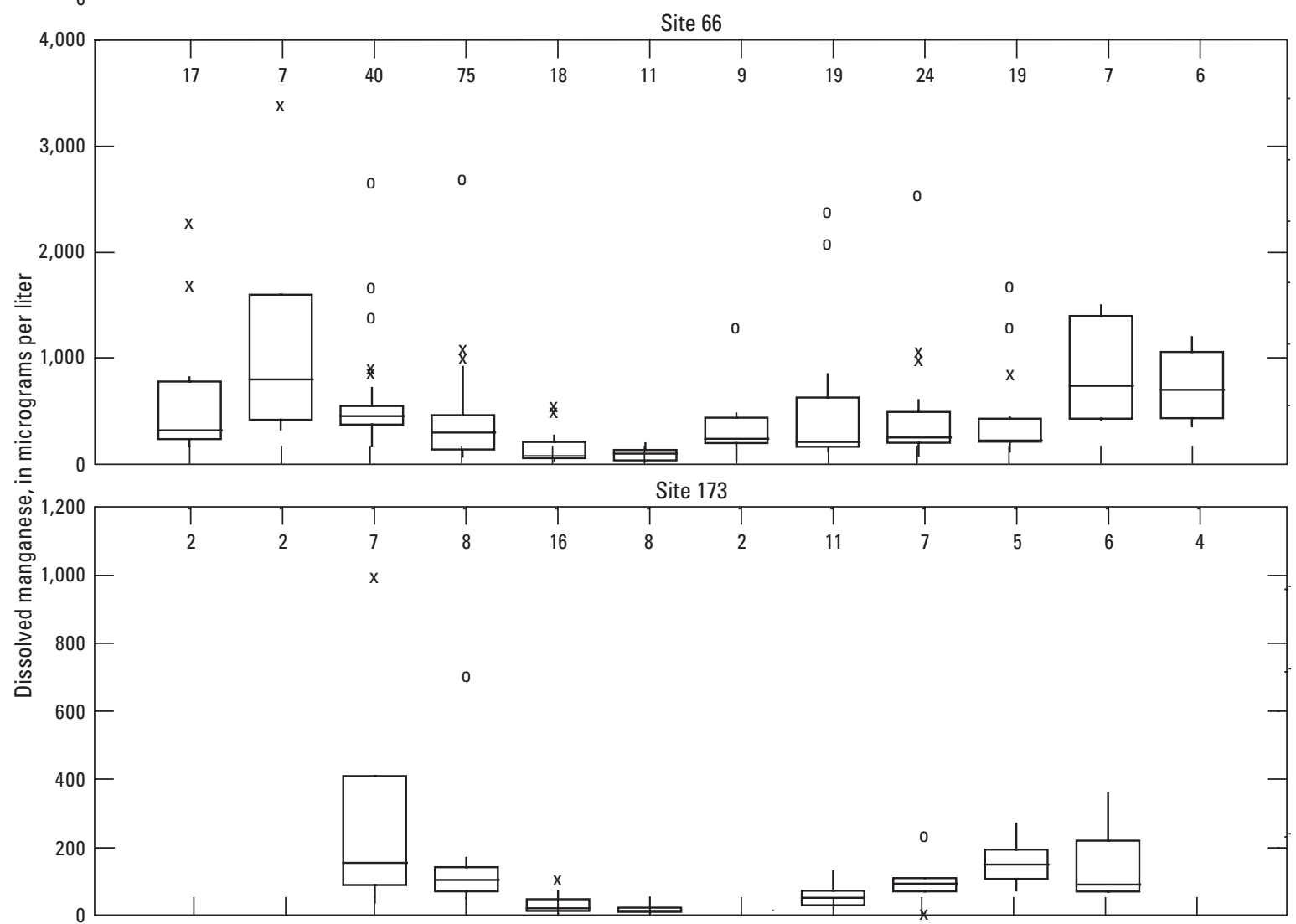

Site 224

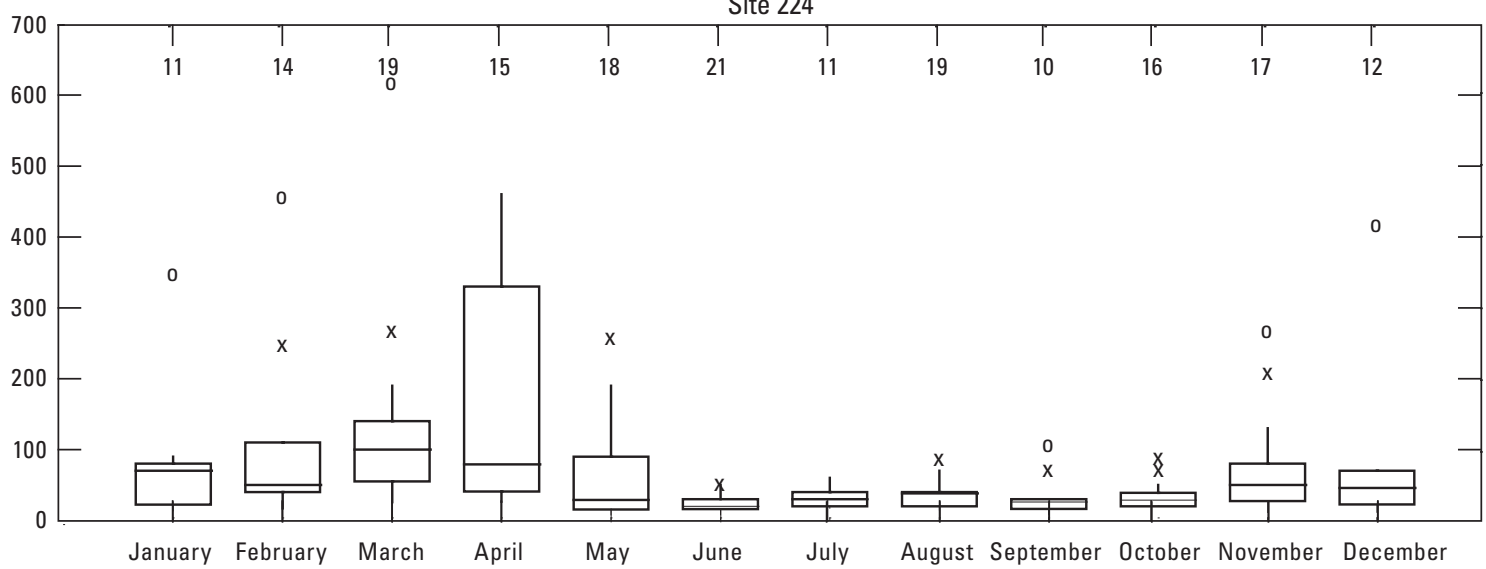

Figure 72. Seasonal pattern of dissolved manganese concentrations at selected sites in the Eagle River watershed, Colorado, $1970-2007$. 


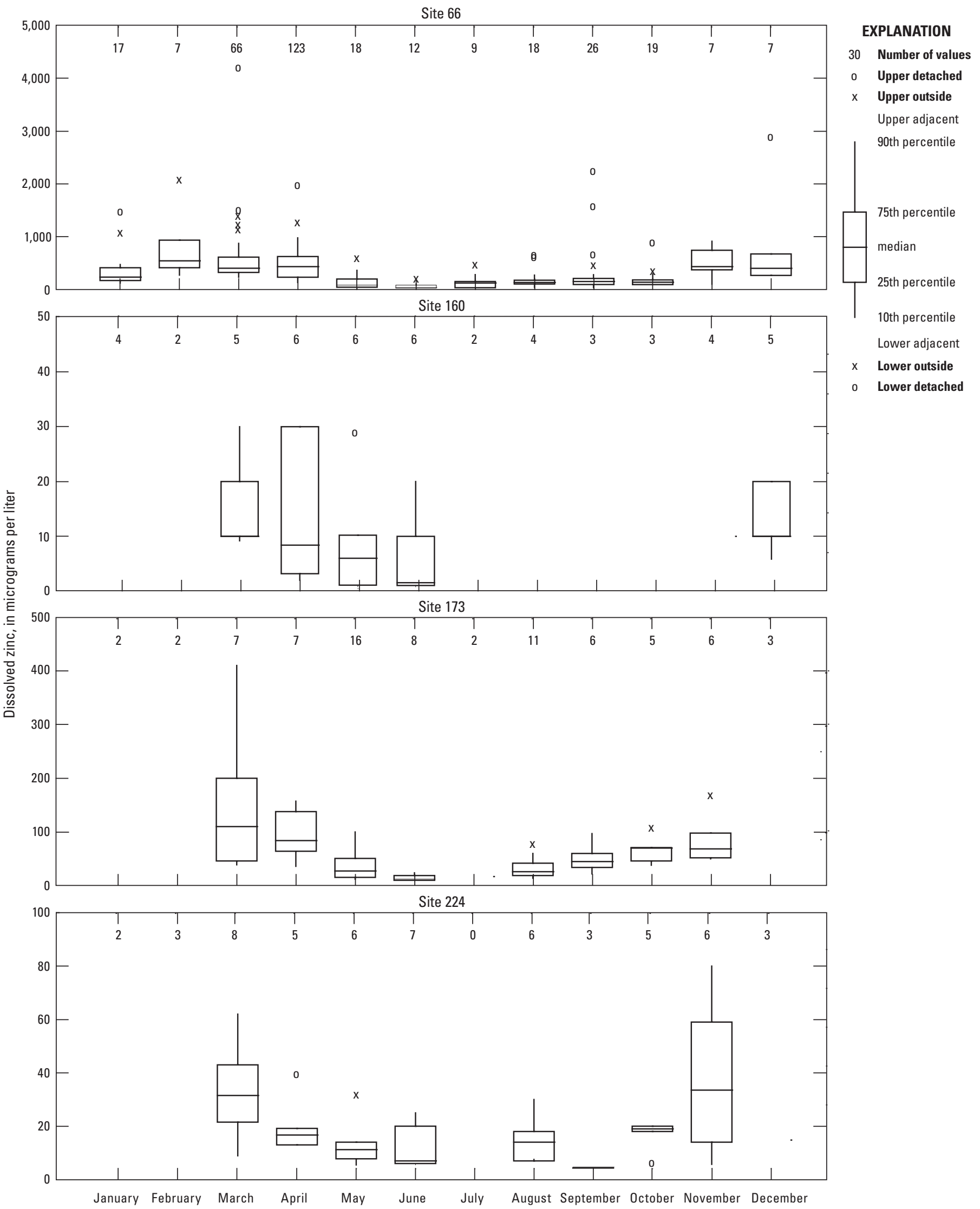

Figure 73. Seasonal patterns of dissolved zinc concentrations at selected sites in the Eagle River watershed, Colorado, 1966-2007. 


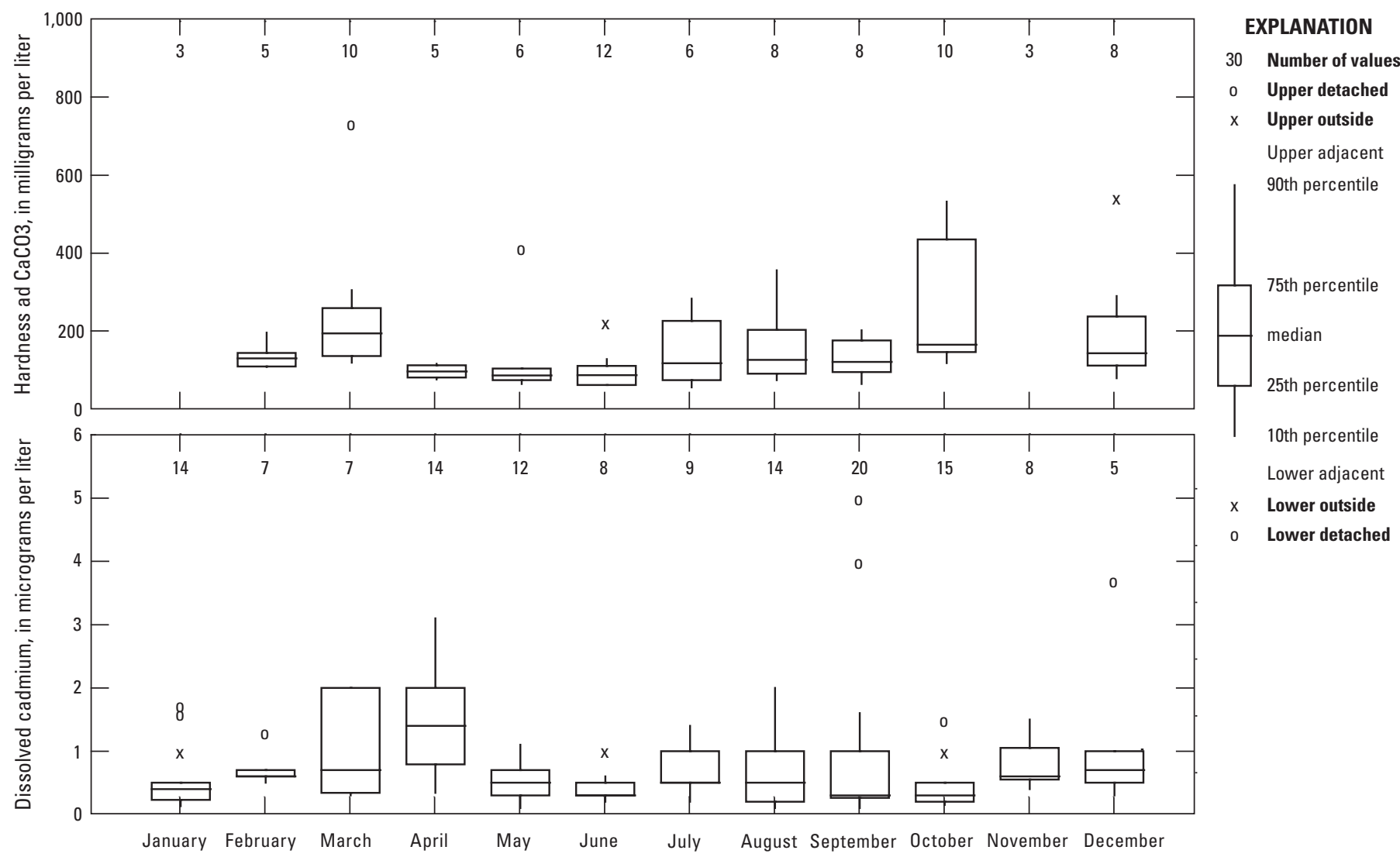

Figure 74. Seasonal pattern relation between trace elements concentrations and hardness at site 72 in the Eagle River watershed, Colorado.

Table value standards for uranium are designated by the CDPHE and applies to all reaches of the ERW. The acute and chronic TVS for uranium are site-specific based on hardness. There were no exceedances of the acute or chronic State standards for uranium out of 78 comparisons.

To better represent exceedances of State standards for trace-elements in the ERW as a whole, the combined number of samples with detected exceedances for any of the trace-element species State standards discussed was made into maps. Maps summarizing the spatial extent of acute and chronic State standards exceedances are presented in figures 76 and 77.

Extensive sampling for metals has been done in select areas of the ERW related to the Eagle Mine Superfund Site to address site remediation and compliance investigations. Sampling within specific reaches of the Eagle River and tributaries are closely spaced together making graphical presentation and resolution of the data difficult. Discussion of metals exceedances of State standards will focus on three sites that represent each of 3 conditions present within the ERW: (1) stream conditions upstream from the Eagle Mine area Upper Eagle subwatershed; (site 28); (2) stream conditions in the Eagle Mine area Upper Eagle subwatershed; (site 66); and (3) stream conditions downstream from the Eagle Mine area Middle
Eagle subwatershed; (site 173). Table 20 provides information on metals exceedances within the (Upper Eagle, Middle Eagle, Lower Eagle and Gore Creek) subwatersheds of the ERW.

State standards for cadmium are designated by the CDPHE. The chronic TVS for cadmium are site-specific based on hardness, except for segment $11(10 \mu \mathrm{g} / \mathrm{L}$ total recoverable cadmium). The chronic State standards for cadmium were exceeded less than 20 times over a period of several years for most sites in the ERW; approximately 20 percent (507 comparisons) of the total number of measurements $(2,548)$ exceeded the chronic State standards (table 20).

The acute TVS for cadmium are site-specific based on hardness, except for segment $11(10 \mu \mathrm{g} / \mathrm{L}$ total recoverable cadmium). Most sites in the ERW exceeded the acute State standard for cadmium fewer than 5 times; approximately 2 percent (44 comparisons) of the total number of measurements $(2,548)$ exceeded the acute State standard for cadmium (table 20). Additionally, an acute(trout) State standard exists for cadmium. Using this standard as a comparative reference approximately 3 percent ( 86 comparisons) of the total number of measurements $(2,548)$ exceeded the acute(trout) State standard (table 20). Figures 78-80 show the concentrations for dissolved cadmium through time at selected sites in comparison to State standards. 


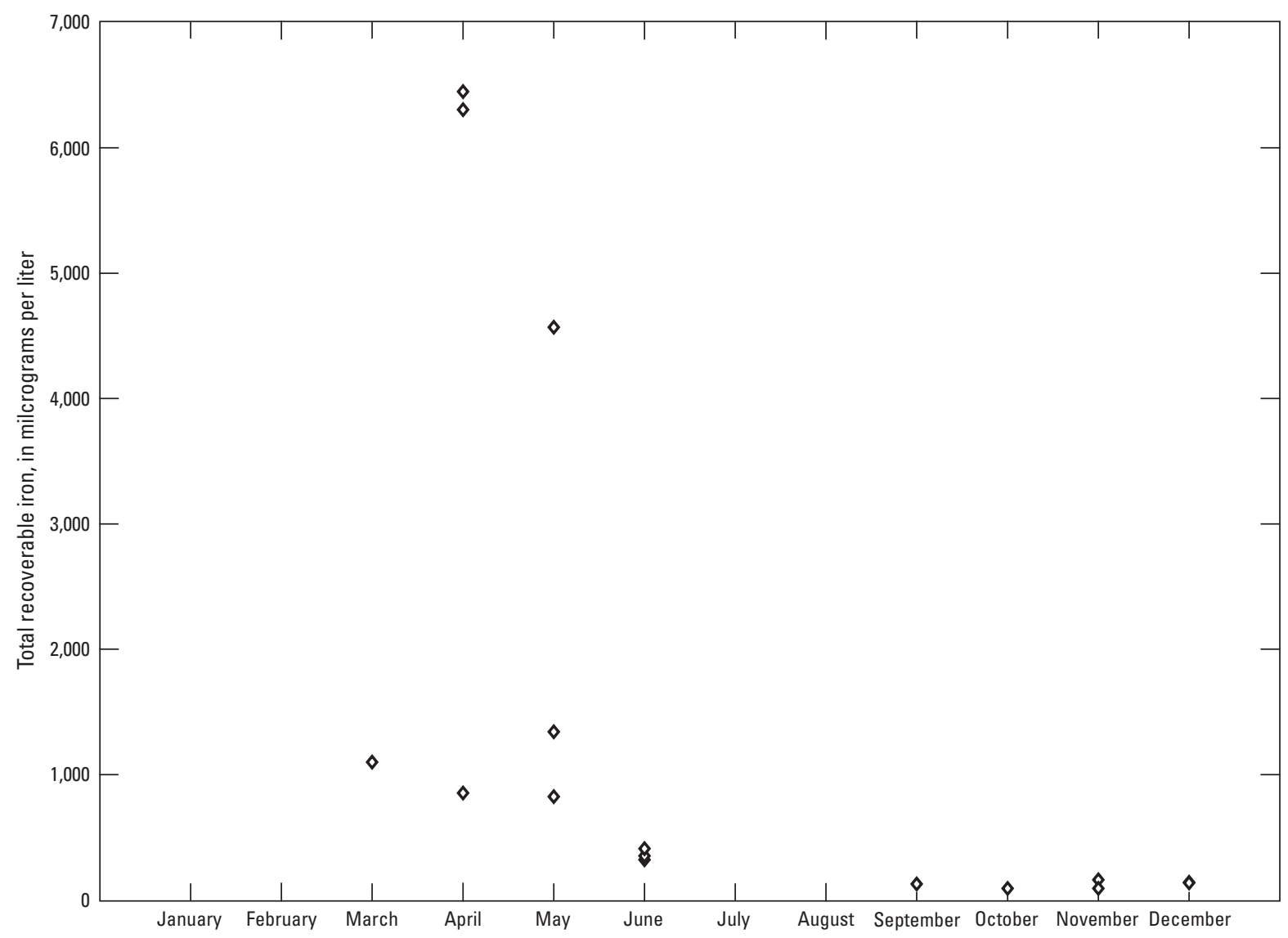

Figure 75. Seasonal patterns of total recoverable iron concentrations at site 224 in the Eagle River watershed, near Gypsum, Colorado. 


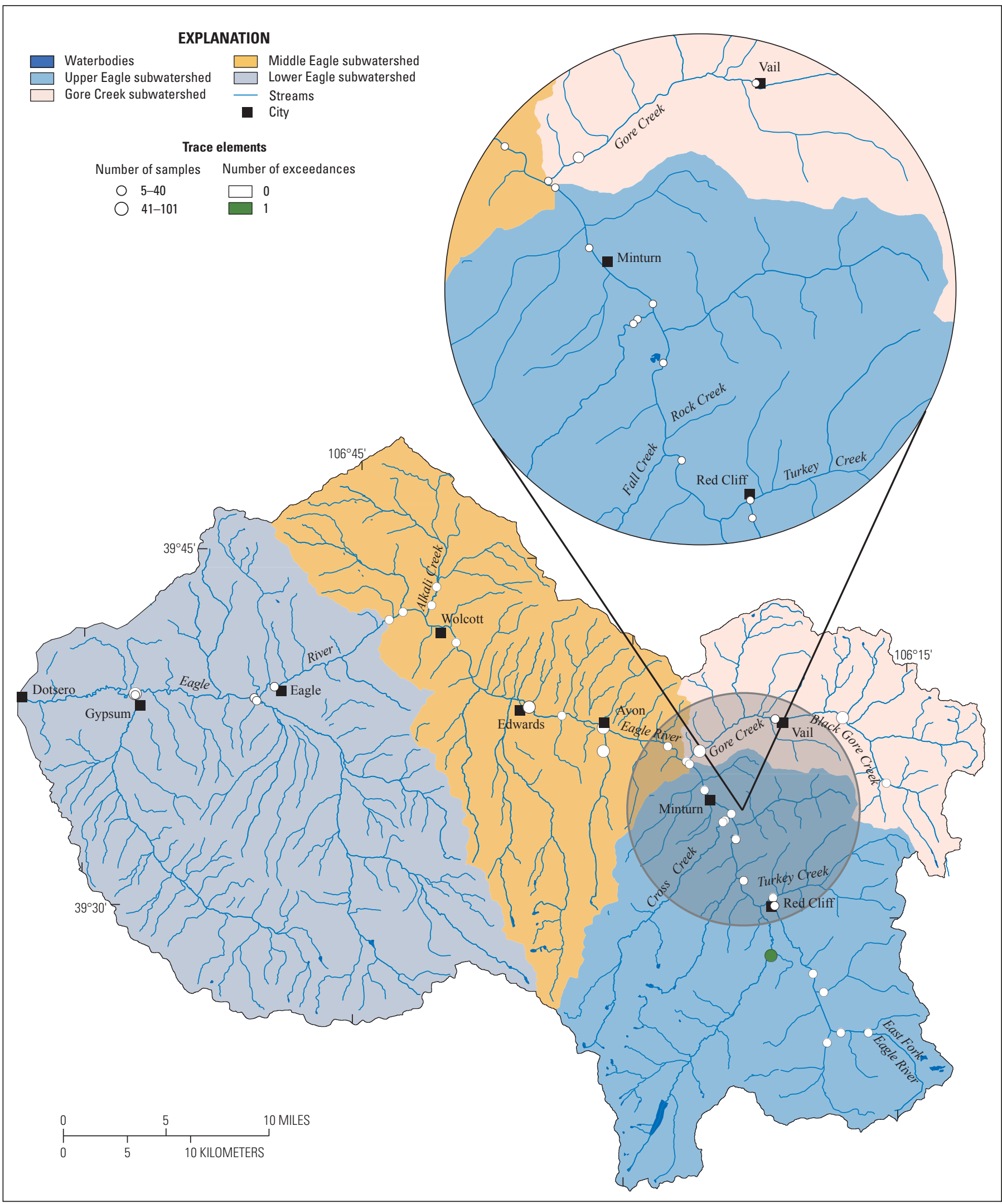

Base from U.S. Geological Survey digital data, 2007,

Universal Transverse Mercator projection

Zone 13

Figure 76. Number of exceedances of the Colorado acute water-quality standard for concentration of trace elements (dissolved arsenic, dissolved selenium, dissolved uranium) at selected sites in the Eagle River watershed, Colorado, 1971-2007. 


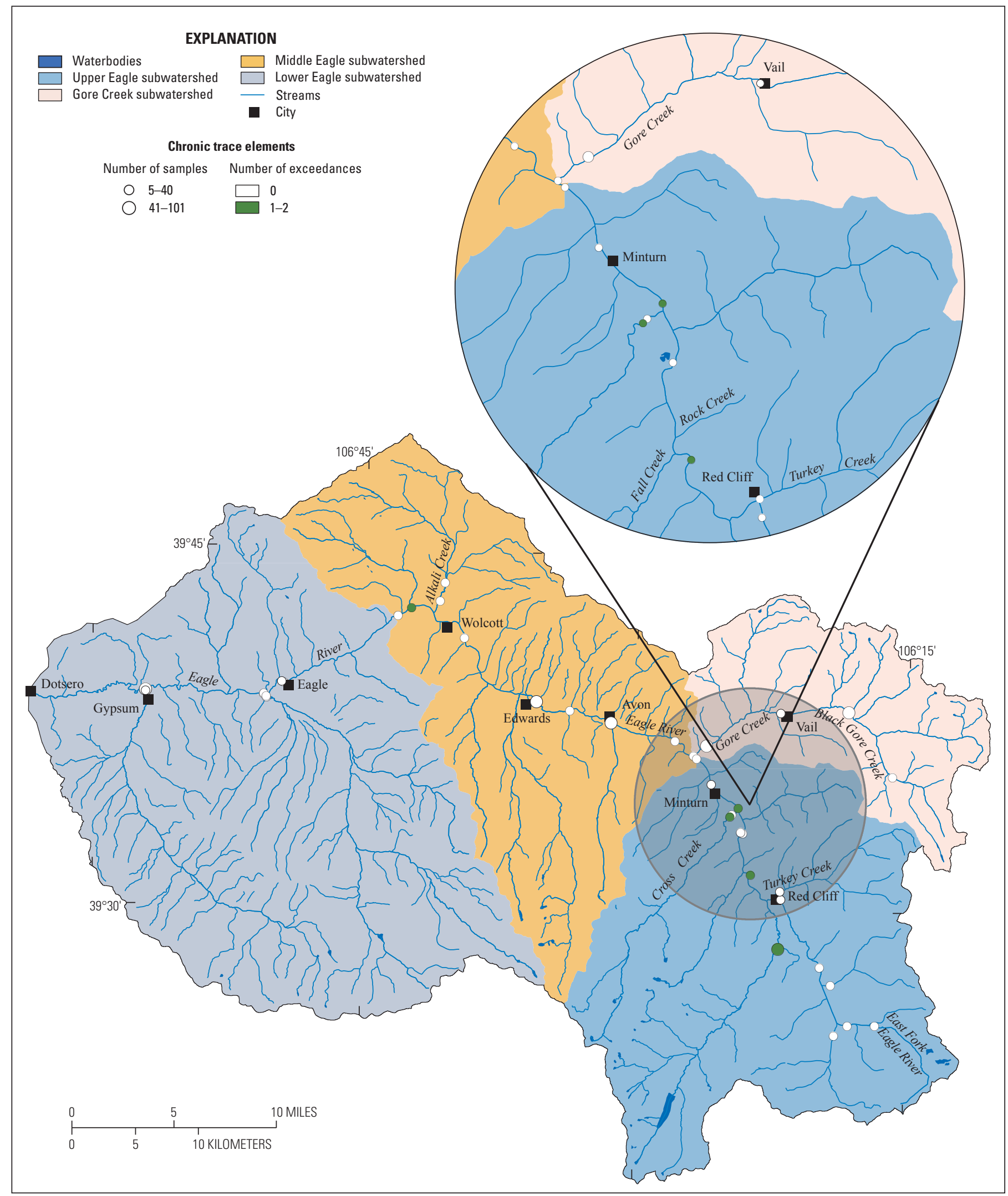

Base from U.S. Geological Survey digital data, 2007,

Universal Transverse Mercator projection

Zone 13

Figure 77. Number of exceedances of the Colorado chronic water-quality standard for concentration of trace elements (dissolved arsenic, dissolved selenium, dissolved uranium) at selected sites in the Eagle River watershed, Colorado, 1968-2001. 
Table 20. Summary of water-quality standard exceedances for trace elements in the Eagle River watershed by subwatershed.

[Exceedances based on dissolved concentrations, unless otherwise noted; No., number; --, no data]

\begin{tabular}{|c|c|c|c|c|c|c|}
\hline $\begin{array}{l}\text { Constituent } \\
\text { or property }\end{array}$ & $\begin{array}{c}\text { No. of } \\
\text { censored } \\
\text { values }\end{array}$ & $\begin{array}{c}\text { No. of } \\
\text { samples }\end{array}$ & $\begin{array}{c}\text { No. of } \\
\text { standard } \\
\text { exceedances }\end{array}$ & $\begin{array}{c}\text { Percent of } \\
\text { standard } \\
\text { exceedances }\end{array}$ & $\begin{array}{l}\text { Minimum } \\
\text { date }\end{array}$ & $\begin{array}{l}\text { Maximum } \\
\text { date }\end{array}$ \\
\hline \multicolumn{7}{|c|}{ Gore Creek subwatershed } \\
\hline Aluminum, chronic & 2 & 22 & 10 & 45 & May-19-99 & Oct-16-01 \\
\hline Arsenic, acute & 101 & 103 & 0 & 0 & -- & -- \\
\hline Arsenic, chronic & 101 & 103 & 0 & 0 & -- & -- \\
\hline Cadmium, chronic & 201 & 219 & 4 & 2 & Mar-14-89 & Jun-18-97 \\
\hline Copper, acute & 218 & 304 & 1 & $<1$ & Sep-06-89 & Sep-06-89 \\
\hline Copper, chronic & 218 & 304 & 5 & 2 & Sep-06-89 & Aug-30-07 \\
\hline Iron, chronic ${ }^{1}$ & 205 & 731 & 8 & 1 & Apr-25-89 & Jun-04-03 \\
\hline Lead, acute & 230 & 247 & 0 & 0 & -- & -- \\
\hline Lead, chronic & 230 & 247 & 0 & 0 & -- & -- \\
\hline Selenium, acute & 56 & 70 & 0 & 0 & -- & -- \\
\hline Selenium, chronic & 56 & 70 & 0 & 0 & -- & -- \\
\hline Silver, acute & 133 & 139 & 0 & 0 & -- & -- \\
\hline Silver, chronic & 133 & 139 & 0 & 0 & -- & -- \\
\hline Silver, chronic (trout) & 133 & 139 & 3 & 2 & Dec-28-88 & Sep-20-89 \\
\hline Uranium, acute & 13 & 37 & 0 & 0 & -- & -- \\
\hline Uranium, chronic & 13 & 37 & 0 & 0 & -- & -- \\
\hline Zinc, acute & 257 & 425 & 0 & 0 & -- & -- \\
\hline Zinc, chronic & 257 & 425 & 0 & 0 & -- & -- \\
\hline \multicolumn{7}{|c|}{ Upper Eagle subwatershed } \\
\hline Aluminum, acute & 0 & 17 & 0 & 0 & -- & -- \\
\hline Aluminum, chronic & 0 & 17 & 17 & 100 & Oct-11-68 & Apr-03-01 \\
\hline Lead, acute & 527 & 1,780 & 0 & 0 & -- & -- \\
\hline Lead, chronic & 527 & 1,780 & 190 & 11 & Oct-11-68 & Mar-30-99 \\
\hline Manganese, acute & 106 & 1,926 & 39 & 2 & Dec-13-88 & Mar-10-93 \\
\hline Manganese, chronic & 106 & 1,926 & 96 & 5 & Oct-27-70 & Mar-07-95 \\
\hline Nickel, acute & 8 & 23 & 0 & 0 & -- & -- \\
\hline Nickel, chronic & 8 & 23 & 0 & 0 & -- & -- \\
\hline Selenium, acute & 113 & 153 & 0 & 0 & -- & -- \\
\hline Selenium, chronic & 113 & 153 & 4 & 3 & Jun-28-68 & Oct-27-70 \\
\hline Silver, acute & 170 & 238 & 7 & 3 & Oct-27-70 & Mar-23-01 \\
\hline Silver, chronic & 170 & 238 & 11 & 5 & Oct-27-70 & Mar-23-01 \\
\hline Silver, chronic (trout) & 170 & 238 & 30 & 13 & Oct-27-70 & Apr-17-02 \\
\hline Uranium, acute & 4 & 15 & 0 & 0 & -- & -- \\
\hline Uranium, chronic & 4 & 15 & 0 & 0 & -- & -- \\
\hline Zinc, acute & 129 & 2,292 & 583 & 25 & Oct-05-66 & Oct-23-06 \\
\hline Zinc, chronic & 129 & 2,292 & 610 & 27 & Oct-05-66 & Oct-23-06 \\
\hline
\end{tabular}


Table 20. Summary of water-quality standard exceedances for trace elements in the Eagle River watershed by subwatershed.-Continued

[Exceedances based on dissolved concentrations, unless otherwise noted; No., number; --, no data]

\begin{tabular}{|c|c|c|c|c|c|c|}
\hline $\begin{array}{l}\text { Constituent } \\
\text { or property }\end{array}$ & $\begin{array}{c}\text { No. of } \\
\text { censored } \\
\text { values }\end{array}$ & $\begin{array}{c}\text { No. of } \\
\text { samples }\end{array}$ & $\begin{array}{c}\text { No. of } \\
\text { standard } \\
\text { exceedances }\end{array}$ & $\begin{array}{c}\text { Percent of } \\
\text { standard } \\
\text { exceedances }\end{array}$ & $\begin{array}{l}\text { Minimum } \\
\text { date }\end{array}$ & $\begin{array}{c}\text { Maximum } \\
\text { date }\end{array}$ \\
\hline \multicolumn{7}{|c|}{ Middle Eagle subwatershed } \\
\hline Aluminum, acute & 4 & 30 & 0 & 0 & -- & -- \\
\hline Aluminum, chronic & 4 & 30 & 8 & 27 & Dec-04-00 & Sep-11-03 \\
\hline Arsenic, acute & 46 & 51 & 0 & 0 & -- & -- \\
\hline Arsenic, chronic & 46 & 51 & 0 & 0 & -- & -- \\
\hline Cadmium, acute & 171 & 317 & 0 & 0 & -- & -- \\
\hline Cadmium, acute (trout) & 171 & 317 & 4 & 1 & Sep-14-89 & Mar-08-95 \\
\hline Cadmium, chronic & 171 & 317 & 32 & 10 & Oct-26-88 & Sep-09-98 \\
\hline Copper, acute & 153 & 338 & 0 & 0 & -- & -- \\
\hline Copper, chronic & 153 & 338 & 3 & $<1$ & Jan-10-91 & Sep-14-99 \\
\hline Iron, chronic ${ }^{1}$ & 50 & 448 & 58 & 13 & May-19-76 & Aug-28-07 \\
\hline Lead, acute & 259 & 331 & 0 & 0 & -- & -- \\
\hline Lead, chronic & 259 & 331 & 0 & 0 & -- & -- \\
\hline Manganese, acute & 41 & 385 & 3 & $<1$ & Jan-18-90 & Mar-15-90 \\
\hline Manganese, chronic & 41 & 385 & 10 & 3 & Mar-14-89 & Mar-15-90 \\
\hline Nickel, acute & 4 & 20 & 0 & 0 & -- & -- \\
\hline Nickel, chronic & 4 & 20 & 0 & 0 & -- & -- \\
\hline Selenium, acute & 99 & 139 & 0 & 0 & -- & -- \\
\hline Selenium, chronic & 99 & 139 & 1 & $<1$ & Feb-15-01 & Feb-15-01 \\
\hline Silver, acute & 153 & 158 & 0 & 0 & -- & -- \\
\hline Silver, chronic & 153 & 158 & 0 & 0 & -- & -- \\
\hline Silver, chronic (trout) & 153 & 158 & 0 & 0 & -- & -- \\
\hline Uranium, acute & 1 & 13 & 0 & 0 & -- & -- \\
\hline Uranium, chronic & 1 & 13 & 0 & 0 & -- & -- \\
\hline Zinc, acute & 32 & 340 & 45 & 13 & Jan-5-77 & Feb-18-97 \\
\hline Zinc, chronic & 32 & 340 & 56 & 16 & Jan-5-77 & Feb-18-97 \\
\hline \multicolumn{7}{|c|}{ Lower Eagle subwatershed } \\
\hline Aluminum, acute & 0 & 35 & 4 & 11 & Aug-29-00 & May-23-03 \\
\hline Aluminum, chronic & 0 & 35 & 28 & 80 & Dec-02-98 & May-23-03 \\
\hline Arsenic, acute & 87 & 108 & 0 & 0 & -- & -- \\
\hline Arsenic, chronic & 87 & 108 & 0 & 0 & -- & -- \\
\hline Cadmium, acute & 172 & 207 & 0 & 0 & -- & -- \\
\hline Cadmium, acute (trout) & 172 & 207 & 0 & 0 & -- & -- \\
\hline Cadmium, chronic & 172 & 207 & 2 & 1 & Aug-18-86 & Apr-28-92 \\
\hline Copper, acute & 113 & 232 & 0 & 0 & -- & -- \\
\hline Copper, chronic & 113 & 232 & 0 & 0 & -- & -- \\
\hline Iron, chronic ${ }^{1}$ & 114 & 555 & 31 & 6 & May-18-76 & Apr-10-06 \\
\hline Lead, acute & 235 & 258 & 0 & 0 & -- & - \\
\hline Lead, chronic & 235 & 258 & 1 & $<1$ & Nov-20-69 & Nov-20-69 \\
\hline Manganese, acute & 23 & 405 & 0 & 0 & -- & -- \\
\hline Manganese, chronic & 23 & 405 & 0 & 0 & -- & -- \\
\hline Nickel, acute & 28 & 34 & 0 & 0 & -- & -- \\
\hline Nickel, chronic & 28 & 34 & 0 & 0 & -- & -- \\
\hline Selenium, acute & 94 & 148 & 0 & 0 & -- & -- \\
\hline Selenium, chronic & 94 & 148 & 0 & 0 & -- & -- \\
\hline Silver, acute & 95 & 97 & 0 & 0 & -- & -- \\
\hline Silver, chronic & 95 & 97 & 0 & 0 & -- & -- \\
\hline Uranium, acute & 0 & 13 & 0 & 0 & -- & -- \\
\hline Uranium, chronic & 0 & 13 & 0 & 0 & -- & -- \\
\hline Zinc, acute & 23 & 246 & 0 & 0 & -- & -- \\
\hline Zinc, chronic & 23 & 246 & 0 & 0 & -- & -- \\
\hline
\end{tabular}

${ }^{1}$ Counts include both total recoverable and dissolved iron. 
The chronic TVS for copper is site-specific based on hardness except reach 11 (200 $\mu \mathrm{g} / \mathrm{L}$ total recoverable) (fig. 12). The chronic State standard for copper was exceeded less than 10 times over a period of several years for most sites in the ERW; approximately 4 percent (104 comparisons) of the total number of measurements $(2,702)$ exceeded the chronic State standard (table 20).

The acute TVS for copper set by the CDPHE is site specific based on hardness. Reach 11 does not have an acute TVS for copper (fig. 12). The acute TVS was exceeded fewer than 10 times at most sites within the ERW; approximately 2 percent (60 comparisons) of the total number of measurements $(2,702)$ exceeded the acute TVS (table 20). Figures 81-83 show the concentrations for dissolved copper through time at selected sites in comparison to State standards.

Chronic State standards for iron are designated by the CDPHE for both dissolved iron and total recoverable iron. Reach 11 has no State standard for iron (fig. 12). For total recoverable iron, the chronic State standard is $1,000 \mu \mathrm{g} / \mathrm{L}$. The chronic State standard for total recoverable iron was exceeded less than 20 times over a period of several years for most sites in the ERW; 26 percent (421 comparisons) of the total number of measurements $(1,596)$ exceeded the chronic State standard.

For dissolved iron, the chronic State standard is $300 \mu \mathrm{g} / \mathrm{L}$. Most sites in the ERW exceeded the chronic State standard fewer than 20 times; 14 percent (363 comparisons) of the total number of measurements $(2,624)$ exceeded the chronic State standard (table 20). Figures 84-89 show the concentrations for dissolved iron through time at selected sites in comparison to the State standard. Figures 87-89 show the concentrations for total iron through time at selected sites in comparison to the State standard.

Standards for manganese are designated by the CDPHE. The chronic State standard for manganese is determined by the less restrictive value of either the TVS, which is site-specific based on hardness, or $50 \mu \mathrm{g} / \mathrm{L}$ dissolved for all reaches of the ERW except for reach $11(200 \mu \mathrm{g} / \mathrm{L}$ total recoverable) (fig. 12). The manganese State standards were exceeded less than 20 times over a period of several years for most sites in the ERW; approximately 3 percent (106 comparisons) of the total number of measurements $(3,241)$ exceeded the chronic State standards.

The acute State standard for manganese is the less restrictive value of either the TVS or $50 \mu \mathrm{g} / \mathrm{L}$ dissolved for all reaches of the ERW except for reach $11(200 \mu \mathrm{g} / \mathrm{L}$ total recoverable) (fig. 12). Most sites in the ERW exceeded the acute State standards for manganese fewer than 15 times; approximately 1 percent (42 comparisons) of the total number of measurements $(3,241)$ exceeded the acute State standard (table 20). Figures 90-92 show the concentrations for dissolved manganese through time at selected sites in comparison to acute and chronic State standards.

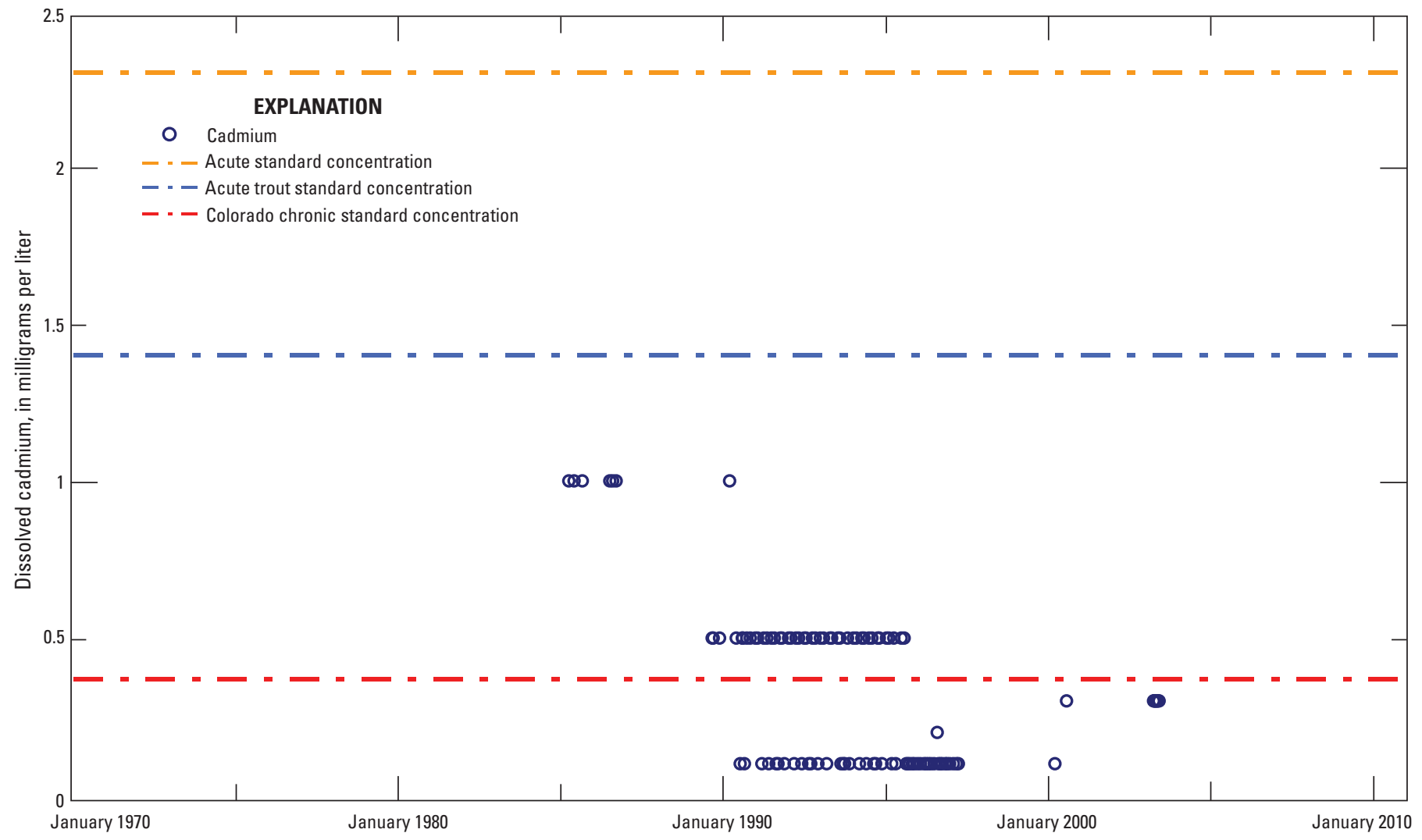

Figure 78. Temporal distribution of dissolved cadmium concentrations at site 28, in the Eagle River watershed, Colorado, $1984-2003$. 


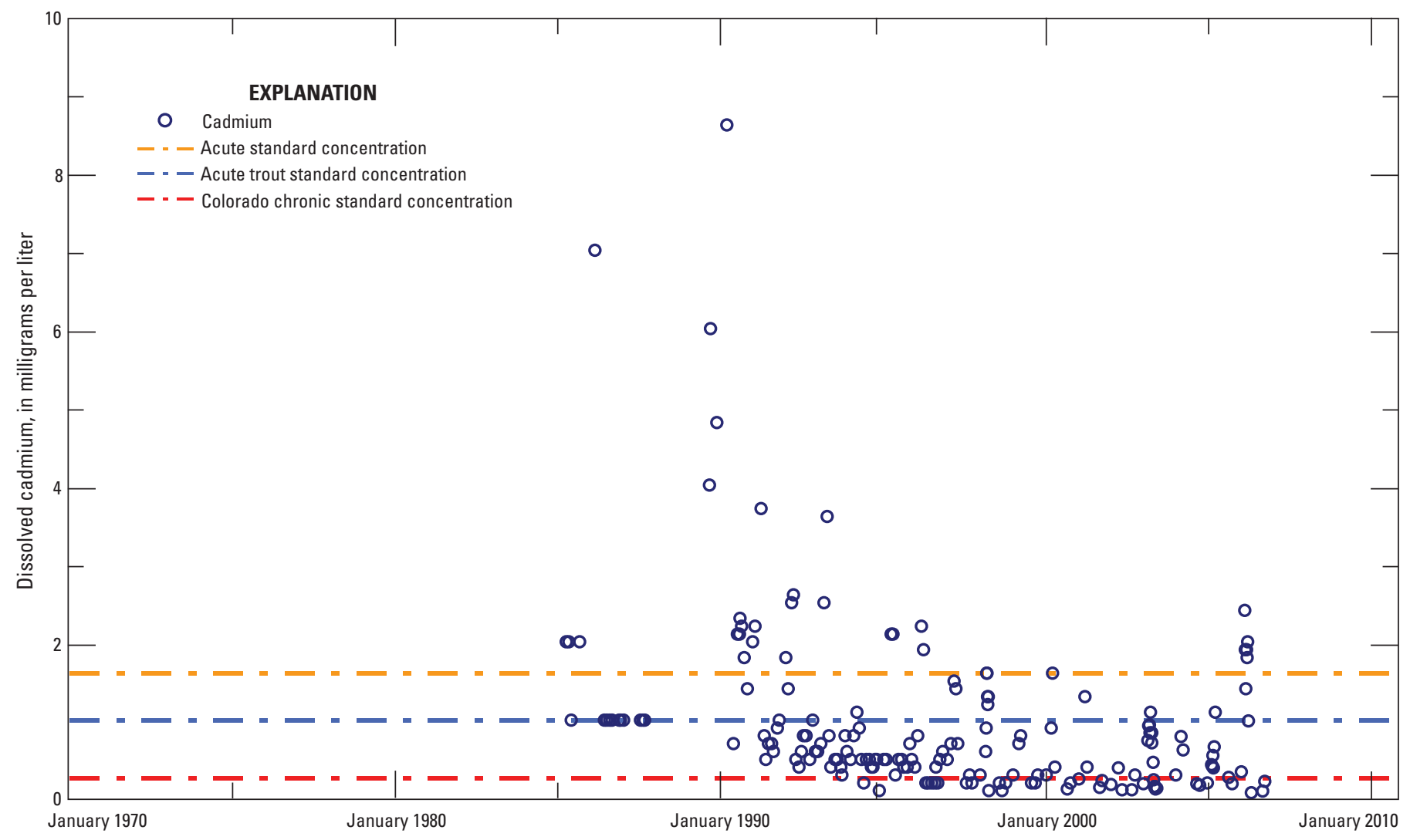

Figure 79. Temporal distribution of dissolved cadmium concentrations at site 66, in the Eagle River watershed, Colorado, $1985-2007$.

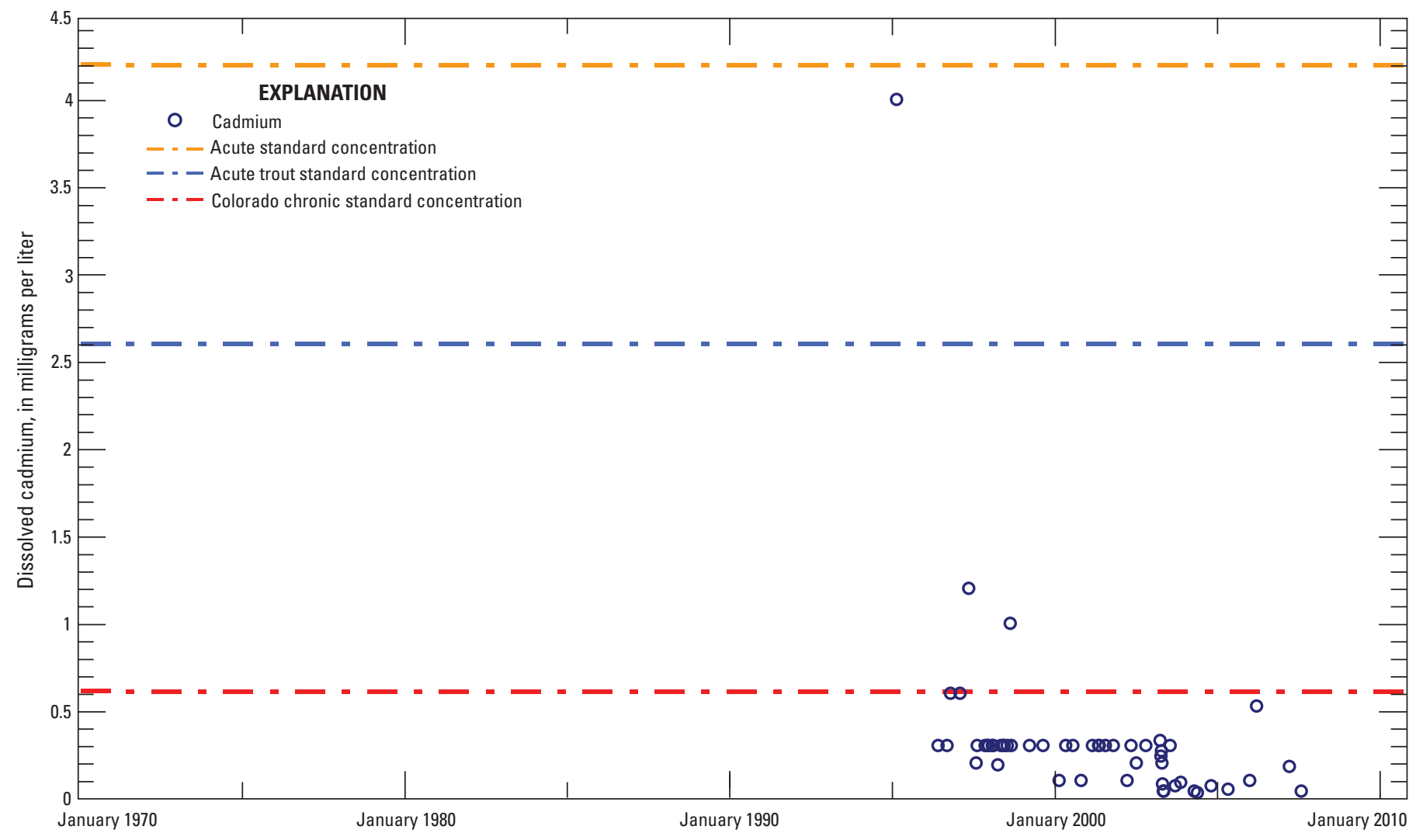

Figure 80. Temporal distribution of dissolved cadmium concentrations at site 173, in the Eagle River watershed, $1997-2007$. 


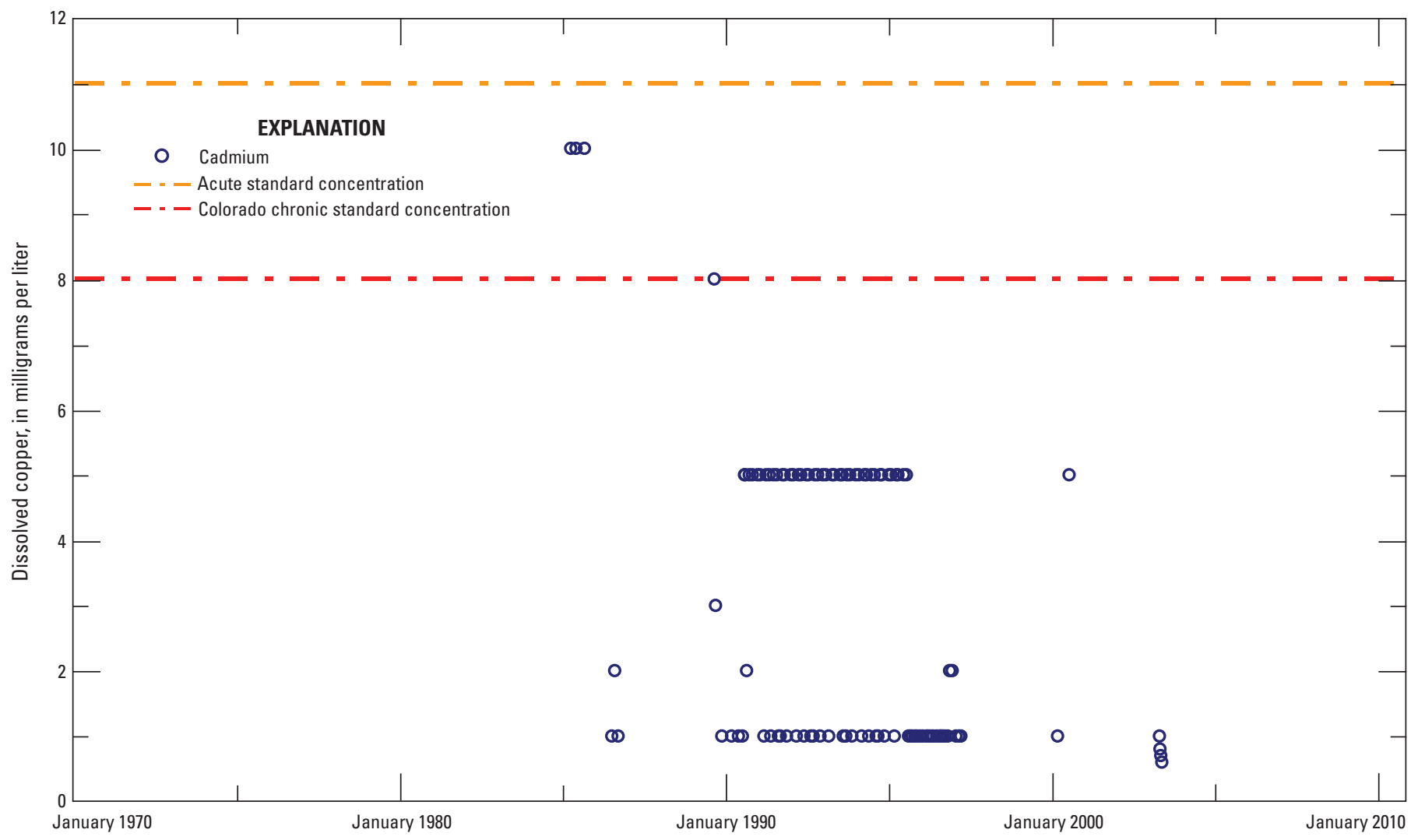

Figure 81. Temporal distribution of dissolved copper concentrations at site 28, in the Eagle River watershed, Colorado, $1985-2003$.

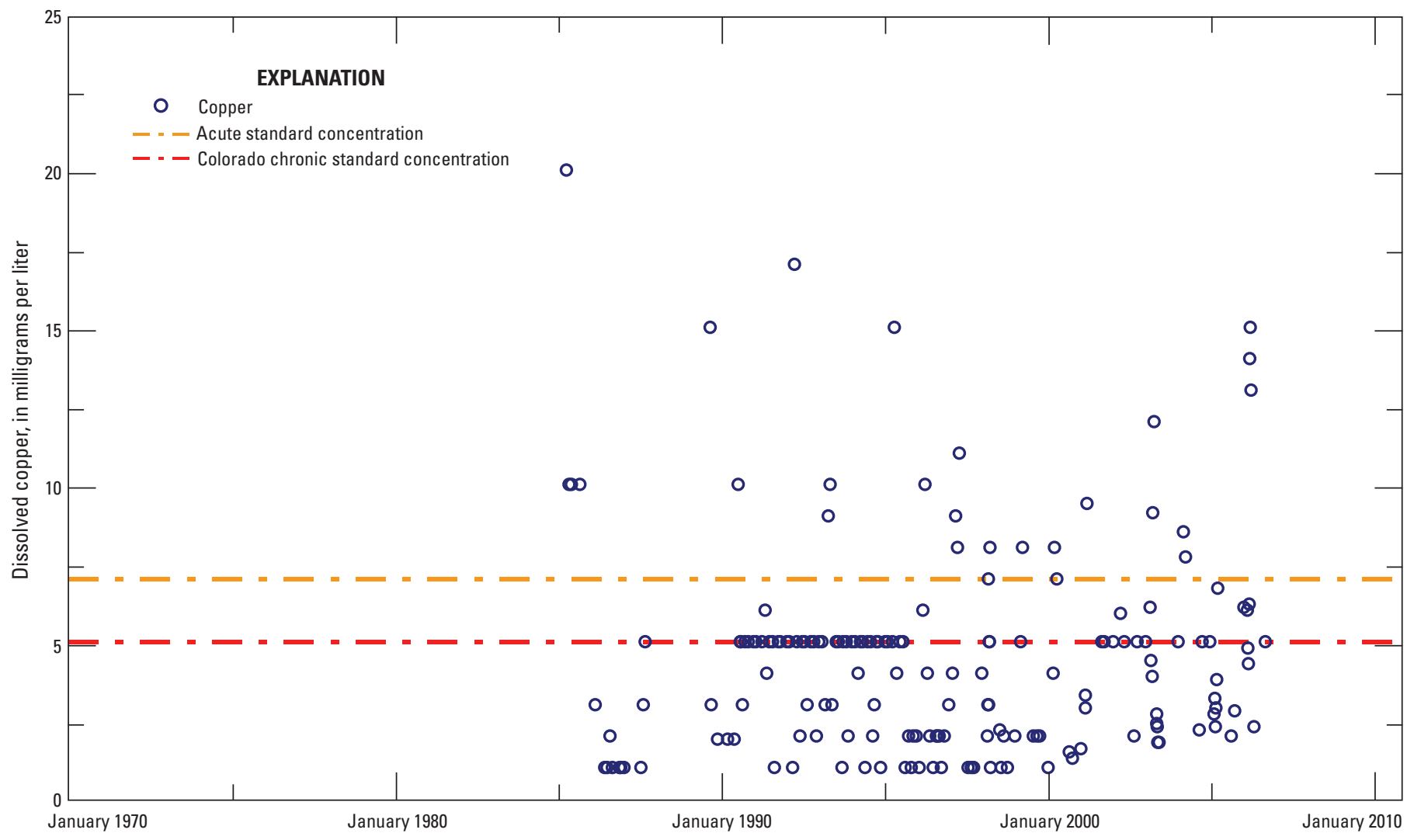

Figure 82. Temporal distribution of dissolved copper concentrations at site 66, in the Eagle River watershed, Colorado, $1981-2007$. 


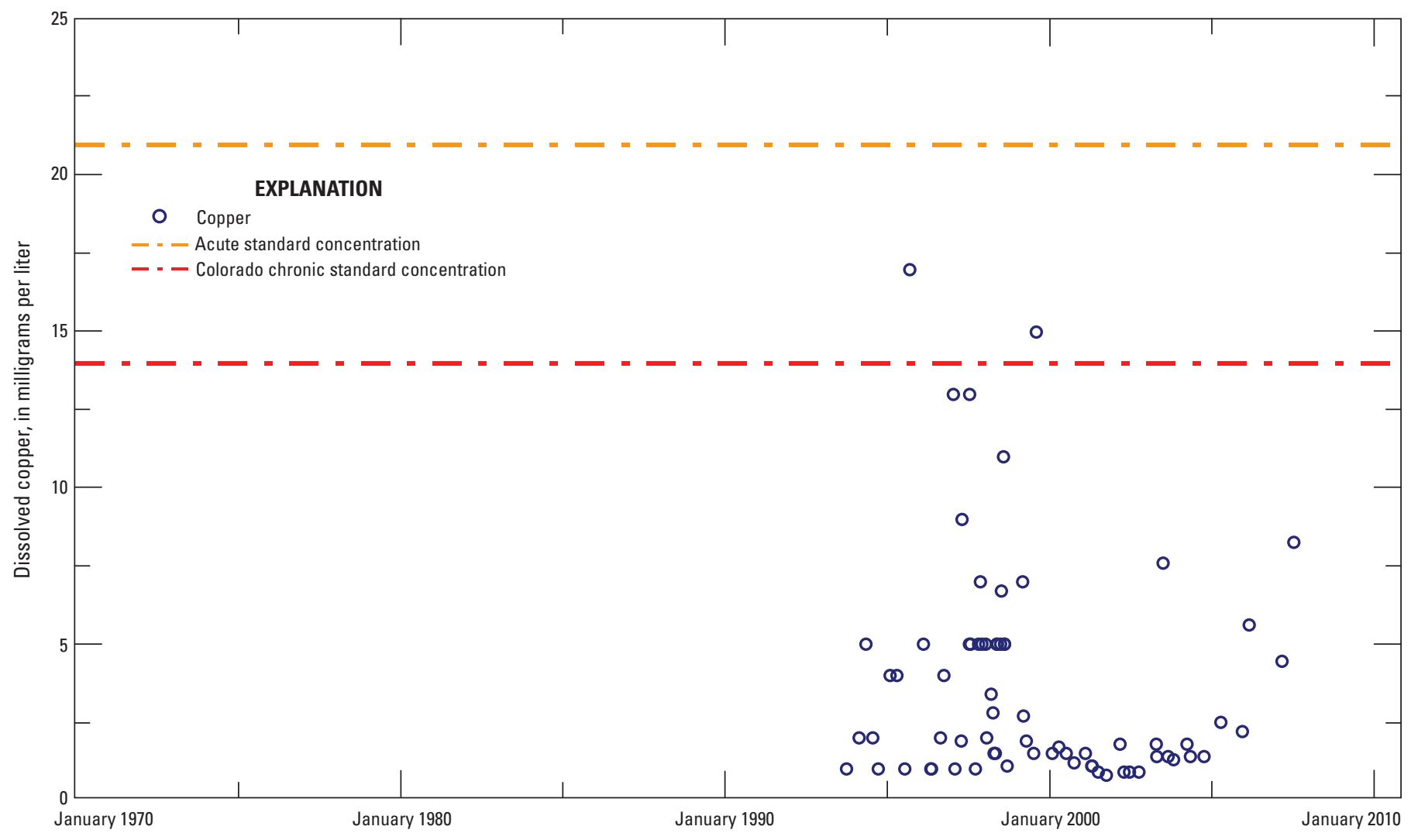

Figure 83. Temporal distribution of dissolved copper concentrations at site 173, in the Eagle River watershed, Colorado, $1994-2007$.

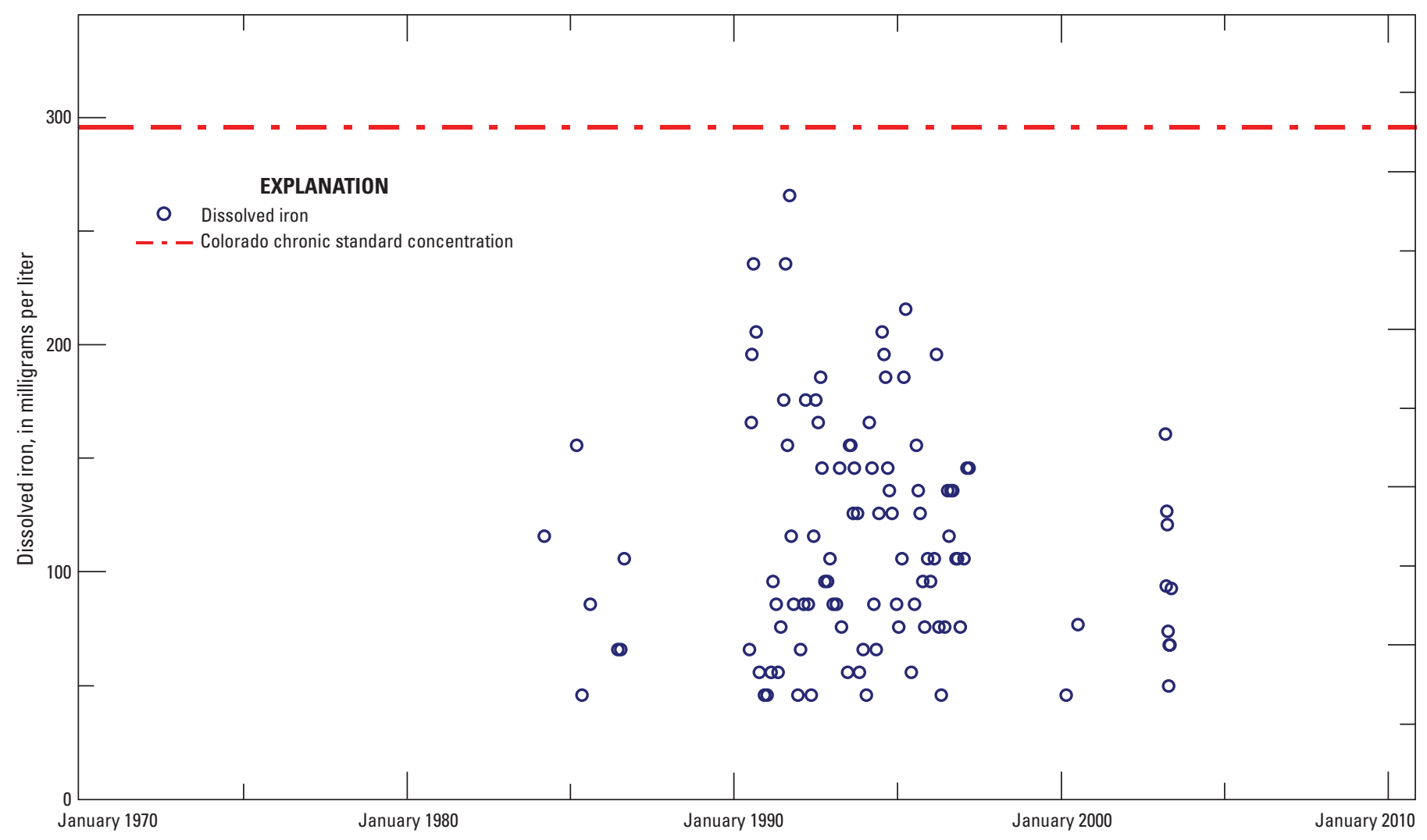

Figure 84. Temporal distribution of dissolved iron concentrations at site 28, in the Eagle River watershed, Colorado, 1984-2003. 


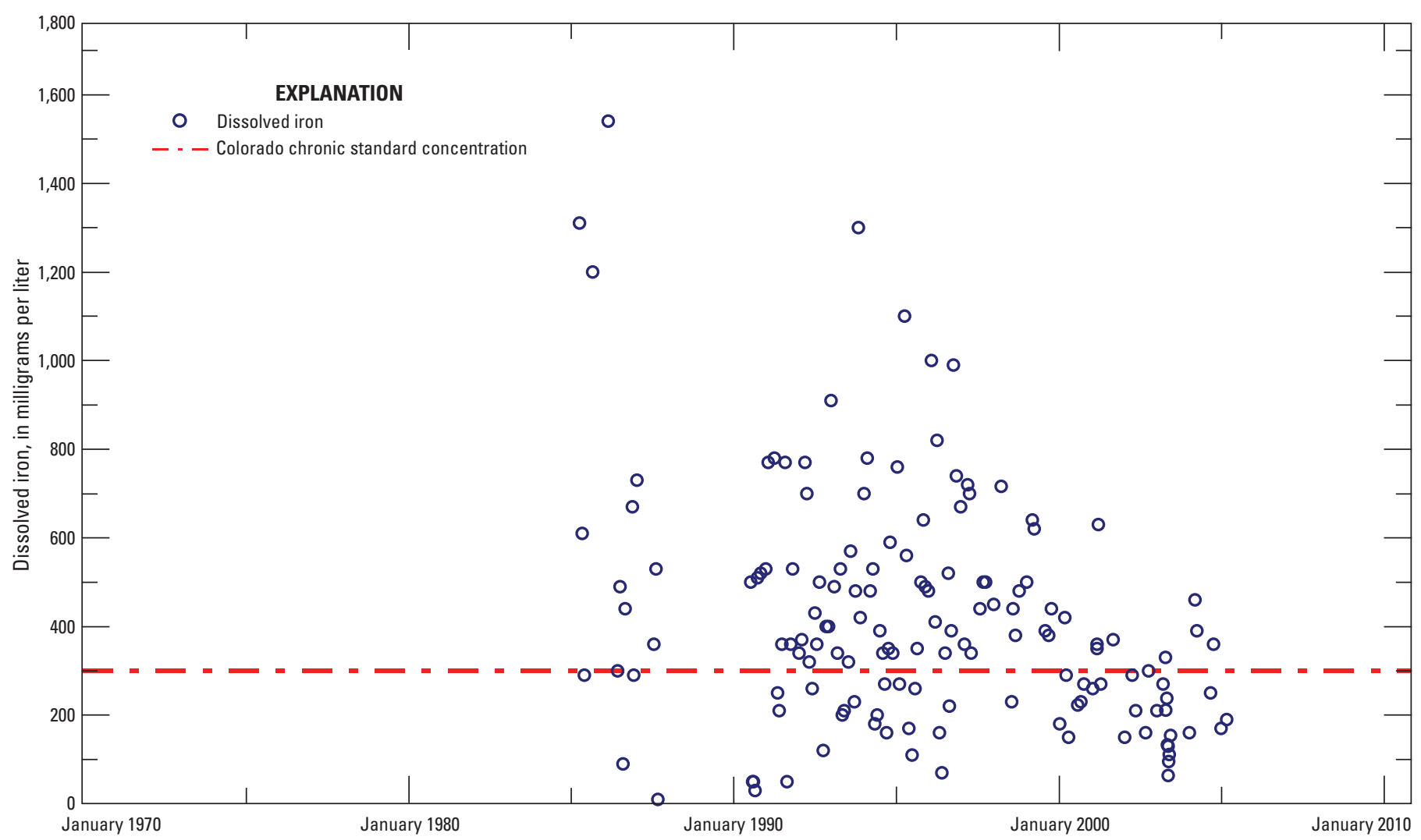

Figure 85. Temporal distribution of dissolved iron concentrations at site 66, in the Eagle River watershed, Colorado, 1985-2005.

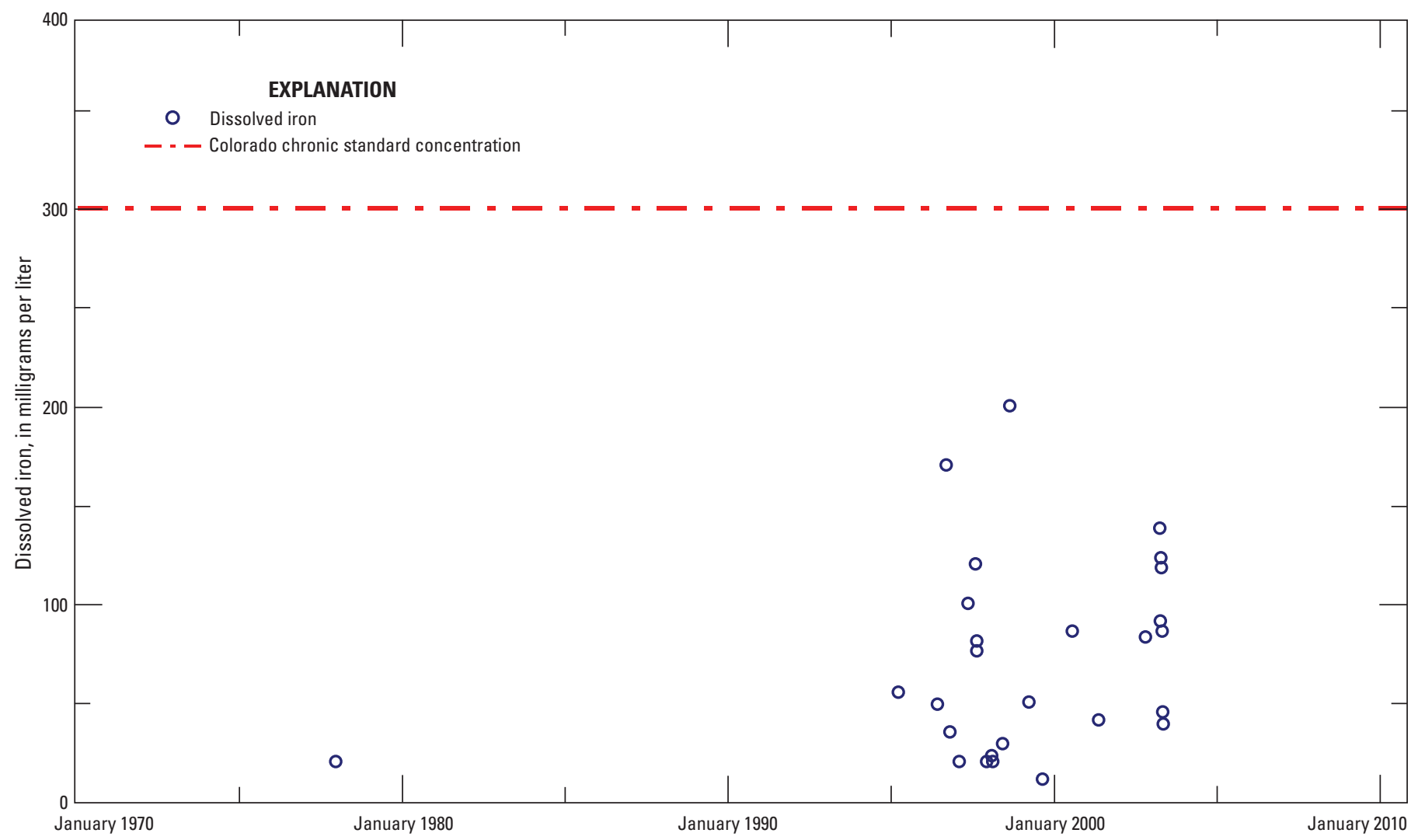

Figure 86. Temporal distribution of dissolved iron concentrations at site 173, in the Eagle River watershed, Colorado, 1978-2003. 


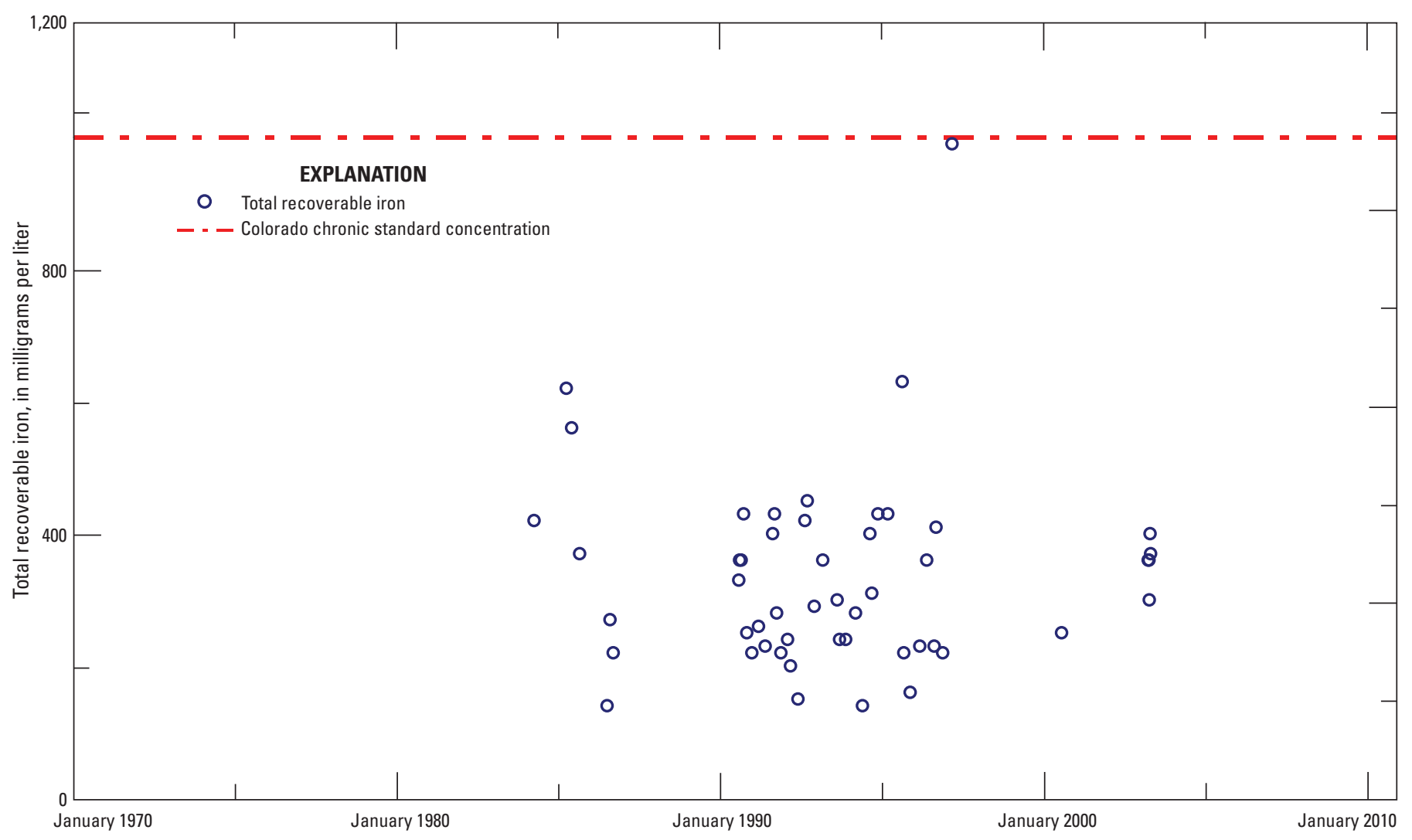

Figure 87. Temporal distribution of total recoverable iron concentrations at site 28, in the Eagle River watershed, Colorado, $1984-2004$.

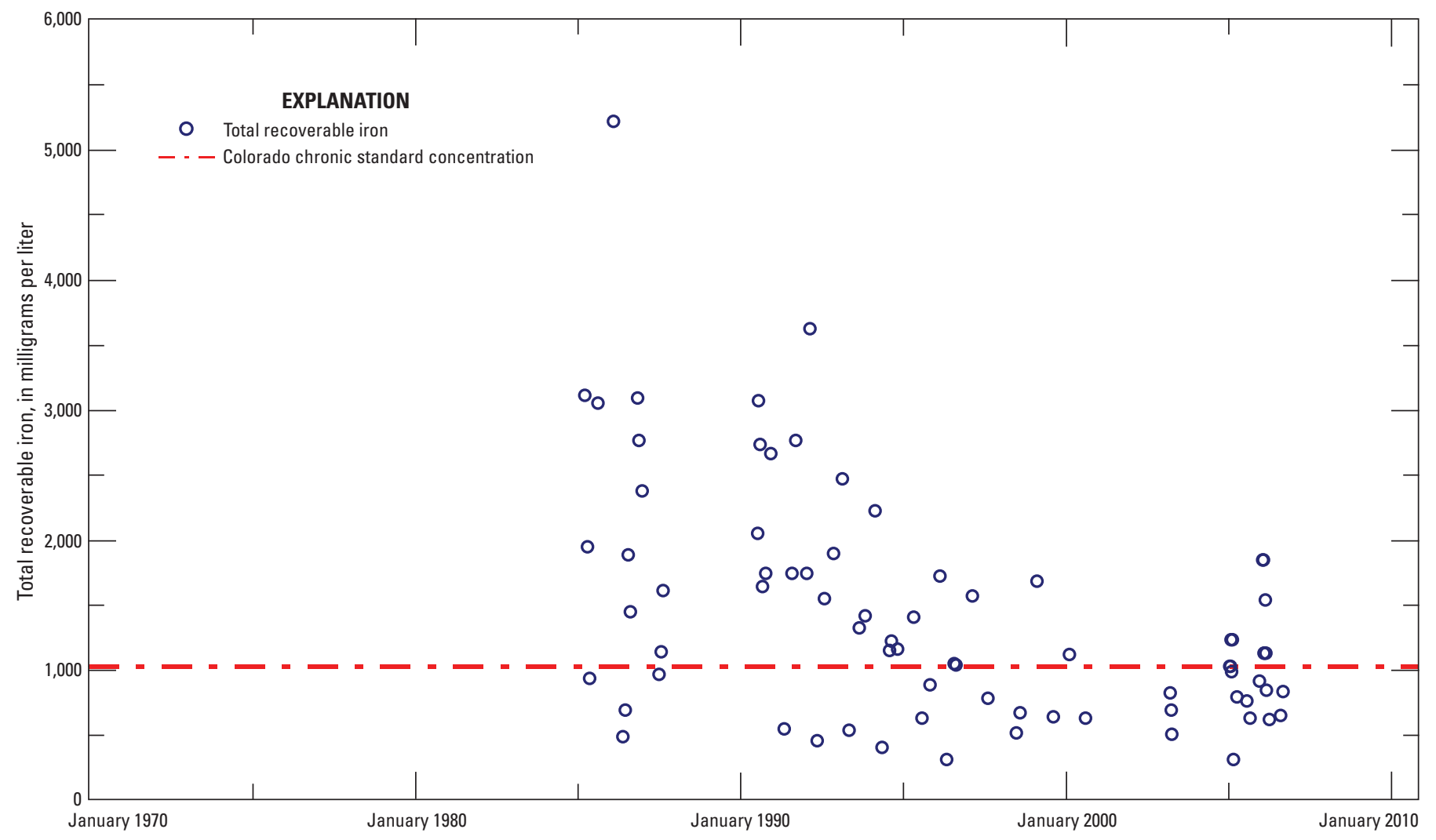

Figure 88. Temporal distribution of total recoverable iron concentrations at site 66, in the Eagle River watershed, Colorado, $1985-2007$. 


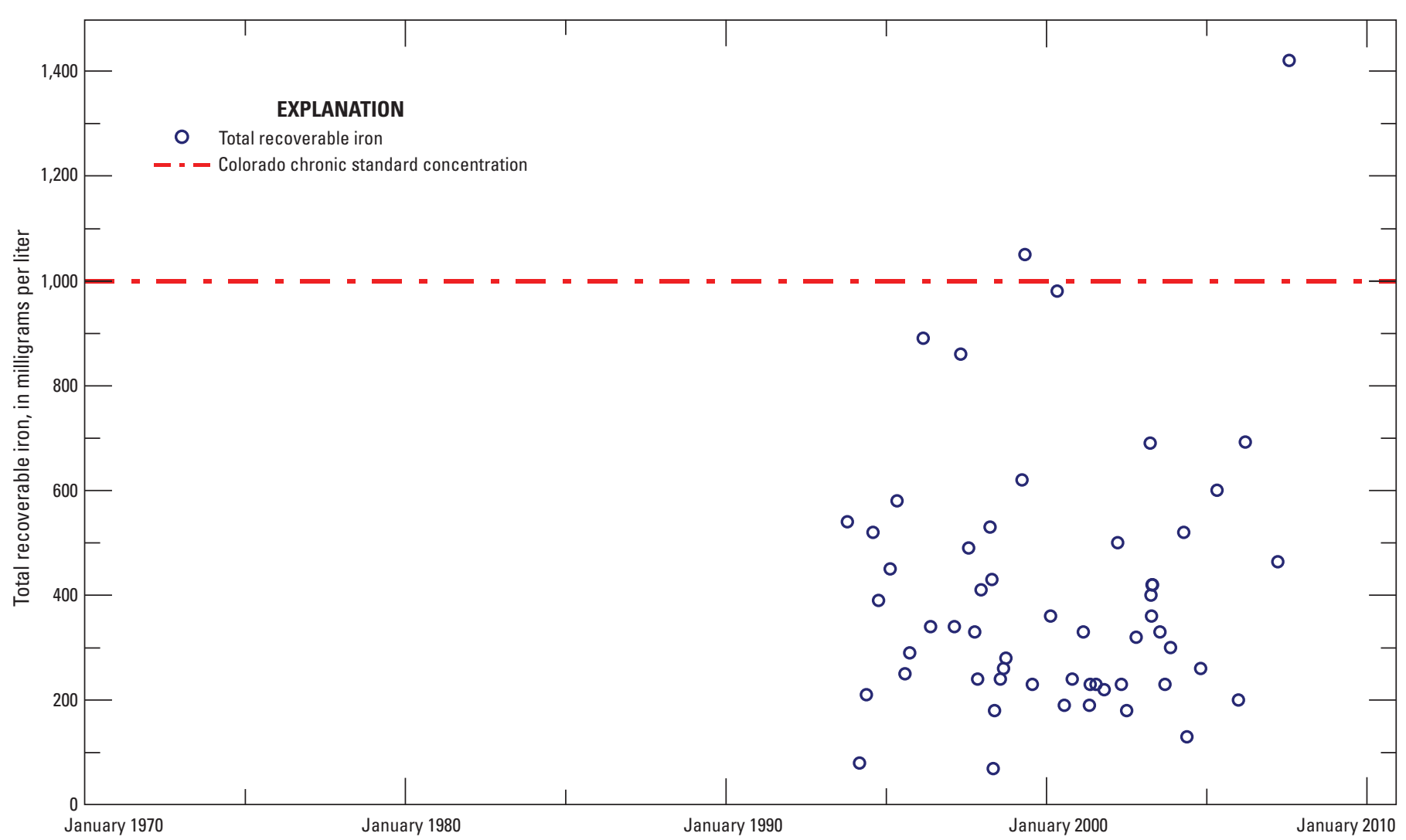

Figure 89. Temporal distribution of total recoverable iron concentrations at site 173, in the Eagle River watershed, Colorado, $1994-2006$.

State standards for zinc are designated by CDPHE. Zinc has a seasonally modified standard within certain reaches of the ERW or a TVS for acute and chronic exposure levels of zinc (Colorado Department of Public Health and Environment, 2008). Two seasons are used to divide the year, March-April and May-February. Reaches 5a, 5b, $5 \mathrm{c}$, and $7 \mathrm{~b}$ have a seasonal designation for the zinc chronic and acute State standards (fig. 12). All other reaches have a TVS that is site specific based on hardness, except for reach 11. The chronic State standards for reach $5 \mathrm{a}$ are $410 \mu \mathrm{g} / \mathrm{L}$ (March-April) and $166 \mu \mathrm{g} / \mathrm{L}$ (May-February); for reach $5 \mathrm{~b}$, $310 \mu \mathrm{g} / \mathrm{L}$ (March-April) and $123 \mu \mathrm{g} / \mathrm{L}$ (May-February); for reach 5c, $257 \mu \mathrm{g} / \mathrm{L}$ (March-April) and a TVS (MayFebruary); and for reach 7b, $193 \mu \mathrm{g} / \mathrm{L}$ (March-April) and $116 \mu \mathrm{g} / \mathrm{L}$ (May-February). Reach 11 has a chronic State standard of 2,000 $\mu \mathrm{g} / \mathrm{L}$ total recoverable. Acute standards for reach 5a are $472 \mu \mathrm{g} / \mathrm{L}$ (March-April) and $178 \mu \mathrm{g} / \mathrm{L}$ (May-February); for reach 5b, $332 \mu \mathrm{g} / \mathrm{L}$ (March-April) and $153 \mu \mathrm{g} / \mathrm{L}$ (May-February); for reach 5c, $275 \mu \mathrm{g} / \mathrm{L}$ (MarchApril) and $127 \mu \mathrm{g} / \mathrm{L}$ (May-February); and for reach 7b, $254 \mu \mathrm{g} / \mathrm{L}$ (March-April) and $120 \mu \mathrm{g} / \mathrm{L}$ (May-February). Reach 11 does not have an acute State standard for zinc.

The chronic State standards for zinc were exceeded less than 20 times over a period of several years for most sites in the ERW; approximately 20 percent (666 comparisons) of the total number of measurements $(3,303)$ exceeded the chronic State standards (table 20).

The acute State standards for zinc were exceeded less than 15 times over a period of several years for most sites in the ERW; approximately 19 percent (628) of the total number of measurements $(3,303)$ exceeded the State standards (table 20). Figures 93-96 show the concentrations for dissolved zinc through time at selected sites in comparison to acute and chronic State standards.

To better represent exceedances of State standards for trace-metals in the ERW as a whole, the combined number of samples with detected exceedances for any of the trace-metals species discussed was made into maps. Maps summarizing the spatial extent of exceedances of acute and chronic State standards for trace metals are presented in figures 97-98.

Evaluation of temporal trends for metals in the ERW includes the evaluation of step trends and monotonic trends. Remediation of the Eagle Mine Superfund site occurred over several years, with the major completion occurring by 1994 (table 2). Comparisons of pre- and post-remediation concentrations for many trace metals shows highly significant decreases in concentrations for cadmium, manganese, and zinc at sites on the Eagle River downstream from the remediation sites and in tributaries affected by remediation activities 


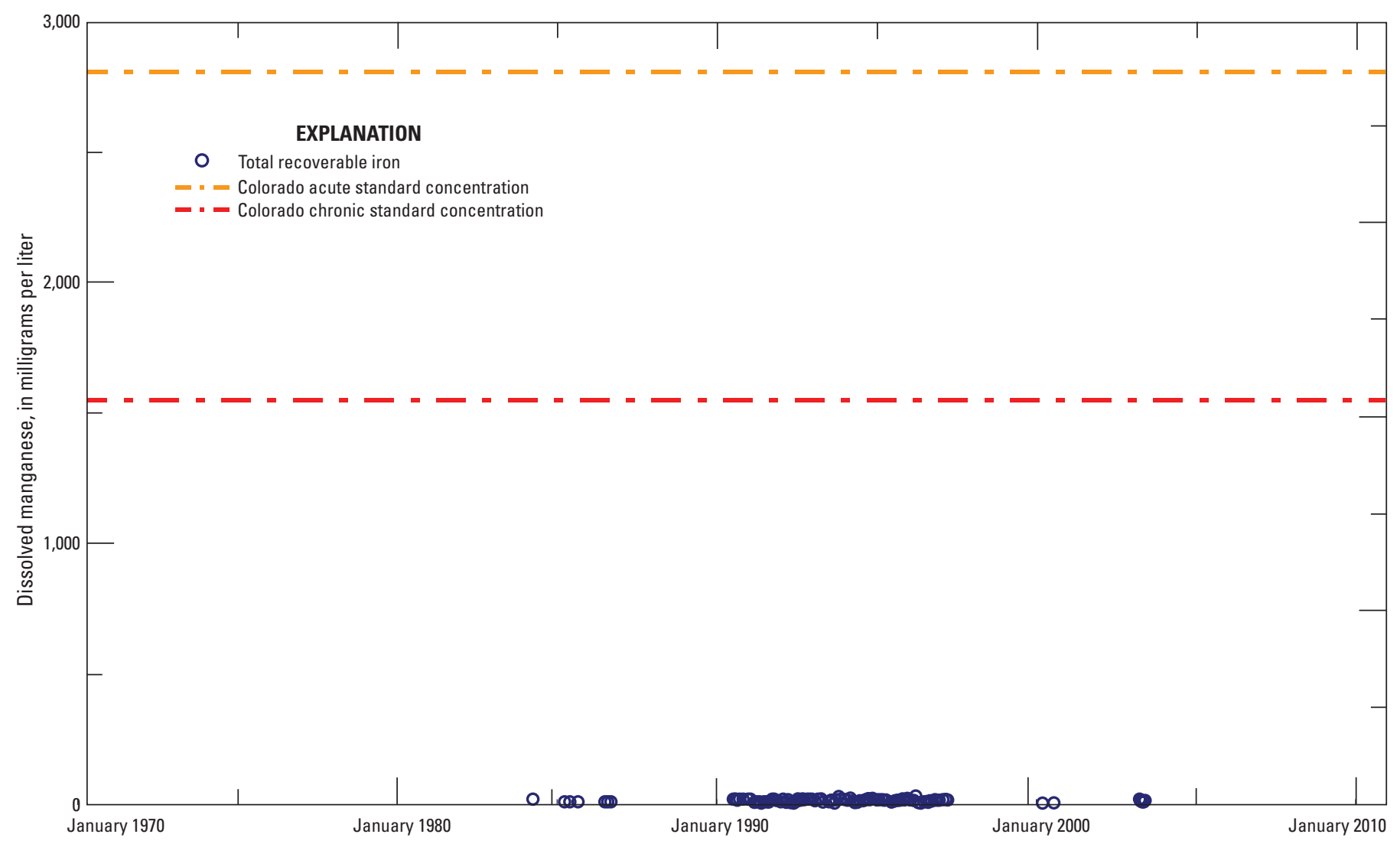

Figure 90. Temporal distribution of dissolved manganese concentrations at site 28, in the Eagle River watershed, Colorado, 1984-2003.

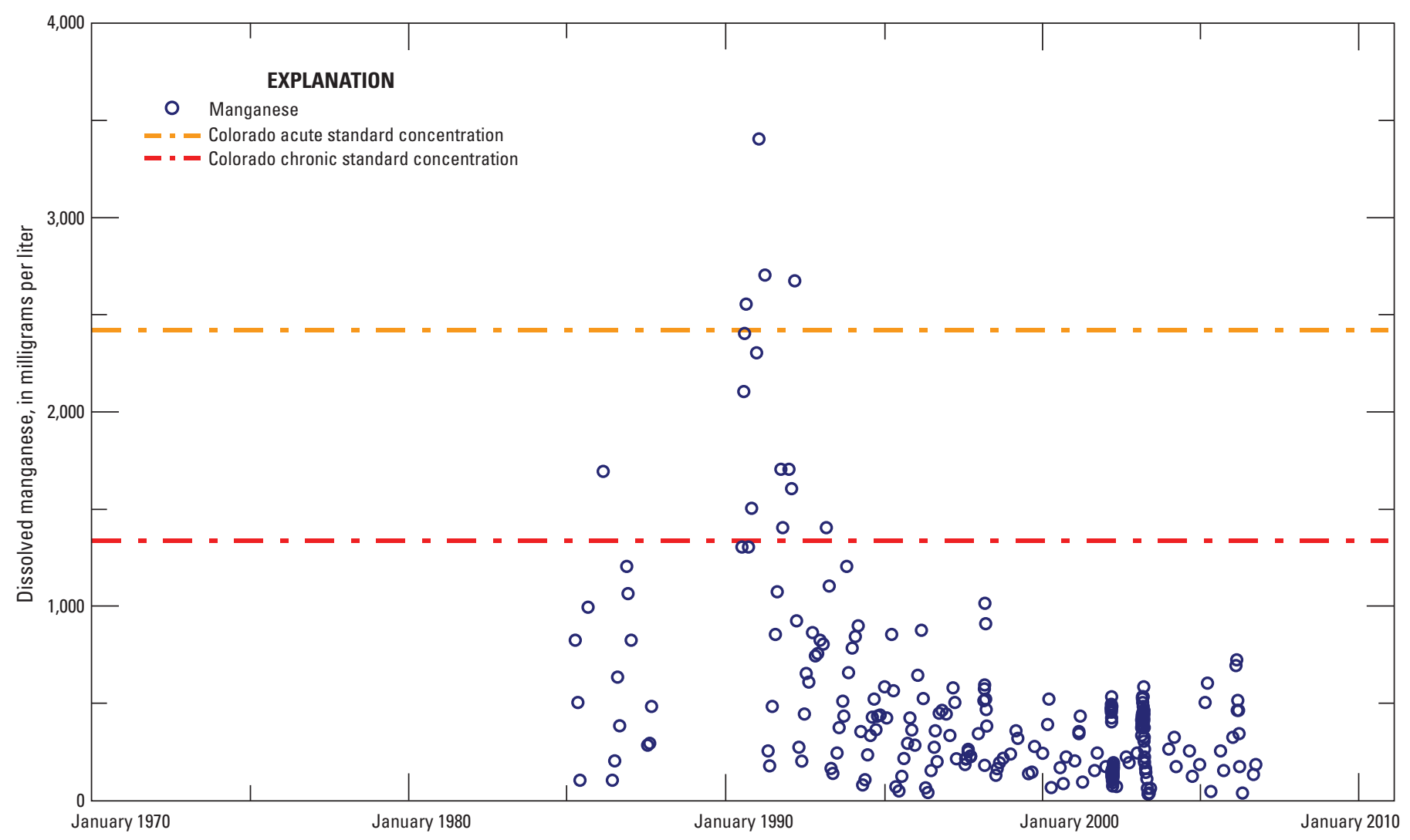

Figure 91. Temporal distribution of dissolved manganese concentrations at site 66, in the Eagle River watershed, Colorado, $1986-2007$. 


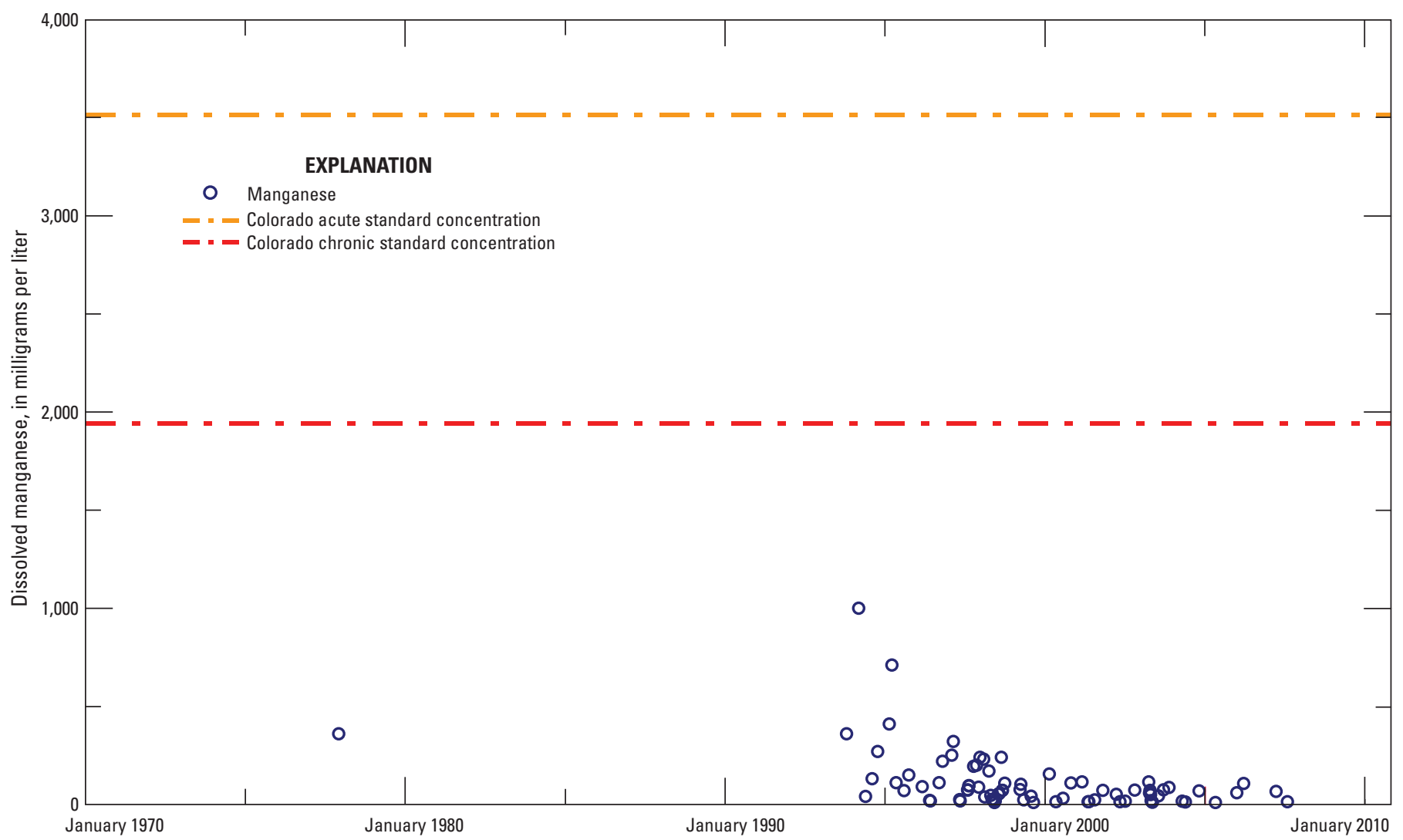

Figure 92. Temporal distribution of dissolved manganese concentrations at site 173, in the Eagle River watershed, Colorado, $1994-2007$.

(table 21). Some sites show order of magnitude reductions in median concentrations between these two periods (table 21). However, iron concentrations do not appear to show the same step-trend reductions between these two periods.

Evaluation of monotonic trends for dissolved trace metals (cadmium, iron, manganese, and zinc) was done for all sites with data that met the criteria set in the "Data Sources, Compilation, Review, and Analysis" section of this report. Findings from the trend analysis are presented in table 22 and figures 64-68. Seasonal Kendall Trend tests were done for the time period 1994-2006 depending on the data available at each site. Numerous downward trends and no upward trends were detected during the study period. Statistically significant trends occur in areas in and downstream from historic mining areas. Spatial continuity of trends within and downstream from the mine area was not always consistent. There were several sites that had no detected trends separating sites with significant downward trends. In general, however, step-trend analysis and temporal-trend analysis provided good evidence that remediation of historic mine areas in the Upper Eagle River subwatershed has lead to observed decreases in many metal concentrations.

\section{Suspended Sediment}

The size and quantity of sediments within streams and the types of sediments transported by a stream are important factors affecting aquatic ecosystems and water management. Sediment can be transported in the water column as suspended sediment or moved along the bed of a stream as bedload. Sediment transport is a combination of the sediment particle size and the transport forces within a stream. Larger particles require larger, continual forces for transport while smaller particles, once in suspension, can remain within a tranquil water column for days before settling out of suspension (Julien, 2010). Varying geologic settings, geologic lithology, and channel form contribute to the sizes and abundance of sediments within a stream (Knighton, 1998).

Sedimentation has been identified by the Colorado Water-Quality Control Commission as a water-quality concern in Black Gore Creek. Black Gore Creek was placed on the Section 303(d) list in 2002, 2004, and 2006. Wynn and others (2001) indicate 4,000 tons of coarse sand and fine gravel are washed into Black Gore Creek each year as a result of traction sanding along I-70. Annual amounts of traction sand applied 


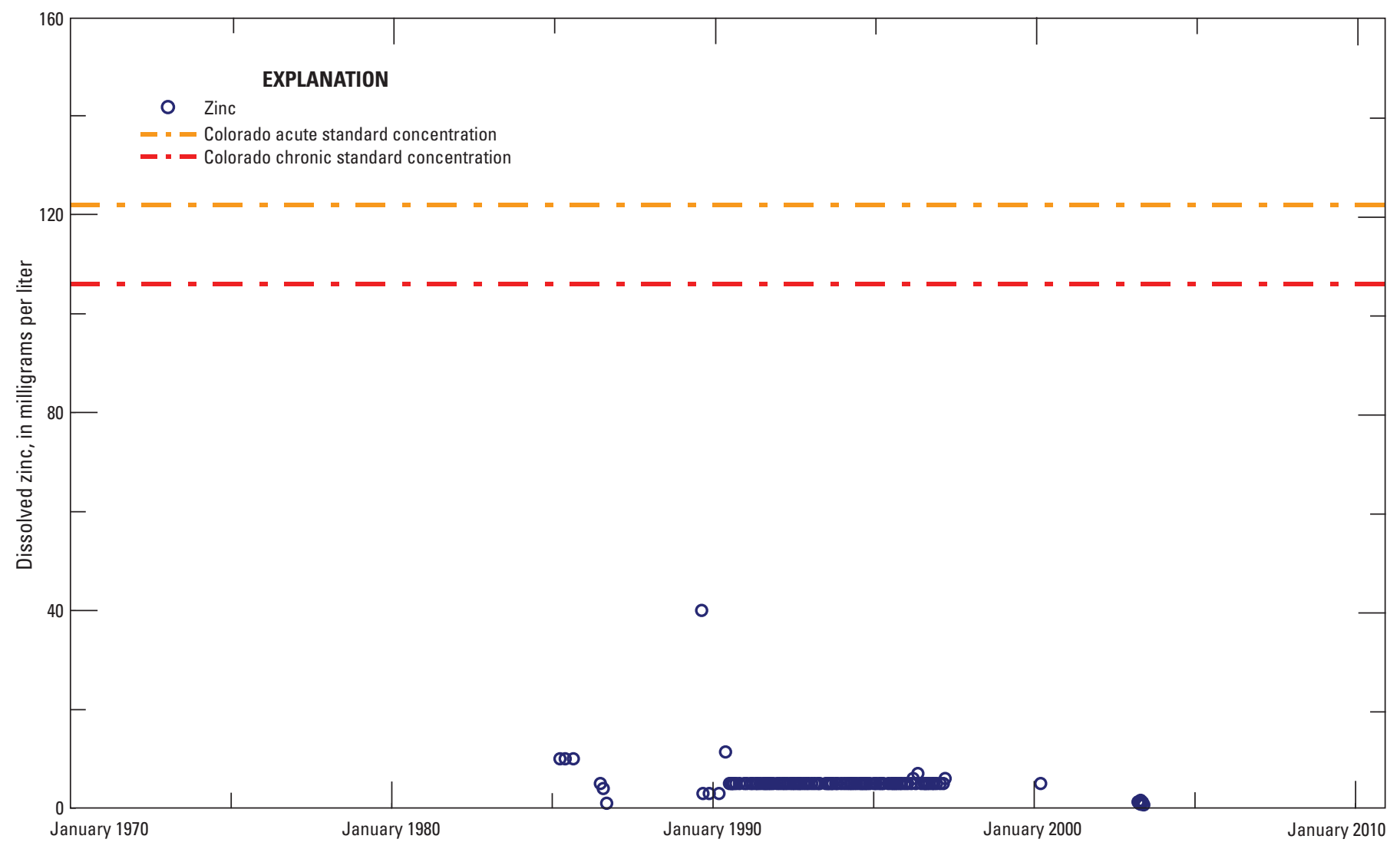

Figure 93. Temporal distribution of dissolved zinc concentrations at site 23, in the Eagle River watershed, Colorado, 1985-2003.

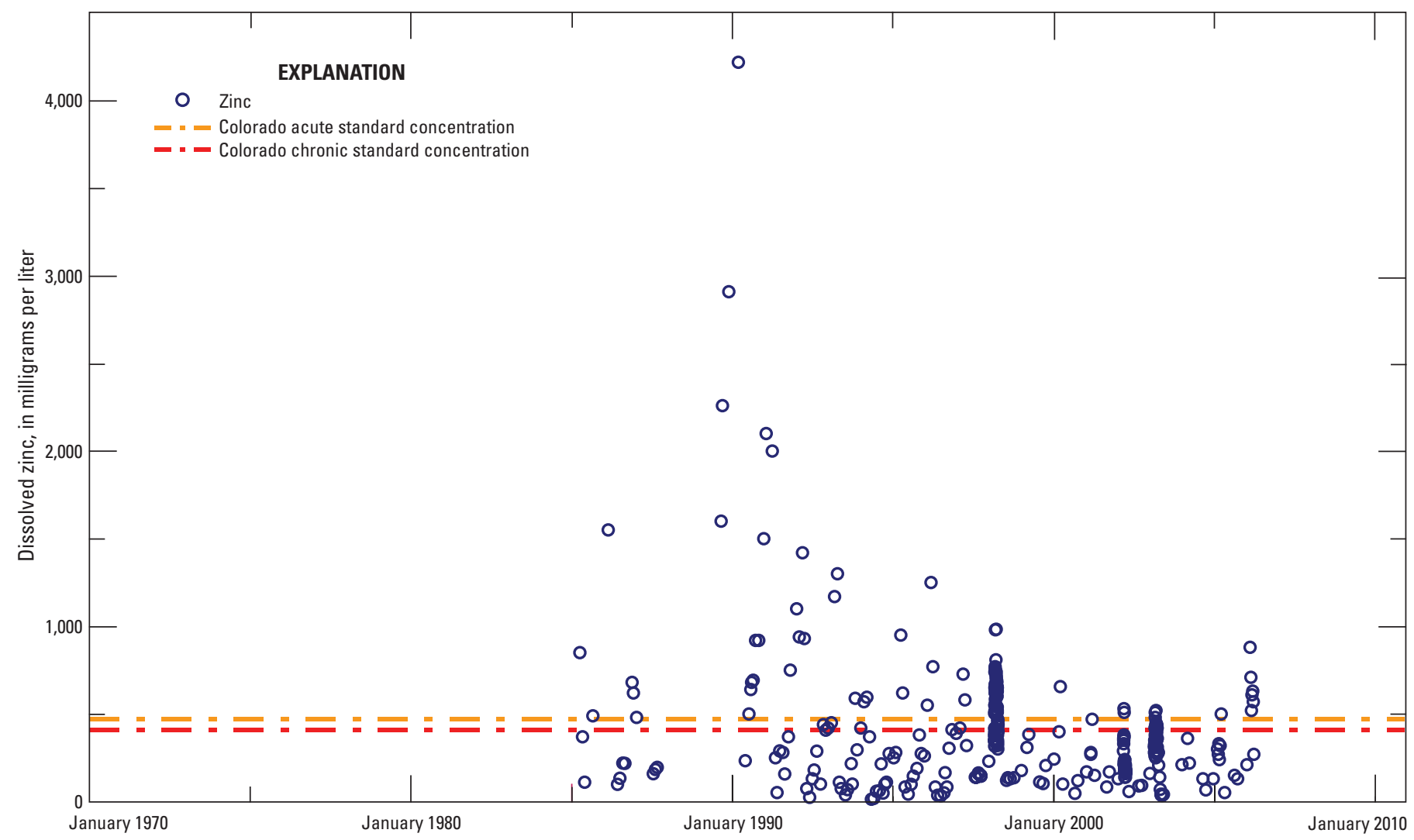

Figure 94. Temporal distribution of dissolved zinc concentrations at site 66, in the Eagle River watershed, Colorado, March-April 1986-2007. 


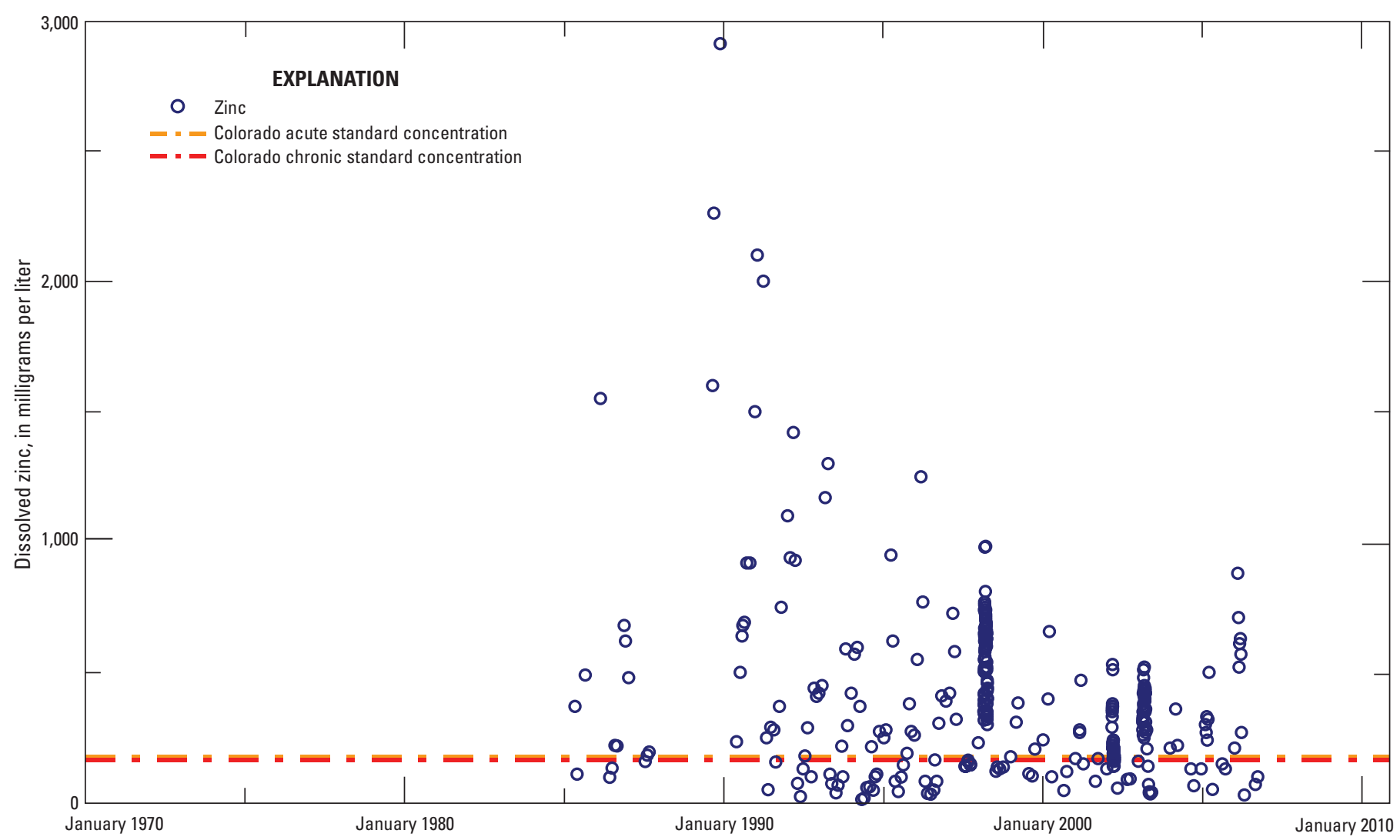

Figure 95. Temporal distribution of dissolved zinc concentrations at site 66, in the Eagle River watershed, Colorado, May-February $1986-2007$.

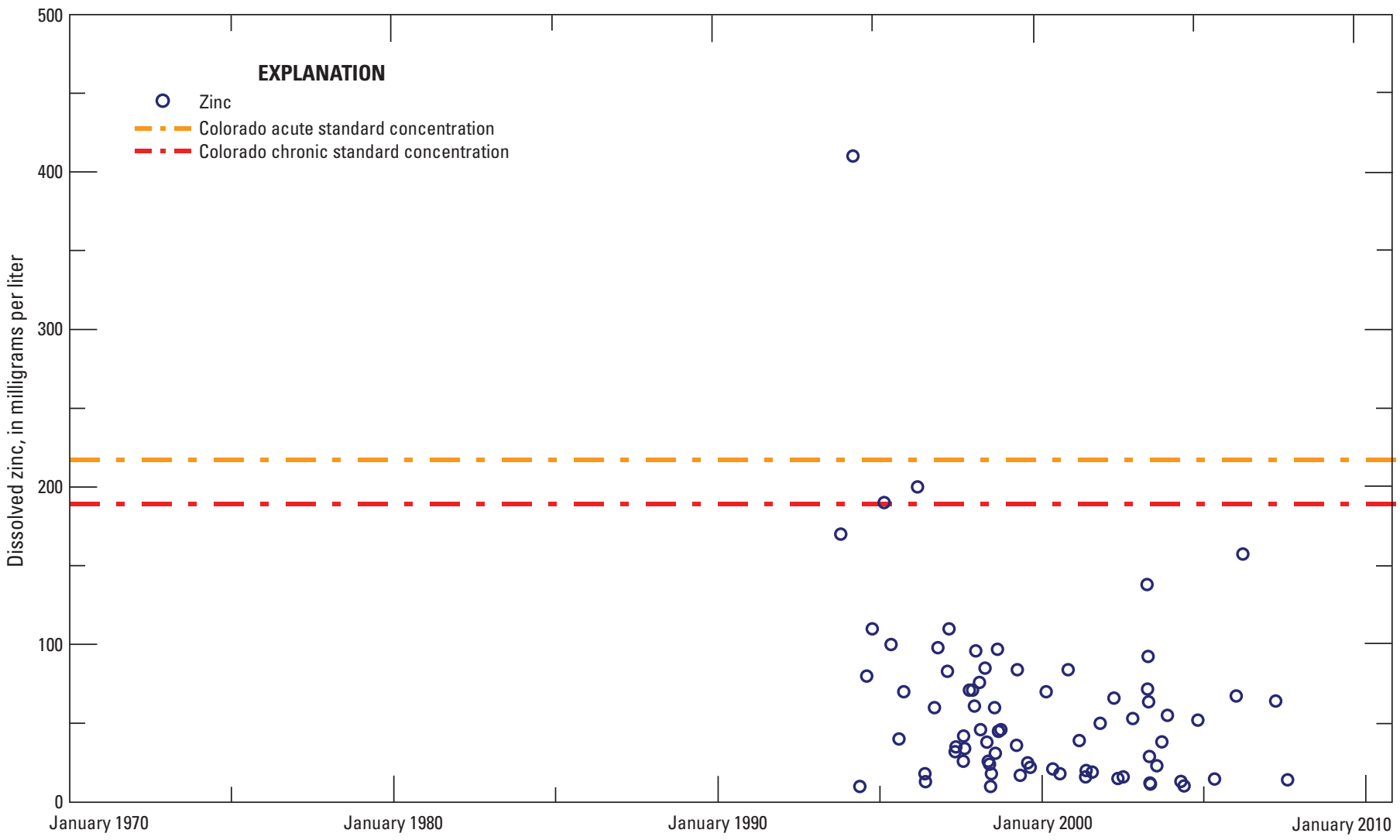

Figure 96. Temporal distribution of dissolved zinc concentrations at site 173, in the Eagle River watershed, 1994-2006. 


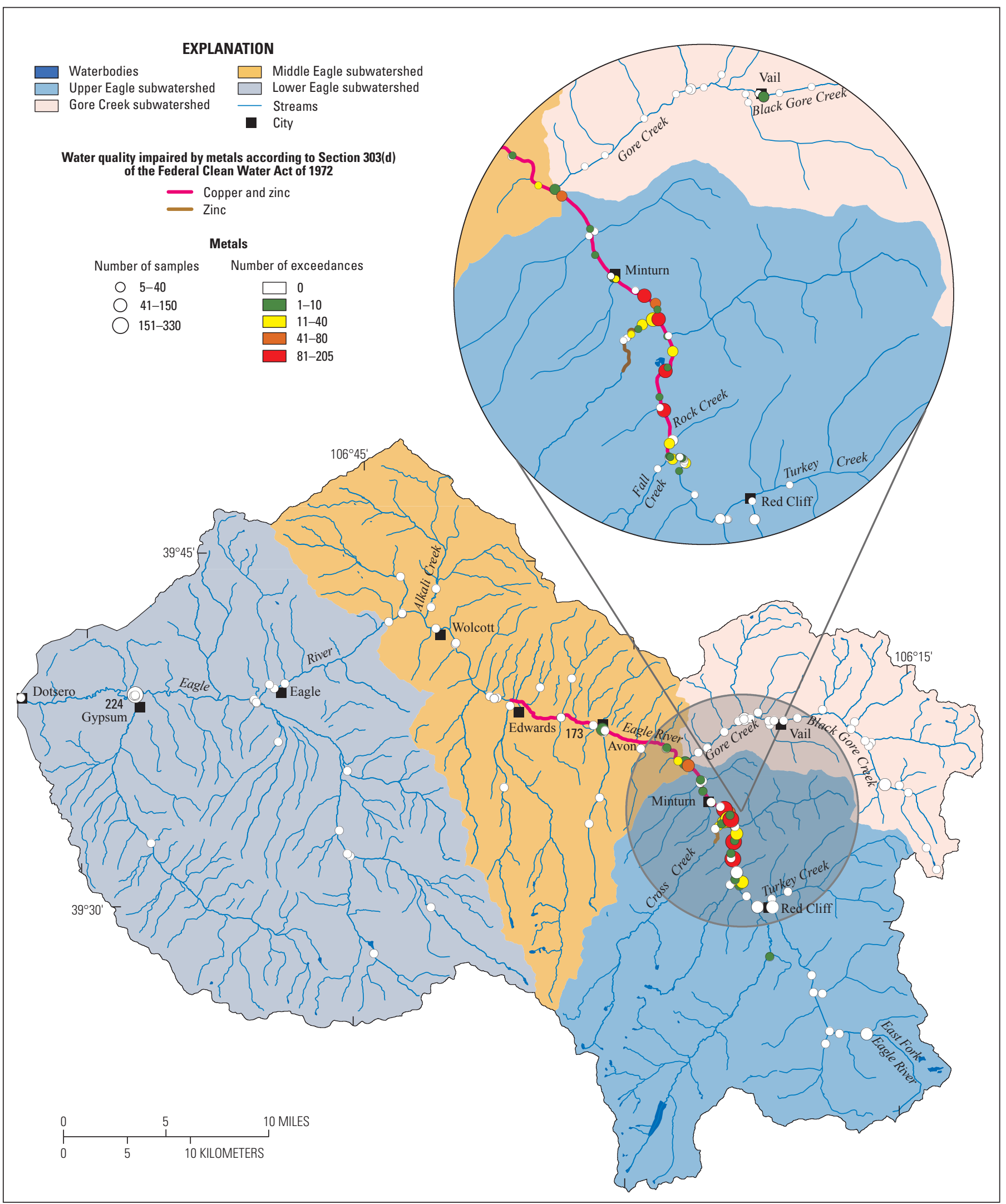

Base from U.S. Geological Survey digital data, 2007,

Universal Transverse Mercator projection

Zone 13

Figure 97. Number of exceedances of the Colorado acute water-quality standard for metals concentrations at selected sites in the Eagle River watershed, Colorado, 1966-2006. 


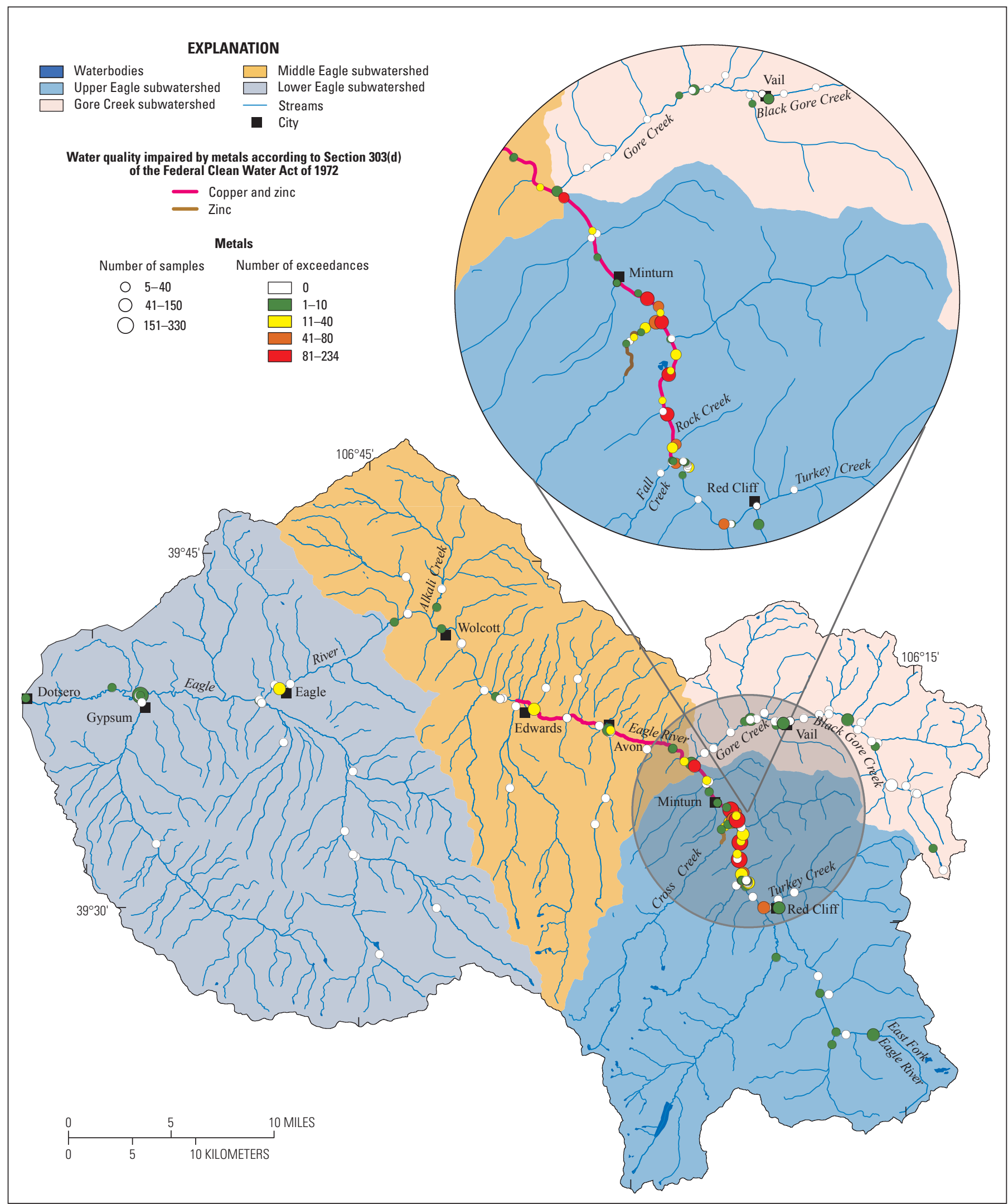

Base from U.S. Geological Survey digital data, 2007,

Universal Transverse Mercator projection

Zone 13

Figure 98. Number of exceedances of the Colorado chronic water-quality standard for metals concentrations at selected sites in the Eagle River watershed, Colorado, 1966-2007. 
Table 21. Summary of step-trends assessment for select sites affected by Eagle Mine Superfund remediation, water years 1972-1991, and 1992-2007.

[No., number; $\mu \mathrm{g} / \mathrm{L}$, micrograms per liter]

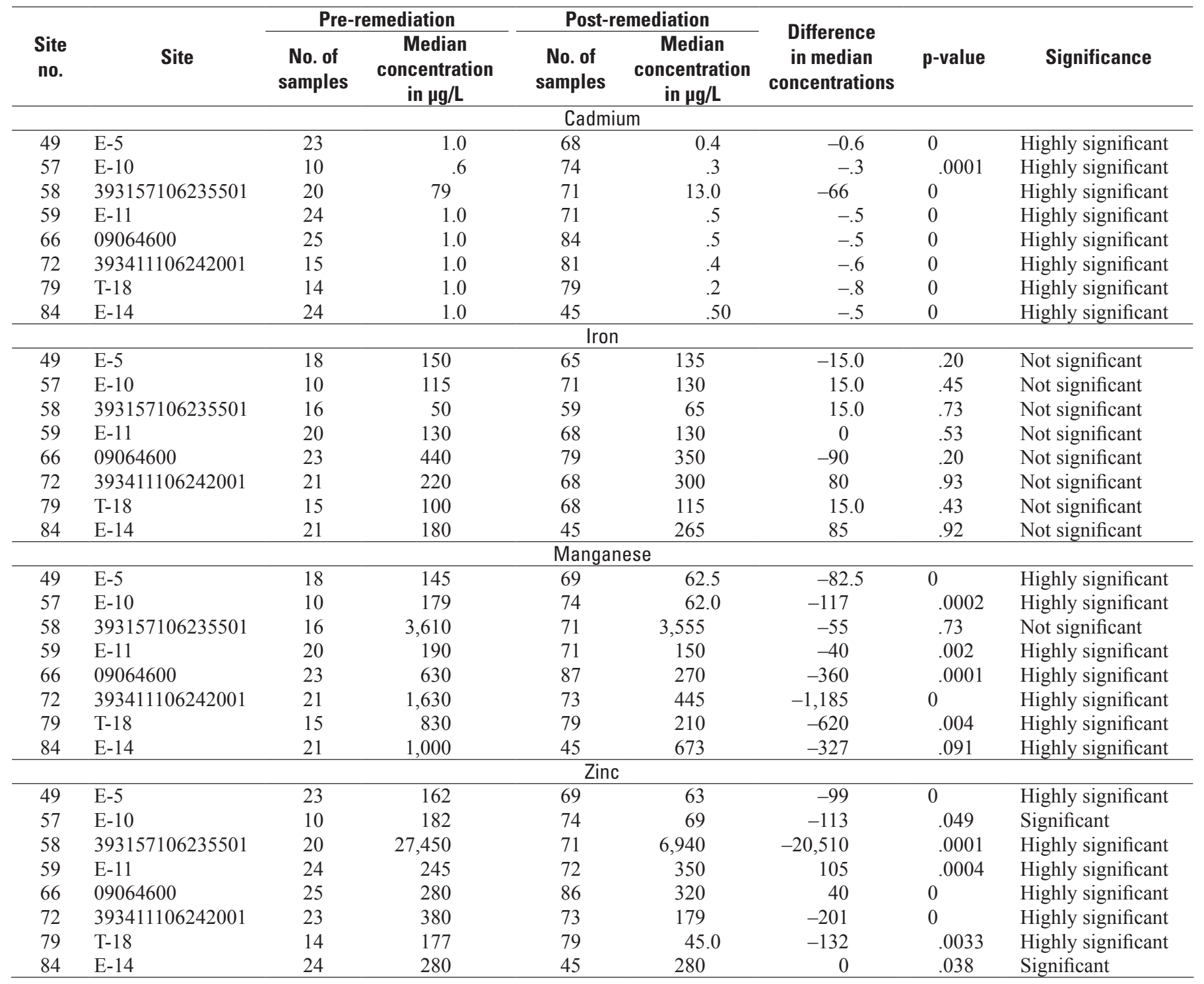


Table 22. Summary of monotonic temporal trends for metals in the Eagle River watershed.

[No., number; $\mu \mathrm{g} / \mathrm{L}$, micrograms per liter; --, no data]

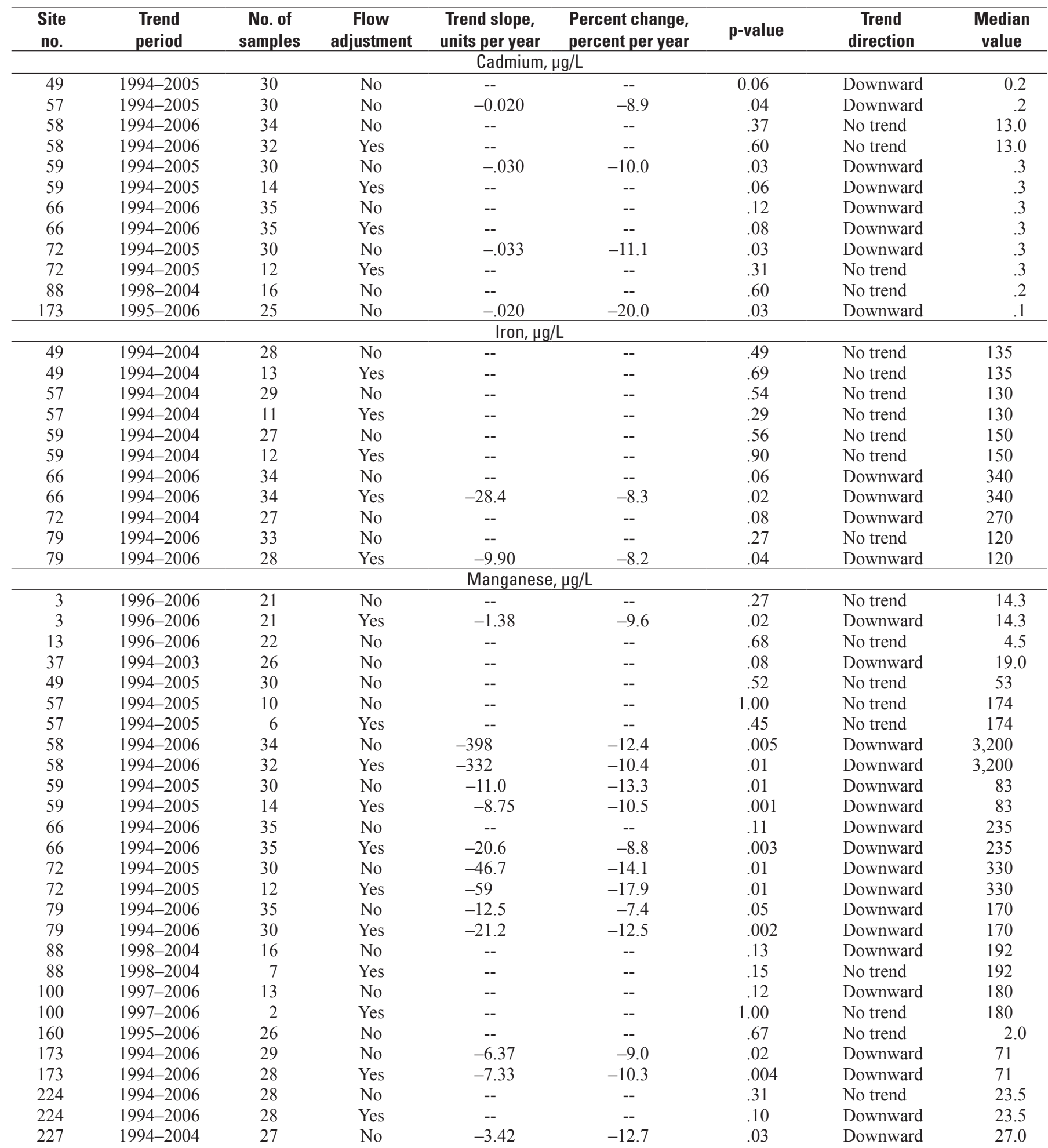


Table 22. Summary of monotonic temporal trends for metals in the Eagle River watershed.-Continued

[No., number; $\mu \mathrm{g} / \mathrm{L}$, micrograms per liter; --, no data]

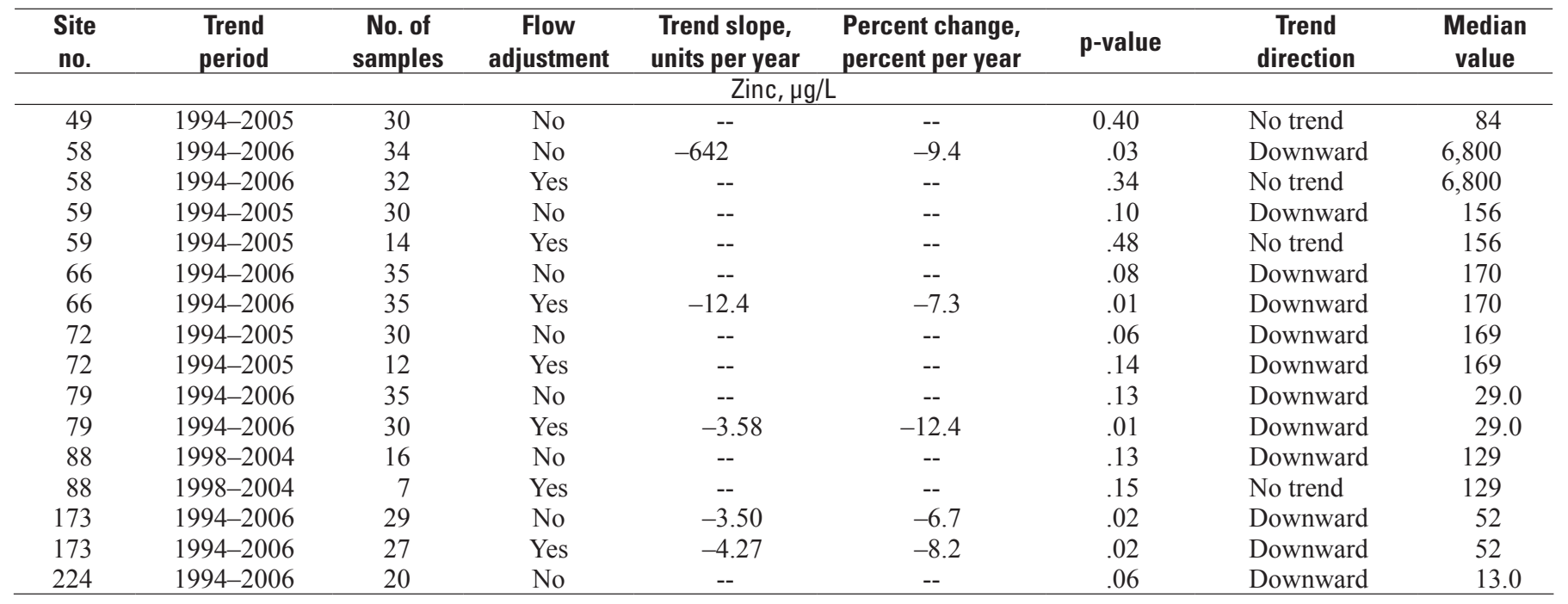

to Black Gore Creek along the I-70 corridor are approximately 500 times the expected natural sediment inputs within the basin of Black Gore Creek (Black Gore Creek Executive Summary, 2006). The accumulation of sediment in streams reduces available spawning habitat for fish species and reduces habitat for macroinvertebrates (Wynn and others, 2001). Increases in the size of sediment particles, along with the total volume of sediments entering a stream, can affect the shape of streams and the size of the bed materials stored within a stream system. Introduction of sediments into a stream from human sources can affect the mobility of sediments within a stream and can lead to aggradation of the streambed. Larger sediment particles require more stream energy to move downstream.

Suspended-sediment concentration has been measured at 72 sites from October 2, 1959, to 2007, and ranged from 0.1 to $1,330 \mathrm{mg} / \mathrm{L}$. The $25 \mathrm{th}, 50 \mathrm{th}$, and 75 th percentiles for the ERW were 2.0, 4.0, and $15.0 \mathrm{mg} / \mathrm{L}$, respectively. Median concentrations for the subwatersheds were $3.0 \mathrm{mg} / \mathrm{L}$ in Gore Creek, $4.0 \mathrm{mg} / \mathrm{L}$ in the Upper Eagle, $7.0 \mathrm{mg} / \mathrm{L}$ in the Middle Eagle, and $23.5 \mathrm{mg} / \mathrm{L}$ in the Lower Eagle (table 8). The spatial pattern shows an increase in suspended-sediment concentration as water moves downstream, with a defined increase in suspended-sediment concentration occurring downstream from Edwards (fig. 99).

Suspended-sediment concentrations indicate variations in seasonal pattern. In Gore Creek, the suspended sediment is highest May-June and lowest December-February (fig. 100). Suspended-sediment concentrations decrease sharply at the end of the runoff period and remain steady through the rest of the year. In the Eagle River, the availability of suspendedsediment data is limited, but available data indicate that suspended sediment is greatest during spring snowmelt runoff.
Evaluation of temporal trends was done for all sites with suspended sediment data; trend analysis was done at the 2 sites that met the criteria set in the "Data Sources, Compilation, Review, and Analysis" section of this report. Findings from the trend analysis are presented in table 9 and figure 99. Seasonal Kendall Trend tests were done for the time period 1995-2007. At sites 224 (Lower Eagle subwatershed) and 160 (Gore Creek subwatershed), no flow-adjusted trends were detected (fig. 99).

\section{Summary}

From the early mining days to the current tourismbased economy, the ERW has undergone a sequence of land-use changes that has affected the hydrology, habitat, and water quality of the area. Although intensive hardrock mining has all but stopped in the watershed, elevated concentrations of metals, such as cadmium, copper, iron, manganese, and zinc, in streams are prevalent as a result of long-standing mining activity in the watershed. A 235-acre site encompassing the Eagle Mine and associated mining wastes between Gilman and Minturn was placed on the Superfund site list in 1986. Remediation of the Eagle Mine site began in 1988 and continues today.

In order to evaluate the water quality, assess effects of growth and associated land-use change, identify temporal and spatial gaps within available water-quality data, and evaluate spatial and temporal trends in water quality, an analysis of historical data was done. In 2000, the USGS, in cooperation with the Colorado River Water Conservation District, Eagle 


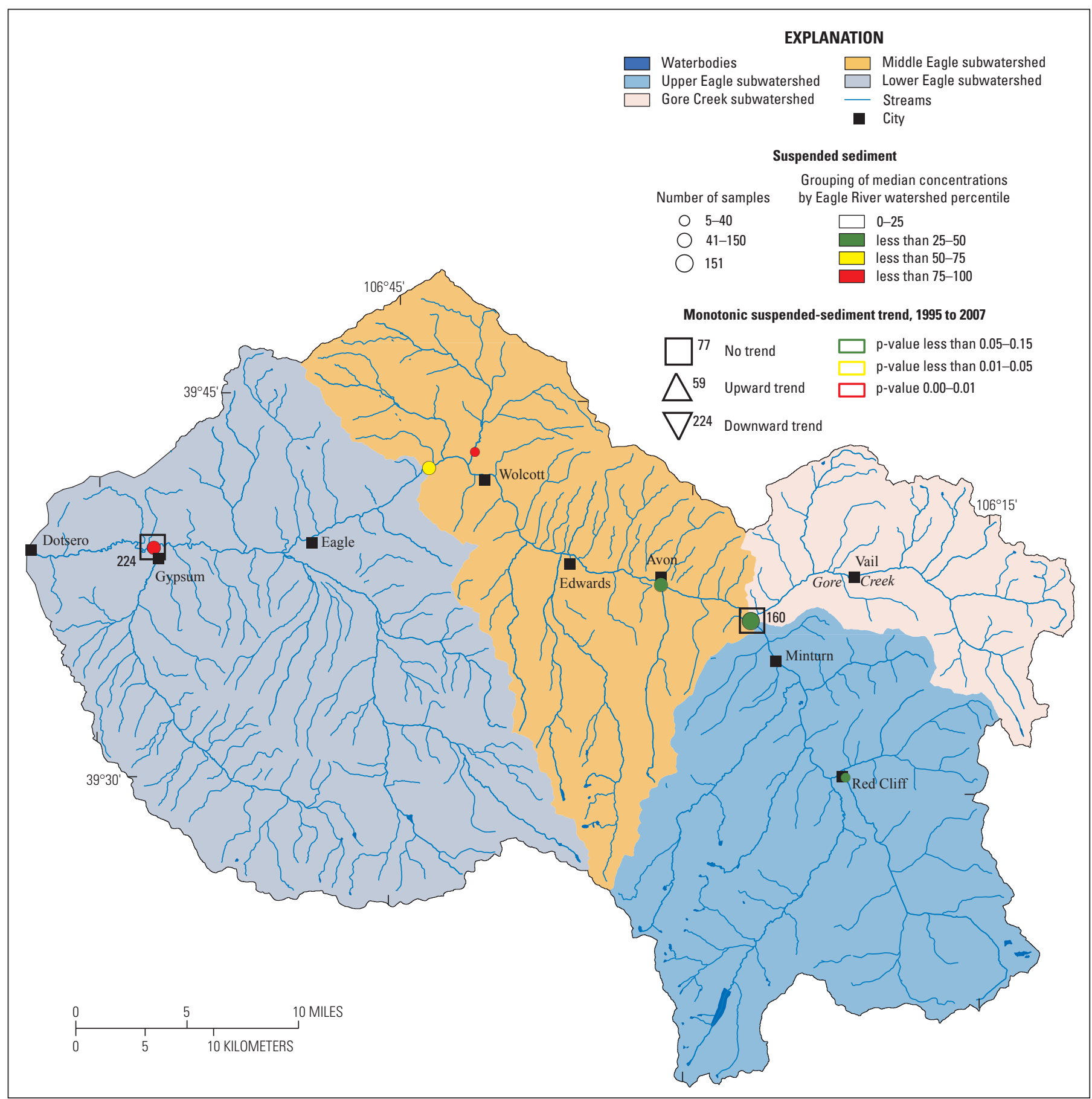

Base from U.S. Geological Survey digital data, 2007,

Universal Transverse Mercator projection

Zone 13

Figure 99. Spatial distribution of median-concentration percentiles and monotonic suspended-sediment concentration trends at selected sites in the Eagle River watershed, Colorado, 1995-2007. 


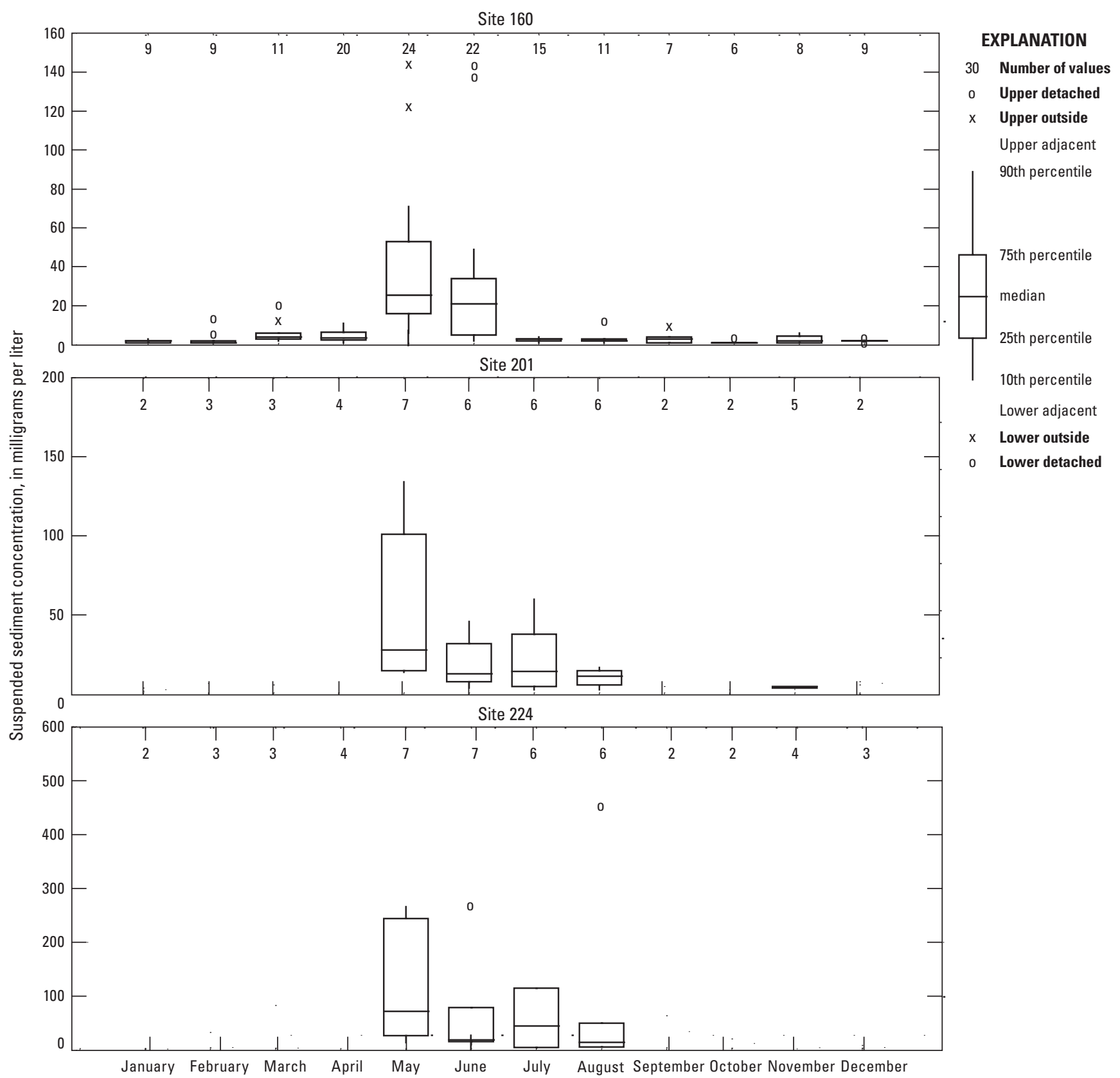

Figure 100. Seasonal pattern in suspended-sediment concentration at selected sites in the Eagle River watershed, Colorado, $1947-2007$. 
County, Eagle River Water and Sanitation District, Upper Eagle Regional Water Authority, Colorado Department of Transportation, City of Aurora, Town of Eagle, Town of Gypsum, Town of Minturn, Town of Vail, Vail Resorts, Colorado Springs Utilities, and Denver Water initiated a retrospective analysis of surface-water quantity and quality in the ERW. Data were gathered and reported from the U.S. Geological Survey, the U.S. Environmental Protection Agency, U.S. Forest Service, Colorado Department of Natural Resources, Colorado Department of Public Health and Environment, Colorado Division of Water Resources, Colorado Department of Transportation, Colorado Division of Wildlife (River Watch), Eagle Mine Superfund Site Assessment, Colorado Springs Utilities, Eagle River Water and Sanitation District, Grand River Consulting, and Advanced Sciences Incorporated.

The Eagle River drains approximately 963 square miles $\left(\mathrm{mi}^{2}\right)$ west of the Continental Divide in central Colorado before flowing into the Colorado River. Located in central Colorado west of Vail Pass, the Eagle River watershed (ERW) is almost entirely contained within Eagle County. The watershed extends from the eastern boundary near Vail Pass to the western boundary near Dotsero.

For selected water-quality properties and constituents in the watershed, this report: (1) characterizes available water-quantity and water-quality data, (2) identifies spatial and seasonal variability in water quantity and water quality, (3) provides comparisons to Federal and State water-quality standards or recommendations, (4) characterizes temporal changes in water quality, and (5) where possible, identifies potential causes of these changes. The study area was divided into four subwatersheds for this analysis: (1) Upper Eagle, Eagle River including all tributaries upstream from Gore Creek; (2) Gore Creek, including all tributaries upstream from the Eagle River: (3) Middle Eagle, Eagle River including all tributaries downstream from Gore Creek and upstream of the USGS streamflow-gaging station at Wolcott; (4) Lower Eagle, Eagle River including all tributaries downstream from the USGS streamflow-gaging station at Wolcott and upstream from the Colorado River.

Available surface-water quantity data were obtained from the USGS National Water Information System and the City of Colorado Springs. Available surface-water quality data were obtained from local, State, and Federal agencies and entered into a relational database to assist in the analysis of waterquality conditions in the ERW 1947-2007. The data used in the analysis described in this report are available in a web-based repository at http://rmgsc.cr.usgs.gov/cwqdr/Eagle/index.shtml.

An inclusive approach was taken to expand the number of samples and sites available for analysis in order to provide greater spatial and temporal coverage of water-quality conditions in the watershed. A large portion of the data has limited metadata and (or) quality-assurance data. Therefore, it is possible that some data may contain errors that were not detected during the water-quality data review.

Streamflow conditions of the Eagle River follow a consistent seasonal pattern. Low-flow conditions (base flow) typically occur in October-March, with the start of the snowmelt-runoff generally beginning in late March and early April. As the snowpack begins to melt, streamflows begin to rise and increase to peak streamflow conditions in late May and early June. As available snowpack levels are depleted in late spring and early summer streamflow levels return to the base-flow conditions. Occasional increases in flow during the summer and fall months can occur because of precipitation events within the ERW.

Many sites within the ERW show patterns of wet and dry years. The mid 1980s and 1990s are two wet periods within the last several decades, separated by average to dry periods in 1990 and 2000. The wettest and driest years on record at most sites are 1984 and 2002, respectively.

Surface-water-quality data from 293 sites and 12 different source agencies were complied into 191 unique sites located on streams and rivers in the ERW. Nearly 39 percent (74 sites) of the unique sites had fewer than 5 samples. In comparison, 23 percent (44 sites) of the sites have more than 100 samples. Physical properties were the most abundant type of samples collected, with major ions, nutrients, and trace elements also commonly collected. As mentioned in "Data Review and Analysis," where adequate data existed, determination of summary statistics, comparisons to State standards and Federal recommendations, and trend analysis were done. Analysis results were separated into different sections of the report based on constituent groupings.

Field properties analyzed for in this study included specific conductance, $\mathrm{pH}$, water temperature, dissolved oxygen, and hardness.

Specific conductance is proportional to the dissolved-solids concentration within the stream and has the ability to conduct an electric current. The spatial pattern shows an increase in specific conductance as water moves downstream. There is a significant downward trend at site 59 (Upper Eagle subwatershed).

The $\mathrm{pH}$ is a measure of how acidic or basic a solution is. In areas heavily affected by mining the median concentrations are lower, with seasonally lower $\mathrm{pH}$ during February and March. In other areas within the basin, $\mathrm{pH}$ tends to be lowest during peak streamflows (May and June) and highest during late fall and early spring. The spatial pattern shows increase in $\mathrm{pH}$ as water moves downstream. Approximately 2 percent of measurements in the ERW are outside the State's range of acceptable values. There is a weakly significant upward trend in $\mathrm{pH}$ at site 66 (Upper Eagle subwatershed); a highly significant upward trend at site 173 (Middle Eagle subwatershed); and a weakly significant upward trend at site 224 (Lower Eagle subwatershed).

Water temperatures in the ERW generally increase in the downstream direction with the highest median temperature observed near Gypsum. Water temperatures tend to be coldest December-February and warmest July-August following ambient air-temperature patterns. Less than 1 percent of the total numbers of measurements exceed the State standard for chronic water temperature. There are significant upward trends at site 13 (Upper Eagle subwatershed) and site 160 (Gore Creek subwatershed). 
Dissolved-oxygen concentrations are inversely related to water temperatures; as temperatures increase the capacity of water to hold a given quantity of dissolved oxygen is reduced. The spatial pattern for dissolved oxygen is variable within the ERW. Concentrations tend to be lowest from July-September and highest from November-March. Approximately 4 percent of measurements exceed the State standards for dissolved oxygen. There is a highly significant upward trend (p-value less than 0.01) at site 173 (Middle Eagle subwatershed).

Hardness can have many definitions, in this analysis it will be interpreted as a measure of the calcium and magnesium abundance. The spatial pattern for hardness shows increases in concentration as water moves downstream in the ERW. Concentrations tend to be lowest May-June and highest JanuaryMarch, which is the inverse of patterns in streamflow. There is a significant downward trend in hardness at site 224 (Lower Eagle subwatershed).

Dissolved solids are calculated from (or a measures of) ions within a water-quality sample, and can be thought of as the dissolved salts that are present. The major ions dissolved in most natural waters typically include calcium, magnesium, sodium, potassium, alkalinity, sulfate, chloride, fluoride, and silica. The spatial pattern for dissolved solids shows increasing concentrations from upstream to downstream with a defined increase occurring near the town of Wolcott and likely resulted from salt-bearing geologic formations (such as the Eagle Valley Evaporite) within the area. Concentrations tend to be lowest May-June and highest October-March, which is the inverse of patterns in streamflow. There is a significant upward trend at site 160 (Gore Creek subwatershed), a weakly significant downward trend at site 224 (Lower Eagle subwatershed). Less than 1 percent of the measurements exceeded the State standards for chloride.

The two sites with detected trends in dissolved solids (site 160 and site 224) were evaluated for trends in calcium, magnesium, sodium, potassium, alkalinity, sulfate, chloride, and silica. At site 160 (Gore Creek subwatershed), highly significant upward trends were found for sodium, potassium, and chloride; with a significant upward trend in magnesium; and a weakly significant trend in calcium. At site 224 (Lower Eagle subwatershed), significant downward trends were detected in calcium, magnesium, sulfate, and silica; and weakly significant upward trends were observed in sodium, potassium, and chloride. At site 160 (Gore Creek subwatershed) relative abundances of the ions indicates that chloride salts likely are the source for the detected upward trends. A potential source for the observed chloride salts may be the chemical anti-icing and deicing products used during winter road maintenance in municipal areas and on Interstate-70.

Nitrogen and phosphorus are two nutrients that can be present in varying chemical forms within the watershed. The spatial pattern of nutrients shows lower concentrations on many tributaries and on the Eagle River upstream from the town of Red Cliff with increases in nutrients downstream of major urban areas. Seasonal variations show that for many nutrient species, concentrations tend to be lowest May-June and highest
January-March. At site 224 (Lower Eagle subwatershed), total phosphorus concentrations are elevated January-May and July-September with concentrations highest in April. These higher concentrations may be related to sediment transported by snowmelt runoff and rainfall. From less than 1 percent to 9 percent of nitrogen nutrients measurements exceeded the State standards. Approximately 24 percent of phosphors nutrients measurements exceeded Federal recommendations. There is a significant upward trend in nitrate at site 224 (Lower Eagle subwatershed); a weakly significant upward trend in nitrite at site 160 (Gore Creek subwatershed); and a significant upward trend in total phosphorus and a weakly significant upward trend in orthophosphate at site 224 (Lower Eagle subwatershed). A positive correlation was observed between concentrations of suspended sediment and total phosphorus.

Trace elements often are defined as those elements that generally occur in concentrations less than 1,000 micrograms per liter. The discussion of trace elements has been separated into two groups: trace elements (arsenic, selenium, and uranium) and trace metals (aluminum, cadmium, copper, iron, manganese, and zinc).

The spatial pattern showed an increase in trace-metal concentrations downstream from historic mining areas and decreased concentrations or concentrations below reporting limits in Gore Creek and other tributaries within the ERW. Concentrations tend to be lowest May-June and highest November-April for many metals. Concentration in early spring (February-April) can be substantially higher as the leading edge of snowmelt waters interacts with mine shafts and (or) adits, mineralized rock units, and other areas affected by processed mine wastes and contaminated soils. The seasonal pattern of dissolved iron at site 224 (Lower Eagle subwatershed) is different from other metals in that dissolved iron concentrations are relatively stable for most of the year with higher concentrations April-July. The increased concentrations of dissolved iron may best be explained by the colloidal behavior of iron. Trace-element exceedances of State standards are generally less than 1 percent of the measurements. Trace-metal exceedances of State standards varied by metal but were generally more numerous than the exceedances for the trace elements. Step-trend analysis of trace-metal concentrations at some sites show order of magnitude reductions in median concentrations following major completion of remediation in the Eagle Mine Superfund Site. In general, step-trend analysis and monotonic-trend analysis provided good evidence that remediation of historic mine areas in the upper Eagle River subwatershed has lead to observed decreases in many metal concentrations.

Sediment can be transported in the water column as suspended sediment or moved along the bed of a stream as bedload. Sedimentation has been identified by the Colorado Water-Quality Control Commission as a water-quality concern in Black Gore Creek and was placed on the Section 303(d) list in 2002, 2004, and 2006. Traction sanding of Interstate-70 in the Gore Creek subwatershed has been identified as a source of sands to Black Gore Creek. The spatial pattern from available sampling locations shows an 
increase in suspended-sediment concentration as water moves downstream in the ERW, with a defined increase in suspendedsediment concentration occurring downstream from the town of Edwards. In the ERW available data indicate that suspended sediment is greatest during spring snowmelt-runoff (MayJune). No trends in suspended sediment were detected.

\section{Acknowledgments}

The authors wish to express thanks and appreciation to the many agencies that provided data for this report. A special thanks to Ray Merry with Eagle County and Caroline Byus with Eagle River Water and Sanitation for their assistance and continued support in the watershed. The authors express gratitude to USGS employees Jude Thomas, Jean Dupree, and Bob Zuellig for their contributions. Acknowledgments are extended to Jason Gurdak, Liza Miller, and Dave Kanzer for their technical review of the manuscript.

\section{References Cited}

Allan, J.D., 1996, Stream ecology structure and function of running waters: London, Chapman \& Hall, 388 p.

Black Gore Creek Executive Summary, 2006, Black Gore Creek total maximum daily load: Sediment Source Monitoring Report 2006, 31 p.

Bledsoe, B., Meyer, J., Holburn, E., Cuhaciyan, C., Earsom, S., and Snyder, B., 2005, Eagle River inventory and assessment: Final report to the Eagle River Watershed Council, Colorado Department of Public Health and Environment, and Great Outdoors Colorado, 550 p., and appendixes.

Butler, D.L., 2001, Effects of piping irrigation laterals on selenium and salt loads, Montrose Arroyo Basin, western Colorado: U.S. Geological Survey Water-Resources Investigations Report, 01-4204, 14 p.

Brown, T.L., LeMay, H.E., Bursten, B.E., 2006, Chemistrythe central science: New Jersey, Prentice Hall, 1128 p.

Center for Disease Control and Prevention, 2011, Harmful algal blooms (HABs): facts about cyanobacteria and cyanobacterial harmful algal blooms, accessed March 1, 2011 at http://www.cdc.gov/hab/cyanobacteria/facts.htm\#cyano.

Church, S.E., Kimball, B.A., Fey, D.L., Ferderer, D.A., Yager, T.J., and Vaughn, R.B., 1997, Source, transport, and partitioning of metals between water, colloids, and bed sediments of the Animas River, Colorado: U.S. Geological Survey Open-File Report 97-151, 135 p.

Colorado Department of Public Health and Environment, 2009, Special topics: Section 303(d) Total maximum daily load program, accessed March 2, 2009, at http://www.cdphe. state.co.us/op/wqcc/SpecialTopics/SpecialTopics.html.
Colorado Department of Public Health and Environment, 2002, Colorado mixing zone implementation guidance, 64 p. (also available at $h t t p: / / w w w . c d p h e . s t a t e . c o . u s / w q /$ permitsunit/POLICYGUIDANCEFACTSHEETS/ PolicyandGuidance/MixingZone.PDF.)

Colorado Department of Public Health and Environment, 2000, Section 7, Population: Demography section of the Colorado Division of local government, accessed April 8, 2009, at http://www.cdphe.state.co.us/hs/population.pdf.

Colorado Department of Public Health and Environment, 2007, Regulation no. 33, classifications and numeric standards for Upper Colorado River Eagle River Watershed and North Platte River (Planning Region 12) (5 CCR 1002-33): Colorado Department of Public Health and the Environment, Water Quality Control Commission, accessed December 14, 2007) http://www.cdphe.state. co.us/regulations/wqccregs/index.html.

Colorado Department of Public Health and Environment and U.S. Environmental Protection Agency, 2008, Eagle Mine Superfund Site, Eagle County, Colorado: Third Five-Year Review Report, 88 p.

Colorado Department of Public Health and Environment and U.S. Environmental Protection Agency, 2005, Eagle Mine Superfund Site, Eagle County, Colorado: Second Five-Year Review Report, $97 \mathrm{p}$.

Colorado Department of Public Health and Environment and U.S. Environmental Protection Agency, 2000, Eagle Mine Superfund Site, Eagle County, Colorado: Five-Year Review Report, 113 p.

Colorado Department of Transportation, 2008, De-icer factsheet, (also available at $h$ ttp://ext.dot.state.co.us/staging/ librarysection/Brochures/DeicerFactSheet.pdf/view.)

Colorado Division of Water Resources, 2009, Diversion records, accessed January 30th, 2009, at http://www.water. state.co.us/pubs/datasearch.asp.

Colorado Water Quality Forum, 2008, Nutrient criteria, accessed April 7, 2010, at http://www.cwqf.org/.

Eagle County, 2009, Eagle County Colorado profile: accessed January 23, 2009, at http://www.eaglecounty.us/departmentcontent.aspx? $i d=3479 \&$ terms $=$ profile .

Eagle River Watershed Plan, 1996, A collaborative effort initiated by local governments: Eagle County Planning Commission, $101 \mathrm{p}$.

Fairchild, J.F., Allert, A.L., Poulton, B.C., and Grahm, R.V., 2002, A site-specific assessment of the risk of ammonia to endangered Colorado pikeminnow and razorback sucker populations in the upper Colorado River adjacent to the Atlas Mill tailings pile, Moab, Utah. Final report to the U.S. Fish and Wildlife Service, Division of Environmental 
Quality, Off-Refuge Contaminant Assessment Program: U.S. Geological Survey, Columbia Environmental Research Center, Columbia, Mo. and U.S. Environmental Protection Agency., Region 8, Denver, Colo.

Fangmeier, D.D., Elliot, W.J., Workman, S.R., Huffman, R.L., and Schwab, G.O., 2006, Soil and water conservation engineering, 5th ed.: Clifton Park, N.Y., Thompson Delmar Learning, $502 \mathrm{p}$.

Freeze, R.A., and Cherry, J.A., 1979, Groundwater: Englewood Cliffs, N.J., Prentice-Hall, Inc., 604 p.

Helsel, D.R., 2005, Non-detects and data analysis-Statistics for censored environmental data: New Jersey, John Wiley and Sons, Inc., 268 p.

Helsel, D.R., and Hirsch, R.M., 1992, Statistical methods in water resources: New York, Elsevier Science Publishers, $529 \mathrm{p}$.

Hem, J.D., 1989, Study and interpretation of the chemical characteristics of natural water (3rd ed.): U.S. Geological Survey Water-Supply Paper 2254, 263 p.

Julien, P.Y., 2010, Erosion and sedimentation: second edition, Cambridge, United Kingdom, The Cambridge University Press, 371 p.

Knighton, David, 1998, Fluvial forms and processes: a new perspective: London, Great Britian, Hodder education, part of Hachette Livre UK, 383 p.

Lanfear, K.J., and Alexander, R., 1990, Methodology to derive water-quality trends for use by the National Water Summary Program of the U.S. Geological Survey: U.S. Geological Survey Open-File Report 90-359, 10 p.

Mueller, D.K., Hamilton, P.A., Helsel, D.R., Hitt, K.J., and Ruddy, B.C., 1995, Nutrients in ground water and surface water of the United States-An analysis of data through 1992: U.S. Geological Survey Water-Resources Investigations Report 95-4031, 74 p.

National Park Service, 1998, Baseline water quality data inventory and analysis: Curecanti Recreation Area: Water Resources Division Technical Report NPS/NRWRD/ NRTR-98/181, 1,087 p.

Ries, K.G., III, Guthrie, J.G., Rea, A.H., Steeves, P.A., Stewart, D.W., 2008, StreamStats: A water resources web application: U.S. Geological Survey Fact Sheet 2008-3067, 6 p.

Rupert, M.G., and Plummer, L.N., 2009, Groundwater quality, age, and probability of contamination, Eagle River Watershed valley-fill aquifer, north-central Colorado, 2006-2007: U.S. Geological Survey Scientific Investigations Report 2009-5082, 59 p.

Santore, R.C., Di Toro, D.M., Paquin, P.R., Allen, H.E., and Meyer, J.S., 2001, Biotic ligand model of the acute toxicity of metals: Environmental Toxicology and Chemistry, v. 20, no. 10 , p. 2397-2402.

Schemel, L.E., Kimball, B.A., and Bencala, K.E., 1999, Colloid formation and the transport of aluminum and iron in the Animas River near Silverton, Colorado, in Morganwalp, D.W., and Buxton, H.T., eds., U.S. Geological Survey Toxic Substances Hydrology Program-Proceedings of the technical meeting, Charleston, South Carolina, March 8-12, 1999U.S. Geological Survey Water-Resources Investigations Report 99-4018A, p. 59-62.

Stumm, W., and Morgan, J.J., 1996, Aquatic chemistrychemical equilibria and rates in natural waters: New York, Wiley Interscience, 1,022 p.

Seaber, P.R., Kapinos, F.P., and Knapp, G.L., 1987, Hydrologic unit maps: U.S. Geological Survey Water Supply Paper 2294, 63 p.

Tweto, Ogden, comp., 1979, Geologic map of Colorado: U.S. Geologic Survey State Geologic Map, scale 1:500,000 (reprinted).

U.S. Census Bureau, 2009, State and county QuickFacts: accessed July 14, 2009, at http://quickfacts.census.gov/qfd/ states $/ 08 / 08037 . h t m l$.

U.S. Environmental Protection Agency, 1986, Quality criteria for water, 1986: U.S. Environmental Protection Agency Report 440/5-86-001, variously paginated.

U.S. Environmental Protection Agency, 2000, Nutrient criteria technical guidance manual: river and streams, 2000: U.S. Environmental Protection Agency Report 822B-00-002.

U.S. Geological Survey, 2008, Streamstats national dataCollection station information: accessed February 24, 2009, at http://streamstats.usgs.gov/gages/viewer.htm.

U.S. Geological Survey, 2010, The National map seamless server, National Agriculture Imagery Program (NAIP) 1-meter orthoimagery for zone 12 quarter quadrangle: U.S. Department of Agriculture, accessed October 15, 2008, at http://seamless.usgs.gov/.

U.S. Geological Survey, 2007, Water-resources data for the United States water year 2007: U.S. Geological Survey Water-Data Report WDR-US-2007, accessed October 11, 2008, at http://wdr.water.usgs.gov/wy2007.

Western Regional Climate Center, 2009, Local climatological data for Eagle and Climax, Colorado: accessed January 23, 2009, at http://www.wrcc.dri.edu/cgi-bin/ cliMAIN.pl?co2454.

Wilson, A.B., and Sims, P.K., 2003, Colorado mineral belt revisited-An analysis of new data: U.S. Geological Survey Open-File Report 2003-46, 7 p. 
Wynn, K.H., Bauch, N.J., and Driver, N.E., 2001, Gore Creek watershed, Colorado-Assessment of historical and current water quantity, water quality, and aquatic ecology, 1968-98: U.S. Geological Survey Water-Resources Investigations Report 99-4270, 72 p.

Zuellig, R.E., Bruce, J.F., Healy, B.D., Williams, C.A., 2010, Macroinvertebrate-based assessment of biological condition at selected sites in the Eagle River watershed, Colorado, 2000-07: U.S. Geological Survey Scientific Investigations Report 2010-5148, 19 p.

Publishing support provided by:

Denver Publishing Service Center

For more information concerning this publication, contact:

Director, USGS Colorado Water Science Center

Box 25046, Mail Stop 415

Denver, CO 80225

(303) 236-4882

Or visit the Colorado Water Science Center Web site at:

http://co.water.usgs.gov/ 


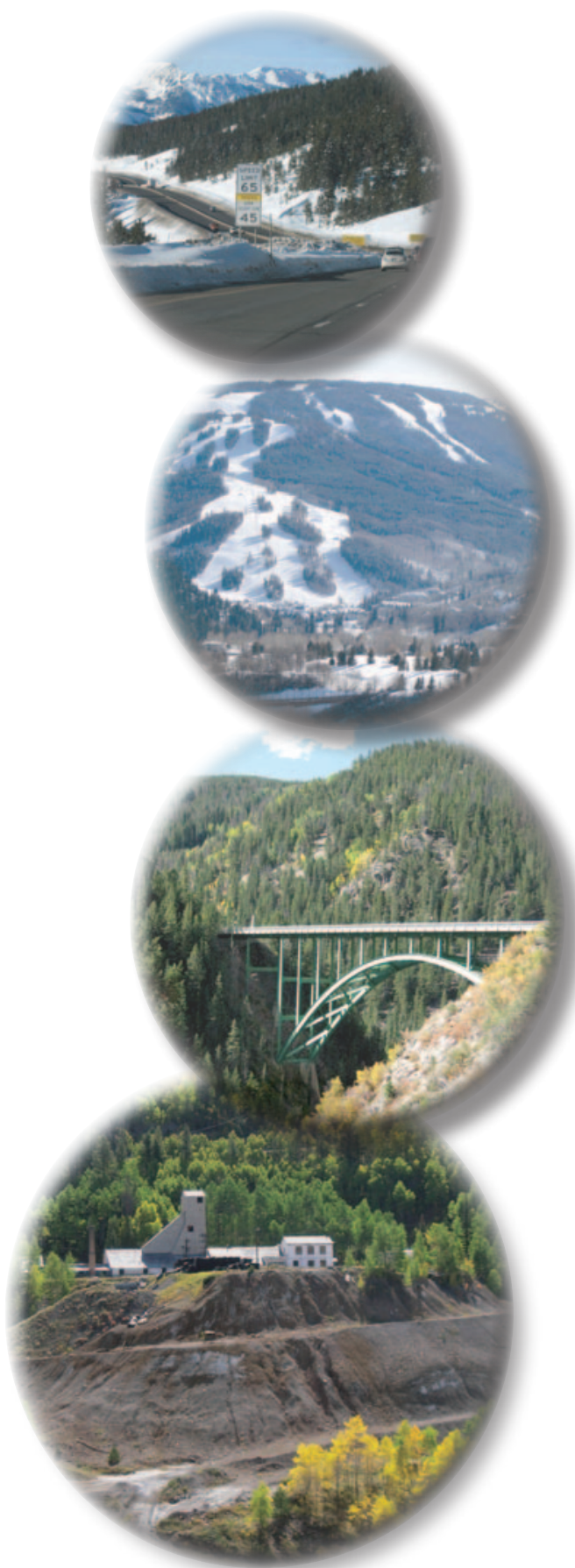

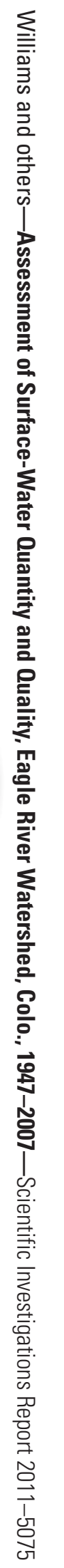

